Programs of the

Manufacturing

Engineering

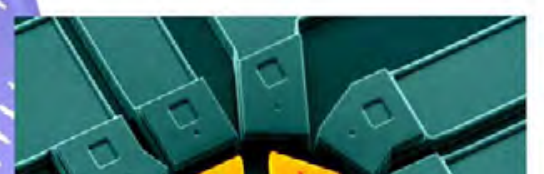

Laboratory
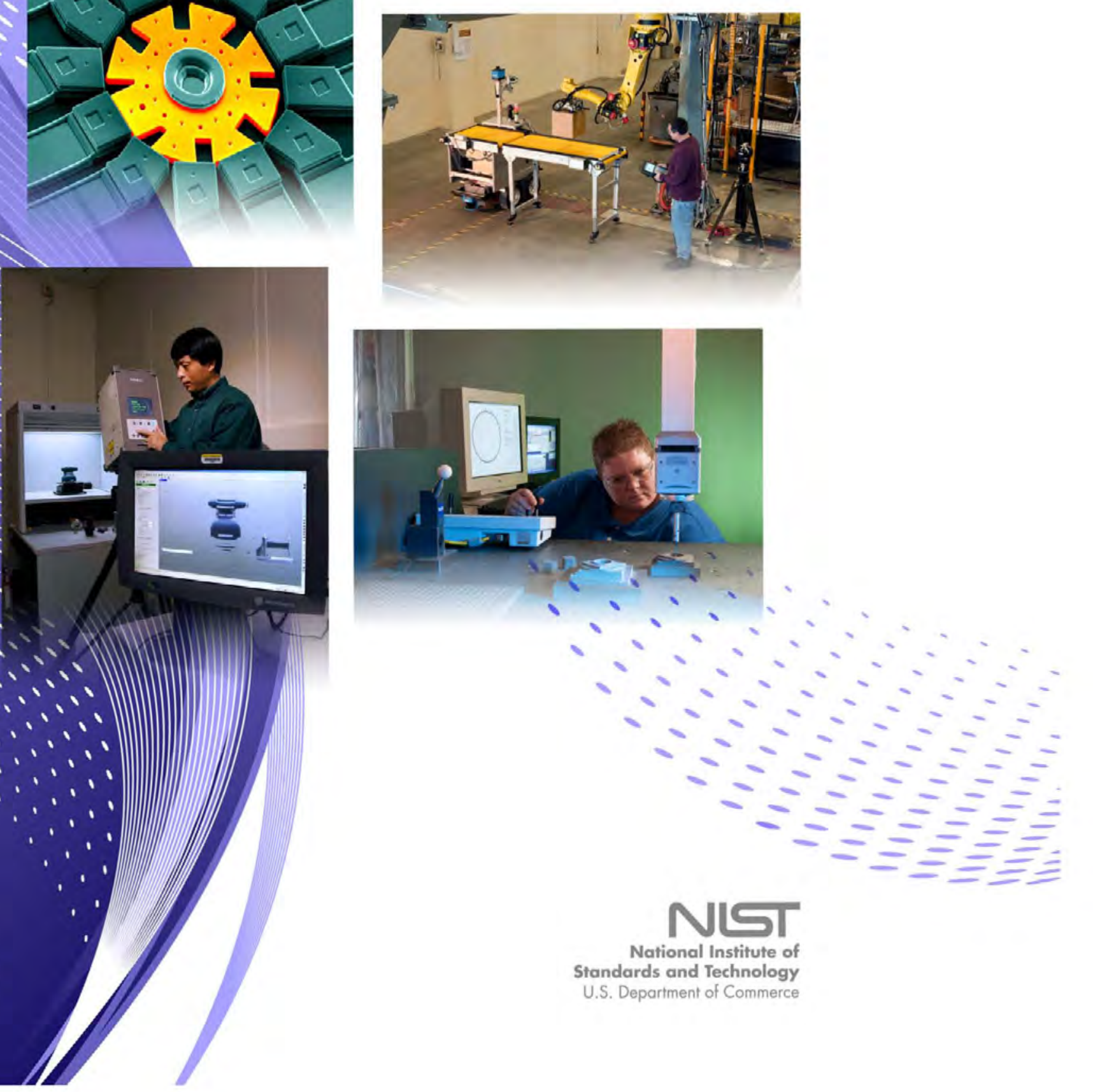
Standards and Technology 


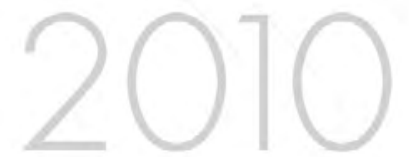

\title{
Programs of the
}

\section{Manufacturing}

Engineering

\section{Laboratory}

\author{
March 2010
}




\section{Abstract}

The National Institute of Standards and Technology's Manufacturing Engineering Laboratory (MEL) promotes innovation and the competitiveness of U.S. manufacturing through measurement science, measurement services, and critical technical contributions to standards. This report contains highlights of MEL research programs and accomplishments from April 2008 through March 2010.

\section{Keywords}

Manufacturing, manufacturing engineering, technology, measurements, metrology, standards

\section{Disclaimer}

Certain commercial equipment, instruments, or materials are identified in this report to specify the experimental procedure adequately. Such identification is not intended to imply recommendations or endorsement by the National Institute of Standards and Technology, nor is it intended to imply that the materials or equipment identified are necessarily the best available for the purpose.

\section{For More Information}

Manufacturing Engineering Laboratory

Stop 8200

Gaithersburg, MD 20899-8200

Phone 301-975-3400

www.nist.gov/mel 


\section{Table of Contents}

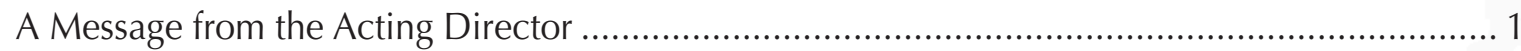

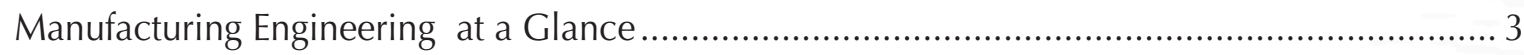

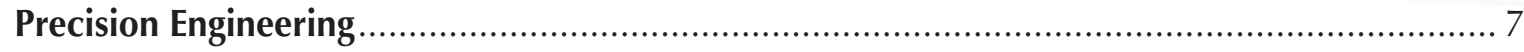

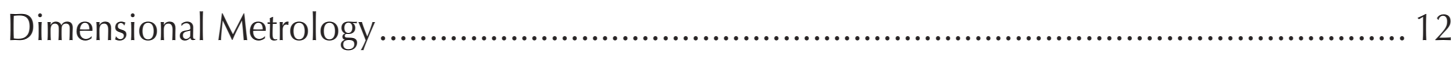

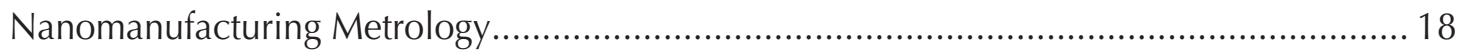

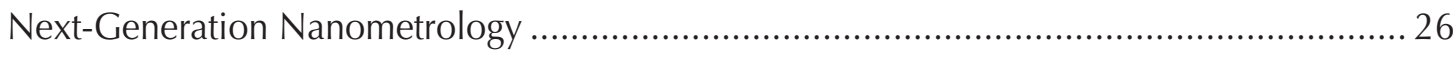

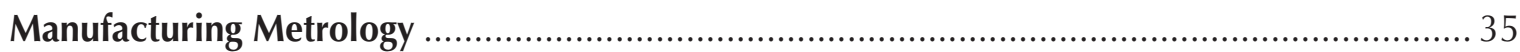

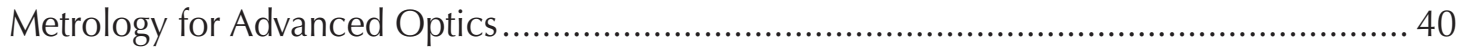

Measurements and Standards for Science-Based Manufacturing ................................... 47

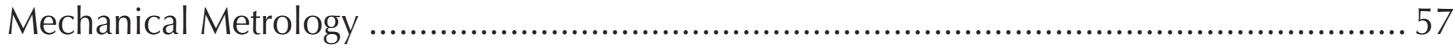

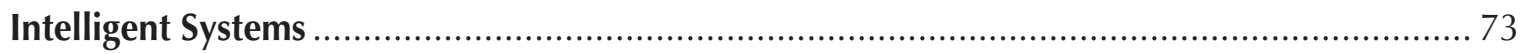

Intelligent Manufacturing Industrial Control Systems \& Network Standards ....................... 76

Intelligent Manufacturing Robotics and Automation Interoperability Standards................... 82

Measurement Science for Intelligent Manufacturing Robotics and Automation ................... 89

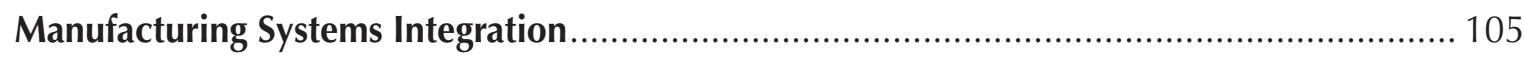

Sustainable and Lifecycle Information-based Manufacturing ....................................... 108

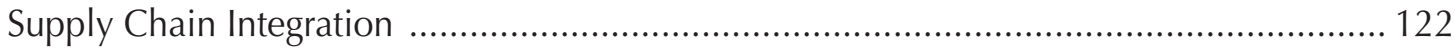

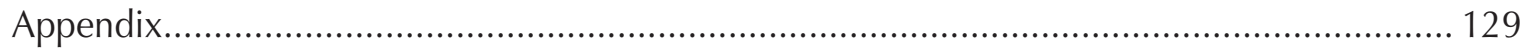

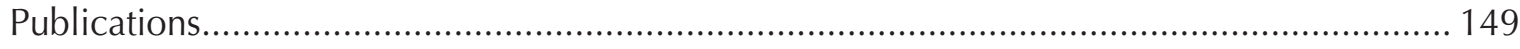




\section{A Message from the Acting Director}

T he Manufacturing Engineering Laboratory (MEL) at the National Institute of Standards and Technology (NIST) is pleased to present summaries of its technical work covering March 2008 through February 2010. MEL's mission is to promote innovation and the competitiveness of U.S. manufacturing through measurement science, measurement services, and critical technical contributions to standards. We do this by performing research and development, developing and providing needed measurement services, and providing technical contributions and leadership in standards activities. Our long-term goal is best summed up by the Laboratory's core purpose - to promote a healthy U.S. manufacturing economy by solving tomorrow's measurement and standards problems today.

The economic difficulties of the past two years have drawn attention to the central role that manufacturing plays in the US economy. The President's Framework for Revitalizing American Manufacturing recognizes that "America's manufacturers are at the heart of our country's economy, providing good-paying jobs for millions of American families." With a value-added contribution of $\$ 1.6$ trillion, U.S. manufacturing - our customer base - directly accounts for approximately $11.5 \%$ of the U.S. gross domestic product. Dollar for dollar, manufacturing has the highest-leverage economic impact of all of the economic sectors. For every dollar of economic output produced by manufacturing, $\$ 1.40$ of additional output is stimulated in the rest of the economy. As such, the ability of domestic manufacturers to innovate and compete is vital to all the other sectors of the economy.

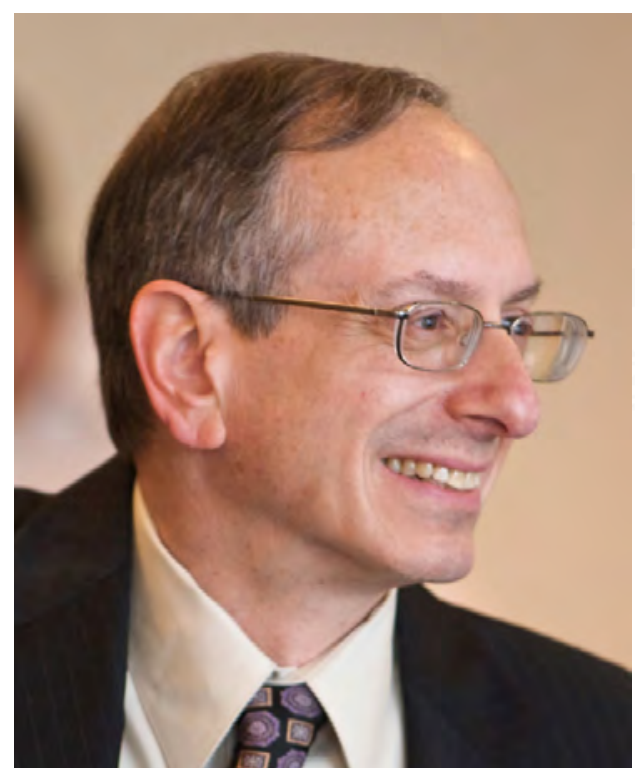

MEL brings to bear considerable resources in support of U.S. manufacturing, including dedicated staff and associates, and numerous unique facilities. MEL staff competencies span a broad range of technical disciplines, reflecting the multidisciplinary nature of manufacturing engineering, and have the manufacturing domain expertise needed to understand and address manufacturers' measurement and standards challenges. The pages that follow provide an opportunity to learn more about MEL's programs and capabilities, and how they are applied to solve critical industry problems.

MEL serves the manufacturing sector of the U.S. economy in a broad sense, working with partners from industry as well as other government agencies and academia to develop the measurement tools and infrastructure that enable new products, higher productivity, and improved processes. Our customers span the full range from established to emerging-technology industries — including automotive, aerospace, construction and agricultural equipment, medical devices, microelectronics, optics, telecommunications, and nanotechnology. MEL also provides mechanical design and fabrication services to other NIST operating units through its stewardship of the Fabrication Technology Division. 
Nearly all of the nation's manufacturers rely on MEL in some way, and we design our programs, in consonance with our mission, to respond to manufacturing's most critical current and future needs. Working collaboratively with our external partners, MEL staff members solve measurement and standards problems that allow our customers to overcome barriers to product and process innovation, to seamlessly and accurately sha manufacturing information, and to take full advanta of the latest technologies essential to their competiti ness and mission success. In the last two years, we have placed increasing emphasis on research in are increasing strategic and tactical interest to our cons..... ency: sustainable manufacturing, next generation robotics, in-process dimensional metrology, and additive manufacturing. A key strategy in many of our activities is to use performance metrics and performance-based standards as a means to catalyze innovation. Our customers also depend on MEL calibration services to meet their most challenging mechanical and dimensional measurement needs for research, development — and the requirements of the modern extended enterprise in commerce, and international trade.
This document will give you a sense of the challenges faced by U.S. manufacturers in areas related to the MEL mission, and how we are addressing them. As you review our portfolio of technical activities, we invite your comments and questions, we welcome opportunities to collaborate, and we thank you for your interest in our work.

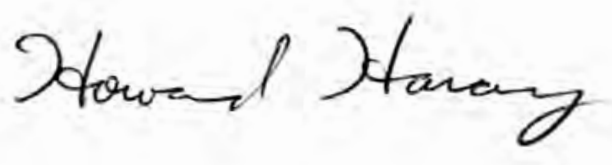

Manuıdııי's ‘.'o..... v

National Institute of Standards and Technology

Gaithersburg, Maryland 20899

Howard.Harary@NIST.gov

3019753401 


\section{Manufacturing

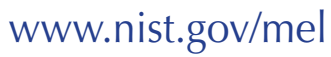 \\ Engineering at a Glance}

\section{MEL Mission}

MEL promotes innovation and the competitiveness of U.S. manufacturing through measurement science, measurement services, and critical technical contributions to standards

\section{MEL Vision}

MEL will be the world leader in creating critical measurement solutions and promoting technically rigorous, equitable standards to stimulate innovation and foster U.S. manufacturing competitiveness

\section{MEL Core Purpose}

To promote a healthy U.S. manufacturing economy by solving tomorrow's measurement and standards problems today

\section{MEL Divisions}

\section{Precision Engineering}

Provides the foundation for dimensional measurements over 12 orders of magnitude

\section{Manufacturing Metrology}

Fulfills mechanical metrology and advanced manufacturing technology measurements and standards needs

\section{Intelligent Systems}

Provides the measurement and standards infrastructure needed for the application of intelligent systems

\section{Manufacturing Systems Integration}

Develops and applies measurements and standards that advance the use of information-based manufacturing technologies

\section{Fabrication Technology}

Provides world-class instrument and specialized fabrication support for NIST researchers

\section{Measurement Service Areas}

- Length

- Diameter and Roundness

- Complex Dimensional Standards

- Optical Reference Plane Standards

- Angular Measurements

- Surface Texture

- Laser Frequency/Wavelength and Ranging

- Mass Standards

- Force

- Vibration

- Acoustics

\section{MEL Resources}

\section{Staff}

- 171 Full-time permanent

- 99 Guest Researchers

- 2 NRC postdoctoral researchers

\section{Funding}

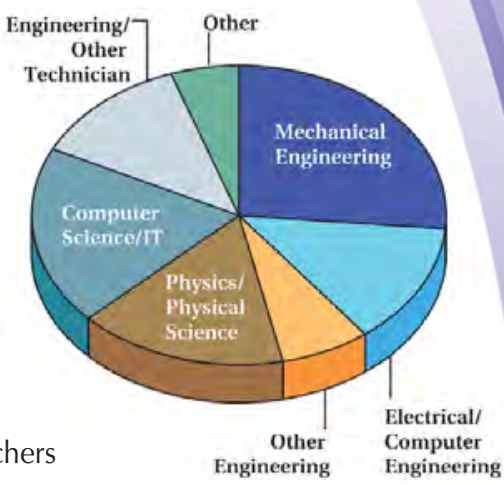

$\$ 48.7 \mathrm{M}$ annual budget

(FY2010 estimated, excludes Shops)

- \$35.5 M NIST Appropriations

- $\$ 7.1 \mathrm{M}$ Other Agency/External R\&D

- \$4.8 M Calibration service fees/Reimbursable

\section{Facilities and Testbeds}

- M-48 Coordinate Measuring Machine Laboratory

- 60-meter Laser \& Artifact Calibration Range

- Next-Generation Nanometrology Laboratories

- Mass Metrology and Standards Facility

- Force Metrology Laboratories

- Small Force Metrology Laboratory

- Smart Machining And Machine Tool Interoperability Testbed

- Dimensional Metrology Interoperability Testbed

- Industrial Control Networking and Cybersecurity Testbed

- Industrial Robotics Measurement Science Testbed

- Advanced Manufacturing Systems And Networking Testbed (AMSANT) and Simulation Laboratories 


\section{MEL American Recovery and Reinvestment Act (ARRA) Equipment and Facility Investments}

$T^{T}$ he American Recovery and Reinvestment Act (ARRA) provides funding to MEL for high value research, measurement, and production equipment, and funding for a Robot Test Facility, to better meet our customers' current and future needs. The table below presents a summary of MEL equipment to be procured with ARRA funds.

\begin{tabular}{|c|c|c|}
\hline Item & Division & Purpose \\
\hline $\begin{array}{l}\text { Critical Dimension } \\
\text { Atomic Force } \\
\text { Microscope (CD-AFM) }\end{array}$ & PED & $\begin{array}{l}\text { Provide reference linewidth measurements with } 1 \mathrm{~nm} \text { uncertainty. The instrument } \\
\text { will replace an earlier generation CD-AFM, with improved uncertainty to meet } \\
\text { increasingly demanding industry needs. }\end{array}$ \\
\hline $\begin{array}{l}\text { Atom Metrology } \\
\text { Scanning Electron } \\
\text { Microscope }\end{array}$ & PED & $\begin{array}{l}\text { Provide 3D traceable metrology in the } 10 \mathrm{~mm} \text { to } 10 \mathrm{~nm} \text { range for nano-, nano-bio- } \\
\text { and integrated circuit technology measurements and standards calibrations. }\end{array}$ \\
\hline $\begin{array}{l}\text { Line Scale Interfer- } \\
\text { ometer }\end{array}$ & PED & $\begin{array}{l}\text { Ensure continued and improved calibration of fundamental macro-scale artifacts } \\
\text { that are critical to manufacturing quality control, such as graduated length scales } \\
\text { and lithographic grids. The nearly } 50 \text { year-old instrument used to support these } \\
\text { high-impact calibrations of industry fundamental length standards has exceeded its } \\
\text { life expectancy, has become unsupportable, and likely will fail completely in the } \\
\text { near future. }\end{array}$ \\
\hline $\begin{array}{l}\text { Calibrated Atomic } \\
\text { Force Microscope }\end{array}$ & PED & $\begin{array}{l}\text { Provide fundamental nanoscale length metrology, especially, pitch and step height, } \\
\text { for customers in the semiconductor manufacturing, optics and photonics, data stor- } \\
\text { age, and biomedical industries among others. Capabilities include } 300 \mathrm{~mm} \text { wafer } \\
\text { capacity (upgradeable), tip characterization, image stitching, and dimensional } \\
\text { aspects of material characterization, required for both our external and NIST-inter- } \\
\text { nal customers. }\end{array}$ \\
\hline Large Mass Standards & MMD & $\begin{array}{l}\text { Enhance reliability of the realization/dissemination of the SI unit of Mass at the } \\
\text { large scales }(11.12 \mathrm{kN}[2500 \mathrm{lb}], 44.48 \mathrm{kN}[10,000 \mathrm{lb}] \text { and } 88.97 \mathrm{kN}[20,000 \\
\mathrm{lb}]) \text { ). New stainless steel standards will significantly reduce the current Type B } \\
\text { uncertainty measurements related to material properties so that NIST large mass } \\
\text { calibration services continue to meet performance demands for the U.S. military, } \\
\text { nuclear power industry, aerospace companies, and the State Weights and Measures } \\
\text { Laboratories that disseminate the unit of mass throughout the country. }\end{array}$ \\
\hline $\begin{array}{l}\text { Metal-based Addi- } \\
\text { tive Manufacturing } \\
\text { System }\end{array}$ & MMD/FTD & $\begin{array}{l}\text { Serve as a testbed for research on emerging metal-based additive manufacturing } \\
\text { processes. The research will address measurement and standards barriers to the } \\
\text { adoption of these processes by U.S. manufacturers to improve innovation and com- } \\
\text { petitiveness, and the development and application of metrology tools for science- } \\
\text { based process improvement. }\end{array}$ \\
\hline $\begin{array}{l}\text { Micro-wire and EDM } \\
\text { Machines }\end{array}$ & FTD & $\begin{array}{l}\text { Ultra-precision CNC Wire EDM Machine to fabricate parts for Nanomanufacturing } \\
\text { and Nanometrology programs ( } 0.03-0.01 \mathrm{~mm} \text { feature sizes) }\end{array}$ \\
\hline $\begin{array}{l}\text { CNC Vertical and } \\
\text { Horizontal Milling } \\
\text { Machines }\end{array}$ & FTD & CNC Mills to replace aging manual mills in Contact Shops and Main Shop \\
\hline $\begin{array}{l}\text { CNC Long-bed and } \\
\text { Tool Room Lathes }\end{array}$ & FTD & $\begin{array}{l}\text { Large Long-bed CNC Lathe to replace aging manual } 15^{\prime \prime}(0.381 \mathrm{~m}) \text { chuck lathe in } \\
\text { Main Shop; Tool Room Lathes } 10^{\prime \prime}(0.254 \mathrm{~m}) \text { dia capacity to replace aging manual } \\
\text { lathes in Shop }\end{array}$ \\
\hline $\begin{array}{l}5 \text {-axis CNC } \\
\text { Machining Center }\end{array}$ & FTD & Ultra-precision 5-axis CNC Machining Center to fabricate complex contoured parts \\
\hline
\end{tabular}




\section{T}

he new NIST Robot Test Facility will be a unique national resource for testing and improving the performance of robots used in search-and-rescue, law enforcement, and homeland and national defense missions. The facility is intended to replace existing test facilities that are currently housed on a decommissioned Nike defensive missile site near the main NIST campus. The $650 \mathrm{~m}^{2}$ to $762 \mathrm{~m}^{2}$ facility is to include areas for robot test methods and artifacts, development and fabrication, and briefings and test administration. The supported projects facilitate manufacturing and deployment of advanced robotic capabilities through standard performance test methods, measurement services, and safety and training guidelines. These efforts are largely funded by other federal agency sponsors, and they have important synergies with MEL's manufacturing robotics work.

\section{NIST Robot Test Facility Elevations and Floor Plan}
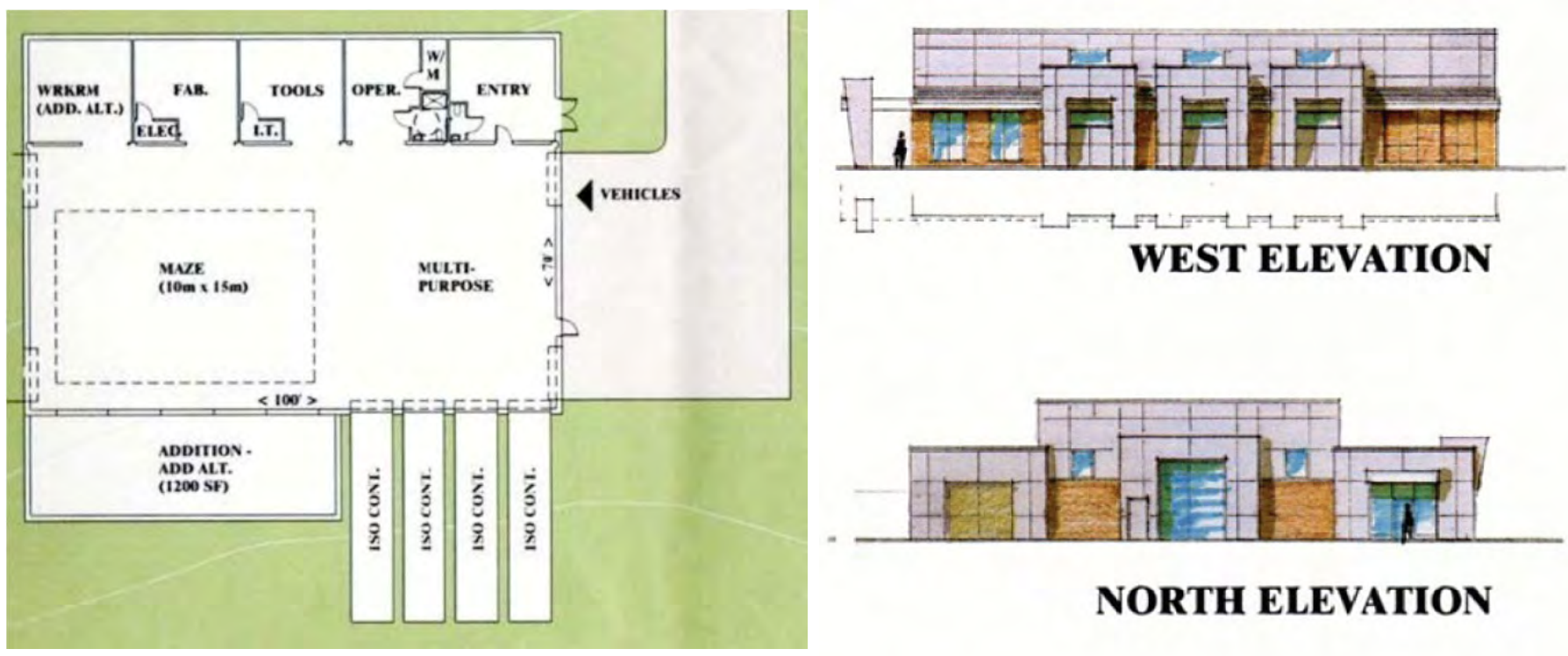

WEST ELEVATION

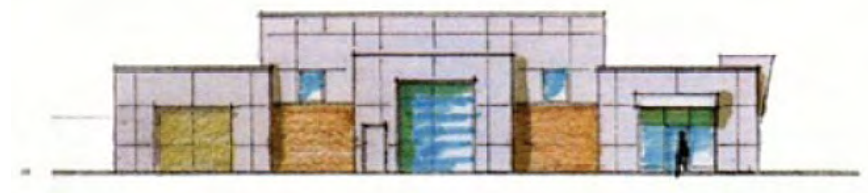

NORTH ELEVATION

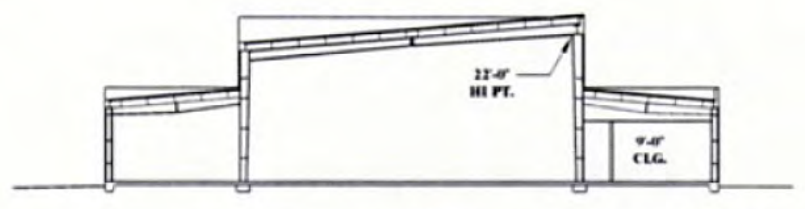

BUILDING SECTION 




\section{Mission}

he mission of the Precision Engineering Division is to provide the foundation of dimensional measurement that meets the needs of the U.S. industrial and scientific communities. The division attains that goal by:

- Conducting research in dimensional measurements

- Developing new measurement methods

- Providing measurement services

- Developing National and International artifact and documentary standards

- Disseminating the resulting technology and lengthbased standards

It is also within the mission of the Precision Engineering Division to provide dimensional metrology assistance to other federal government agencies to address problems and needs that leverage NIST expertise, facilities, and capabilities.

\section{Overview}

D ED is committed to developing the metrology needed to support U. S. manufacturing and has been instrumental in the development of instrumentation, metrology and standards especially for the semiconductor, automotive and aerospace industries. PED is also a primary partner in the development of the needed metrology for the Department of Defense and many other agencies. PED has also been a strong contributor to the National Nanotechnology Initiative (NNI). The continual push of the semiconductor industry to smaller and smaller gate structures has also pushed PED to ever improving nanoscale measurements and standards and a drive to encourage instrument manufacturers for higher performance. This has strategically positioned PED for leadership in the development of accurate metrology for nanotechnology and nanomanufacturing. Thus, for over a decade, the Manufacturing Engineering Laboratory and the Precision Engineering Division have been deeply involved in the development of metrology for the nascent nanomanufacturing industry (Lyons and Postek, 2009). ${ }^{1}$

\footnotetext{
1 Lyons, K., and Postek, M. T. 2009. Historic commitment of the NIST Manufacturing Engineering Laboratory to nanomanufacturing and nanometrology. SPIE Proceedings. Proc. SPIE 7405 740503-1 - 740503-18.
}

The Precision Engineering Division (821.00) is broken into four managerial units or groups and these are:

- Large-Scale Coordinate Metrology Group (821.11) Supports the Division's mission with a concentration on measurements of one meter or larger. For example, measurements are made by coordinate measuring machines and frameless coordinate length-metrology systems. These measurements involve mechanicalprobe, laser-ranging, theodolite, and related interferometric systems.

- Engineering Metrology Group (821.12) Supports the Division's mission with a concentration on measurements of one millimeter to one meter. These measurements are often of complex shape, such as turbine blades, threaded fasteners, and gears. Measurements are made by general and special-purpose feature and coordinate measuring systems.

- Surface and Microform Metrology Group (821.13) Supports the Division's mission with a concentration on measurements of one micrometer to one millimeter where surface roughness and microform are critical. Measurements are made by tunneling microscopes, mechanical profilometry, phase-measuring interferometry and related techniques.

- Nanoscale Metrology Group (821.14) Supports the Division's mission with a concentration on dimensional measurements of one nanometer to one micrometer. Measurements are made by tunneling, atomic-force, electron, ion, and visible- and ultraviolet-light microscopies. The group places special emphasis on satisfying the advanced needs of U.S. microelectronic manufacturing industries.

MEL/PED Programs. Noting that the talents of workers within one group are valuable in cross divisional and even cross laboratory collaborations, the Precision Engineering Division has developed three programs to best utilize the talents of its personnel and thus meet its mission with maximum effectiveness. These three programs are:

- Dimensional Metrology

- Nanomanufacturing Metrology

- Next-Generation Nanometrology 
Core competencies and Industry Drivers. The core competencies of the PED Programs reside in measurement science and rigorous traceability in the mechanical, dimensional, and nanomanufacturing domains. The ability to understand and develop a measure of measurement uncertainty for the development of accurate dimensional metrology is a core competency unparalleled in the world.

The work of the three programs is strongly influenced by industrial drivers that reflect important trends in manufacturing, especially:

- Increased Pace of Technological Change.

- Rapid innovation in many emerging areas, especially nanotechnology

- Need for improved measurement accuracy and precision

- Requirement for new and more complex measurements

- Globalization

- Flat-world ${ }^{2}$ supply chains require measurement traceability to the International System of Units (SI, from Systeme International d'Unités)

- Greater regulatory requirements

- Harmonization of international standards that reflect and support U.S. needs

The implications of these drivers for PED include:

- Technology Change, generating demand for new metrologies

- Globalization, leading to increased dependence on traceable measurements and international documentary standards

- Globalization, requiring integration and harmonization between formerly separate industry sectors, each with entrenched practices and terminologies (this is especially true for nanotechnology)

2 "The World is Flat" by Thomas L. Friedman. columnist at The New York Times

\section{Staffing Challenges}

O ne of the main challenges confronting PED continues to be a "graying" of the highly trained and specialized metrology workforce. This, coupled with reduced budget, limits the ability to train replacements in place. As a result, the contractor labor pool has grown while the Federal staff-to-contractor ratio continues to fall. Additionally, trained contractors can readily leave NIST, taking their training and experience to outside companies, further exacerbating the problem. In addition, contracting a workforce is only a partial solution since intellectual property (IP) issues and government regulations can restrain a contractor from working on particular projects.

PED is also facing a steadily decreasing technician-to-professional ratio, again for budgetary reasons. The complexity of the highly specialized metrology instrumentation within PED (helium ion microscope, scanning electron microscopes, etc.) requires dedicated instrument technical professionals to operate and maintain the instrumentation at the high levels demanded by the metrologies.

\section{Facilities and Equipment}

PED was fortunate in obtaining about $\$ 5 \mathrm{M}$ of the American Reinvestment and Recovery Act (AARA) funds provided to NIST for the procurement of new stateof-the-art equipment. These funds are being used to purchase the following equipment:

- New Linescale Interferometer (LSI) -The current LSI has proven world class performance in recent international intercomparisons, but is a 50-year-old instrument with fragile and fail-susceptible sub-systems that is in urgent need of replacement. The new LSI will be operated in parallel with the existing LSI for a few years to ensure continuity in the measurement control history.

- New Calibrated Atomic Force Microscope (AFM) The new Traceable AFM (T-AFM) will provide fun- 
damental traceable nanoscale length metrology for customers in industries such as semiconductor manufacturing, optics and photonics, data storage, and biomedical among others. It will be used to measure height, pitch, surface roughness, line width roughness (top down), and other nanoscale features found on technologically relevant surfaces. The instrument will be traceable to the SI meter in all three axes.

- New Critical-Dimension (CD) AFM - This new instrument, will act as a reference for a wide variety of measurements in semiconductor dimensional metrology, and will be calibrated to have traceability to the definition of the meter through the use of atomic lattice based techniques.

- New Atomic Resolution Particle Beam Instrument The state-of-the-art instrument, combined with the sophisticated imaging methodology and metrology expertise within PED, will give us unique capabilities for characterizing and measuring nanometer-sized objects and features, unequaled anywhere in the world, and very necessary for the advancement of nanoscience and nanotechnology.

These instruments remain in the procurement cycle and once contracts are released, renovation of appropriate space within the NIST Advanced Measurement Laboratory (AML) will begin.

\section{Current Equipment and Facilities include:}

More extensive equipment descriptions are included in the PED materials provided.

\section{Building 220}

- World class 60 meter laser \& artifact calibration range.

- Large scale coordinate metrology laboratory.

- LineScale Interferometer.

- Optical Tweezers

\section{Building 219}

- World's most accurate coordinate measuring machine

- World-class roundness measuring machine

- Laser frequency / wavelength laboratory

- Molecular Measuring Machine

- Five chamber atomic resolution Ultra-High Vacuum Scanning Tunneling Microscope (UHV STM) with picometer resolution interferometry

- Highest resolution $193 \mathrm{~nm}$ wavelength fully custom scatterfield optical microscope

- High resolution Variable Pressure scanning electron microscope (SEM) Reference Measurement System

- World's most accurate Microform Calibration System for Rockwell Hardness Standardization

- A Unique NIST 2D and 3D Ballistics Signature Measurement and Correlation System

\section{Building 218}

- Calibrated Atomic Force Microscope (AFM). Custombuilt AFM with active interferometer-based metrology on $X$ and $Y$ axes, and in situ interferometer calibrated capacitance gage on $\mathrm{Z}$ axis

\section{Building 217}

- World's First Commercial Helium Ion Microscope

- High resolution Cold Field Emission scanning transmission electron microscope (STEM) / SEM

- High-resolution dual beam Focused-ion Beam (FIB) and SEM with laser interferometer stage

- World's first scatterfield optical microscope with demonstrated sub-nanometer resolution

- Optical overlay microscope Instrument

- Ultraviolet (UV) Transmission Microscope 


\section{Facilities and Equipment Challenges}

D ED is continually challenged by its customers to develop metrology solutions that go beyond current capabilities. One of the most demanding customers has been the semiconductor industry, which has been pushing the metrology envelope for many years via the International Technology Roadmap for Semiconductors (ITRS). Meeting the semiconductor industry's needs gave PED a head start when the emphasis on nanotechnology became apparent because PED had already been developing methodologies and performing measurements at the sub-100 nanometer scale. Hence, basic infrastructural technologies were already in place to support the metrology. PED's work has been facilitated by the new laboratories now residing in the Advanced Measurement Laboratory and the procurement and future delivery of the AARA equipment.

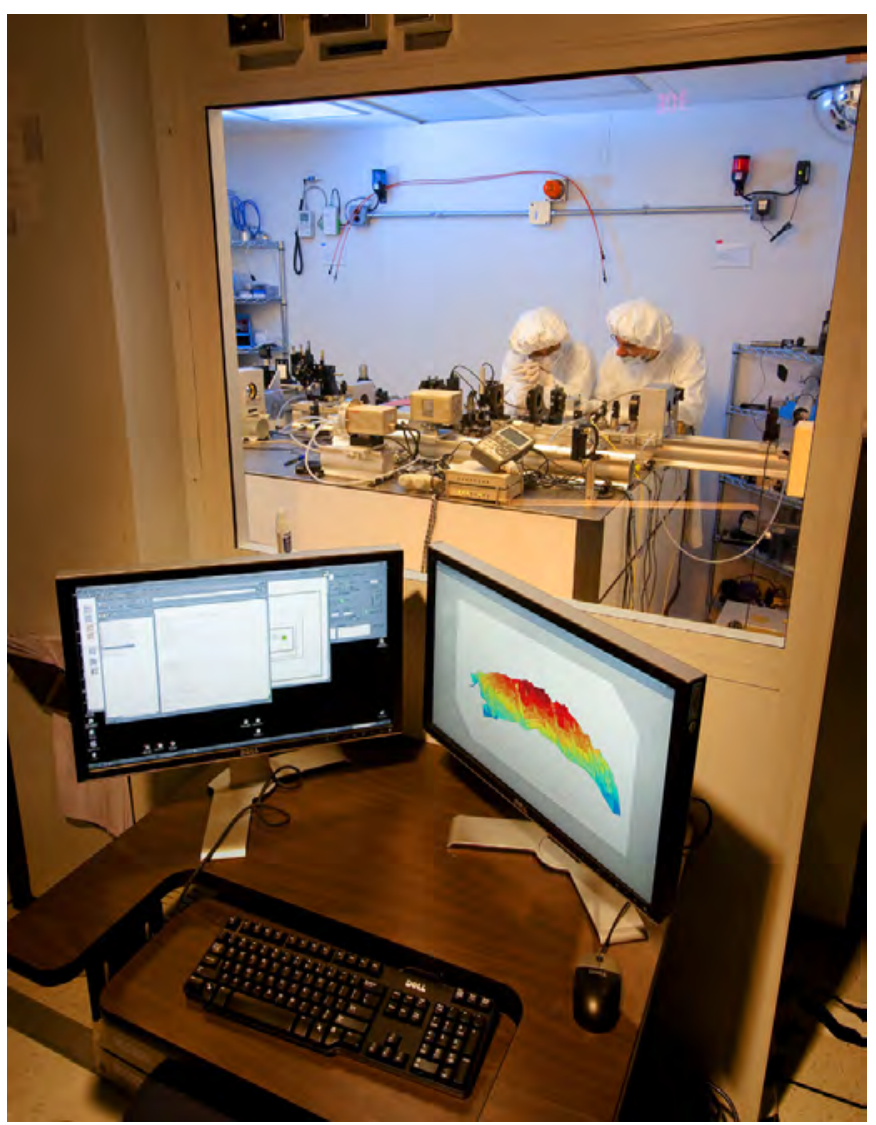

193 nm Scatterfield microscope
PED Advanced Measurement Laboratory (AML) Facilities. Over 20 years ago, PED proposed to NIST management a vision for an advanced measurement laboratory with stringent environmental controls to meet the future metrology needs of the United States. This facility has now been built and most of PED's metrology equipment has been installed there. Numerous metrology experiments have experienced a wide variety of improvements through this move. For example, in the new scanning electron microscope reference measurement instrument laboratory, site surveys by the instrument manufacturer prior to the microscope's installation found no measurable vibration. The instrument manufacturer's Applications Laboratory representative was quoted as saying that "there is no environmental excuse we can use in this laboratory to blame for poor performance of our instrument." In all cases, improved performance of the metrology instruments has been documented.

\section{Safety}

ince the last panel visit, NIST has undergone a massive safety campaign. Although safety in PED has always been excellent and no major incidents have occurred, PED management has undertaken hazard reviews of all of the laboratories and upgraded the safety procedures throughout. All of the managers within PED have been trained in Occupational Safety and Health Administration (OSHA) 6000 procedures, a Division Safety Officer has been identified and the staff has received safety training as needed for the type of work being done (i.e., laser safety, confined space training, crane safety, etc.) 


\section{PED Program}

\section{Dimensional Metrology}

Addressing selected needs in dimensional metrology over length scales ranging from micrometers to kilometers. This includes calibrations of measuring instruments such as laser interferometers and laser trackers, a wide array of engineering gauges, standard reference materials, and specialized measurements, e.g., high accuracy coordinate measuring machines (CMMs). The program also provides expertise and representation of U. S. interests in national and international standards committees. The DMP frequently provides metrology expertise to other government agencies including The Department of Defense (DOD), Department of Energy (DOE), National Aeronautics and Space Administration (NASA), and National Institute of Justice (NIJ).

\section{Staff}

18.5 NIST staff

5.5 Guest researchers / Contractors

22.5 Total FTEs

\section{Challenge}

o promote innovation and enhance U.S. productivity

by optimizing MEL's dimensional metrology portfolio to take advantage of advances in information, computational, and optoelectronic measurement technology for high accuracy dimensional metrology.

\section{Technical Strategies}

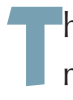
he DMP focuses on developing dimensional measurement infrastructure to support significant improvements in U.S. productivity. In particular, the Dimensional Metrology program (DMP) targets building new measurement capability for enabling technologies and high value products in which the U.S. has a significant industrial presence. Selected national and international standards, particularly in emerging technologies, are aggressively supported to promote international trade, foster innovation, and reduce manufacturing costs. The program also strives to increase the leverage of NIST's measurements through deep penetration of its measurements into the U.S. metrology chain.

\section{Selected Results and Impacts}

- Implemented a comprehensive quality system for all PED dimensional calibrations resulting in conformity with international accreditation practices.

- Developed the NIST micro-feature probe that provides the most accurate dimensional measurement of features in the $50 \mu \mathrm{m}$ to $100 \mu \mathrm{m}$ size range in the world.

- Harmonized the U.S. with the ISO standards for coordinate measuring machines by producing major revisions to both documents resulting in lower costs to U.S. manufacturing companies. 


\section{Publications}

Muralikrishnan B., Stone J., Stoup J., Area measurement of knife-edge and cylindrical apertures using ultra-low force contact fiber probe on a CMM, Metrologia 45 281-289, 2008

\section{Phillips, S.D., Krystek, M., Shakarji, C., and Summer-} hays, K., 2009. Three-Dimensional Imaging Metrology: Dimensional measurement traceability of 3D imaging data, Proceedings of the SPIE, Volume 7239, pp. 72390E-72390E-7.

J. Song, T. Vorburger, S. Ballou, T. Renegar, A. Zheng, and M. Ols, "Traceability for Ballistics Signature Measurements in Forensic Science," Journal of Measurement, DOI 10.1016/j.measurement.2009.

\section{Selected Staff Recognition}

\section{Board Memberships}

\section{Staff}

S.D. Phillips

S.D. Phillips

\section{Leadership}

\section{Staff}

S.D. Phillips and C. Shakarji

\section{Contact Information:}

Steven Phillips, Program Manager

301-975-3565

steven.phillips@nist.gov

\section{Board Memberships}

Board of the North American Coordinate Metrology

Association

Board for U.S. Standard Technical Advisory Group

\section{Excellence}

\section{Staff}

J. Stone

D. Sawyer and C. Shakarji

S.D. Phillips and W.T. Estler

S. D. Phillips, W. T. Estler, B. R. Borchardt,

C. Blackburn, D.S. Sawyer

\section{Leadership}

DOC Gold Medal, for leadership in

international standards

\section{Excellence}

DOC Silver Medal, for development of the NIST microfeature CMM probe

NIST Bronze Medal, for the design and fabrication of an operating room computer assisted orthopedic hip surgery artifact

2009. ASME's Certificate of Achievement" for outstanding national standards work

The NIST Judson C. French Award, for significant improvements in accuracy and extensions of range in the calibration of long length standards 


\section{Dimensional Metrology Projects}

\section{Ongoing Reimbursable Dimensional Calibrations and Quality System}

\section{Challenge/Problem Addressed}

.S. industries that involve dimensional metrology rely on NIST to provide state of the art high accuracy calibrations in order to maintain the measurement traceability and international competitiveness. To assure the international acceptance of NIST measurements, NIST has developed a Quality System based on the ISO 17025 Standard.

\section{Project Objective}

D rovide industry high accuracy dimensional measurements within an internationally accepted quality system. DMP measurements are targeted to provide measurement results that are either of very high value to our customers or high leverage into the industrial metrology community, i.e. a single calibration may propagate into hundreds or thousands of subsequent measurement results.

\section{Impact/Benefits}

MP delivered calibrations have very high market penetration, for example, master tapes calibrated at NIST control the millions of measuring tapes produced in the U.S. each year. Similarly, each NIST gauge block calibration explicitly controls - as cited by calibration report numbers - a thousand subsequent gauge block calibrations performed in industrial laboratories. The new, more flexible capability of the Moore M48 CMM has performed high value calibrations including cylinders for the Calculable Capacitor (NIST), alignment fixtures for the James Webb Telescope (NASA) and a triple retroreflector for the SIMlite Astrometric Observatory (NASA).

\section{Customers \& Collaborators}

DMP calibrations serve a wide range of industries including high end calibration laboratories, gauge and instrument manufactures, aerospace and defense contractors, and federal and state governments. In the period of FY2005 to FY2009 the DMP provided dimensional measurement services to 360 different industrial customers and 25 government entities. The DMP calibrates over 5,000 master gauges, instruments, and artifacts per year with gross revenues of approximately $\$ 0.8 \mathrm{M}$ per year.

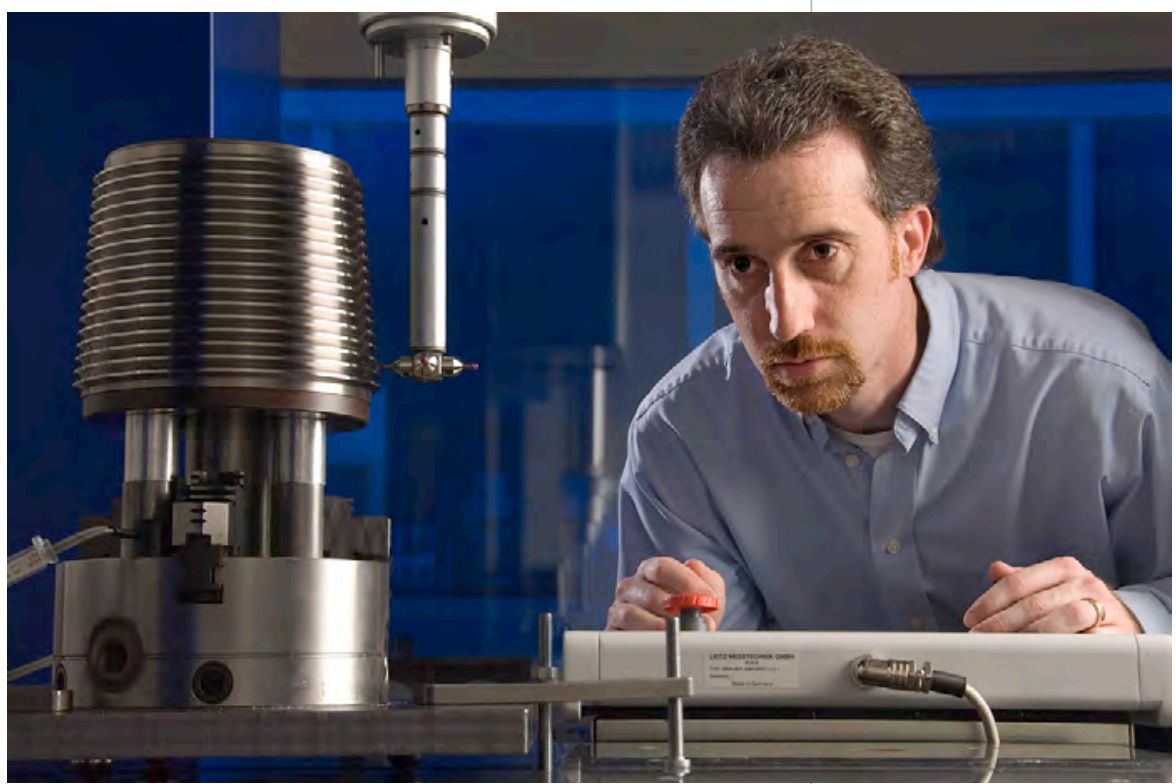

Measurement of API Rotary Master Gauge on CMM 


\section{Improvements in Calibrations and Uncertainty Evaluation}

\section{Challenge/Problem Addressed}

D ractical access to the International System of Units

(SI) unit of length for U.S. manufactures requires continuous upgrades to the DMP dimensional calibrations through reduced uncertainty, greater throughput, and new measurands.

\section{Project Objective}

D rovide industry with higher accuracy dimensional calibrations and more technologically relevant calibrations to enhance U.S. productivity and quality.

\section{Impact/Benefits}

New measurement capabilities and improved measurement uncertainties are listed below:

- Acquired UMAP multi-sensor Coordinate Measuring Machine (CMM) that extends range of small feature calibration below $50 \mu \mathrm{m}$ to provide microfeature calibration capability, 2009

- The second Moore M48 CMM was installed in the AML and is currently being error mapped. It will provide new video based calibration capability; 2010

\section{Customers}

Our industrial calibration customers and the general metrology community will benefit from improvements in theoretical infrastructure and the new measurement capabilities provided by extensions and improvements in the GUM and related uncertainty standards.

\section{Collaborators include}

Michigan Metrology, Detroit, Ml; Taylor Hobson, Chicago, IL;Veeco Metrology, Tucson, AZ; Zygo Corporation, Middlefield, CT; national and international standards laboratories and standard committees.

\section{Optical Comb and Refractometry}

\section{Challenge/Problem Addressed}

\section{A easurement accuracy originates with the realiza- $\checkmark$ tion of the SI meter and this project will allow} the PED to more accurately and compressively realize the meter. A better determination of the refractive index of air will enable more accurate laser wavelength (in air) measurements (or calculations), which is the basic limitation on the accuracy of interferometric length measurement. Additionally, this will also help determine the wavelength of lasers that do not operate at the usual red wavelength, satisfy the needs of multicolor interferometry, and enable new, novel measurement systems.

\section{Project Objective}

\begin{abstract}
dvancing the realization of the meter through the use of absolute refractometry to achieve below one part in $10^{8}$ accuracy. Realization of practical frequency standards from the comb are expected by 2011.
\end{abstract}

\section{Impact/Benefits}

$\mathrm{t}$ is anticipated that deployment of the refractometer into other PED calibration instruments, including the Moore M48 CMMs, will reduce the measurement uncertainty associated with the interferometry to a negligible contribution, in contrast to it being a major uncertainty source in the current systems. The second goal of this project is the practical realization of extremely high accuracy optical reference source can be created in a manner that can be realized in industry.

\section{Customers}

he optical comb will serve internal needs as the top of the traceability chain in PED. There are both internal and external (e.g., DOD) needs for calibration at $543 \mathrm{~nm}$, and the multicolor capabilities of the comb will fulfill this need. State-of-art refractive index measurements will be needed to meet future industrial needs, particularly for the semiconductor industry where refractive index corrections limit our ability to deliver measurements of the highest accuracy. 


\section{MicroFeature Calibration Development}

\section{Challenge/Problem Addressed}

Acro-parts are becoming increasingly important to $\checkmark$ our economy, but systems for their measurement are still in their infancy.

\section{Project Objective}

evelop multiple approaches for high accuracy measurements of small features ( $1 \mathrm{~mm}$ to $50 \mu \mathrm{m})$.

\section{Impact/Benefits}

the he new capabilities have opened up entirely new industries as customers. We have made high accuracy measurements on small nozzles and fuel injectors, center hole of fiber ferrules, very small volume standards, Lithography, Electroplating, and Molding or Lithographie, Galvanoformung, Abformung (LIGA -produced gears, areas of knife edge apertures, and many other artifacts. Measurements of the holes in fuel injectors make it possible to carefully quantify the errors associated with current measurement techniques (e.g., X-ray tomography). Our measurements of knife-edge apertures are the most accurate in the world, providing a path toward improved radiometry standards. Measurements of micro-pivots help to elucidate how form relates to function. Some fiber-probe measurements are illustrated in the section below.

\section{Customers \& Collaborators}

he he most immediate need is for measurement of advanced fuel injectors from manufacturers of internal combustion engines. NIST has also received requests for measurements of micro-optical switching components that we could not perform previously but now have suitable capability; more generally, we hope to serve the needs of the telecom industry or other users of fiberbased micro-optical components. This work was done in collaboration with: WT\&T; Canada (fiber optics); InsituTec; Charlotte NC (CMM probes); MSP Corp Shoreview, MN (small holes); University of Hartford (probes); Optical Technology Division, NIST (knife-edge apertures); Mitutoyo of America (UMAP); DOE (fuel cells); Ceramics Division at NIST (Wolfgang Haller)

\section{Complex Geometry Instrumentation and Standards}

\section{Challenge/Problem Addressed}

omplex surfaces are increasingly employed in
manufacturing, especially for large components. The U.S. is a major supplier of technologies that meet this need; these systems include laser trackers, LADAR (Laser Detection and Ranging), and CMMs used laser probes. Currently there are few calibration facilities and no national or international standards for this class of metrology instruments.

\section{Project Objective}

D rovide access to high accuracy calibrations, artifacts, research facilities, and documentary standards, applicable to large scale complex geometry instrumentation.

\section{Impact/Benefits}

- Completed both the International Standard on coordinate metrology ISO 10360.2 (2009) and harmonized the U.S. with the international community by the development and publication of ASME B89.4.10360-2 (2008) Acceptance test and re-verification test for coordinate measuring machines (CMMs)

- First official NIST laser tracker calibration per the ASME B89.4.19 standard, 2009

- First official NIST articulated arm CMM calibration per ASME B89.4.22, 2009.

\section{Customers \& Collaborators}

he target customers of this project are instrumentation companies that are developing new scanning technology and need access to calibration facilities to determine their actual measurement errors and thus enable improvements in the technology. Collaborators include: FARO Technologies, Kennett Square PA; The Boeing Company; Seattle, WA; Automated Precision Inc, Gaithersburg MD; QuantaPoint Inc, Pittsburgh, PA; Metris Inc., Manassas, VA 


\section{Micrometer level Surface Finish Metrology}

\section{Challenge/Problem Addressed}

S. manufacturing needs improved speed, resolution, and accuracy of surface finish measurements to enable improved productivity. Criminologists need a traceability system for optical inspection devices of bullets and casings in crime laboratories according to recently developed guidelines of the American Society of Crime Laboratory Directors (ASCLD).

\section{Project Objective}

D rovide optical three-dimensional surface topography measurement at the micrometer level. Applications include the traceable measurement of topography of bullets and casings in crime labs, where the fine individual characteristics that produce positive identification of individual weapons need to be separated from longer scale characteristics of overall shape.

\section{Impact/Benefits}

- The NIST Report, Surface Topography Analysis for a Feasibility Assessment of a National Ballistics Imaging Database, was heavily cited by the National Academies in their publication, entitled Ballistic Imaging, one of the reports leading to a substantial ongoing reevaluation of the use of firearms evidence from fired bullets and casings; 2008.

- The On-line Surface Metrology Algorithm Testing System has received thousands of hits by users and has been used in an international comparison of Surface Metrology Software, recently published by NPL (UK).

\section{Customers \& Collaborators}

ational and local forensic laboratories such as the National Laboratory Center of the Bureau of Alcohol, Tobacco, Firearms and Explosives, and the Central Laboratory of Federal Bureau of Investigation; the local ATF, FBI and police laboratories and the National Integrated Ballistics Information Network including 250 forensic laboratories nationwide; International customers include the Federal Criminal Police Office in Germany, the FTI (Forensic Technology Inc.) in Canada and a private company (Spzoocenzin Co.) in Poland. 


\section{PED Program}

\section{Nanomanufacturing Metrology}

Advance U.S. leadership in nanomanufacturing through the development of physical standards traceable to the International System of Units (SI) and through the development of physics-based models and calibration techniques that enable accurate determination of dimensional information.

\section{Staff}

11 NIST Staff

6.5 Guest Researchers/Contractors

\subsection{Total FTEs}

\section{Challenge}

D rovide the calibration techniques and artifacts that realize the Système International d'unités (SI) definition of the meter to meet the most demanding nanoscale industrial needs.

\section{Technical Strategy}

he program aims to provide industry

with accurate and timely dimensional scale metrology at the nanoscale to enhance U.S. productivity and innovation. Fundamental to this objective is meeting the current and anticipated one and two dimensional scale calibration and the SI dimensional traceability requirements for improved yield and product qualification for the constantly evolving nanomanufacturing industry. The fundamental goals for this objective are to provide accessibility to the SI unit of length to the nanomanufacturing industries, such as semiconductor manufacturing, data storage, and photonics, by means of reference metrology instruments and calibration standards that are compatible with industry metrology instrumentation.

\section{Selected Results and Impacts}

- C-AFM used extensively in international comparisons. NIST participated in an international round robin of two dimensional pitch measurements using the C-AFM. Two gratings - $1000 \mathrm{~nm}$ and $290 \mathrm{~nm}$ pitch were measured. Commenced tri-lateral comparison of pitch measurements with A-Star (Singapore) and a commercial standards supplier (ASM) also completed.

- Developed two Reference Metrology Scanning Electron Microscopes (SEMs) equipped with laser interferometry, ready for traceable calibrations of masks, wafers and other samples and characterized all important SEM parameters to minimize measurement uncertainty. 


\section{Selected Publications}

Attota, R., Germer, T., and Silver, R.M., "Nanoscale measurements with a through-focus scanning optical microscope", Future Fab, 30, 2009 p. 83-88.

Silver, R.M., Barnes, B., Attota, R., Jun, J., Stocker, M., Marx, E., and Patrick, H., "Scatterfield Microscopy to Extend the Limits of Image-based Optical Metrology," Applied Optics, Vol. 46, 2007, p. 4248-4257.

\section{Contact Information:}

Richard Silver, Program Manager

Group Leader, Surface and Microform Metrology

Bldg. 220, A119

Gaithersburg MD

301-975-5609

richard.silver@nist.gov

\section{Selected Staff Recognition}

\section{Board Memberships}

\section{Staff}

Richard Silver

James Potzick

\section{Board Memberships}

SPIE Advanced Lithography Executive Committee, 2006-2010

SEMI Standards Microlithography Committee

\section{Leadership}

\begin{tabular}{|c|c|}
\hline Staff & Leadership \\
\hline Richard Silver & $\begin{array}{l}\text { SPIE Advanced Lithography, Organizing committee, } \\
\text { 2001-2008 }\end{array}$ \\
\hline Ronald Dixson & Chair of ISO/TC201/SC9/SG3 \\
\hline Excellence & \\
\hline Staff & Excellence \\
\hline Richard Silver & Elected Fellow of SPIE, 2008 \\
\hline Richard Silver, James Potzick, and Ravikirran Attota & $\begin{array}{l}2008 \text { Dept of Commerce Silver Medal for advances } \\
\text { in optics }\end{array}$ \\
\hline
\end{tabular}


Nanomanufacturing Metrology

Projects

\section{Optical Linescale Metrology}

\section{Challenge/problem Addressed}

the he cornerstone of traceability for the SI Unit of length (the meter) is the Linescale Interferometer (LSI) that provides world-class one-dimensional scale calibrations traceable to the SI. The LSI performance is verified by international intercomparisons, where it consistently performs as one of the top instruments in the world. This facility provides calibrations for customers in the nanomanufacturing industry, such as major metrology tool suppliers. It is also the traceability link for most of the other dimensional metrology instruments within NIST, and more than ten Standard Reference Materials (SRMs) (i.e., 473, 475, 1692, 1960, 1961, 1965, 2059, 2800,5000, 5001), as well as other SRMs in development.

\section{Objectives}

nsure continued operation of the facility (which is more that 40 years old) with reduced uncertainty, and procure a new instrument that substantially decreases the dominant uncertainty due to index of refraction.

\section{Accomplishments}

- The LSI was one of top performing instruments in the Nano3 key comparison.

- The LSI is one of the top instruments in the preliminary results of the Euromet.L-K7 key comparison.

\section{Planned Future Accomplishments}

- Over the past 5 years, the LSI performed 74 calibrations, some of which have very high impact. A single LSI calibration can directly control several hundred million dollars of industrial production around the world, and indirectly control billions of dollars of industrial production. A continued level of calibration performance is a key future deliverable.

\section{Customers and Collaborators}

VLSI Standards, Nikon, Northrop Grumman Space, Pratt \& Whitney, Lockheed Martin, Corning, U.S. DOE, Mitutoyo America, ALCOA, U.S. Customs Lab, IBM, L.S.

Starret, EuroMet, Dupont/Toppan Photomask

\section{Nanometer Scale Dimensional Metrology: Calibrated Atomic Force Microscope}

\section{Challenge/problem Addressed}

he he semiconductor and nanotechnology industries have rapidly increasing dimensional metrology requirements in regimes where traceability to the SI unit of length is not always readily available. For example, the International Technology Roadmap for Semiconductors (ITRS) identifies dimensional metrology as a key enabling technology for the development of next-generation integrated circuits.

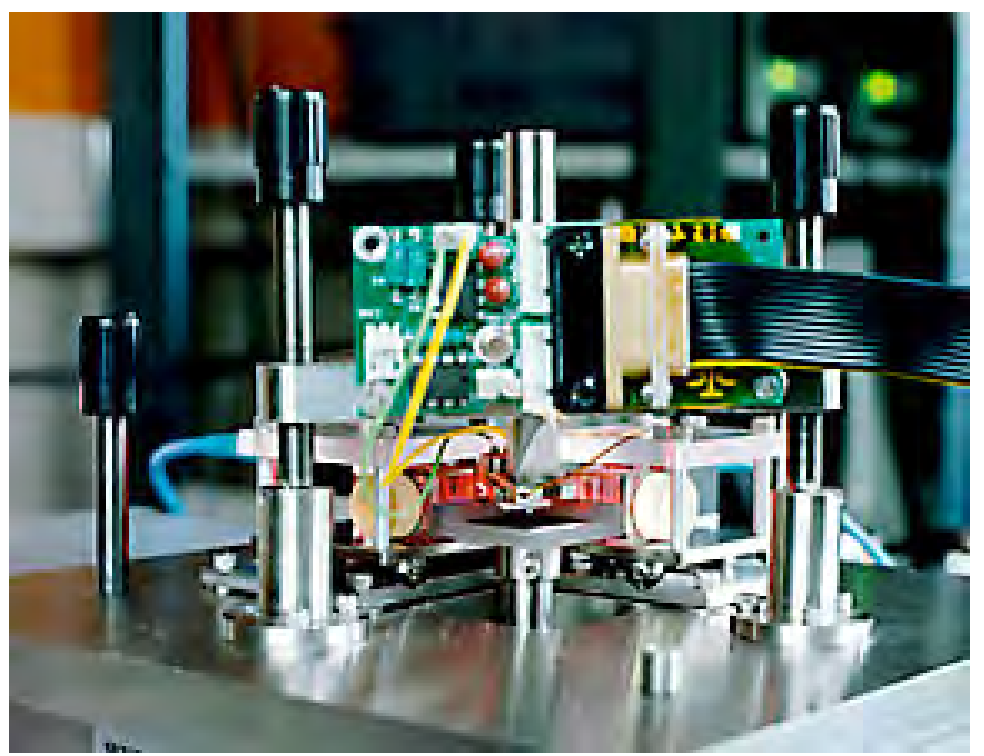

The NIST C-AFM is shown above from a side view in which the head is visible above the specimen platform in the foreground. and the lateral displacement measuring interferometers are visible behind.

\section{Objective}

eet the needs of users of scanned probe micro-
scope (SPM) metrology in the nanotechnology
and semiconductor industries - where relevant fea-
ture sizes continue to shrink and are now approaching
atomic dimensions.




\section{Selected Accomplishments}

- The C-AFM was used to perform a traceable 100 nm grating pitch measurement with $6 \times 10^{-4}$ relative expanded uncertainty in support of SEM metrology of gold nano particles for NIST Reference Material (RM) project.

- Completed NIST participation in an international round robin of two dimensional pitch measurements using the C-AFM. Two gratings $-1000 \mathrm{~nm}$ and $290 \mathrm{~nm}$ pitch - were measured.

\section{Planned Future Accomplishments}

- Traceable nanoscale length measurements for U.S. nanotechnology industries directly or through commercial standards suppliers (e.g. VLSI Standards, K-TEK, Veeco Probes) or consortia (e.g., SEMATECH).

- Perform international comparisons on step height. Work on one and two-dimensional grating period measurements. Perform comparisons of two-dimensional AFM pitch metrology, the C-AFM measurements with relative expanded uncertainty $(\mathrm{k}=2)$ of about $1 \times 10^{-3}$.

\section{Customers and Collaborators}

VLSI Standards, Veeco Probes (Nanodevices), K-TEK,PTB, VLSI Standards, Advanced Surface Microscopy, NRC-INMS

\section{Wafer Level AFM Metrology for Critical Dimension Measurements.}

\section{Challenge/problem Addressed}

o address traceability problems in semiconductor dimensional metrology, NIST in collaboration with SEMATECH developed and implemented a criticaldimension atomic force microscope (CD-AFM) based reference measurement system (RMS). The key goal of the system is to transfer traceable length measurements to tools used in the semiconductor industry.

\section{Objectives}

evelop traceable CD-AFM based dimensional measurement systems for calibration of linewidth, step height, pitch, and related features, and disseminate the measurements and underlying technology to industry.

\section{Accomplishments}

- Introduced and disseminated SI traceable width samples to SEMATECH member companies.

- Disseminated methods for implementing a reference measurement system to U.S. semiconductor industry. Developed of a reference measurement system at SEMATECH.

- Developed a rigorously traceable linewidth calibration system.

\section{Planned Future Accomplishments}

- Continue measurement and development support for SEMATECH metrology program by providing reference measurements for litho metrology projects.

- Develop and disseminate SI traceable methods for CD-AFM-based sidewall angle measurements.

\section{Customers and Collaborators}

VLSI Standards, International SEMATECH, Advanced Metrology Advisory Group, member companies, Dupont/Toppan Photomask, International Technology Roadmap for Semiconductors; Microscopy and Metrology, FEI Co, ISO, E. Fjeld Co. 


\section{Wafer-Level SEM Metrology for Critical Dimension Measurements: SEM Metrology}

\section{Challenge/problem Addressed}

T he U.S. industry needs high-precision, accurate, shape-sensitive dimension measurement methods and relevant calibration standards. The SEM Metrology Project supports all aspects of this need since scanning electron microscopy is key microscopic technique used for this sub-100 nm metrology.

\section{Objectives}

D rovide the microelectronics industry with highly accurate scanning electron microscope measurement and modeling methods for shape-sensitive measurements and relevant calibration standards with nanometer-level resolution.

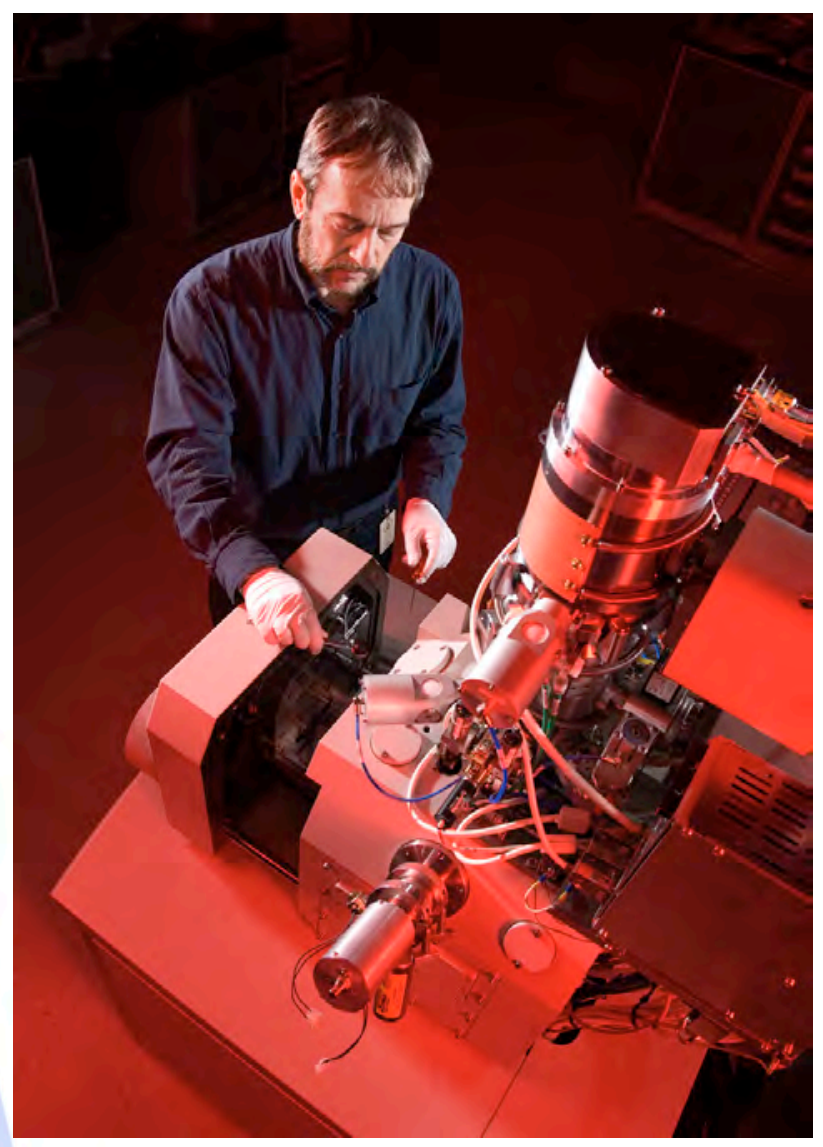

\section{Selected Accomplishments:}

- Developed two Reference Metrology SEMs equipped with laser interferometry, ready for traceable calibrations of masks, wafers and other samples.

- Characterized all important SEM parameters to minimize measurement uncertainty.

\section{Planned Future Accomplishments}

- The Helium Ion Microscopy (HIM) technology is not yet as optimized, developed or as mature as the SEM. As a new technique, HIM is just beginning to show promise and many potentially advantageous applications for integrated circuit and nanotechnology have yet to be exploited.

- Develop models to correctly interpret the signal generation mechanisms and to understand the imaging mechanisms. These are indispensable for accurate nanometer-level metrology.

\section{Customers and Collaborators}

International SEMATECH, Advanced Metrology Advisory Group, International Technology Roadmap for Semiconductors; Microscopy and Metrology Sections, Zeiss/ALIS Corp, FEI Co., ISO, E. Fjeld Co. 


\section{Wafer-Level SEM Metrology for Critical Dimension Measurements: Modeling}

\section{Challenge/problem Addressed}

idth, linewidth, and boundary contours must
generally be determined from an object's image. However, the image is not an exact replica of the line. The scanning electron microscope (SEM), scanning probe microscope (SPM), and optical microscope all have image artifacts that are important at the relevant size scales. These artifacts are related to the probe sample interaction physics and often result in systematic edge assignment errors. These errors may vary due to details of the sample shape, instrument condition, the proximity of neighboring features, charging, contamination, etc. Determinations of width, roughness, and contour position therefore require modeling of the probe/ sample interaction in order to correct image artifacts and identify edge locations.

\section{Objectives}

D evelop and validate instrument function models for scanning electron microscopes and atomic force microscopes, use those models to analyze existing measurement practices, alert industry metrologists to significant unrecognized errors, and suggest measurement practices less subject to error; and use the models to correct images for measurement artifacts, thereby obtaining improved accuracy.

\section{Selected Accomplishments}

- Developed and published an unbiased linewidth roughness metric. Subsequently developed and published (with Applied Materials) a variant of the method that can be used with lines that shrink during measurement, as photoresist does.

- Wrote an SEM simulator that accepts samples of arbitrary 3-D shapes.

\section{Planned Future Accomplishments}

- Collaborate with researchers at the Illinois Institute of Technology to further extend the software capabilities to encompass applications where images, samples, and tips are represented by "dexels" rather than pixels. Dexels may have multiple heights in place of a pixel's single height, thereby allowing reentrant features to be represented.

\section{Customers and Collaborators}

Victor Katsap, Nu Flare Technologies, Maki Tanaka, Hitachi Ltd., Prof. Xiaoping Qian, Illinois Institute of Technology, Veeco Instruments, B. D. Bunday, SEMATECH, Z. J. Ding, University of Science and Technology of China, R. Katz, C. D. Chase, R. Kris, and R. Peltinov, Applied Materials 


\section{Photomask Dimensional Metrology}

\section{Challenge/problem Addressed}

easurement of linewidth or critical dimension
(CD) on photomasks used in the manufacture of semiconductor devices continues to be one of the most fundamental dimensional metrology needs in the semiconductor and nanomanufacturing industries.

\section{Objectives}

- he goal of this project is to provide industry with accurate and timely dimensional metrology at the nanoscale to enhance U.S. productivity and innovation. In particular, to provide accurate critical dimension metrology for photomask features, and traceable to the meter.

\section{Selected Accomplishments}

- Collaborated directly with representatives of the photomask industry and SEMATECH Mask Advisory Steering Council to determine their requirements in the next generation NIST photomask linewidth standard.

- Completed a bilateral intercomparison on photomask linewidth standards between NIST and Physicalishe Technicshe Bundesanstalt (PTB) to ensure agreement between the leading two international suppliers of photomask calibration standards.

\section{Planned Future Accomplishments}

- Develop new measurement techniques to produce sharper edge profiles to reduce linewidth measurement uncertainty. Develop better models to understand the physical process that results in a broadened line edge and with this information we will be able to more accurately automate the assignment physical edge during measurement.

- Develop accurate physics based modeling to deduce the object dimensions from the microscope image. Image modeling will also extend the limits of optical metrology to feature sizes well below the wavelength of light used. This will be based on improved parametric modeling.

\section{Customers and Collaborators}

SEMATECH, Photronics, Toppan Photomasks, Inc., VLSI Standards, IBM, Intel, SEMI International Standards, Physikalisch Technische Bundesanstalt (Germany), BIPM

\section{Overlay Instrument and Wafer Target Designs}

\section{Challenge/problem Addressed}

significant challenge for the semiconductor manu-
facturing industry is to develop advanced metrology techniques for overlay and registration to enable the continued long-term advance of device performance based on the stringent International Technology Roadmap for Semiconductors (ITRS) industry guidelines.

\section{Objectives}

evelop advanced position metrology, techniques and standards for overlay and registration that meet the critical semiconductor industry needs and those future challenges of the demanding nanomanufacturing industry.

\section{Selected Accomplishments}

- One major impact of the overlay project was the incorporation of NIST-developed methods into the hardware tool sets of leading metrology tool manufacturers. Target reversal procedures developed in the Nanomanufacturing program have been installed that help achieve the lowest tool induced shift (TIS) - a measure of tool accuracy for overlay measurement, location in the field of view.

- SEMATECH applied for a joint NIST/SEMATECH patent in Feb, 2008, for the new super-resolution overlay target. These targets have the potential to change the target designs and methodology widely in used by the industry. 


\section{Planned Future Accomplishments}

- Through-focus scanning optical microscope (TSOM) is another new optical method being developed at NIST for nanometrology. A conventional optical microscope is used to collect the images of a given target as it is scanned along the focus axis. Two dimensional TSOM images generated by processing acquired optical images show good promise for demonstration of nanometer sensitivity and accuracy.

- Scatterfield microscopy is one of the methods developed in this project for advanced optical nanometrology based on optical signal analysis rather than optical image processing. This technique is now being investigated for applications in overlay measurements. Arrayed targets with updated designs are now being tested.

\section{Customers and Collaborators}

KLA Tencor, Nanometrics, Nova, AMD, SEMATECH, ISMI, IBM, Intel, SEMI International Standards

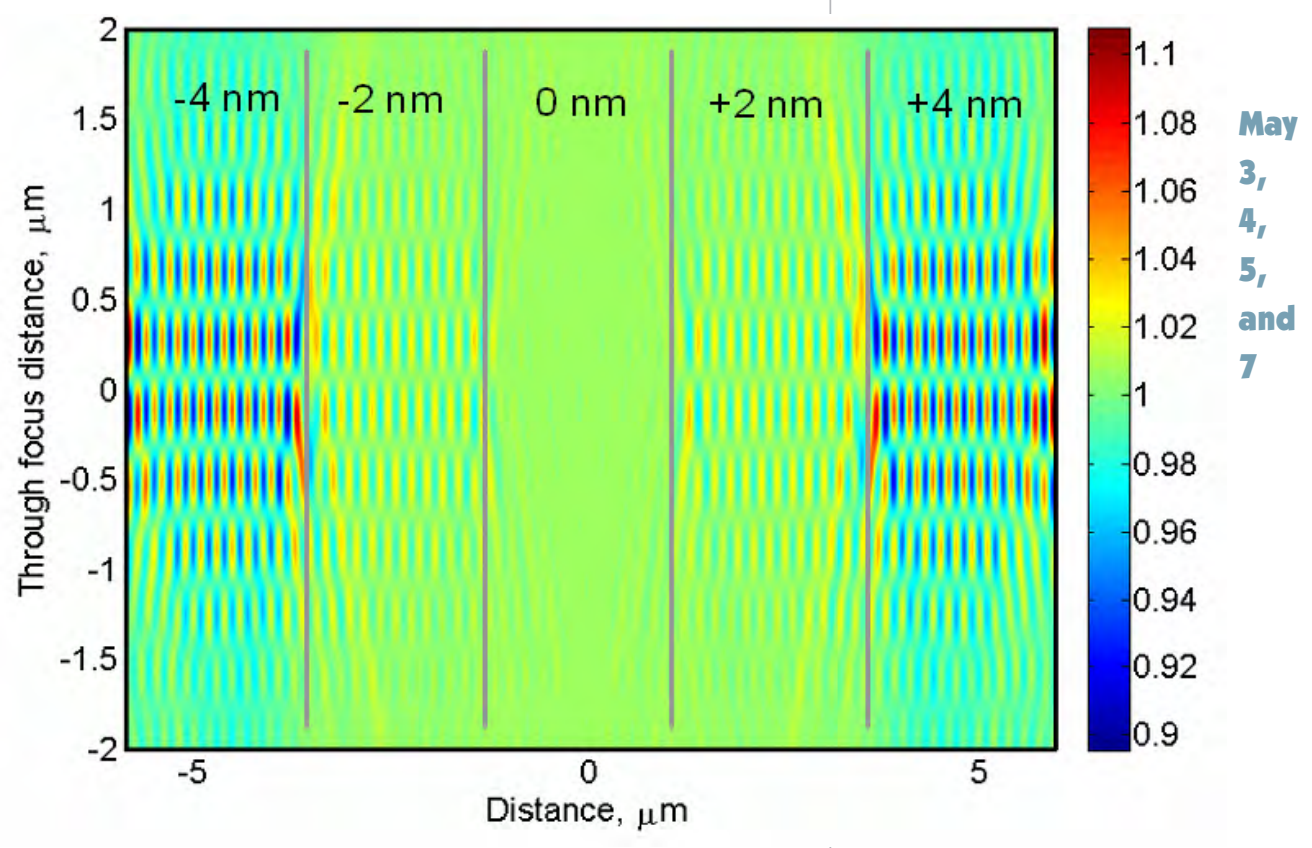

TSOM image of a composite overlay target designed for double patterning showing sensitivity for different overlay values indicated. 


\title{
PED Program
}

\section{Next-Generation Nanometrology}

\author{
Meeting the electronics industry's rallying cry for ever smaller and faster components \\ requires more than improvements in manufacturing techniques: engineers must be \\ able to see and measure what they are building. The limits of conventional imaging \\ have been reached and passed, so NIST is devising new ways to measure and image \\ nanoscale components quickly and accurately.
}

\section{Staff}

5 NIST staff

9 Guest researchers

14 Contractors

14 Total FTEs

\section{Challenge}

- he next generation of nanotechnology manufac-

turing will hit a wall without new measurement techniques to image, analyze, and refine the atomically dimensioned devices now being envisioned.

\section{Technical Strategies}

he program has several strategies to develop meth-

ods to accurately measure nanoscale features. Current measurement techniques are not only slow -- scanning a sample by sending an electron beam back and forth across it, for example -- but often entail damage or destruction of a product to see its interior. Current optical microscopes can rapidly examine objects without doing damage, but can only measure features down to about the wavelength of light. The program will develop advanced optical microscopes using a technique called scatterfield microscopy, which can extract quantitative information from measurements of the angle, polarization and other attributes of light bouncing off a sample.
The program also aims to improve non-optical microscopy. The flagship is the scanning helium ion microscope, a novel instrument related to the scanning electron microscope that scans with a beam of helium ions instead of electrons. The technique has better resolution than scanning electron microscopy, and can also modify a sample by implanting helium ions or milling away material at the surface to reveal additional features.

The program also focuses on measuring nanoparticles, a challenge that is vital to U.S. industry because of nanoparticles' promise for the pharmaceutical industry along with their possible environmental risks. NIST is also investigating the "fate of nanoparticles in biological systems" thus developing the accurate dimensional metrology for the environment, health and safety.

The final program focus represents a jump from NIST's history of creating exact measurement standards to distribute for calibration purposes. Project researchers wish instead to provide measurement methods that allow companies to create their own standards - such as by publishing a "certified" value for some natural standard, like a crystal lattice spacing. Customers could then prepare their own crystal as a calibration standard. 


\section{Selected Results and Impacts}

- Demonstrated imaging and characterization of intact liposome-based targeted nanoparticle delivery systems by scanning probe microscopy in a fluid imaging environment. This paves the way for targeted liposomes to be used in nanomedicine.

- Developed an electron microscope image simulator capable of dealing with complex three-dimensional samples. This helps in inferring dimensions from actual images in new 3D microelectronics and nanomedicine technology.

- Developed in collaboration with other NIST laboratories the new gold nanoparticle size standards: RM 8011, RM 8012, and RM 8013 will provide a method to accurately measure air samples to assess potential health risks.

- Released RM 8820 Multipurpose dimensional metrology standard for calibration of SEMs for all applications.

\section{Selected Publications}

Dagata, J.A.; Farkas, N.; Dennis, C.L.; Shull, R.D.; Hackley, V.A.; Yang, C.; Pirollo, K.F.; Chang, E.H., "Physical characterization methods for iron-oxide contrast agents encapsulated within a targeted liposome-based delivery system," Nanotechnology 19, 305101, 2008

\section{Cizmar, P.; Vladar, A. E.; Postek, M. T., "Optimization of Accurate SEM imaging by use of artificial images" 2009 SCANNING/SPIE Proceedings 7378:737815-1 - 737815-6, 2009}

Gorman, J. J.; Balijepalli, A.; and LeBrun, T. W.; "Control of Optically Trapped Particles for Brownian Motion Suppression." Accepted to be published in the IEEE Transactions on Control Systems Technology, 2009

\section{Contact Information:}

\section{John Kramar, Program Manager}

301-975-3447

john.kramar@nist.gov

http://www.nist.gov/mel/ped/nm/ngn.cfm

\section{Selected Staff Recognition}

\section{Board Memberships}

\section{Staff}

M.T. Postek

A. Vladar

\section{Leadership}

\section{Staff}

J.A. Dagata

\section{Board Membership}

Nanotechnology Briefs Board of Directors

SPIE Advanced Lithography Advisory Board

\section{A. Vladar}

\section{Leadership}

Organizing committee, National Science Foundation (NSF)sponsored workshop on Grand Challenges for Bio-Nano Integrated Manufacturing, Arlington VA April 14-16, 2008

ISO TC 204 SEM Image Sharpness Metrology Standard, 2009

\section{Excellence}

\section{Staff}

R. Attota and R.M. Silver

R.M. Silver

\section{Excellence}

Nano 50 Award for Scatterfield Optical Microscopy

DOC Silver Medal, "for development of optical methods in overlay metrology." 2008 


\section{Next-Generation Nanometrology Projects}

\section{Scanning Particle-Beam Microscope Innovations}

\section{Challenge/Problem Addressed}

anotechnology is already facing dimensional measurement and characterization challenges that far exceed the capabilities of current measurement science and techniques. Three-dimensional nanometer-scale objects and features must be measured with unprecedented precision and uncertainty, and with high throughput.

\section{Objectives}

D evelop accurate methods for the characterization and three-dimensional (3D) metrology of nanoscale materials and features. This is becoming more and more necessary as, for example, new microelectronics technologies and nanomedicine innovations extend into the vertical dimension.

\section{Selected Accomplishments}

- Developed He ion lithography and nanomilling methods with approximately $10 \mathrm{~nm}$ smallest fabricated structures. These are the finest features ever nanomilled, and open up new possibilities for innovation.

- Developed a strong cooperation with Zeiss regarding the optimization and implementation of HIM. NIST expertise is currently being used to improve the performance of the HIM to make it a viable alternative to SEM for nanotechnology and nanomanufacturing.

\section{Planned Future Accomplishments}

- Optimize and develop new image capture and averaging techniques to enhance the performance for SEM and HIM imaging by developing within frame drift correction.

- Characterize the sensitivity of the HIM for high-resolution metrology of linewidths in silicon, photoresist, and mask materials, to qualify the potential of HIM as a metrology method for next-generation nanoelectronics.

\section{Customers and Collaborators}

International SEMATECH, Advanced Metrology Advisory Group; International Technology Roadmap for Semiconductors, Microscopy and Metrology Sections; Zeiss/ALIS Corp.; FEI Co; Hitachi Co.; E. Fjeld Co.

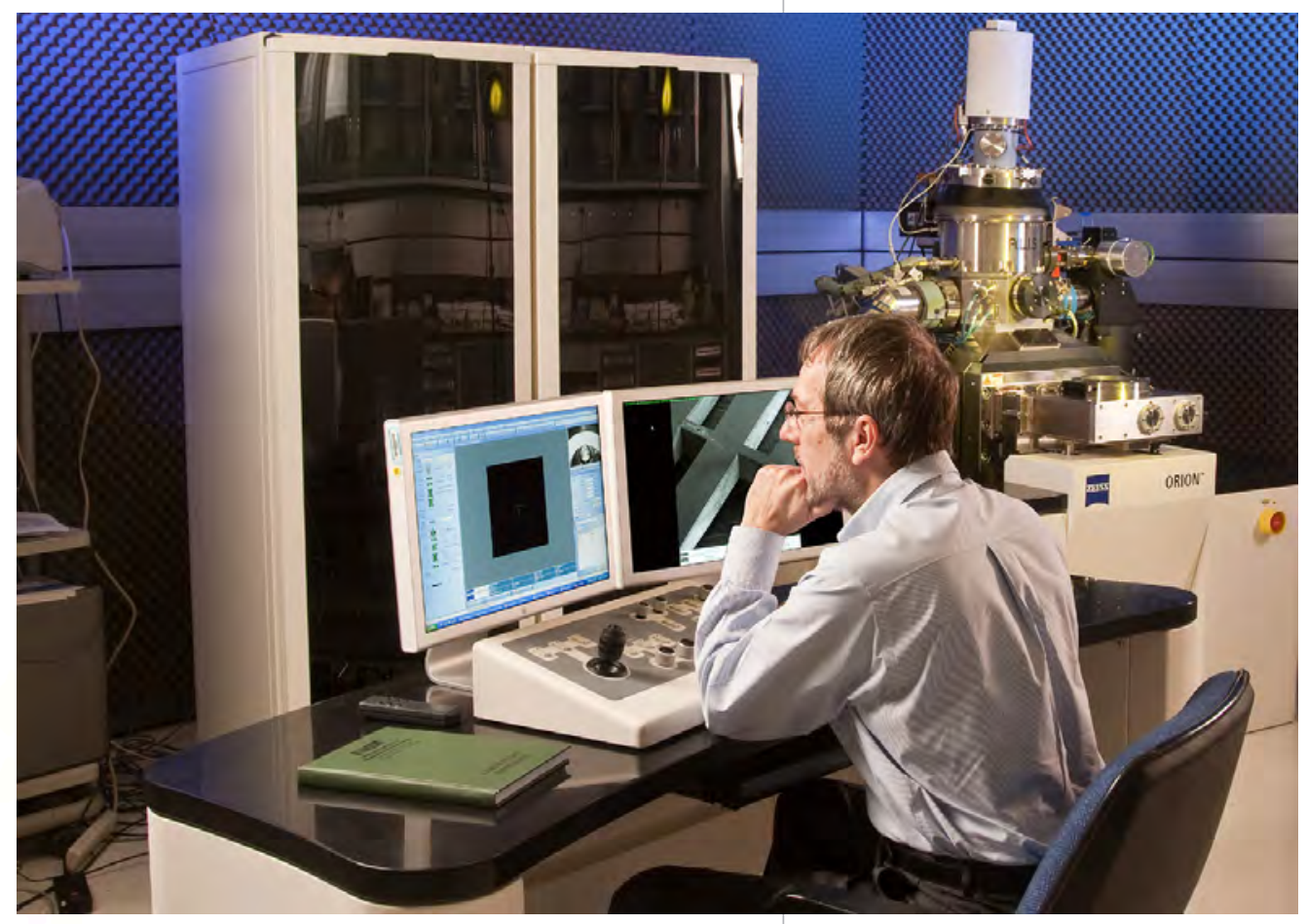

Helium Ion Microscope 


\section{Atomic Force Microscope Nanoparticle Metrology}

\section{Challenge/Problem Addressed}

- his project is working to enable a metrological basis for optimized formulation and regulatory approval of diagnostic and therapeutic nanoparticle formulations. Without consistent measurement methods and a correlation between physical and biological assays, new nanomedicines face a huge hurdle to human use.

\section{Objectives}

D rovide new measurement methodologies that track the formulation, manufacture, and bio-distribution of nanoparticles.

\section{Accomplishments}

- Demonstrated optimized methods for immobilizing intact liposome-based nanoparticle delivery systems (NDS) for scanning probe microscope (SPM) imaging and characterization under fluid conditions, a critical first step for quantitative measurements.

- Evaluated improved magnetic coatings for fluid and dry magnetic force microscopy (MFM) for characterizing dispersed and aggregated magnetic resonance imaging (MRI) contrast enhancement agents. Improving the contrast agents increases sensitivity for imaging smaller features, like incipient tumors.

\section{Planned Future Accomplishments}

- Develop complementary, multiple-technique protocols for characterizing the mean size and size distribution of soft nanoparticles, combining the unique strength of dynamic light scattering, fluid scanning probe microscopy, and electron and optical microscopy.

- Demonstrate methods for quantitatively examining membrane dynamics and nanoparticle uptake, motion, and eventual fate in living cells. This information is critical for understanding how nanoparticles may damage living cells, or may be used as therapeutic nanomedicine agents.

\section{Customers and Collaborators}

U.S. National Cancer Institute (NCI), Bethesda MD, U.S. Food \& Drug Administration, White Oak, MD, Georgetown University Medical Center (GUMC), Washington DC, Aparna Biosciences, Rockville MD, Nanotechnology Characterization Laboratory (NCL), Frederick MD, University of Akron, Akron $\mathrm{OH}$, National Institute of Allergy and Infectious Diseases (NIH), Rockville MD 


\section{Nanoparticle Manipulation and Metrology}

\section{Challenge/Problem Addressed}

anoparticles are the most mature sector of the rapidly growing nanotechnology industry, but methods to select and fully manipulate nanoparticles are still rudimentary or nonexistent. This limits development of nanofabrication processes at every step from sorting and characterizing nanomaterials to prototyping and testing functional nanodevices assembled from heterogeneous components.

\section{Objectives}

dvance basic nanomanipulation technology to the
point that 2D and 3D nanostructures can be rapidly
prototyped and tested to enable efficient development of
functional nanodevices.

\section{Selected Accomplishments}

- Developed an integrated scanning trap system for fast 6D manipulation of complex nanoparticles. This first-in-the-world capability is an important step for nanostructure assembly with optical tweezers.

- Demonstrated broad applicability of trapping and instrument performance by trapping a wide range of particles including nanowires, and simultaneously trapping red blood cells and pharmaceutical nanoparticles. Trapping multiple objects simultaneously is necessary for complex assembly.

\section{Planned Future Accomplishments}

- Demonstrate robust trapping of sub-100-nm nanoparticles using high-speed, intelligent servo control systems to modulate the position of the optical trap based on the observed disturbances and the prior knowledge of the detailed trap-particle interaction potential. This will bring optical trapping down to the realm of true nanoparticles.

- Demonstrate the unique value of true, individual nanoparticle trapping capability for controlled nanomanufacturing. An initial example could be a reference material consisting of an ordered array of nanoparticle defects.

\section{Customers and Collaborators}

National Cancer Institute (NCI), Nanotechnology Characterization Laboratory (NCL); University of Maryland; Korea Institute of Science and Technology (KIST), Korea; University of Western Michigan; Purdue University 


\section{Molecular Measuring Machine $\left(M^{3}\right)$}

\section{Challenge/Problem Addressed}

he emerging nanotechnology industry is in need of nanometer-accurate two-dimensional position metrology of nanometer-scale objects and features.

\section{Objectives}

Dovide the ultimate metrology capability for feature placement and two-dimensional position metrology over macroscopic distances. This instrument will then serve as the traceability link for industry metrology instruments via artifacts that will be calibrated on $\mathrm{M}^{3}$, or through the identification and validation of intrinsic standards and the publication of accepted values and best practices.

\section{Selected}

\section{Accomplishments}

- Developed a compact, compound actuator and sensor for controlled metrological motion and measurement. This actuator is a novel design that incorporates a $3 \mathrm{~mm}$ range coarse motion and an $8 \mu \mathrm{m}$ range, guided, fine motion actuator with a capacitance gage sensor, all within a $(25 \mathrm{~mm})^{3}$ volume.

- Developed a method to perform high resolution heterodyne interferometry in vacuum using fiber optic beam delivery. The heterodyne beams are separated for traversal through the fiber optic to avoid mixing and the consequent periodic non-linearity, and then recombined in vacuum. Fiber optic delivery minimizes errors from beam pointing stability and air turbulence.

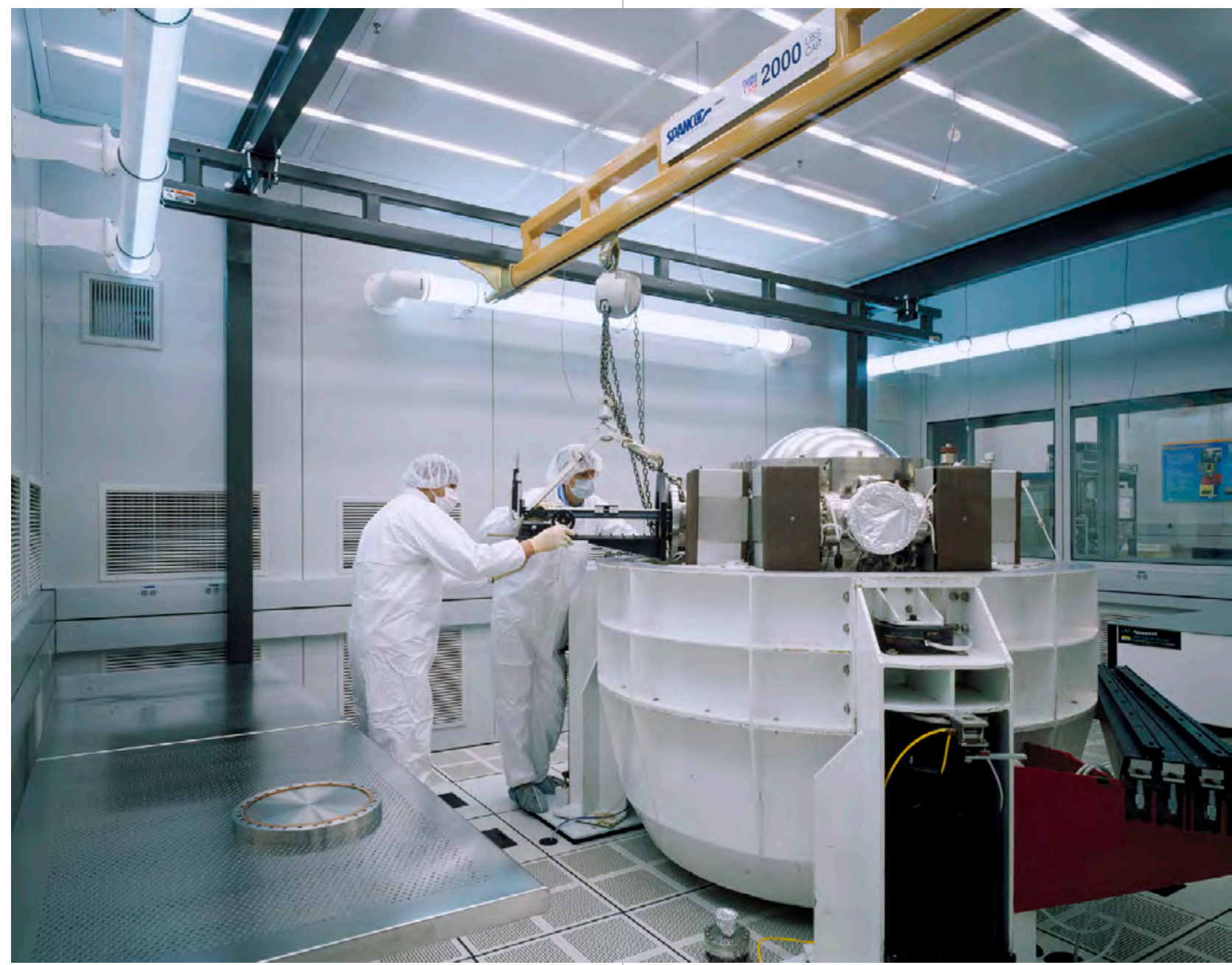




\section{Planned Future Accomplishments}

- Measure an atomic-resolution lattice over an area of a few optical fringes in $X$ and $Y$ to characterize the interferometer metrology system with reference to the crystal lattice.

- Utilize the demonstrated sub-nanometer-level, closedloop control capabilities of $\mathrm{M}^{3}$ with a suitable surface modification or nanowriting technique to produce highly perfect nanoscale calibration standards and experimental test samples.

\section{Customers and Collaborators}

Center for Space Research, Massachusetts Institute of Technology, National Aeronautics and Space Administration (NASA), George Washington University, National Taiwan University

\section{Atom-based Dimensional Metrology}

\section{Challenge/Problem Addressed}

Nim anometrology will play a key role in addressing the challenges in the upcoming nanotechnology industry. There is a need for specialized metrology tools and processes to meet these requirements. The ultimate limit for nanoscale length metrology is the development of intrinsic calibration standards, where the reference dimensions are based on atom spacing within an ordered, crystalline lattice.

\section{Objectives}

evelop intrinsic calibration standards based on the crystalline lattice and to develop the underlying measurement science needed to enable parallel, highthroughput, atomically-precise manufacturing for NIST standards development and for a variety of industrial applications. The current focus is on developing methods to prepare photo-lithographically patterned 3D structures in silicon substrates appropriate for atom counting; and to achieve parallel high-throughput atomically-precise manufacturing (APM) using a multitude of parallel Scanning Tunneling Microscopy (STM) tips simultaneously.

\section{Selected Accomplishments:}

- Awarded a five year, three phase DARPA contract to conduct collaborative research in atomically precise positioning, patterning and metrology, validating the applicability and potential value of this work.

- Designed and built a five chamber ultra high vacuum (UHV) facility regularly capable of producing $1 \times 10^{-8}$ Pa base pressures for performing nanometer scale metrology on various metallic and semiconductor surfaces. 


\section{Planned Future Accomplishments}

- Develop intrinsic calibration standards for pitch and linewidth based on atom-spacings in the crystal lattice. This will bring accurate dimensional standards down into the nanoscale range, necessary for nanomanufacturing.

- Develop tip processing procedures involving field evaporation and thermal processing to consistently obtain tips from a variety of materials that are capable of achieving atomic-scale resolution images in the STM, and capable of consistently effecting atomicallyprecise surface modification.

\section{Customers and Collaborators}

SEMATECH, DARPA, Zyvex Labs Inc., Intel, University of Maryland, University of Illinois at Urbana Champagne, University of Texas at Austin, University of Texas at Dallas

\section{High-Throughput Nanometrology with Scatterfield Microscopy}

\section{Challenge/Problem Addressed}

anomanufacturing requires innovative high-

throughput, non-contact metrology methods to minimize defects, quantify critical dimensions, and sample large areas effectively for maximum yield. This presents a tremendous challenge, to quantify features and identify defects at the nanometer scale over areas ranging from $1000 \mu \mathrm{m}^{2}$ to $1 \mathrm{~m}^{2}$ in dimension.

\section{Objectives}

evelop an ensemble of optical microscopy techniques, termed scatterfield microscopy, that enable dimensional metrology beyond traditional optical resolution barriers. The goal is to enable the cost-effective mass-production of nanotechnology products through accurate, production capable metrology solutions.

\section{Accomplishments}

- Constructed a dedicated platform for scatterfield microscopy $(\lambda=450 \mathrm{~nm})$.

- Quantitative agreement achieved between rigorous modeling and experimental data. Published results demonstrate quantitative agreement for scatterfield measurements of densely arrayed $100 \mathrm{~nm}$ sized lines which vary in $1 \mathrm{~nm}$ increments, validating the utility of optical metrology methods for far sub-wavelength features. 


\section{Planned Future Accomplishments}

- Expand the use to scatterfield microscopy techniques to new areas, such as characterizing catalyst loading and catalyst particle size on membranes used in fuel cells. This will provide a critically-needed high-throughput process control measurement method.

- Demonstrate the capability of scatterfield microscopy for quantitative measurements of defects, nanoparticles, and nanometer-accuracy overlay measurements. This will extend the used of optical measurement methods into the next generation of nanoelectronics manufacturing.

\section{Customers and Collaborators}

KLA-Tencor, Nanometrics, Zyvex, Applied Materials, SEMATECH, Intel, IBM, University of Maryland

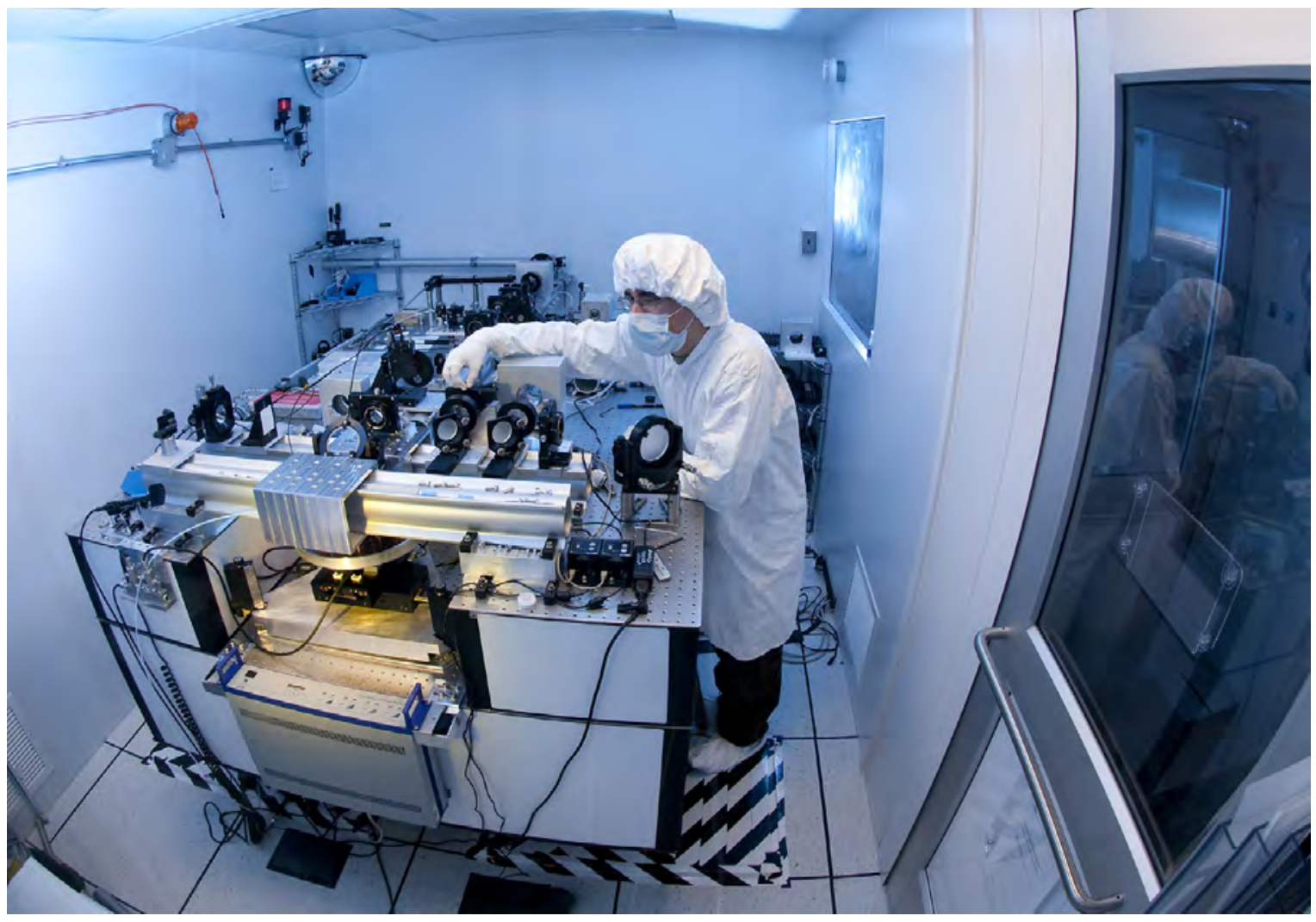

193 nm Scatterfield microscope 


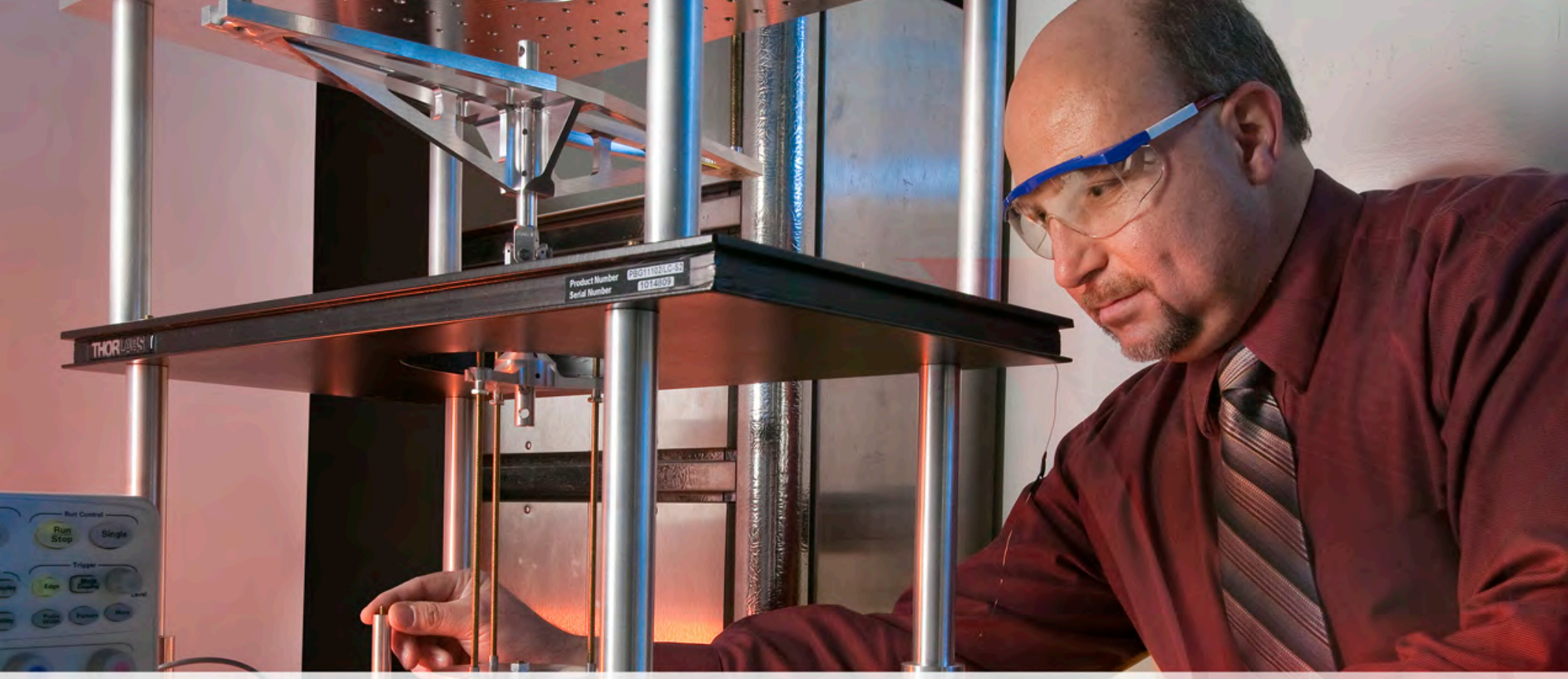

\section{Fulfilling the measurements and standards needs of the United States in mechanical metrology and advanced manufacturing technology}

\section{Manufacturing Metrology}

\section{Overview}

$-\mathrm{o}$ fulfill its mission and to respond to the needs of U.S. industry and government, the Manu-

facturing Metrology Division (MMD) has organized its technical work into three programs.

The three MEL programs led by MMD are:

- Mechanical Metrology

- Metrology for Advanced Optics

- Measurements and Standards for Science-Based Manufacturing

The division consists of a strong team of scientists, engineers, technicians, and support staff, including post-doctoral fellows, full-time guest researchers, and part-time students. The division is fortunate to have staff with expertise and skill in many disciplines, including mechanical engineering, electrical engineering, applied mechanics, physics, optics, acoustics, computer science, and chemistry. Division staff contribute to technical advancements and industry impacts in all six of the MEL core competence focus areas: mechanical metrology, dimensional metrology, nanomanufacturing metrology, manufacturing systems, manufacturing processes, and manufacturing equipment.

Several drivers for U.S. manufacturing have influenced the selection, direction, and content of the MMD programs, in particular:

- Growth of international trade

- Global and distributed manufacturing

- Continual push for higher quality, better performing, customized products
Chief:

Kevin Jurrens (Acting)

NIST

100 Bureau Drive

Stop 8220

Gaithersburg, MD

20899-8220

Phone: 3019756600

Fax: 3018693536

Email:

kevin.jurrens@nist.gov 
- Pressures to increase productivity and reduce costs

- Increased pace of technological change

Through frequent interactions and collaborations, the division ensures that our measurements and standards activities address the needs and priorities of our customers. Informed by these interactions, the MMD programs are structured to respond to key, high-priority manufacturing industry needs that result from the above drivers.

\section{Mission}

T o fulfill the measurements and standards needs of the United States in mechanical metrology and advanced manufacturing technology by:

- conducting research and development in realizing, maintaining, and disseminating SI mechanical units (mass, force, acoustics, vibration);

- developing methods, models, sensors, and data to improve metrology, machines, and processes;

- providing services in mechanical metrology, machine metrology, process metrology, workpiece property metrology, and sensor integration; and

- leading in the development of national and international standards.

In addition, MMD provides service to other federal government agencies to address problems and needs that leverage NIST expertise, facilities, and capabilities.

\begin{tabular}{|c|c|}
\hline gram & Manufacturing Industry Need \\
\hline $\begin{array}{l}\text { Mechanical } \\
\text { Metrology }\end{array}$ & $\begin{array}{l}\text { - Measurement traceability to the SI } \\
\text { - Compliance with regulatory requirements } \\
\text { - Harmonized international standards that reflect U.S. needs } \\
\text { - Improved measurement accuracy, precision, and efficiency } \\
\text { - Capability to cost-effectively manufacture higher quality and/or more complex parts } \\
\text { and assemblies }\end{array}$ \\
\hline $\begin{array}{l}\text { Metrology for } \\
\text { Advanced Optics }\end{array}$ & $\begin{array}{l}\text { - Measurement traceability to the SI } \\
\text { - Harmonized international standards that reflect U.S. needs } \\
\text { - Improved measurement accuracy, precision, and efficiency } \\
\text { - Ability to make new and more complex measurements } \\
\text { - Capability to cost-effectively manufacture higher quality and/or more complex parts } \\
\text { and assemblies }\end{array}$ \\
\hline $\begin{array}{l}\text { Measurements } \\
\text { and Standards for } \\
\text { Science-Based } \\
\text { Manufacturing }\end{array}$ & $\begin{array}{l}\text { - Harmonized international standards that reflect U.S. needs } \\
\text { - Ability to make new and more complex measurements } \\
\text { - Capability to cost-effectively manufacture higher quality and/or more complex parts } \\
\text { and assemblies } \\
\text { - Model-based manufacturing systems and processes } \\
\text { - Integration and management of manufacturing systems, processes, and equipment } \\
\text { - Predictable, well-characterized manufacturing processes, equipment, and systems } \\
\text { - Flexible, reconfigurable manufacturing systems }\end{array}$ \\
\hline
\end{tabular}




\section{Staffing Challenges}

T he primary challenge in effective management of MMD staffing and financial resources arises from the division's broad, multi-faceted technical mission. In each of the division's technical areas, MMD staff supply world-class expertise, experience, and knowledge. However, staffing numbers in each area are relatively small, particularly in comparison to National Measurement Institutes in other countries or to parts of NIST that have a more singular mission. The division's challenge is to meet today's demands while also preparing resources and technical expertise for the future. Future retirements and staff departures must be constantly kept in mind, since the specialized metrology and standards expertise required by MMD are not readily available and may take years for newlyhired staff to develop. The typical MMD metrology expert has deep knowledge in a very specialized field; crossover of the specialized expertise to different areas of metrology even within the division - is rare. Currently the division's professional staffing is very lean, with some metrology areas having only one or two permanent employees. High-quality guest researchers and contractors provide tremendous benefit. MMD continues to encourage and pursue additional NRC post-doctoral researchers for the division.

\section{Facilities and Equipment}

MD manages technical laboratories in several $\checkmark$ buildings across the NIST campus. NIST's newest building, the Advanced Measurement Laboratory (AML), provides world-leading environmental control for air quality, temperature, humidity, vibration, and electrical shielding - all critical for MEL precision measurement capabilities. MMD facilities typically include one or more of the following characteristics:

- Single-purpose facilities that are too expensive for one company to develop or maintain

- State-of-the-art metrology and research

- Support for next-generation instruments or technology

- Provision of effective access to NIST equipment and expertise

- Neutral location for industry competitors to come to develop tests and standards that benefit the whole industry

MMD was fortunate to receive approximately $\$ 2 \mathrm{M}$ of the American Reinvestment and Recovery Act (ARRA) funds provided to NIST for procurement of new state-of-the-art equipment. These funds were used to purchase new mass standards required to meet industry needs in large mass calibrations.

\section{Facilities}

\section{Mechanical Metrology Program}

- Mass Metrology and Standards Facility: Home of the U.S. National Standard for the Kilogram. Custom clean-room environment that provides the U.S. measurement system with the most accurate calibrations available, traceable to the national standard of mass. 
- Small Force Metrology Laboratory: Worldleading facility devoted to measuring forces as small as $10^{-8} \mathrm{~N}$ - the force needed to break three covalent bonds. Application to measurements of coatings, thin films, magnetic disk-drive spring constants, and future mechanical nanodevices.

- Low Frequency Vibration Laboratory: Environmentally-controlled facility devoted to nextgeneration low-frequency vibration metrology $(\sim 0.2 \mathrm{~Hz}$ to $200 \mathrm{~Hz})$ to meet increasing customer needs for traceable calibrations of accelerometers in this range.

- Force Metrology Laboratories: Custom-built, multi-story facility that houses force metrology deadweight machines for SI-traceable calibration of force sensors (load cells, proving rings). Home of the 4.45 MN (1 million lbf) deadweight machine - the world's largest machine with the ability to perform direct, high-accuracy calibration of high-load force sensors.

- Acoustic Anechoic Chamber: A large, well-characterized, ultra-quiet chamber used to precisely characterize sound sensors (hearing aids, sound level meters, microphones) and to perform highprecision acoustic measurements.

\section{Metrology for Advanced Optics Program}

- Extremely Accurate Calibration Interferometer (XCALIBIR): A unique multi-configuration, phase-shifting interferometry system with an aperture of $300 \mathrm{~mm}$ that can perform demanding measurements and calibrations of optical figure, wavefront, and radius of curvature. The instrument is located in a class 1000 clean room with temperature control to \pm $0.02{ }^{\circ} \mathrm{C}$.

- Infrared Interferometer (IR $\left.{ }^{3}\right)$ : Custom-designed infrared interferometer with an aperture of $300 \mathrm{~mm}$ for measuring the thickness variation of silicon wafers.

- Geometric Measuring Machine (GEMM): Custombuilt experimental system for estimating the form errors of aspheric and freeform surfaces from measurements of local curvature.

- Wyko 6000 Interferometer: Commercial phaseshifting interferometer with an aperture of $150 \mathrm{~mm}$ for measurement and calibration of optical elements. 


\section{Measurements and Standards for Science-Based Manufacturing Program}

- DMG 5-axis High-Speed Machining Center: Research platform for machine tool metrology, process metrology, and next-generation machine control. Shared resource with the MEL Fabrication Technology Division.

\section{- Makino 4-axis High-Speed Machining Center:} Research platform for high-speed machining. Shared resource with the MEL Fabrication Technology Division.

- Edgetek Machining Center: Instrumented testbed for measuring process phenomena (e.g., cutting forces, temperatures, chip formation) during orthogonal cutting. The testbed has a unique high-speed, dualspectrum (infrared and visible) micro-videography system for measuring material flow and temperature distribution.

- Mazak 5-axis Turning Center: Research platform for machine tool metrology, process metrology, and smart machining concepts.

- Pulse-Heated Kolsky Bar Facility: A unique, NIST-built device that measures the stress-strain properties of materials undergoing rapid heating and rapid increase of strain. Such data improves models for high-speed machining processes, ballistic impacts, and structural failure.

- Smart and Wireless Sensors Laboratory: A unique facility for testing reference implementations of interoperability standards for smart and wireless sensors.

- Micro-Machining Laboratory: Research facility for development of new metrology concepts relevant to meso-scale machine tools for fabricating micro- and meso-scale parts.

- Diamond Turning Laboratory: Moore M-18 3-axis diamond turning machine for high-precision machine tool metrology and machining process metrology.

\section{Facilities and Equipment Challenges}

The division's existing laboratory facilities are generally sufficient to meet today's customer needs.

Aging of the NIST general purpose laboratories and their environmental systems remain a liability for NIST (and the division) to continue to serve our customers and to meet future, higher-precision metrology demands. Divisions with space outside of the AML will likely need future investments in specialized laboratory renovations to meet ever-increasing environmental requirements for state-of-the-art metrology. Another challenge for the division is to upgrade calibration system equipment and components to take advantage of current technologies while maintaining complete traceability and confidence in measurements for customer calibrations.

\section{Laboratory Safety}

ince the last visit of the NRC Assessment Panel,

NIST has undergone a substantial effort to transform the safety culture of the organization and to implement new safety procedures. As part of this change, MMD has completed a very thorough hazards assessment of our laboratory and office spaces. Many new safety procedures are now in place, and lab-specific safety information has been developed for each MMD laboratory. All MMD managers have taken significant amounts of safety training, including the week-long Occupational Safety and Health Administration (OSHA) 6000 training and hazards recognition. Division staff have received task-specific safety training as needed for their work, such as for laser safety, chemical safety, crane safety, fall hazards, and safe handling of gas cylinders. Safety improvements and reviews are ongoing, with strong support from division staff and managers. 


\section{Metrology for Advanced Optics}

From projecting computer chip designs onto silicon wafers to imaging remote galaxies, advanced optics are crucial to modern technology and science. As the demand for sophisticated optical components increases, so does the need for versatile and accurate inspection methods. NIST works with industry, universities, and government agencies to improve measurement methods for precision optical surfaces, to develop metrology for emerging nano-structured optics, to improve international measurement standards, and to calibrate optical reference artifacts. Program results contribute to innovations in the application and manufacture of advanced optical elements and precision surfaces.

\section{Staff}

2 NIST staff

3 guest researchers

5 total FTEs

\section{Challenge}

$T^{\text {them }}$ he program addresses infrastructural metrology needs of the U.S. optics and photonics industry for the manufacture of precision optical elements possessing high added-value. The optics and photonics industry is a vibrant part of the U.S. economy. Advanced optical and photonic components enable product innovations in many high-technology areas, ranging from medical technology and defense to consumer electronics and astronomy. Optical and photonic technologies furthermore boost competitiveness and technological leadership in manufacturing. "Light is the tool of the future", as evidenced by the growth in machine vision, optical inspection, and laser systems for material processing. Advances in nano-scale and semiconductor manufacturing continue to depend on advances in optical technologies, such as optical projection lithography.

Both the manufacture and development of advanced optical components critically depend on the ability to measure their performance. Only optical surfaces that can be measured can be made. This is particularly important for imaging systems performing at the diffraction limit imposed by the wave nature of light, be they very large optical telescopes with apertures of many meters, or small cameras in mobile phones which must achieve good imaging performance in a tiny space.

Advanced optical systems incorporate features that yield vastly improved performance but pose significant measurement challenges. Examples of such features are complex surfaces, i.e., surfaces that are neither flat nor spherical, micro- and nano-scale surface structures, extreme accuracies, special materials and coatings, and adaptive technologies. The development and manufacture of these advanced features depends on advances in traceable metrology for optical figure and wavefront. In high-impact applications, such as semiconductor lithography, the required accuracies are at demanding subnanometer levels. Traceability requires standards-compliant uncertainty statements that are rare in the optics industry, but increasingly mandated for ISO-certified quality systems and export. No general, widely-recognized, validated way exists to calibrate complex optical surfaces or the performance of nano-structured optics, and the application range and uncertainty of existing methods are poorly understood. This is a measurement barrier to the widespread manufacture and adoption of these optical elements, despite their ability for product innovations.

\section{Technical Strategies}

- he program seeks to promote innovations in the

application and fabrication of advanced optical elements by providing methods, services, and standards for SI-traceable metrology of optical figure and wavefront. The program focuses on optical interferometry, the most 
widely used measurement method for precision optics and surfaces, because no other method can achieve the same versatility and accuracy. The program addresses the following infrastructural metrology needs:

1. Methods that address the challenge of measuring the performance of the rapidly evolving class of nanostructured optics and its relation to fabrication errors.

2. Methods that address the challenge of measuring the shape of complex optical surfaces (rotationally symmetric and freeform).

3. Measurement methods and reference artifacts that address future requirements of the semiconductor industry in the areas of silicon wafer flatness and extreme ultraviolet lithography (EUVL) optics.

4. International comparisons of measurement capability and harmonized standards that ensure effective access to international markets by the U.S. optics industry.

5. Measurement methods and services for flatness, sphericity, and radius of curvature of optical reference surfaces required for interferometric measurements of precision surfaces and optical elements.

The program concentrates on the development of infrastructural measurement methods and services that: 1) promote innovations in a wide range of optical and photonic applications; 2) have a high impact on the U.S. metrology chain; 3) provide a generic approach to characterize and reduce measurement uncertainties; and 4) remove controversy in commerce over measurement results and specification compliance. The program has a strategic focus on techniques and protocols that allow users to make traceable measurements in their own facilities. Where possible, the program seeks to eliminate the requirement of calibrated transfer artifacts through the development of absolute calibration methods. The program develops uncertainty statements, compliant with the Guide to the Expression of Uncertainty in Measurement (GUM), for typical measurement set-ups. The program contributes to the ANSI Optics and Electro-Optics Standards Council Technical Advisory Group to improve standards developed by the ISO/ TC172 Technical Committee on Optics and Photonics. Research in new areas, such as nano-structured optics, is conducted in collaboration with partners and customers working on leading-edge applications.

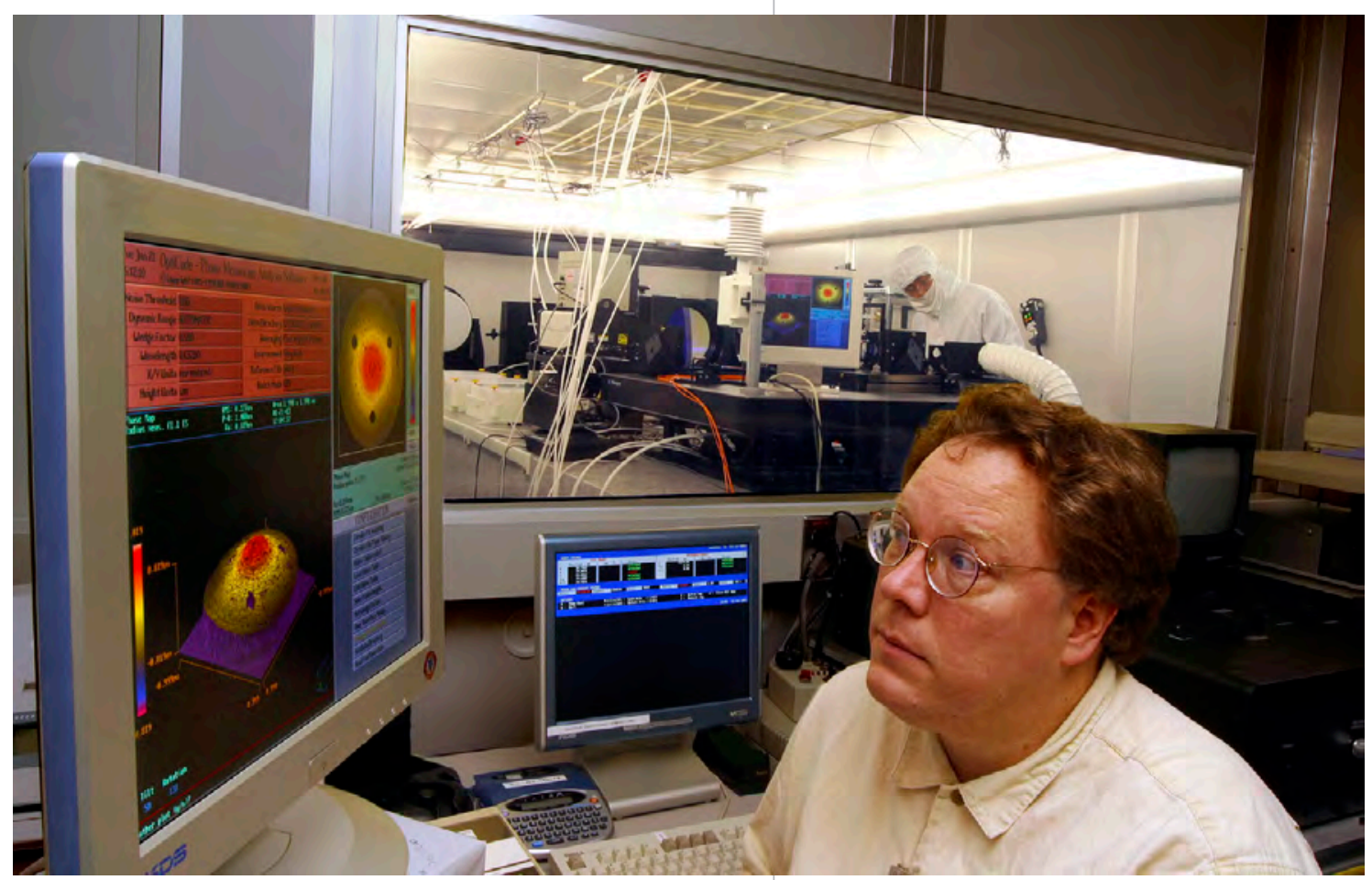

The NIST XCALIBIR reconfigurable interferometer for the measurement of ultra-precision flat, spherical, and aspherical optical elements and precision surfaces. 


\section{Results and Impacts}

- Developed a new method for deformation-free flatness measurements of thin optics, such as photomask blanks for extreme ultraviolet (EUV) lithography. In the method, the optic is floated on a liquid with a high specific gravity. This eliminates mounting induced deformations, and the measured flatness error results only from fabrication errors and coating stresses. At the request of SEMATECH, flatness measurements were performed on photomask blanks and substrates for EUV lithography. The measurement results are used to model photomask blank behavior on chucks.

- Developed a new approach to the challenge of measuring the radius of curvature of surfaces with a large radius of curvature. Examples of such surfaces are mirrors in beamlines and imaging systems, and test plates for evaluating lenses. The traditional radius bench measurement method is difficult to apply to these surfaces due to the required large displacement of the test artifact and the large cavity length. The new NIST approach eliminates these requirements through the application of a twin-Fresnel zone plate, a nano-structured optical element that incorporates two different focal lengths.

- Developed, in collaboration with NASA, a method that employs a mirror with a special height relief pattern to assess the spatial height transfer function of an interferometer and its variation over the interferometer aperture. The increasing need for measurements of complex structures with high spatial frequency content requires consideration of the height transfer function. Analytical and experimental studies were completed that characterize the height transfer function for several interferometers and operating conditions.

- Completed, in collaboration with NASA, the design and theoretical analysis of a new measurement technique that uses two Computer Generated Holograms (CGHs) to address the challenge of measuring mandrels for the fabrication of x-ray telescope mirrors.

- Developed a toolbox for designing and analyzing phase shifting algorithms for interferometry, making it possible to measure a wider range of test parts while lowering uncertainties. For example, the toolbox made it possible to address the challenge of measuring the flatness error of optical windows with near-parallel surfaces by generating a phase-shifting algorithm that is insensitive to parasitic interference from the "back"-surface. Photomask substrates are an important example of this type of optical surface.

\section{Publications}

"Computer-Generated Hologram Cavity Interferometry Test for Large X-Ray Mandrels: Design," G. Gao, J.P. Lehan, W.W. Zhang, U. Griesmann, J.A. Soons, Optical Engineering, 48 (06), 063602, 2009.

"Radius Measurement of Spherical Surfaces with Large Radii-of-Curvature Using Dual-Focus Zone Plates," Q. Wang, G. Gao, and U. Griesmann, in Optical Fabrication and Testing, OSA Technical Digest, paper OWB2, 2008.

"Measuring the Phase Transfer Function of a PhaseShifting Interferometer," J. Chu, Q. Wang, J. Lehan, U. Griesmann, and G. Gao, Proc. SPIE, Volume 7064, 2008.

\section{Contact Information}

Johannes Soons, Program Manager,

(301) 975-6474

soons@nist.gov 


\section{Metrology for Advanced Optics Projects}

\section{Nano-Structured Optics}

\section{Challenge/Problem Addressed}

T he challenge is to advance the fabrication and performance evaluation of nano-structured optical surfaces for product innovations and for application to long-standing measurement challenges.

Modern nano-fabrication technology has made possible the realization of complex structures with dimensions comparable to, or smaller than, the wavelength of light. This capacity opens up many new ways to engineer the phase and amplitude of light waves, leading to the emergence of the new class of nano-structured optics. Nanostructured optics have properties that polished optics cannot achieve. For example, a nano-structured lens can be designed to focus a light-wave at several points in space simultaneously. Nano-structured elements offer many opportunities for innovations in optics and photonics and have become a vibrant field of research. Nano-structured optics also help solve problems in the measurement of polished optical elements. A special class of nano-structured optics, computer generated holograms (CGHs), are used as auxiliary optics for the form measurement of aspheric surfaces.

A recently purchased novel massively-parallel optical lithography tool will enable us to overcome several barriers in the fabrication of demanding nano-structured optics. This innovative new tool presents a radically new opportunity for the measurement science of precision surfaces, not merely a gradual improvement. For the first time, a tool is available that can generate features with sizes down to (and below) half the wavelength of light, while having a sufficiently high write speed to pattern the large areas needed for metrology holograms in an acceptable time. In addition, it will be possible to write directly on dimensionally stable, optical quality substrates. Incorporation of this technology into the proposed research will enable NIST to overcome existing barriers to the fabrication of metrology holograms.

Our research on nano-structured optics is conducted in collaboration with customers working on leadingedge applications. For example, in collaboration with NASA we developed a new measurement technique that uses two CGHs to measure the shape and dimen- sion of non-focusing optical surfaces. An example of this type of surface is the mandrel used to form segmented mirrors for x-ray telescopes. The segments must meet tight form error specifications but also must have small dimensional errors to have the same focus. The new measurement approach addresses the shortcomings of other methods for this very challenging measurement problem.

\section{Objectives}

- Develop methods for the performance evaluation of nano-structured optics, especially for optics with small feature sizes.

- Characterize the effect of fabrication errors on the performance of nano-structured optics and improve fabrication techniques.

- Improve methods and uncertainty statements for the application of nano-structured optics to solve longstanding challenges in the calibration of high-performance free-form optical surfaces.

\section{Accomplishments}

- Completed, in collaboration with NASA, the design and theoretical analysis of a new measurement technique that uses two $\mathrm{CGH}$ s to measure mandrels for the fabrication of $\mathrm{x}$-ray telescope mirrors. Completed the measurement setup and design of the CGHs. Developed unique fiducialization scheme to align the CGHs and fix the absolute scale of the measurement. Completed theoretical analysis of measurement uncertainties. Designed an optical test, using Hartmann screens, for the initial performance evaluation of the CGHs. Conducted process characterization experiments for fabricating the diffractive optical elements.

- Developed a new approach, using a nano-structured optical element, to the challenge of measuring the radius of curvature of surfaces with a large radius of curvature. Examples of such surfaces are mirrors in beamlines and imaging systems, and test plates for evaluating lenses. The traditional radius bench measurement method is difficult to apply to these surfaces due to the required large displacement of the test artifact and the large cavity length. The new NIST approach eliminates these requirements through the application of a twin-Fresnel zone plate, a nano-structured optical element that incorporates two different focal lengths. 


\section{Planned Future Accomplishments}

- Experimentally validate the CGH-based method to measure the form and dimension of a mandrel for shaping segmented mirrors for the International X-ray Observatory (IXO) telescope.

- Experimentally and theoretically evaluate the uncertainty and application range of the new method to measure the radius of curvature of surfaces with a large radius of curvature.

- Develop procedures for the design, fabrication, validation, and application of CGHs for the measurement of polished optical surfaces with complex shapes. Develop capability for the fabrication and validation of high-performance metrology CGHs.

\section{Customers and Collaborators}

NASA Goddard Space Flight Center; University of Stuttgart, Institute for Technical Optics; University of Arizona, College of Optical Sciences

\section{Aspheric Optics}

\section{Challenge/Problem Addressed}

- he goal of this project is to characterize and improve generic methods for the measurement of aspheric surfaces, i.e., surfaces that are neither flat nor spherical. Aspheric surfaces are indispensable in modern optical systems through their combination of high optical performance, low system weight, and low cost. However, measuring aspheric surfaces poses formidable metrology problems because of the difficulty of obtaining a reference wave-front that closely matches the desired form of the asphere. Several methods have been proposed and applied to measure aspheres, such as sub-aperture stitching, measurement of local curvature, and computer generated holograms. However, the application range and achievable uncertainties of these methods are poorly understood. This is a measurement barrier to the development, manufacture, and application of aspheric optics.

\section{Objective}

Characterize and improve the uncertainty and application range of generic methods for measuring aspheric surfaces, with a focus on sub-aperture stitching, local curvature measurement, and computer generated holograms (CGHs).

\section{Accomplishments}

- Developed methods for the uncertainty evaluation of asphere measurements obtained with stitching and local curvature metrology.

- Completed the design and fabrication of the Small Aperture Digital Interferometer (SADI), a NIST developed experimental interferometric sensor that improves the range and uncertainty of local curvature measurements.

\section{Planned Future Accomplishments}

- Complete performance evaluation of the SADI sensor for local curvature measurements.

- Develop generic methods for the uncertainty evaluation of asphere measurements obtained with CGHs.

\section{Customers and Collaborators}

Argonne National Laboratory, Advanced Photon Source; NASA Goddard Space Flight Center 


\section{Optical Metrology for the Semiconductor Industry}

\section{Challenge/Problem Addressed}

$T_{\text {den }}^{\text {he }}$ he semiconductor industry expects continued demand for improved wafer flatness at the exposure site to avoid blurring of ever smaller circuit features due to out-of-focus exposures. The International Roadmap for the Semiconductor Industry (ITRS) predicts an allowed site flatness error for $300 \mathrm{~mm}$ wafers of less than $42 \mathrm{~nm}$ by 2010, and $23 \mathrm{~nm}$ for $450 \mathrm{~mm}$ wafers by 2015 . This is a challenge for both wafer polishing and wafer metrology tools. In addition, next-generation extreme ultraviolet lithography (EUVL) has strict flatness requirements for the photomasks to avoid aberrations of the projected circuit features.

\section{Objective}

D rovide measurement methods and reference artifacts that address future requirements of the semiconductor industry in the areas of silicon wafer flatness and extreme ultraviolet lithography

\section{Accomplishments}

- Developed capability for the measurement of nextgeneration $450 \mathrm{~mm}$ wafers with the $300 \mathrm{~mm}$ NIST infrared interferometer through stitching.

- Developed a new method for the deformation-free flatness measurement of EUVL photomask blanks and substrates, and provided photomask measurements to SEMATECH for modeling photomask blank behavior on chucks.

\section{Planned Future Accomplishments}

- Complete the upgrade of the phase-shifting range and algorithms of the NIST infrared interferometer to enable measurement of thin wafers $(250 \mu \mathrm{m})$ and to reduce measurement errors due to ghost reflections and non-linearities in phase shifting.

- Validate the recently developed method for the separate measurement of wafer thickness variation and refractive index variation, yielding lower uncertainties in thickness variation measurements.

\section{Customers and Collaborators}

Intel; MEMC Electronic Materials Inc.; QED

Technologies; SEMATECH; Wavefront Sciences

\section{Standards and Calibrations}

\section{Challenge/Problem Addressed}

ontroversies on specification compliance, mea-
surement procedures, and traceability continue to hamper international commerce in advanced optical elements. Uncertainty statements that comply with the GUM, the Guide to Uncertainty in Measurement, are rare in the optics industry. Companies increasingly need to adopt ISO-certified quality procedures, especially for goods that are exported. Having traceable in-house metrology with well-documented uncertainty statements is thus becoming essential.

Phase-shifting interferometry with computer-aided data analysis is the leading method for measurement of ultraprecision surfaces and optical elements. The measurement uncertainty is to a large extent determined by the form errors of the reference surfaces. Optical flats and spheres are used for this purpose, and are critical components in the traceability chain for measurements of optical elements. The need for measurements of surfaces with high spatial frequency content is increasing, which also requires consideration of the spatial height transfer function of the interferometer.

\section{Objectives}

- Provide measurement methods and services for flatness, sphericity, and radius of curvature of optical reference surfaces used for interferometric measurements of precision surfaces and optical elements. Where possible, eliminate the requirement of calibrated transfer artifacts through the development of absolute calibration methods.

- Develop uncertainty statements, compliant with the GUM, for typical measurement set-ups.

- Ensure effective access to international markets by the U.S. optics industry through international comparisons and harmonized standards for optical figure and wavefront. 


\section{Accomplishments}

- Organized a preliminary international comparison of flatness measurement capability of $300 \mathrm{~mm}$ diameter optical flats. Designed the comparison protocol, reference artifact, fixturing, and instrumented shipping container.

- Contributed to the ANSI Optics and Electro-Optics Standards Council Technical Advisory Group to improve standards developed by the ISO/TC172 Technical Committee on Optics and Photonics.

- Provided measurement services (NIST special tests) for flatness and sphericity of optical reference surfaces.

- Developed, in collaboration with NASA, a method that employs a mirror with a special height relief pattern to assess the spatial height transfer function of an interferometer and its variation over the interferometer aperture. Developed fabrication procedures for the mirror and completed analytical and experimental studies on the characteristics of the height transfer function for several interferometers and operating conditions.

- Developed a toolbox for designing and analyzing phase shifting algorithms for interferometry, making it possible to measure a wider range of test parts while lowering uncertainties. For example, the toolbox made it possible to address the challenge of measuring the flatness error of optical windows with near-parallel surfaces by generating a phase-shifting algorithm that is insensitive to parasitic interference from the "back"-surface. Photomask substrates are an important example of this type of optical surface.

\section{Planned Future Accomplishments}

- Complete an international comparison of flatness measurement capability of $300 \mathrm{~mm}$ diameter optical flats, with NIST acting as the pilot lab. The NMIs planning to participate are: CSIRO (Australia), NPL (UK), NMIJ (Japan), KRISS (Republic of Korea), and PTB (Germany).

- Develop a service for full-area calibration of optical reference surfaces, bridging the gap between recent advances achieved at NIST on the calibration of optical reference surfaces and the efficient delivery of an economically viable measurement service to customers.

- Develop the capability for full-area calibration of optical flats in a horizontal orientation.

\section{Customers and Collaborators}

Goodrich; ITT Space Systems Division; L-3 Communications; NASA; Raytheon; Zeiss IMT; Zygo 


\section{Measurements and Standards for Science-Based Manufacturing}

To remain competitive in the global marketplace, U.S. manufacturing is currently implementing fundamental changes, focused on designing and building complex, highly-customized, high quality goods -- all built speedily to meet the rapidly changing demands of the market. Manufacturers must shorten product development cycles and increase flexibility and speed of production systems and supply networks. They must do all this while also reducing environmental impacts and energy requirements. These changes require a transformation from manufacturing practices based on human experience towards scientific-based modeling, decision making, and production. This program develops fundamental measurements, standards, and tools to enable U.S. manufacturers to make this transformation.

\section{Staff}

11 NIST staff

1 NRC post-doctoral researcher

2 guest researchers

14 total FTEs

\section{Challenge}

U. S. manufacturing is undergoing fundamental changes in response to global economic and technological forces. Industry trends are shaping a new future for U.S. manufacturers - one where high-value, knowledge-intensive, highly-customized products and processes will be the new cornerstones for growth and prosperity. To remain competitive and promote growth, manufacturers must adapt to new market challenges that require more complex and individually customized products with improved quality, functionality, and performance. Furthermore, rapidly changing market demands require shorter innovation cycles, more flexible and rapidly reconfigurable manufacturing systems, integrated and streamlined communications and supply networks, reduced environmental impacts, and improved energy efficiencies. In the future, U.S. manufacturing processes must be more accurate, flexible, automated, intelligent, interoperable, reconfigurable, and sustainable. For this vision to come about, an underlying measurements and standards foundation and infrastructure must be developed and implemented so that U.S. manufacturers can quickly capitalize on future gamechanging opportunities and technological innovations. This program aims to provide competitive advantage and stimulate innovation for U.S. manufacturers by developing the metrology and standards infrastructure necessary for a science-based approach to the manufacture of complex, high-value, knowledge-intensive products.

\section{Technical Strategies}

his MEL program builds upon NIST expertise and

MEL core competencies in measurements and standards for manufacturing systems, processes, and equipment, along with substantial external partnerships with industry, academia, and other government agencies, to respond to the drivers and trends outlined above for the future of U.S. manufacturing, as well as to the critical measurement needs identified in the NIST Assessment of the U.S. Measurement System (USMS). The program addresses a science-based approach to develop the fundamental metrology and standards infrastructure necessary for U.S. manufacturers - established industries (e.g., aerospace and defense) as well as growth industries (e.g., medical devices, pharmaceuticals, alternative energy, communications, and sensors) - to overcome key technical barriers to manufacturing innovation. Results of this program will help position U.S. manufacturers to remain competitive and to achieve the future vision 
of U.S. manufacturing. The fundamental metrology and standards developed by this program will be relevant to a wide variety of new and emerging manufacturing processes and equipment, including multi-function and reconfigurable systems for fabrication, additive manufacturing systems, intelligent assembly systems, and meso/ micro-scale fabrication systems important for the medical devices industry. The objectives of this program are:

1.Develop advanced process metrology methods and tools to increase scientific understanding of manufacturing processes, including metal-based additive processes, to enable cost-effective production and decision making in response to changing conditions in the manufacturing environment.

2. Develop fundamental metrology, standards, and performance metrics for accurate complex 5 -axis motion of manufacturing equipment enabling the production of complex high-value products.

3. Develop metrology methods, standards, and performance metrics to achieve accurate, real-time, onmachine (in-situ) part measurement and certification, as well as process monitoring and control using wireless sensor networks.

\section{Results and Impacts}

- Completed comprehensive literature study on metalbased additive processes and interacted with system users to identify the current state-of-the-art and challenges. Evaluated typical part errors and use of test parts as the basis for new standards to characterize the performance of additive manufacturing systems.

- Contributed to two milestone events for advancing additive manufacturing capabilities: initiation of firstever standards activities for additive manufacturing through the newly-formed ASTM F42 (Additive Manufacturing Technologies) committee and development of a roadmap with industry experts to set priorities and guide additive manufacturing research over the next 10-12 years.

- Demonstrated the successful operation of a newly developed in-situ measurement system for cutting tool dynamics ensuring efficient stable machining processes, greatly reducing time and expertise necessary to conduct such measurements by machine operators on the shop floor.

- Reduced the measurement uncertainty of infrared micro-videography for measuring the temperature distribution of the cutting zone during orthogonal cutting, enabling temperature measurement of small features such as the shear zone. Results are used to improve predictive models of machining processes.

- Completed characterization of material properties of titanium alloy at cutting conditions. The experimental data is used to develop a science-based understanding of differences in machineability.

- Conducted, in collaboration with Kennametal, highspeed micro-videography experiments for the effects of tool insert geometry on material flow and other phenomena in the cutting zone to aid development of new cutting tools.

- Contributed towards the first-ever ISO draft standards in two critical areas that respond to industry needs for high-precision complex products:

- Standard test methods for evaluating the accuracy of 5-axis complex motion for a new generation of 4- and 5-axis machine tools (ISO/CD 10791-6, Test Conditions for Machine Centers),

- Standard test methods for evaluating on-machine measuring performance of machine tools using touch-trigger probes (ISO/DIS 230-10, Test Code for Machine Tools).

\section{Selected Publications}

"Development of a metrology frame to improve the positioning accuracy of micro/meso-scale machine tools," S.P. Moylan, D. Hong, B.N. Damazo, J.A. Soons, and M.A. Donmez, International Journal of Mechatronics and Manufacturing Systems, Vol. 2, No. 5/6, 2009.

"High-Speed Microvideography Observations of the Periodic Catastrophic Shear Event in Cutting AISI 1045 Steel," J.C. Heigel and E.P. Whitenton, Transactions of NAMRI/SME, Vol. 37, 2009. (Best Paper Award)

"Characterization of Uncertainties when Measuring Metal Cutting Temperatures Using Infrared Radiation Thermography," E.P. Whitenton, Proceedings of SPIE Volume 7299, Thermosense XXXI, 2009.

\section{Contact Information}

Alkan Donmez, Program Manager

(301) 975-6618

alkan.donmex@nist.gov 
Measurements and Standards for Science-Based Manufacturing Projects

\section{Fundamental Metrology for Material Processing}

\section{Challenge/Problem Addressed}

A anufacturing of high-value, knowledge-intensive $\checkmark$ products requires timely and accurate knowledge about the manufacturing process and the condition of the equipment, process, and part. Knowledge-intensive manufacturing processes with advanced capability and functionality will be the primary enabler for U.S. companies to cost-effectively manufacture complex and difficult-to-make products. For most manufacturing situations, this process knowledge is largely non-existent, incomplete, or non-quantified. There is currently insufficient science-based understanding of manufacturing processes to achieve the desired knowledge-intensive products and processes in a cost-efficient and highly productive manner. Fundamental metrology methods and standards are needed for real-time manufacturing process monitoring and control and for characterizing the key process phenomena necessary for making informed manufacturing decisions in response to changing conditions in the manufacturing environment. In addition, once timely and accurate process knowledge is obtained, further methods and standards are needed for the unambiguous representation and communication of such knowledge and the integration of information from diverse sources.

\section{Objectives}

D evelop the advanced process metrology methods and tools to increase scientific understanding of existing, as well as new and emerging manufacturing processes and equipment, including multi-function and reconfigurable systems for fabrication, intelligent assembly systems, and meso/micro-scale fabrication systems.
Determine specific application case studies as appropriate based on industry interactions and priorities. Several process parameters and phenomena are expected to be of critical importance for multiple manufacturing processes, such that common and generic process metrology approaches can be devised. These process phenomena include forces, temperatures, and material transformations at the material/tool interface, tool wear and performance, friction considerations, system vibrations and dynamic response, bulk material properties, and waste by-products of the manufacturing process.

Obtain enhanced information related to manufacturing processes and equipment through the new measurement methods and from in-situ and real-time sensing for process validation. Standardized approaches for sharing process knowledge among the various manufacturing system components will be used for intelligent, real-time decision-making. Generic, physics-based measurement methods will be developed, promoted through national and international standards organizations, and transferred to industry to increase scientific understanding of manufacturing systems, processes, and equipment.

\section{Accomplishments}

- Applied the NIST pulse-heated Kolsky bar to characterize material properties at conditions relevant to machining (high strain rates and rapid heating) for titanium and steel. Conducted instrumented orthogonal cutting experiments with high-speed videography to measure strain, strain rate, chip segmentation and contact length; infrared videography to measure the temperature distribution of chip and tool; dynamometry to measure cutting forces; and post-process chip and tool microscopy to measure chip morphology, tool wear, and adhered inclusions on the tool. Results were combined with finite element simulations of the cutting process to improve science based understanding of differences in machineability.

- Conducted, in collaboration with Kennametal, highspeed micro-videography experiments for the effects of tool insert geometry on material flow and other phenomena in the cutting zone to aid development of new cutting tools. 
- Obtained results from the NIST pulse-heated Kolsky bar tests on AISI 1075 steel that demonstrate that the unique rapid heating technique reveals constitutive material behavior that cannot be predicted by the Johnson-Cook flow stress model that is widely used for the simulation of high-speed machining processes.

- Reduced the measurement uncertainty of infrared micro-videography for measuring the temperature distribution of the cutting zone during orthogonal cutting. Characterized and reduced the significant measurement errors that can occur during segmented chip formation due to motion blur, the small size of regions with elevated temperatures, and the camera transfer function. Reduced the uncertainty of temperature measurements of tool inserts by establishing that "pre-oxidizing" reduces emissivity variations during cutting experiments. Developed a technique to measure the temperature of cutting inserts with embedded thermocouples and compared thermocouple readings with thermal images, achieving good agreement.

- Developed a technique that enables implementation of material data obtained from Kolsky bar experiments into proprietary material models used in finite element software for the analysis of machining processes. A key part of the technique is the application of the software to simulate a Kolsky bar experiment.

\section{Planned Future Accomplishments}

- Publish a guide to uncertainty assessment for infrared micro-videography of cutting processes.

- Develop new metrology devices, methods and standards for critical manufacturing process parameters (such as force, torque, power, current, temperature, etc.) to enable real-time process monitoring and control.

- Develop non-contact optical measurement capability for characterizing material strain and transformations during manufacturing processes to better understand the manufacturing process by observing its effect on the workpiece material.

\section{Customers and Collaborators}

Third Wave Systems; Kennametal; Caterpillar; Ford Motor; United Technologies; Alcoa; Los Alamos

National Laboratory 
Investigation of

Measurements and

Standards Needs for

Metal-Based

Additive Processes

\section{Challenge/Problem Addressed}

merging manufacturing technologies for producing

highly-complex customized components include metal-based additive processes, such as selective laser sintering (SLS), direct-metal laser sintering (DMLS), and laser engineered net shaping (LENS). Technologies such as these have been in development over the past decade and their capabilities have grown significantly. However, several barriers have prevented metal-based additive processes from reaching their potential. Primary barriers are insufficient part accuarcy and surface finish, lacck of standards for proccess certification, limited data formats, and low processing speed.

\section{Objectives}

nvestigate the fundamental characteristics of these new processes, address critical measurement and standards issues, and develop metrology tools to improve the science-based understanding of these processes.

Leverage existing MEL skills and expertise to impact metal-based additive manufacturing in areas such as precision motion control, equipment performance assessment and metrics, manufacturing process control and automation, measurement methods for determining process parameters such as temperatures and forces, and remote sensing and condition monitoring of system performance. The fundamental metrology and standards developed by this program will be relevant to a wide variety of new and emerging manufacturing processes and equipment.

\section{Accomplishments}

- Completed comprehensive literature study on metalbased additive processes and interacted with system users to identify the current state-of-the-art and challenges. Evaluated typical part errors and use of test parts as the basis for new standards to characterize the performance of additive manufacturing systems.

- Contributed to two milestone events for advancing additive manufacturing capabilities: initiation of firstever standards activities for additive manufacturing through the newly-formed ASTM F42 (Additive Manufacturing Technologies) committee and development of a roadmap with industry experts to set priorities and guide additive manufacturing research over the next 10-12 years.

\section{Planned Future Accomplishments}

evelop performance metrics and metrology tools to characterize metal-based additive fabrication process.

\section{Customers and Collaborators}

3D Systems; Honeywell; Boing; GE Aviation; U.S. Air Force Research lab; Medical Modeling Inc. 


\section{Metrology and Standards for Coordinated 5-axis Motion}

\section{Challenge/Problem Addressed}

T he high-value, complex (free-form) products of the future require manufacturing processes and equipment capable of generating accurate complex motion, including coordinated multi-axis linear and rotational motion. Existing measurement methods and standards focus on evaluating the single-axis motion needed for the manufacture of simple, prismatic products. Although methods are available to examine errors associated with coordinated motion of two simply-moving components, precise measurement methods for the complex motion of manufacturing equipment with five or more degrees of freedom, necessary to fabricate the complex, free-form products of the future, are currently nonexistent. The measurement problem is compounded for the manufacture of meso/micro-scale products since traditional metrology instruments are typically too large and cannot fit within the workspaces of the manufacturing systems needed at this scale. New measurement methods, systems, standards, and performance metrics are urgently needed by U.S. manufacturers to understand and apply the capabilities of the next-generation, multi-axis reconfigurable manufacturing equipment for innovative new products.

\section{Objectives}

evelop fundamental metrology, standards, and performance metrics for accurate complex motion for manufacturing equipment enabling the production of complex high-value products.

\section{Accomplishments}

- Developed a method to determine the orientation of a non-orthogonal axis of rotation of a rotary table in the machine coordinate system, providing a means to compensate for inaccuracies in the orientation and thus greatly improving the performance of 5-axis machine tools.

- In collaboration with an international working group, designed and machined a test artifact for testing 5-axis machine tools. The form measurements of the test artifact were compared to kinematic test results obtained by the telescoping ballbar to validate the method of using test artifacts to quickly evaluate 5 -axis machine performance.

- Completed experiments that compared multiple test methods, patterns, and data analyses considered for the new draft standard (ISO/CD 10791-6) for assessing the contouring performance of coordinated 5-axis motion to ensure the uniformity and consistency in the test results. The results of the study were presented to the ISO committee to modify the draft standard.

- Developed kinematic and control system models of the NIST 5-axis machine tool to predict its contouring performance. Such models will be used to interpret measurement data and for diagnostics.

- Developed solid models of the 5-axis machine structure, measuring equipment, and the fixtures to virtually assemble the measurement setup to reduce time for designing and carrying out tests for various machine structures.

\section{Planned Future Accomplishments}

- Integrate structural models, kinematic models, and servo control models to develop comprehensive performance models for machine structures.

- Finalize test artifacts and test methods using machined test pieces to be proposed for standardization.

- Develop international standard for testing 5-axis coordinated motion for manufacturing equipment.

\section{Customers and Collaborators}

Ford Motor; Lockheed Martin; BAE Systems; Caterpillar; Remmele Engineering; United Technologies; Cincinnati Machines 


\section{Ultra-Precision Linear Motion Metrology}

\section{Challenge/Problem Addressed}

A any new emerging applications of nanotech$\checkmark$ nologies require the use of small precision linear translation stages that have an exceptionally long range of motion (tens of millimeters) with sub-micrometer positioning accuracy and repeatability. A short list of these technologies may include micro/meso-scale machine tools and coordinate measuring machines, lithography equipment, and scanning probe microscopy instruments, to name a few. The need has inspired the development of many off-the-shelf turnkey linear translation systems that have travel ranges in the tens of millimeters with specified positioning accuracies on the order of tens of nanometers or better. Manufacturers and customers of such systems have begun to recognize the difficulties in certifying the accuracy and repeatability of the performance measurements of these systems with appropriate levels of uncertainty, i.e., a few nanometers or better. Sources of measurement uncertainty that were once considered insignificant when measuring in the micrometer regime are now becoming very significant while measuring in the nanometer regime.

\section{Objectives}

n collaboration with manufacturers of long-range, linear nano-translation stages, investigate and evaluate the best practices, standards, and equipment used for measuring and determining the accuracy and repeatability of positioning numerically-controlled axes and establish a low uncertainty measurement capability at NIST.

A variety of measurement uncertainty sources including, but not limited to, the measuring devices, environment, measurement setup, and the thermal behavior of the stage will be examined. Instrumentation and techniques crucial in reducing the measurement uncertainty will be evaluated. Based on the findings, the measurement facilities will be upgraded to enable significant reductions in uncertainties.

\section{Accomplishments}

This project was initiated in FY2010.

\section{Planned Future Accomplishments}

- Develop detailed uncertainty analysis and uncertainty budgets associated with the measurement methods and instrumentation used for performance measurements of linear translation stages.

- Evaluate two long-range, linear nano-translation stages.

- Develop a highly-controlled test chamber.

- Develop a measurement capability with an uncertainty better than $5 \mathrm{~nm}$.

- Publish a technical paper describing the methods to reduce the uncertainties and the results of performance measurements under these conditions.

\section{Customers and Collaborators}

Aerotech 


\section{In-situ 3D Optical and Me- chanical Metrology of Fabricated Parts}

\section{Challenge/Problem Addressed}

\begin{abstract}
key objective in durable goods manufacturing is faster throughput by eliminating the need for offline part inspection, typically performed using coordinate measuring machines (CMMs) and related inspection tools. U.S. manufacturers currently use off-line part measurement and certification for quality control of high-value products. Despite their high accuracy, CMM measurements are time-consuming and must be performed in specialized environments removed from the production floor, resulting in increased production times and higher manufacturing costs. This project will address one component of this challenge by developing on-machine (in-situ) metrology methods, standards, and performance metrics for inspecting and certifying highvalue products while the fabricated part is still fixtured within the manufacturing equipment - with a focus on the growing importance of micro/meso-scale fabrication systems and products.
\end{abstract}

The measurement problem is more complex for micro/ meso-scaled components due to difficulties in handling the small parts after their fabrication. Optical and mechanical metrology methods will be incorporated into manufacturing systems and evaluated for in-situ part certification and independent measurement of part features relevant to a variety of platforms and applications. The real-time product data obtained by the in-situ measurement systems will form the basis for future manufacturing process monitoring and control.

\section{Objectives}

D evelop measurement methods and systems to enable in-situ 3D optical and mechanical metrology for fabricated parts to obtain faster throughput by eliminating the need for off-line part inspection. Research will focus on extending the application range and accuracy of 3D imaging systems in production environments, overcoming existing limitations due to line-ofsight requirements, surface imperfections, and variability in part reflectivity, lighting, shadowing, and color.

\section{Accomplishments}

- Completed the implementation of the metrology frame on the meso-scale milling machine demonstrating over $90 \%$ improvement in machine performance.

- Completed the development and installation of fringe projection based measurement system for on-machine measurement of 3D features of meso/micro scale machined components.

- Completed the uncertainty analysis of two-dimensional measurements by the fringe projection system, using symbolic manipulations to enable the complex analysis required and highlighting the sensitivity to the initial calibration procedure.

- Developed a new Matlab code for image processing to be used with the fringe projection system to enable on-machine measurements of dimensions and form of micro-scale to meso-scale parts.

- Completed development of the in-situ automated measuring system for tool dynamics, demonstrating transparent operation without needing a dynamics expert on the shop floor and the benefits of up-to-date use of tool dynamics information in predicting stability regions.

\section{Planned Future Accomplishments}

- Reduce the uncertainty of fringe projection measurements by a factor of $30 \%$.

- In collaboration with other MEL programs, establish method to combine multiple views of the same part into a single data set without the need for registration artifacts.

- Develop method for on-machine coordinate metrology assisted by metrology frames to reduce the uncertainty of dimensional measurements by a factor of $30 \%$.

\section{Customers and Collaborators}

Atometric; Microlution; Remmele Engineering 


\section{Performance Metrics for Manufacturing Equipment Used as Measuring Tools}

\section{Challenge/Problem Addressed}

O

ne aspect of the future vision for U.S. manufactur-

ing is that product inspection and certification will occur while the fabricated part remains fixtured within the manufacturing equipment that produced the part. A primary benefit of this approach is that further processing of the part is greatly simplified if the inspection results indicate that changes are necessary. However, a substantial challenge is presented to maintain measurement accuracy when the manufacturing equipment is also used as a measuring machine. Specifically, significant measurement uncertainties can be generated if the manufacturing equipment uses the same controlled motion, axes, and algorithms to measure the part as it used to create the part. The potential exists for machine errors to create unintended deviations in the part that may go undetected in the part inspection and certification measurements.

\section{Objectives}

Develop the performance standards and standardized and their associated uncertainty budgets that are critical to achieve accurate, in-situ part inspection and certification. These standards are necessary to drive product and process innovations in sensors for manufacturing, manufacturing control systems, product fabrication systems, and metrology tools for manufacturing.

\section{Accomplishments}

- In collaboration with the international working group, developed and validated a series of tests to assess the measuring performance of machine tools using touch trigger probes. The draft standard (ISO/DIS 230-10, Test Code for Machine Tools) is currently being circulated for comments from the member bodies.

\section{Planned Future Accomplishments}

- Develop a methodology for geometry-based uncertainty budgets applicable for various on-machine measurement implementations.

- Complete the internationally-accepted ISO 230-10 standard.

\section{Customers and Collaborators}

Renishaw; General Motors; Ford Motor; Caterpillar; United Technologies; Remmele Engineering; TechSolve 


\section{Manufacturing Process Monitoring and Control Using Wireless Sensor Networks}

\section{Challenge/Problem Addressed}

ensors are indispensable in a wide variety of manu- facturing applications, ranging from equipment and environmental control, monitoring and adjustment of production processes, and quality control of finished products. The large majority of current sensor implementations in factory settings are based on wired communications, rather than wireless. In comparison to traditional wired networks, wireless sensor systems would provide advantages in the manufacturing environment, such as increased flexibility for locating and reconfiguring sensors, elimination of wires in potentially hazardous locations, and ease of network maintenance. This is particularly true for the installation of sensor network/ systems on an existing shop floor, because wireless sensors are easy and quick to apply and reconfigure, and there is no need for time-consuming and costly cable laying and maintenance. The technical challenges that now preclude widespread and effective use of wireless sensors and communications on the factory floor include (1) reliable receipt of sensor signals, (2) sufficient power for the sensor to provide the desired signal strength, useful life, and continuous communications, and (3) lack of interoperable sensor data and systems.

\section{Objectives}

Develop models and performance metrics to understand and predict response of sensors used for measurement and control of the manufacturing process. Develop a prototype wireless sensor network based on the IEEE 1451 family of smart transducer interface standards that enables us to gain better insights into the implementation of wireless sensor standards and how they can be applied to shop floor application.

\section{Accomplishments}

- Developed a model that enables analysis of signals from accelerometers used to monitor machining center manufacturing process. Analytical models are essential tools for understanding and predicting sensor response.

- Developed the hardware, software, and web-browser data viewer for a microprocessor-based wireless transducer interface module. These are essential prerequisites to the development of a prototype wireless sensor network.

- Developed and conducted tests of a preliminary wireless sensor network on the NIST shop floor for monitoring three IEEE 1451-based sensors installed on a Mazak turning center. A three-axis accelerometer and two temperature sensors provided five data channels. Data integrity was found to change significantly as the wireless transmission environment was varied.

\section{Planned Future Accomplishments}

- Develop and implement a wireless sensor network including network processor, and multiple wireless transducer interface modules and sensors based on wireless sensor standards. This enables us to gain better insights into the implementation of wireless sensor standards.

- Develop IEEE 1451-based software modules and application program interface and integrate with the wireless sensor network to demonstrate new capabilities for manufacturing equipment monitoring.

\section{Customers and Collaborators}

Agilent; Emerson; E-Sensors; Exxon Mobil; Lockheed Martin; Northrop Grumman; Open Geospatial Consortium; Raytheon; Texas Instruments; Tyson Foods 


\section{Mechanical Metrology}

Providing critical measurements of mass, force, vibration, and acoustics for a broad range of industries and aspects of everyday life. Such measurements establish uniformity and reliability for countless products and applications, such as grocery store scales, microphones, pharmaceuticals, and the structural integrity of bridges. The program's wide-ranging goals include participating in international efforts to redefine the Kilogram, establishing new molecular force standards, improving timeliness and performance of current calibration services, and developing next-generation measurements for acoustics and vibration. Such services help provide industry with the tools needed to maintain or establish the highest levels of competitiveness in world markets.

\section{Staff}

18 NIST staff

1 NRC post-doctoral researcher

2 guest researchers

21 total FTEs

\section{Challenge}

dvance the science of International System of Units (SI) mechanical metrology to anticipate the future needs - and address unmet present needs - of U.S. industry and government in the areas of acoustics, force, mass, and vibration. New and innovative approaches that push the limits of the current state-of-the-art are required to establish the future measurements and standards needed for continued advancement and competitiveness of critical U.S. industries in the global market.

\section{Technical Strategies}

echanical metrology plays a critical role in nearly $\checkmark$ all sectors of the U.S. economy and in everyday life. Industry needs for measurement traceability, compliance with regulatory requirements, harmonized standards for international trade, and improved capabilities to cost-effectively manufacture higher quality and more complex products require advancements in traceable measurements for the mechanical metrology areas of acoustics, force, mass, and vibration.
MEL provides industry and government with measurement services and research for next-generation measurement capabilities, ensuring delivery of state-of-the-art measurement accuracy for high-impact SI mechanical metrology. Program priorities are identified and established based on ongoing interactions with industry and other partners. This customer input is used to direct the program technical agenda for current and future measurements, services, and standards.

For each MEL mechanical metrology area, the program contains the following elements:

- Research and Development

- Measurement Services

- Quality System Development and Conformance

- Leadership in National and International Standards

- International Comparisons of Measurement Capability To ensure the competitiveness of U.S. industry in the world marketplace, the program maintains active participation in corresponding CIPM (International Committee for Weights and Measures) consultative committees and SIM (Interamerican Metrology System) metrology working groups. The program fulfills NIST's role as a signatory of the Mutual Recognition Arrangement (MRA) for measurement capabilities in the areas of mass, force, acoustics, and vibration. Measurement methods and uncertainty evaluation techniques developed by MEL are transferred to U.S. industry and other government agencies through participation in national and international standards activities. The program provides state-of-theart measurement services in compliance with the ISO 
17025 quality system standard to provide NIST customers with traceable calibrations at the top of the SI traceability chain.

A primary program objective is to conduct cutting-edge research and development to anticipate and drive future advancements of U.S. industry in all four mechanical metrology areas. Research activities ensure that MEL will meet increasing U.S. industry and government demands for higher-accuracy, more cost-effective mechanical measurements by: (1) developing next-generation measurements and calibration systems with lower measurement uncertainties and expanded measurement ranges; and (2) developing innovative new approaches to measuring mechanical quantities.

\section{Results and Impacts}

- Completed crucial steps towards a practical method for realization of a new kilogram definition, including development of a stable suspension technique based on magnetic field sensing and servo control, analysis of the effect of magnetic pole shapes on suspension and stability, and stable levitation (less than $1 \mathrm{mg}$ ) of $200 \mathrm{~g}$ to $1 \mathrm{~kg}$ mass artifacts using the new suspension. This will provide the link between the existing artifact-based definition realized in air and the future realization of mass based on fundamental constants in vacuum. This link is essential to guarantee continuity and equivalency of mass measurements.

- Demonstrated first accurate scheme for practically realizing forces below a nanonewton enabling direct calibration of probes used in single molecule mechanical characterization experiments.

- Developed reliable methods for performing complex single DNA molecule experiments and achieved the first traceable measurements of this force, therefore demonstrating the use of biological molecules as intrinsic force standards.

- Demonstrated stable creation and manipulation of single atom chains using a new instrument developed as part of the intrinsic force project. As part of this instrument development, also created a fiber interferometer system for the accurate measurement of picoscale displacements.
- Completed the development of new measurement system, software, and procedures for reciprocity calibrations of laboratory standard microphones from $31.5 \mathrm{~Hz}$ to $20 \mathrm{kHz}$ in plane-wave air-filled couplers. This allows NIST to provide next-generation acoustical measurement services that halves our measurement uncertainties in the most critical frequency range.

- Completed analysis and final reports of critical key comparisons in the areas of force and vibration metrology where NIST served as pilot laboratory. This guarantees the recognition and validity of the calibration and measurement capabilities of the participating countries under the mutual recognition arrangement (MRA), thereby supporting international trade.

- Provided critical and state-of-the-art measurement services to the U.S. government and industry through nearly 2000 tests performed annually for over 100 customers.

\section{Key Publications}

Jabbour, Z.J., Abbott, P., Williams, E., Liu, R., Lee, V., "Linking air and vacuum mass measurement by magnetic levitation," Metrologia, 46, 339 (2009).

Smith, Pratt, and Howard, "A fiber-optic interferometer with subpicometer resolution for dc and low-frequency displacement measurement," Rev. Sci. Instr., 80, 035105 (2009).

Evans, D.J.; Hornikova, A.; Leigh, S.D.; Rukhin, A.L.; Strawderman, W. "Report on Acceleration Comparison SIM.AUV.V-K1," Metrologia, 46, Tech. Suppl., 09003 (2009).

\section{Contact Information}

Zeina Jabbour, Program Manager, (301) 975-4468

zeina.jabbour@nist.gov 
Mechanical Metrology Projects

Redefinition of the

Kilogram: Air-to-Vacuum Mass Comparison

\section{Challenge/Problem Addressed}

xperiments to redefine the kilogram with an invari-

ant of nature (such as the Watt balance or the Avogadro project) are performed in vacuum, while current realization of the mass unit and all associated methods of dissemination of the unit are done in air. Transfer of the unit to vacuum requires an unbroken traceability chain to the international prototype kilogram, as well as characterization of the stability of the artifacts and their surfaces during transfers from air to vacuum and vice versa. This requirement remains a major challenge and obstacle for further advances in redefining the unit of mass.

\section{Objectives}

stablish the first complete

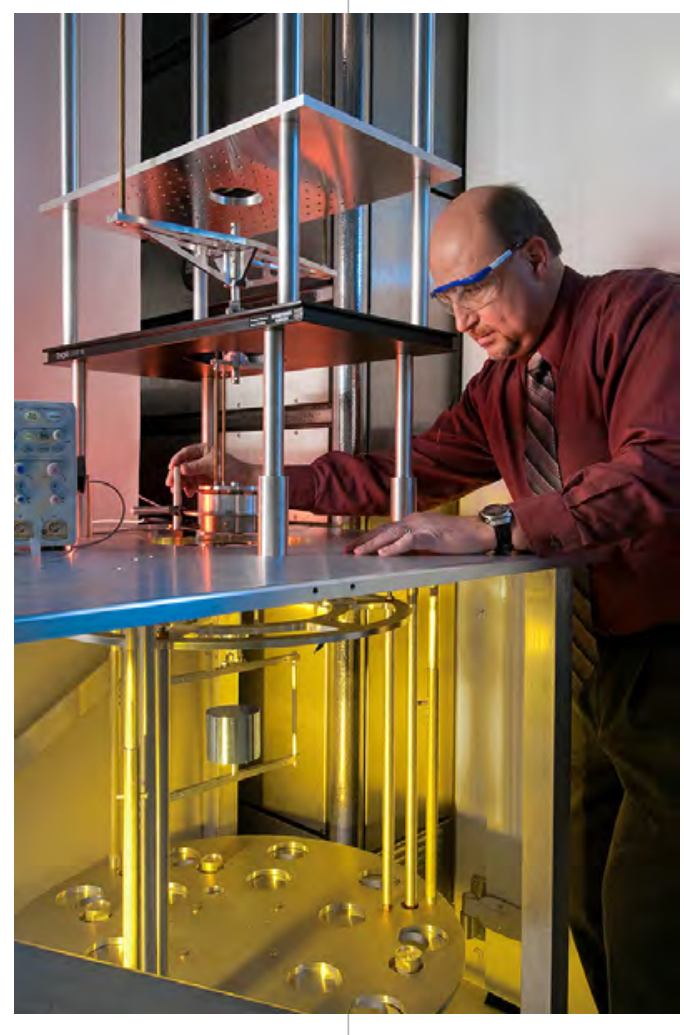

- Completed long-term (over 18 months) study of several diamond-like carbon (DLC) coated stainless steel $1 \mathrm{~kg}$ mass artifacts for mass stability. Several coating processes are being considered to achieve chemical inertness, wear resistance, and overall stability in the dissemination of the unit of mass.

\section{Planned Future Accomplishments}

- Incorporate new suspension system into non-magnetic vessel and interface with sensitive mass balance and make first direct air-to-vacuum mass comparison using magnetic suspension technique with $1 \mathrm{~kg}$ mass artifacts.

- Identify sources of measuresemination system that directly ties the current air-based kilogram definition to the vacuum-based alternative definition using stable artifacts with wear-resistant and chemical inertness characteristics that are maintained in controlled environments. The realization apparatus will use magnetic suspension to couple a mass artifact in the air chamber to a sensitive balance in the vacuum chamber. In this way, the artifact in air can be compared to a stable artifact traceable to the new definition of the kilogram in the vacuum chamber.

\section{Accomplishments}

- Completed the design and in-house construction of the non-magnetic aluminum vessel accommodating separate air and vacuum chambers. Incorporated appropriate vacuum technology (pumps, gauges, and components), vacuum and leak testing, and ment uncertainty in magnetic suspension technique and estimate their magnitudes. Refine experimental technique to have combined relative standard uncertainty of $2 \times 10^{-8}$ or lower.

- Based on long term study results, identify suitable coating for use in making stable mass artifacts.

- Propose magnetic suspension air-to-vacuum mass comparison technique as a Mise en Pratique (practical method for realization) for traceability to and dissemination of new kilogram definition.

\section{Customers and Collaborators}

U.S. State Weights \& Measures Laboratories; National Metrology Institutes; fundamental constants scientific community; Argonne National Laboratory; SilcoTech 


\section{Small Force Metrology}

\section{Challenge/Problem Addressed}

Thestar he challenge is to provide accurate references (standards and techniques) for the calibration of forces measured using instrumented indentation equipment, optical and magnetic tweezers, surface forces apparatuses, and interfacial and atomic force microscopes. The forces measured by these instruments range from piconewtons (the light pressure exerted by a typical laser pointer) in singlemolecule biophysical studies of disease therapies, to tens of millinewtons (the weight corresponding to a gram mass) in studies of new dielectric coatings for metals and semiconductors.

No accurate standards or reference forces exist below 10 micronewtons for the calibrations of these various scientific instruments.

The major push for accurate force measurement across the growing range of nanotechnological characterization tools is twofold. First, accuracy is required to reconcile theory with experiment. The leading wave of nanomanufactured products is composed primarily of new, nanostructured materials. The manufacturers creating these materials (most notably large U.S. multi-national companies focused on polymer coatings) cannot adequately characterize the performance of these new nanoengineered materials using the existing state of nanoscale materials characterization tools, and must instead rely on costly functional testing. Second, instrument providers and users demand a common level playing field to resolve inconsistencies between various manufacturers' claims in an increasingly competitive nanotechnology market.

More and more instrument users are lobbying for ready access to cheap, reliable force standards, even taking the initiative to work with instrument manufacturers to develop ASTM and ISO standards. Although mechanical characterization is widely used in nanotechnology for industrial as well as scientific applications, the forces applied by atomic force microscopes do not have SI traceability over the commonly used force range from piconewtons $\left(10^{-12} \mathrm{~N}\right)$ to nanonewtons $\left(10^{-9} \mathrm{~N}\right)$. Current generation $\mathrm{SI}$ traceability relies on a technologically challenging force transfer in a NIST-operated electrostatic force balance that provides a micronewton $\left(10^{-6} \mathrm{~N}\right)$ force calibration with nanonewton sensitivity.

\section{Objectives}

- Create the means to achieve SI traceability for small force measurement and instrumentation, including development and testing of internationallyaccepted standards of force in the regime below $10^{-3} \mathrm{~N}$, exploration of atomic and single-molecule forces as potential intrinsic standards and refer-

ences, and development of new instrument platforms at NIST that are capable of realizing ever smaller values of force in terms of traceably realized SI base units and quantum invariants. 
- Develop or improve upon techniques for the dissemination of small force using microcantilevers to support nanomechanical investigators who are using atomic force microscopy to probe the physical characteristics of matter at the nanoscale.

- Lead and participate in international comparisons of small force calibration and the related topic of cantilever stiffness calibration with the objective of representing U.S. industrial and scientific interests as new international standards are proposed and vetted.

\section{Accomplishments}

- Demonstrated the first accurate scheme for practically realizing forces below the level of a nanonewton enabling the direct calibration of probes used in single molecule mechanical characterization experiments. The technique, which exploits a calculable electrostatic force between a flat probe tip and a thirty micrometer diameter metallic sphere, has been used as the basis for calibrating both normal and lateral force sensitivities of colloidal probe AFM instruments used at NIST.

- Demonstrated that biological molecules could serve as intrinsic force standards. Biological molecules, in general, and DNA, in particular, exhibit some unique properties that make them excellent candidates for future NIST reference materials. In 2009, reliable methods for performing this complex single molecule experiment were developed and the first traceable measurements of this force were achieved.

- Demonstrated the stable creation and manipulation of single atom chains using a newly-developed instrument for the feedback-stabilized break junction experiment. To achieve this result, we created a fiber interferometer system for the accurate measurement of picoscale displacements.

- Demonstrated a new method for characterizing the sliding friction between an atomic force microscope (AFM) cantilever tip and a compliant surface. The new technique yields the coefficient of friction between the surface and tip and also allows us to correct the results of cantilever-on-cantilever calibrations of AFM sensitivities. The results greatly improve the calibration accuracy (by as much as $20 \%$ in some instances).
- Completed an informal round robin study of small force calibrations with Korea, Germany, and England that was initiated in 2008. This test is a first step in verification that micronewton force instruments and associated practices for dissemination of the micronewton are in agreement among the NMI laboratories that support such measurements. This comparison should also underpin the new ISO standard on cantilever stiffness calibration.

\section{Planned Future Accomplishments}

- Complete development of the new platform for accurate single molecule tensile testing to achieve a stable, traceable system for calibrating intrinsic force standards based on molecular interactions.

- Validate the accuracy of dynamic calibration techniques that use laser vibrometers to measure AFM cantilever thermal response and establish accurate correction coefficients to enable accurate, highthroughput calibration of AFM cantilever stiffness.

- Implement photon momentum to apply calculable dynamic forces directly to a force sensor to obtain accurate optical forces.

\section{Customers and Collaborators}

Asylum Research; Veeco Products 


\section{Next-Generation Rectilinear Wide-Bandwidth Vibration Measurements}

\section{Challenge/Problem Addressed}

$\mathrm{v}$ ibration measurements are used for environmental

testing, diagnostics, product development, condition monitoring, process control, servo sensors, and global positioning. Some applications involve very high volume products or very expensive products. For example, the automotive industry relies heavily on absolute measurements of acceleration involving hundreds of transducers per test, and many tens of staff years are spent measuring accelerations on vehicles for airbag-deployment systems and for ride control of specific vehicle classes. Delivery of such systems in vehicles is measured in the millions of units. Other vibration metrology applications include the development and qualification of products of very high cost, such as aircraft and their components.

NIST currently provides vibration calibration services for a broad range of industries and government agencies using four aging accelerometer calibration systems developed in early 1960s. NIST customers, especially in the automotive and aerospace industries, need lower uncertainty in vibration calibrations due to increased global competitive pressures and safety requirements. Customer requirements drive the need for a costeffective, operationally efficient, and reliable system to calibrate the magnitude and phase sensitivity of accelerometers with reduced uncertainty and turn-around time. The performance limitations and maintenance needs of the current calibration systems create significant bottlenecks in timely and accurate NIST response to customer requirements.

\section{Objective:}

D evelop and implement a next-generation calibration system for measurement of dynamic acceleration that would operate over the extended frequency range of $5 \mathrm{~Hz}$ to $20 \mathrm{kHz}$ (covering the majority of current vibration measurement service requests) and replace four aging calibration systems used in five NIST SP250 measurement services. The new system will also provide a new capability to calibrate the phase of accelerometers - particularly important for accelerometers used for modal analysis and measurement of transient events (e.g., shock).

\section{Accomplishments}

- Developed testbed for the evaluation of shakers consisting of a vibration isolated table, instrumentation, interferometer and optics, and a vibrometer. This testbed allows for easy setup of shakers for evaluation in typical operational configurations.

- Characterized performance of a commercial shaker, including characteristics such as harmonic distortion, cross-axis (transverse) motion, magnetic field strength, temperature, and uniformity of motion of the table of the moving element.

- Collaborated with the Air Force Primary Standards Lab to establish specifications, test methods, and targets for shaker performance. The Air Force has expressed interest in the results of our shaker evaluations.

\section{Planned Future Accomplishments}

- Identify commercial vibration generator that matches NIST specifications as closely as possible. Model, design, and implement any modifications required for the commercial vibration generator to meet the specified criteria.

- Develop, test, and validate SI-traceable measurement procedures using the new vibration source and establish the uncertainty budget for the new system.

\section{Customers and Collaborators}

Air Force Primary Standards Lab 


\section{Advanced Acoustical Measurements: Infrasound Metrology}

\section{Challenge/Problem Addressed}

he challenge is the development of first-ever SI-
traceable acoustical measurement capabilities and services in the infrasonic regime (from $2 \mathrm{~Hz}$ to $20 \mathrm{~Hz}$ ) to provide accuracy, traceability, and credibility in infrasonic measurements to meet industry and governmental needs. These needs are driven by the desire to study, detect, and warn of many important natural phenomena and man-made events. Natural phenomena include volcanic activity, hurricanes, tornadoes, landslides, earthquakes, meteors, atmospheric turbulence, and oceanic waves. Man-made events, such as rocket launches, airbag deployment, supersonic aircraft travel, explosions resulting from weapons tests, and tactical military operations involving vehicles and weapons also generate high-amplitude, low-frequency infrasound of great significance. Infrasonic measurements are important to the transportation industry, medical community, geophysical-science community, meteorologists, and U.S. government agencies responsible for supporting the International Monitoring System (IMS) as part of the U.S. involvement in the Comprehensive Nuclear-Test-Ban Treaty, conducting atmospheric and climate studies, developing weather prediction models (e.g., NOAA), and designing new applications for the military and national defense. Manufacturers of component infrasound transducers and systems also need the new NIST measurement capabilities and service for recognized, validated SI traceability. Measurements of hazardous sounds with high-amplitude infrasonic components, such as those produced by automotive airbag deployment, would also be provided with improved accuracy, traceability, and credibility. In particular, the Society of Automotive Engineers (SAE) requires a more accurate calibration method and standard for measuring infrasound to influence the design and operation of automotive airbags and to protect the hearing of vehicle occupants during airbag deployment.

\section{Objectives}

D evelop new acoustical measurement capabilities and a service in the infrasonic regime (from $2 \mathrm{~Hz}$ to $20 \mathrm{~Hz}$ ) to provide accuracy, traceability, and credibility in infrasonic measurements to meet industry and governmental needs.

\section{Accomplishments}

- Completed component selection and established the basic reciprocity-based measurement system design using a high-order bandpass filter set, signal generator, signal conditioning/switching apparatus, voltmeter, temperature probe, barometer, and personal computer as controller of these components using software developed by the project team.

- Completed the assembly and integration of these components, the optimization of equipment power and signal interfaces, the development of a sequence of measurement steps in the initial procedure involving the pressure-field reciprocity method and air-filled plane-wave acoustical couplers, and the development of control and data analysis software for the initial version of the system.

- Developed software to calculate microphone sensitivities from data measured with the system, in accord with equations based on reciprocity and the use of air-filled plane-wave couplers consistent with ANSI S1.15-2 and IEC 61094-2 published standards.

\section{Planned Future Accomplishments}

- Perform preliminary measurements with laboratory standard microphones and select system configuration parameters for proof tests of system.

- Perform proof tests of system using laboratory standard microphones and complete the evaluation and documentation of this system, software, and procedures.

- Develop quality system documentation and transition the new capabilities to a measurement service.

\section{Customers and Collaborators}

U.S. Department of Energy; Sandia National Laboratory; Los Alamos National Laboratory; U.S. Department of Defense; U.S. Army; NOAA; National Weather Service; Chapparel Physics 


\section{Uniform Realization of the Unit of Torque in the U.S.}

\section{Challenge/Problem Addressed}

T orque measurements are used heavily during prod-

uct assembly and testing throughout the aerospace, automotive, and manufacturing sectors. Uncertainty evaluations surrounding the measurements are based upon current best efforts by private and independent organizations. The lack of a singular national torque standards laboratory in the U.S. requires time-consuming and costly maneuvers by each of multiple individual users throughout the supply chain to justify their own systems to even a basic level of uncertainty acceptance by other users. For example recalling a single jet engine due to any problem including poor assembly of torqued components can trigger costs to the manufacturer that begin at $\$ 500,000$. The human and capital costs can be far higher when improper torque values are not identified and component failure occurs.

Various U.S. manufacturers and commercial secondary standards laboratories, including Boeing Aircraft, Instron Corporation, Interface Inc, HBM Inc, and Tovey Engineering, have either endorsed an ASTM request for NIST to conduct SI-traceable torque calibrations or have solicited NIST directly with the same request. The industry claim is that NIST involvement is needed to provide absolute measurements that will solve the existing disagreement among current laboratory calibration results. The ASTM Mechanical Testing committee has submitted a formal request to NIST as part of their effort to write and place into use several U.S. torque documentary standards that will be based upon physical metrology.
NIST maintains the technically correct position that torque measurements performed by industry can claim traceability to international standards by combining traceable measurements of the various components from which a torque value is derived - length and force. Secondary torque laboratories in the U.S. either use this approach or procure calibration of their secondary standard torque transducers from a foreign National Measurement Institute (NMI) that offers a torque calibration service.

Industry has been less than satisfied by the NIST position on torque due to widely differing laboratory measurement comparison results; the rates of interlaboratory non-agreement approached 50\% in one study. This discrepancy is due to a lack of uniformity in physical standards, measurement techniques, and documentary standards. The lack of uniformity within the torque community is a technical roadblock to widespread metrological comparability that is not addressed by the current NIST position on torque; hence, the impetus for NIST to become more involved. 


\section{Objectives}

D rovide the tools and measurement methods required to assist U.S. industry and secondary calibration laboratories to identify and resolve the technical causes for the current measurement disagreements.

Improve the secondary calibration system in the U.S. by improving NIST torque measurement capabilities to decrease the measurement uncertainty and provide a reference torque value at a single targeted range, contributing to planning and execution of a torque measurement comparison among U.S. secondary laboratories, analyzing comparison results and identifying areas for improvement in the secondary system, and supporting development of torque metrology standards within the ASTM Mechanical Testing committee.

Conduct bi-lateral or multi-lateral comparisons with key NMI torque laboratories to verify U.S. capabilities according to the international Mutual Recognition Arrangement (MRA). Validate the U.S. torque measurement system and achieve comparability of industrial torque measurements through uniform practices and SI traceability.

\section{Accomplishments}

- Designed and fabricated specialty components and adapters for torque load cell installation to the NIST torque machine.

- Performed the first torque calibration using the complete setup of the NIST torque machine and a commercial torque load cell.

- Evaluated the integrity of the torque machine setup which resulted in a redesign of the adapter parts for improved durability.

- Developed a unique supported torque arm methodology that will decrease torque measurement uncertainties.

\section{Planned Future Accomplishments}

- Complete the design, fabrication, and implementation of the supported torque arm methodology. Perform first initial torque calibration using the supported torque arm methodology and compare results between unsupported vs. supported torque arm calibration and their uncertainties.

- Circulate transducer to selected U.S. secondary laboratories to obtain comparison data, analyze results, identify sources for disagreement, and determine possible methods to achieve more uniform measurements.

\section{Customers and Collaborators}

Boeing Aircraft; Instron Corporation; Interface Inc; HBM Inc; Tovey Engineering 


\section{Automation Update of Force Laboratory Deadweight Machines}

\section{Challenge/Problem Addressed}

alibrations conducted at the laboratory's deadweight force standard workstations are highly dependent on automation systems that were designed and implemented in the late 1980's. Malfunctions have been appearing in the computers, interface components, and voltageratio measuring instrumentation that can no longer be corrected by NIST staff or repair facilities. Simple replacements with current generation instruments cannot be made on an individual unit basis, due to incompatibilities with former generation computers, interfaces, or software. A unified upgrade of these items is necessary to prevent degradation of the force calibration services.

\section{Objectives}

U pdate all force laboratory automation hardware and software to maintain the current capability of force calibration services. A FY2005 modernization effort identified and validated the methods that will now be used to modernize the automation of the remaining five force laboratory deadweight machines.

The primary impact of this work will be to prevent catastrophic and lengthy interruption of NIST force calibration services to customers, who depend upon NIST for traceability in force measurements in all phases of science, industry, and commerce. In addition, improved force calibration efficiencies will result from the automation upgrade, including a greater selection of voltage-ratio measuring instruments and automation of deadweight machine features that currently can only be performed manually. The enhanced automation will also save wear and tear on the hydraulic pumps by allowing shutdown of the hydraulic system after a calibration is complete.

\section{Accomplishments}

- Completed automation update for both the NIST 1.334 $\mathrm{MN}$ and 4.448 MN deadweight machines, including custom control programs, modern data acquisition systems, interfaces to new reference multimeters, and improved user interfaces.

- Evaluated and integrated a laser measurement sensor into the $113 \mathrm{kN}$ dead weight machine to replace a failed optical sensor (infrared shadow sensor). The sensor will allow us to operate the machine in split-frame mode under full automation whenever this machine upgrade is completed in the future.

- Outfitted the $27 \mathrm{kN}$ machine that was automated earlier as the pilot project with programs to allow calibration with the new voltmeters.

\section{Planned Future Accomplishments}

- Complete automation update for the NIST $498 \mathrm{kN}$ and $2.2 \mathrm{kN}$ deadweight machines.

- Complete automation update for the NIST 113 kN deadweight machine.

\section{Customers and Collaborators}

Boeing Aircraft; Instron Corporation; Interface Inc; HBM Inc; Tovey Engineering; Morehouse; Lockheed; United Technologies 


\section{Implement Robotic Mass Measurements in SP250 Services}

\section{Challenge/Problem}

\begin{abstract}
raditional implementation of SP250 mass calibration services require the physical presence of NIST staff during the measurement of a customer's mass standards using weighing designs. As such, the human operator's presence introduces thermal effects and operator-dependent repeatability issues into the measurement, as well as resulting in a larger turnaround time for the service and a larger statistical uncertainty. The major statistical barrier with the existing robotic balances is that some of the currently used weighing designs published in NIST Technical Note 952 are not feasible for the robotic balances. New (orthogonal or non-orthogonal) weighing designs must be constructed, tested, and evaluated.
\end{abstract}

\section{Objectives}

D evelop and implement the procedures required to have fully automated / robotic mass measurement services in the range from $1 \mathrm{mg}$ to $64 \mathrm{~kg}$ and for solid density measurements of mass standards in the range of $1 \mathrm{~kg}$ to $10 \mathrm{~g}$. This will eliminate human-induced errors, shorten turnaround time (since measurements can be set up to be performed outside of regular business hours), and possibly lower the cost for the service in the future. Develop, test, and evaluate weighing designs, uncertainty budgets, and statistical process control proce- dures. Develop quality system for newly developed methods and incorporate into the SP250 calibration services for customers.

\section{Accomplishments}

- Implemented the $64 \mathrm{~kg}$ robotic balance for SP250 services for weights in the $10 \mathrm{~kg}$ to $64 \mathrm{~kg}$ range.

- Developed new weighing designs for the $1 \mathrm{~kg}$ to $1 \mathrm{mg}$ range; they are currently under testing and evaluation.

\section{Planned Future Accomplishments}

- Complete testing, analysis, and evaluation of new weighing designs.

- Develop the statistical process control procedures uncertainty budget in compliance with the ISO guide to measuring uncertainty.

- Develop all documentation required for the ISO 17025-compliant quality manual for mass calibration procedures.

- Deliver robotic measurement services for SP250 tests in the range of $1 \mathrm{mg}$ to $1 \mathrm{~kg}$ and fully automated density measurements.

\section{Customers and Collaborators}

U.S. State Weights \& Measures Laboratories; Army, Navy, and Air Force Calibration Laboratories; Boeing; Lockheed; Amgen; Troemner; Mettler-Toledo; Los Alamos National Laboratory

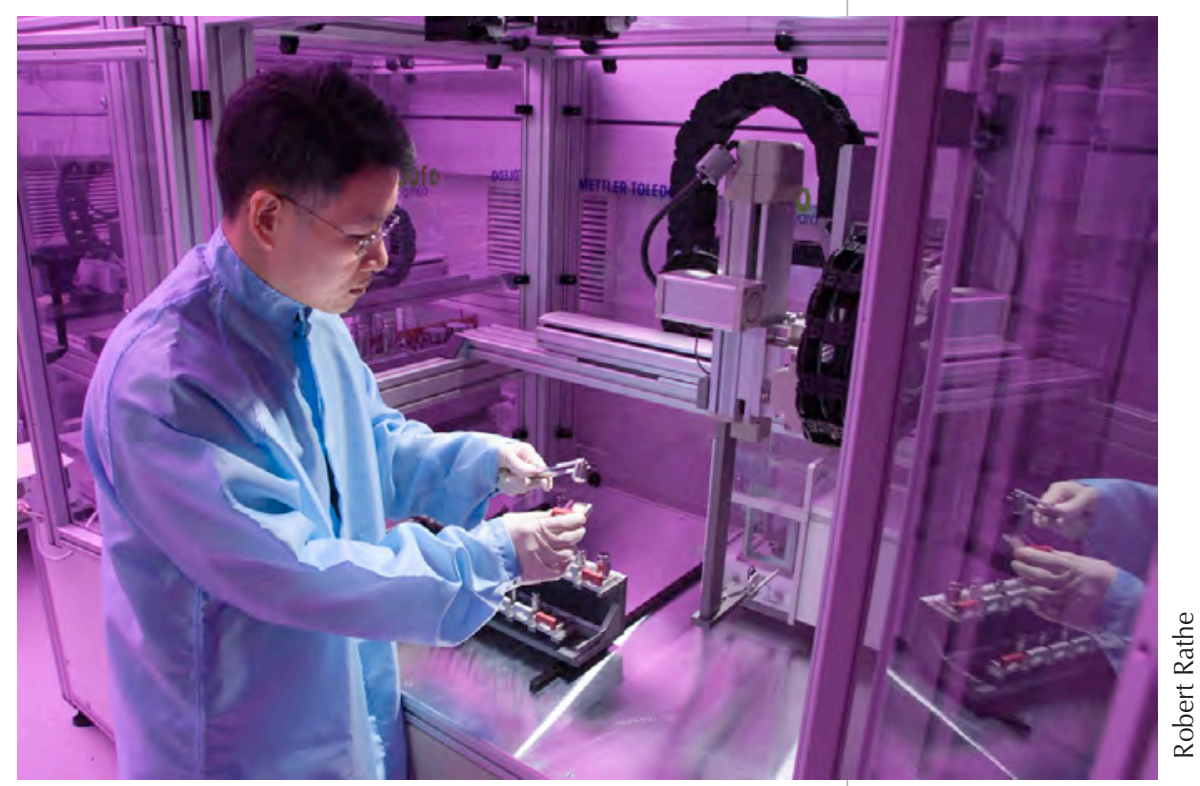




\section{Next-Generation Acoustic Measurements of Microphones}

\section{Challenge/Problem Addressed}

T he challenge is to meet the new and more demanding needs of NIST calibration customers, such as the U.S. Army and U.S. Air Force primary standards laboratories, Lockheed-Martin Corporation, Mine Safety and Health Administration, and measuring instrument manufacturers, such as Bruel \& Kjaer North America, who are increasingly sending new types of laboratory microphones to NIST for calibration. These microphones can meet new and more demanding customer needs because the microphone properties enable them to be calibrated to smaller uncertainties over a wider frequency range than those of current NIST SP250 measurement services. Microphones calibrated at NIST via these services are used by customers to provide traceability for many thousands of other acoustical measuring instruments used in critical measurements for product design and conformance, worker and military personnel safety, and health care. Provision of next-generation microphone pressure calibration services with smaller uncertainties by NIST will enable customers to meet competitive and contractual pressures to provide state-of-the-art measurements, to satisfy legal metrology needs and avoid costly litigation and damages, and to achieve reliable measurements of noise emission close to regulated or mandated limits. As examples: (1) a $1 \mathrm{~dB}$ difference in noise measurements for an aircraft certification could be worth $\$ 100$ million; and (2) air conditioning units that do not meet new classroom noise standards for school construction could be shut out of that very large market.

\section{Objectives}

D evelop and provide next-generation acoustical measurement services that meet the needs of our customers by reducing our measurement service uncertainties for pressure-field calibrations of next-generation laboratory standard microphones (ANSI/IEC Types LS2aP and LS1Pn).

\section{Accomplishments}

- Completed the design, test, and integration of the measurement system, software, and procedures required for reciprocity calibrations of Type LS2aP laboratory standard microphones from $31.5 \mathrm{~Hz}$ to 20 $\mathrm{kHz}$ in plane-wave air-filled couplers. This measurement capability halves our measurement uncertainties in the most critical frequency range.

- Calibrated a Type LS2aP microphone for the Air Force Primary Standards Laboratory from $31.5 \mathrm{~Hz}$ to $20 \mathrm{kHz}$ using the newly developed next-generation measurement service.

\section{Planned Future Accomplishments}

- Complete the design, test, and integration of the measurement system, software, and procedures required for reciprocity calibrations of Type LS1 Pn laboratory standard microphones from $50 \mathrm{~Hz}$ to $10 \mathrm{kHz}$ in planewave air-filled couplers.

- Provide a next-generation measurement service for Type LS1Pn microphones that halves our measurement uncertainties in the most critical frequency range.

\section{Customers and Collaborators}

U.S. Army and Air Force Primary Standards Labs; Bruel \& Kjaer North America; Mine Safety and Health Administration; Lockheed-Martin Corporation; Wyle Laboratories; Honeywell 


\section{Low Frequency Vibration Metrology}

\section{Challenge/Problem Addressed}

\begin{abstract}
1 ccurate measurement of low frequency vibration is critical for applications such as inertial guidance systems, assessment of whole-body vibration in vehicles, and monitoring of seismic vibrations in nuclear reactors and other structures. Specific industries that require accurate low frequency vibration measurements include the transportation industry, off-road heavy equipment manufacturers, nuclear power industry, building and construction industry, and transducer manufacturers. The current NIST low frequency vibration calibration system is outdated, requires significant time and expense for maintenance, and is inefficient in its operation.
\end{abstract}

\section{Objectives}

stablish a new low frequency measurement capability and calibration service that covers lower frequencies $(0.2 \mathrm{~Hz}-200 \mathrm{~Hz})$ with reduced uncertainty for the entire target frequency range and retire the existing exceedingly high-maintenance and operationally-inefficient low frequency vibration system that is currently used for much of the target range.

Validate the new measurement capability and procedures and establish the basis for using the new system for customer calibrations by conducting a measurement comparison with other National Measurement Institutes.

\section{Accomplishments}

- Designed the low frequency calibration system and acquired all system components (e.g., temperature sensors, zero-position controllers, data acquisition hardware and software).

- Designed, fabricated, installed, and tested the optical components for measuring the motion of the two shakers of the new calibration system.

- Performed extensive measurements to characterize the motion of the new vibration generators as a function of frequency and amplitude, including analysis of rocking motion (yaw, pitch, and roll), transverse motion, harmonic distortion, and signal-to-noise ratios.

- Analyzed various components of the measurement uncertainty budget, including the degree of vibration isolation between system components, the calibration of the function generator, and the magnetic flux densities produced by the vibration generators.

\section{Planned Future Accomplishments}

- Complete operational testing and analysis of the new low-frequency vibration metrology system and establish final measurement uncertainty budget.

- Conduct the international measurement comparison in low-frequency vibration.

- Develop all quality system documentation to transition the new measurement capability to a measurement service for NIST customers.

\section{Customers and Collaborators}

NASA; Air Force and Navy Primary Standards Labs; Sandia National Laboratory; Duke Energy; Rockwell Automation; Raytheon; Boeing; Caterpillar; John Deere; Endevco; Kistler Instruments 
Standards Leadership And Participation

\begin{tabular}{|c|c|c|c|}
\hline & Mass & Force & Acceleration \\
\hline $\begin{array}{l}\text { National } \\
\text { Standards }\end{array}$ & $\begin{array}{l}\text { - ASTM E41.06 Weighing } \\
\text { Devices }\end{array}$ & $\begin{array}{l}\text { - ASTM E28 Mechani- } \\
\text { cal Testing }\end{array}$ & $\begin{array}{l}\text { - ANSI S2 Mechanical Vibration \& Shock (Vice- } \\
\text { Chair) } \\
\text { WG2 Terminology (Chair) } \\
\text { WG5 Calibration \& Use of Instruments (Chair) } \\
\text { - ANSI/ASA US TAG for ISO TC108 (Chair) } \\
\text { - ANSI/ASA US TAG for ISO TC108/SC3 Use and } \\
\text { Calibration of Mechanical Vibration \& Shock Mea- } \\
\text { suring Instruments (Chair) }\end{array}$ \\
\hline SIM & $\begin{array}{l}\text { - Metrology Working } \\
\text { Group } 7 \text { Mass \& Related } \\
\text { Quantities }\end{array}$ & $\begin{array}{l}\text { - Metrology Work- } \\
\text { ing Group } 7 \text { Mass \& } \\
\text { Related Quantities }\end{array}$ & - Metrology Working Group 9 Acoustics \& Vibration \\
\hline OIML & $\begin{array}{l}\text { - OIML TC9 Instruments } \\
\text { for Measuring Mass \& } \\
\text { Density } \\
\text { - OIML TC9 SC3 Weights } \\
\text { (Tech Advisor) }\end{array}$ & $\begin{array}{l}\text { - USNWG / OIML TC9 } \\
\text { /WG1 Load Cells }\end{array}$ & \\
\hline $\begin{array}{l}\text { CIPM Consul- } \\
\text { tative Com- } \\
\text { mittees }(\mathrm{CC})\end{array}$ & $\begin{array}{l}\text { - CC for Mass and Related } \\
\text { Quantities (CCM) WG on } \\
\text { Mass \& Density }\end{array}$ & $\begin{array}{l}\text { - CC for Mass and } \\
\text { Related Quantities } \\
\text { (CCM) WG on Force }\end{array}$ & $\begin{array}{l}\text { - CC for Acoustics, Ultrasound, and Vibration } \\
\text { (CCAUV) }\end{array}$ \\
\hline ISO/IEC & Not generally applicable & $\begin{array}{l}\text { - ISO TC } 201 \text { SC9 } \\
\text { Scanning Probe } \\
\text { Microscopy }\end{array}$ & $\begin{array}{l}\text { - ISO TC } 108 \text { Mechanical Vibration \& Shock (Chair } \\
\text { of US Delegation) } \\
\text { - WG } 1 \text { Terminology } \\
\text { - WG } 26 \text { Signal processing methods for analysis of } \\
\text { mech. vibration \& shock (Convener) } \\
\text { - SC3 Use and Calibration of Vibration \& Shock } \\
\text { Instrumentation (Chair of US Delegation) } \\
\text { - SC3/WG6 Calibration of Vibration \& Shock Trans- } \\
\text { ducers } \\
\text { - SC3/WG10 Vib. Condition Monitoring Transduc- } \\
\text { ers and Instrumentation }\end{array}$ \\
\hline
\end{tabular}


- SAE Emergency Vehicle Siren (J1849)

\section{Acoustics}

- ANSI/ASA S1 Acoustics

- WG 1 Standard Microphones \& Their Calibration (Chair)

- WG21 EMS of Acoustical Instruments

- ANSI/ASA S3 Bioacoustics

- WG48 Hearing Aids

- ASA Committee on Standards (ASACOS)

Task Force (Vice-Chair)

- SAE Emergency Warning Lights and Devices

- ANSI US TAG for IEC TC87

Ultrasonics
Optics

- ANSI/Optics and Electro-Optics Standards Council (OEOSC) US TAG for ISO TC 172 Optics and Photonics

\section{Not generally applicable} tics \& Vibration

\section{Manufacturing Technology}

- ANSI/ASME B5 Machine Tools

- ANSI/ASME B5/TC52 Machining \& Turning Centers

- ANSI/ASME B5/TC56 Information Technology for Machine Tools (Chair)

- ANSI/ASME B89.3.4 Axes of Rotation

- IEEE P1451 Smart Transducer Interface for Sensors \& Actuators; P1451.0, .1, .2, .3, .4, .5, \& .7 WGs

- IEEE 1588 Precision Synchronization for Networked Measurement \& Control WG

- IEEE I\&M Society TC9 Sensor Technology Committee (Chair)

- IEEE-SA Standards Committee (I\&M Society liaison)

- ASTM F42 Additive Manufacturing Technologies

Not generally applicable

Not generally applicable

- US National WG for OIML TC13

Not generally applicable

Measuring Instruments for Acoustics

and Vibration (Technical Advisor)

\section{- CC for Acoustics, Ultrasound, and} Vibration (CCAUV)
Not generally applicable
- IEC TC 29 Electroacoustics (Chair of US Delegation)

- IEC TC 29/WG5 Measurement Microphones

- US National Committee for IEC (Member, as Technical Advisor for TC 29 Electroacoustics)

\section{- ISO TC 172 Optics and Photonics}

- ISO TC 39 Machine Tools

- ISO TC 39/SC2 Test Conditions for Machine Tools (Secretariat)

- ISO TC 39/SC2/WG1 Geometric Accuracy of Machine Tools

- ISO TC 39/SC2/WG3 Test Conditions for Machining Centers

- ISO TC39/CS2/WG4 Test Conditions for Turning Centers

- ISO TC 39/SC2/WG6 Thermal Effects (Convener)

- ISO TC39/SC2/WG7 Capability of Machine Tools

- ISO TC 184/SC4 Industrial Data 


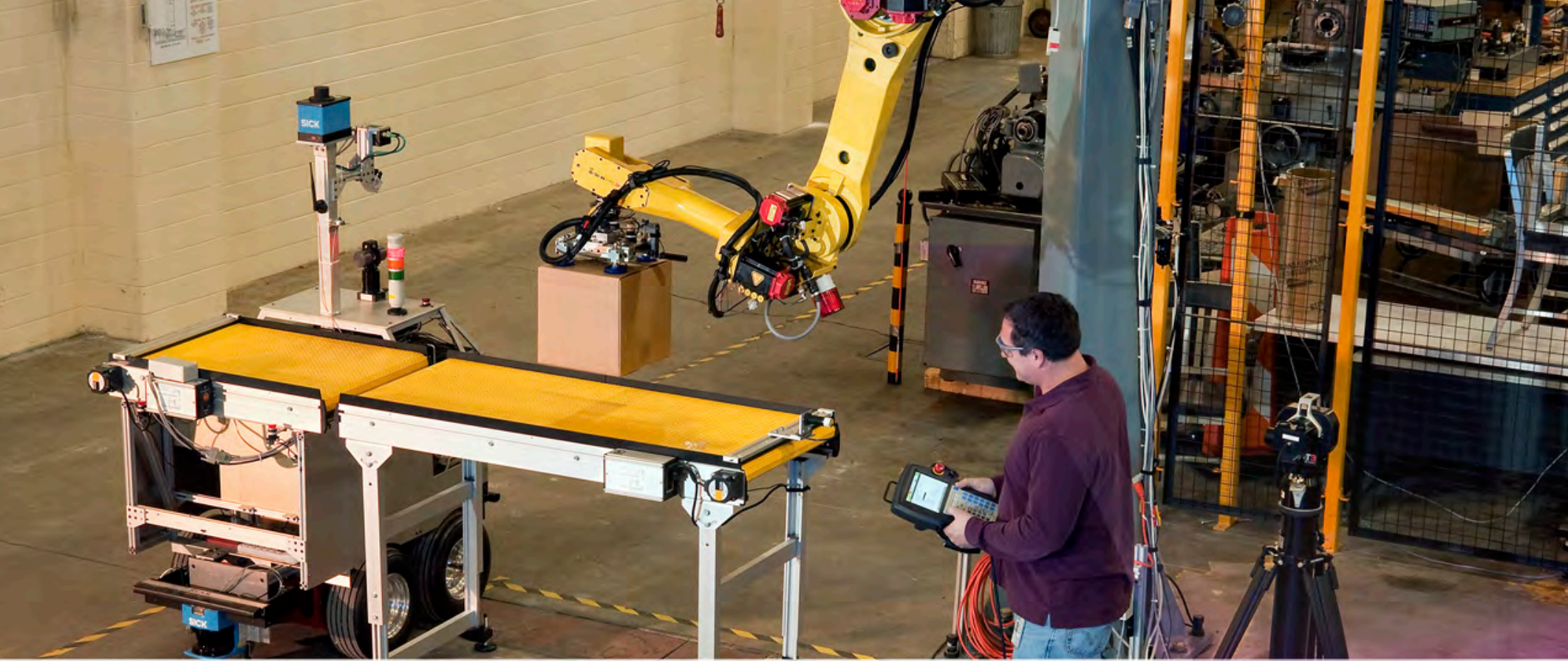

\section{Developing the measurements and standards infrastructure needed for the application of intelligent systems}

\section{Intelligent Systems}

\section{Overview}

T he Intelligent Systems Division supports a future in which U. S. manufacturing becomes more accurate, agile, intelligent, interoperable, and reconfigurable through constant innovation. Our work promotes the development of measurement science and interoperability standards that will enhance manufacturing robotics and automation equipment and the underlying industrial control systems.

Federal and industry groups have specifically stressed the importance of innovation in robotics, manufacturing automation, and control systems for maintaining U. S. primacy in innovation and high-value-added manufacturing. For example, key technologies for the future of manufacturing cited by a National Research Council publication ${ }^{1}$ include interoperability/interoperability standards; pervasive and adaptive process control; security and other protection systems; modeling and simulation technology; and the incorporation of intelligence into processes and products. The President's Framework for Revitalizing American Manufacturing recognizes the importance of "developing advanced robotics technologies that allow the U.S. to retain manufacturing, and respond rapidly to new products and changes in consumer demand." ${ }^{2}$
Chief:

Elena Messina (Acting)

\section{NIST}

100 Bureau Drive

Stop 8230

Gaithersburg, MD

20899-8230

Phone: 301 975-3510

Fax: 3019909688

Email:

elena.messina@nist.gov

1 National Research Council, "New Directions in Manufacturing: Report of a Workshop," http://www.nap.edu/catalog/11024.htm, 2004.

2 Executive Office of the President, "A Framework for Revitalizing American Manufacturing," December 2009. 
The 2011 Budget proposed by the Administration states that "standards and measurements can improve the societal benefits from emerging technologies by allowing for interoperability, promoting efficiencies, and enabling better manufacturing methods." ${ }^{\prime 3}$

Robotics holds tremendous untapped potential in manufacturing. It is estimated that $90 \%$ of potential users have not yet adopted robotics. ${ }^{4}$ Development and adoption of next-generation robotics technology - supported and accelerated by new measurements and performancebased standards - will "enable the nation to maintain a strong, globally competitive manufacturing base, ensure our continued economic growth, and help safeguard our national security." "Robotics is a key transformative technology that can revolutionize manufacturing... the promise of flexible automation and automation for mass customization has not been realized except for special cases.... Robots [need] to be smarter, more flexible, and able to operate safely in less structured environments shared with human workers ${ }^{6} . "$

Current and emerging user communities lack any means whatsoever of specifying quantitatively what performance they need from a robot. Nor are there any means of objectively and reproducibly measuring how well robots or their components meet a given set of requirements. As robots and other manufacturing equipment become more flexible and adaptable, and work in closer unison with humans, safe operations become a paramount issue. It is essential for progress in the area of human-robot collaboration that there be consensus standards for measuring the performance of safety sensors, control systems, and other equipment for industrial robot arms and for mobile robots.

Other manufacturing equipment that is much more pervasive on the shop floor needs to also support greater agility. Agility requires well-defined interoperability standards that permit reconfiguration of equipment without necessitating costly and unreliable translators.

3 Office of Science and Technology Policy, R\&D Summary Investing in the Building Blocks of American Innovation, http:/ www.whitehouse.gov/sites/default/files/fy2011 rd\%20final.pdf February 2010.

4 World Robotics 2006 statistics summarized by H. Christensen in presentation "New' Applications in Industrial Robotics," RoboBusiness 2007, Boston, MA, May 2007.

5 "A Roadmap for US Robotics: From Internet to Robotics," Computing Community Consortium (CCC) and Computing Research Association (CRA) report, May 21, 2009, http://www. us-robotics.us/reports/CCC Report.pdf

6 ibid
The interoperability standards must allow for rich information exchange to enable new programs to be executed on machines without the typical trial and error fine-tuning currently needed. With greater information flowing through the entire work cell and beyond, adjustments and corrections can be made to allow parts to continue to be produced correctly without incurring costly human intervention or scrap.

As factory equipment becomes more tightly networked within the work cells as well as with external systems, security and performance issues grow in importance. The vision of more agile and efficient enterprises relies on manufacturing equipment and software systems that have control systems that communicate vast quantities of information through shop floor networks. These industrial control systems and their networks must have well-characterized and reliable performance and must be impervious to security threats. Beyond traditional manufacturing enterprises, the Nation's critical infrastructure also relies on similarly complex and vulnerable industrial control systems. Concerted efforts in characterizing and securing industrial control systems can therefore benefit numerous sectors. In particular, there is an urgent mandate for making the Smart Grid secure and interoperable. NIST's expertise in working with the critical infrastructure networks and control systems is being leveraged for the new Smart Grid initiative.

Referring to the Drivers and Needs for U. S. Manufacturing Innovation and Competitiveness identified by the Manufacturing Engineering Laboratory, the Intelligent Systems Division seeks to address the increasing pace of technological change, which encompasses product and process innovation, as well as reduction in time to market, higher quality and better performance of customized products, increased productivity and reduced costs, and new safety and security challenges.

The Intelligent Systems Division tackles the challenges posed in attaining this vision of the future by partnering with industry and academia to ensure that innovation in manufacturing systems is well-characterized and quantifiable (through development of a measurement science), open, extensible, and reconfigurable (through development of interoperability standards), as well as safe and secure (through industrial control network security standards). To target these challenges, our Division's work is organized into three programs. The Measurement Science for Manufacturing Robotics and Automation Program provides means of characterizing, 
measuring, and assuring the performance of advances in technologies that make possible manufacture of higher quality and more complex parts and assemblies through predictable, well-characterized manufacturing equipment. Flexible, reconfigurable manufacturing systems and model-based manufacturing require well-defined and rich information transfer, which is addressed by the Intelligent Manufacturing Robotics and Automation Interoperability Standards Program. The Intelligent Manufacturing Industrial Control Systems and Network Standards Program responds to the need for safe, secure, and reliable manufacturing control systems. The Intelligent Systems Division is world-renowned for its accomplishments in development of measurement science, tools, and interoperability solutions for leading-edge mobile robots and other complex automation that has been applied to defense, transportation, and security. We bring this core competence to bear in our work within the manufacturing domain.

\section{Mission}

To develop the measurements and standards infrastruture needed for the application of intelligent systems

\section{Facilities and Equipment}

SD is expanding the set of equipment and facilities that support its measurements and infrastructure mission. ISD uses unique facilities and tools to define performance metrics and measurement methods and to serve as testbeds for industry and researchers.

Dimensional Metrology Interoperability Testbed: Supports development and validation of new data and interface protocols and test suites for quality measurement information.

\section{Smart Machining And Machine Tool Interoperability}

Testbed: Supports validation and testing of emerging data standards for machining equipment.

Industrial Control Networking and Cybersecurity Testbed: Supports development and testing of new network performance standards
Industrial Robotics Measurement Science Testbed:

Supports development and validation of new performance test methods for advanced industrial robot arms and mobile robots.

Micro- through Nano-devices 3D Metrology Testbed: Provides 1D, 2D, and 3D assembly and positioning metrology and test facilities using high-precision MEMS stages, controllers, nanoprobes and calibration devices.

Nanorobotics and NEMS Testbed: Develops foundational sensing and measurements for nanorobotics and

NEMS with an emphasis on nanoscale displacement and force.

Indoor and Outdoor Position Measurement and Tracking

Facilities: Permits evaluation of sensors and algorithms for mobile robots through a variety of dynamic vehicle and sensor data collection efforts. This set of facilities includes standard and draft test method installations, as well as several unique buildings and outdoor areas appropriate for applicationspecific scenarios for evaluating robot performance. Examples include military and bomb squad operations.

State-of-the art 3D and 6D sensors: Support characterization and development of test methods and reference data sets (ground truth) against which to measure sensing and positioning accuracy, both statically and dynamically.

\section{Facilities and Equipment Challenges}

IST is currently slated to relinquish the site that houses the Indoor and Outdoor Position Measurement and Tracking Facilities in the coming years. A new building will be constructed on campus using American Recovery and Reinvestment Act funding. However, the allocated funding is insufficient to replicate current square footage available offsite. 
ISD Program

\section{Intelligent Manufacturing Industrial Control Systems \& Network Standards}

The introduction of information technologies, such as wireless and Ethernet, into critical industrial infrastructure systems has provided greater and more agile control over electric power, water, oil, natural gas and manufacturing facilities around the country. The modernization of these systems, however, requires that security measures, termed "critical infrastructure protection" (CIP), be implemented to protect them from accidental and intentional incidents. This program develops the security standards and guidelines for the computerized systems that control the nation's utilities as well as other staples of daily life. It also drives development of new security tools for future systems.

\section{Staff}

3 NIST Staff

\section{Total FTEs}

\section{Challenge}

\begin{abstract}
afe, secure, and reliable industrial control systems
(ICS) are essential for U.S manufacturers and other critical infrastructure. As inter-connectivity grows, the challenges in guaranteeing the security of the most critical systems increase correspondingly. The same problems arise as with conventional business information technology, but are made more difficult by the increased demands for reliability and real-time execution required by industrial automation. It is not enough to apply IT security solutions to the computers in the control room great care must be taken to ensure that equipment spanning decades of 24/7 operation remain operating.
\end{abstract}

\section{Technical Strategies}

T

he program has three primary strategies to ad-

dress the challenges: secure existing systems; drive toward built-in security for new systems that will be deployed in the future; and develop security and performance test methods for these systems.

\section{Results \& Impacts}

- Participated in the development of NIST Special Publication (SP) 800-53, Recommended Security Controls for Federal Information Systems and Organizations, Revision 3 to standardize the security controls for federally owned/operated ICS. NIST SP 800-53, Revision 3 has been accessed over 1,250,000 times since its release in February 2009.

- Participated in the development of the North American Electric Reliability Corporation (NERC) CIP, Revision 2 cyber security standards, CIP-002-2 - CIP009-2, which were approved by the NERC Board of Trustees on May 6, 2009. These revised NERC CIP cyber security standards will play a major role in securing the North American Bulk Electric System and the future Smart Grid.

- Participated in the development of the NIST Framework and Roadmap for Smart Grid Interoperability Standards, Release 1.0 report that identifies a foundational set of standards for grid interoperability and security, as well as high-priority action plans to fill gaps where additional standards are needed.

- Developed NIST Special Publication (SP) 800-82, Guide to Industrial Control Systems (ICS) Security that has been accessed over 750,000 times since the initial public draft. NIST SP 800-82 is recognized by the 
community as one of the most valuable ICS security documents available today and is heavily used and referenced in the private sector, which owns/operates $90 \%$ of the critical infrastructure.

- Developed and published version 1.0.1 of the Industrial Ethernet Network Performance (IENetP) Test Tool in April 2009. This test tool helps product developers improve the reliability of their product(s) before they are shipping to customers.

\section{Publications}

ANSI/ISA-99.02.01-2009 - Security for Industrial Automation and Control Systems: Establishing an Industrial Automation and Control Systems Security Program

Industrial Control System (ICS) Security Standards and Guidelines, World Cyber Security Summit,

Kuala Lumpur, Malaysia, May 20-21, 2008

Testing, Comparing Industrial Ethernets, InTech

Magazine, August 2009, Cover Story, pp. 12-15.

\section{Contact Information}

Keith Stouffer

301-975-3877

keith.stouffer@nist.gov

\section{Awards and Leadership}

\section{Board Memberships}

Staff

Jim Gilsinn

\section{Board Membership}

Managing Director of 5 Committees, International Society of Automation's (ISA's) Standards \& Practices (S\&P) Board

\section{Leadership}

\section{Staff}

Jim Gilsinn

$\operatorname{Jim}$ Gilsinn
$\operatorname{Jim}$ Gilsinn

Keith Stouffer

Keith Stouffer

Keith Stouffer

Keith Stouffer

Keith Stouffer

Keith Stouffer

\section{Leadership}

Editor, ISA-99 Committee on Security for Industrial Automation \& Control Systems, Working Group 2 and 7 Leadership Committee, ISA-99

Chair, ISA Expo Security Conference Committee 20072009

Coordinator, ODVA, Inc. EtherNet/IP Implementors Workshop

Technical Advisor, US TAG for the IEC TC65 committee Leadership committee, ISA-99

Co-Chair, NIST Smart Grid Industry-to-Grid (I2G)

Domain Expert Working Group

Coordinator, NERC Critical Infrastructure Protection (CIP) Standards Drafting Team Kick Off Meeting, NIST,

October 2008

Technical Advisor, CIGRE B5 committee

Invited speaker at over 20 international conferences and forums 
Intelligent Manufacturing

Industrial Control Systems \&

Network Standards Projects

\section{Federal Industrial Control System Security}

\section{Challenge/Problem Addressed}

afe, secure, and reliable federally owned or oper-

ated industrial control systems are essential for the National critical infrastructure. Currently, federal and private sector organizations in the electric sector follow different security standards.

\section{Objectives}

- Provide federal organizations that operate industrial control systems (e.g., Bonneville Power Administration, Bureau of Reclamation, Federal Aviation Administration, Tennessee Valley Authority), the required technical guidance and baseline security safeguards to secure these systems, significantly reducing the risk of compromise and negative impacts to federal critical infrastructure.

- Actively work with the North American Electric Reliability Corporation (NERC) and Federal Energy Regulatory Commission (FERC) to facilitate the development of security standards for the North American electric sector that are commensurate with the security standards for federal agencies that operate industrial control systems in the electric sector.

\section{Accomplishments}

- NIST SP 800-53, Revision 3 was developed to standardize the security controls for federally owned/ operated ICS. This standard, "Recommended Security Controls for Federal Information Systems," is the first, and currently only, security standard that addresses both general information technology (IT) systems and ICS. This allows the federal agencies, as well as the private sector if desired, to use one document to determine the proper security controls for their IT systems as well as to effectively secure their industrial control systems while addressing their unique requirements. NIST SP 800-53, Revision 3 is also actively being vetted within the ISA-99 WG4 (Industrial Automation and Control Systems Security) and IEC TC65/WG10 (Security for Industrial Process Measurement and
Control) standards committees, which are developing private sector international cyber security standards for industrial control systems. NIST SP 800-53, Revision 3 has been accessed over 1,250,000 times since its release in February 2009.

- Developed NIST Special Publication (SP) 800-82, Guide to Industrial Control Systems (ICS) Security which has been accessed over 750,000 times since the initial public draft. NIST SP 800-82 is recognized by the community as one of the most valuable ICS security documents available today and is heavily used and referenced in the private sector, which owns/ operates $90 \%$ of the critical infrastructure.

- Participated in the development of NERC CIP, Revision 2 cyber security standards, CIP-002-2 - CIP-009-2 (Cyber Security), which were approved by the NERC Board of Trustees on May 6, 2009 after passage by the electric industry with a quorum of $94.37 \%$ and an $88.32 \%$ approval rating. These revised NERC CIP cyber security standards will play a major role in securing the North American Bulk Electric System and the future Smart Grid. These standards cover Asset Identification, Management Controls, Personnel and Training, Electronic Perimeters, Physical Security, Incident Reporting and Recovery.

\section{Planned Future Accomplishments}

- Contribute to the development of NERC CIP, Revision 4 cyber security standards which will address a larger portion of the electrical grid that will play an even greater role in securing the North American Bulk Electric System and the future Smart Grid.

\section{Customers and Collaborators}

Bonneville Power Administration, Bureau of

Reclamation, CIGRE - International Council for Large Electric Systems, Department of Homeland Security, Federal Aviation Administration, Federal Energy Regulatory Commission, Instrumentation, Systems, and Automation Society (ISA), North American Electric Reliability Corporation, Tennessee Valley Authority 
Private Sector Industrial Control System Security

\section{Challenge/Problem Addressed}

Dublic and private sector critical infrastructure organizations currently have different security requirements and safeguards in place, which could lead to vulnerabilities and inefficiencies. A harmonized set of security requirements and baseline security safeguards will provide consistent levels of protection for both public and private sector critical infrastructure.

\section{Objectives}

- Provide private sector organizations that operate industrial control systems the technical guidance and baseline security safeguards to secure these systems, significantly reducing the risk of compromise and negative impacts to these systems.

- Provide leadership within standards development organizations (e.g. ISA-99 Industrial Automation and Control System Security Committee) to drive the adoption and implementation of common security requirements and baseline security safeguards.

\section{Accomplishments}

- Developed ANSI/ISA-99.02.01-2009 Security for Industrial Automation and Control Systems: Establishing and Industrial Automation and Control Systems Security Program, which provides comprehensive guidance on developing an industrial control system security program. Contributed to the development and publication as the lead editor of the first normative standard from the International Society of Automation (ISA) on Security for Industrial Automation and Control Systems, ANSI/ISA-99.02.01-2009 establishing an Industrial Automation and Control Systems Security Program. This is the first normative standard in the ISA-99 series of standards. The standard is also being published through the International Electrotechnical Commission (IEC) in the 62443 series of standards. This standard applies to the much broader commercial ICS base and leads the way for comprehensive and universal cybersecurity.

\section{Planned Future Accomplishments}

- Update ANSI/ISA-99.02.01-2009 to address comments from the International Electrotechnical Commission (IEC) 62443 committee.

- Revise ISA-99.02.01 to better align with the IEC 27000 series of standards covering Information Technology - Security Techniques. Alignment with the IEC paves the way for commercial availability of security products that directly support the needs of the industrial base.

\section{Customers \& Collaborators}

ChevronTexaco, Department of Homeland Security, Dow, ExxonMobil, General Motors, Georgia-Pacific, International Electrotechnical Commission, ISA, Procter \& Gamble 


\section{Industrial Performance Test Methods and Tools}

\section{Challenge/Problem Addressed}

lack of performance test methods has prevented the wider acceptance of standards by industrial control systems users and developers. This limits advancements in performance and reliability of manufacturing and other enterprises that rely heavily or entirely on networks for conducting their operations.

Therefore, industrial performance test methods increase standards adoption by providing users with a way to determine standards compliance and to verify that the standards are technically sound and feasible. These performance test methods will ensure device reliability and that users can more easily estimate overall network performance based on statistically sound device performance metrics.

\section{Objectives}

- Work with device vendors to define performance measures and validate the test methods to promote confidence in and adoption of commonly-accepted performance test methods.

- Develop tools that help capture and visualize performance data to increase awareness and understanding by the community, leading to improvements in device and network performance.

\section{Accomplishments}

- Developed and published version 1.0.1 of the Industrial Ethernet Network Performance (IENetP) Test Tool in April 2009. This test tool is capable of analyzing network capture files and reporting a standard set of performance metrics. Vendors can use this information to improve the reliability of their products, such as controllers, motors, and sensors. Users can utilize the standardized metrics to compare the performance characteristics of multiple vendor's products in common terminology, similar to the automobile fuel economy tests.

- IENetP software being used by industrial vendors and users. The software has been downloaded from SourceForge over 375 times. Multiple vendors have stated that they are using the software in-house to analyze the performance of their devices during their development phase. The vendors have all had comments and feature requests about how NIST can improve the software for their particular need.

- Eight presentations have been made to the EtherNet/IP Implementors Workshop. The main vendor supporting EtherNet/IP has started recommending the IENetP to their OEM customers to improve their endproducts and to their users to help improve their site installations.

- Gilsinn, J., Johnson, F., Test Tool for Industrial Ethernet Network Performance, presented at 55th International Instrumentation Symposium (IIS), June 1-5, 2009. This paper was well received at the IIS conference and was turned into a cover story article for InTech magazine. Gilsinn, J., Johnson, F., Testing, Comparing Industrial Ethernets, InTech Magazine, August 2009, Cover Story, pp. 12-15. 


\section{Planned Future Accomplishments}

- Expand the mathematical analysis capability of the performance testing system to better characterize the performance of the device and/or system being tested. This will also include a re-analysis of the performance metrics to determine how the more detailed mathematical analysis can be presented to the user in a cohesive and understandable way.

- Develop additional test methodologies based on industry requirements to increase the overall performance analysis capability of the test system. These new test methodologies will allow for additional protocols and physical media to be investigated, including a greater number of industrial protocols, wireless protocols, and web-based protocols.

- Develop the protocol analysis capability to analyze a greater number of industrial protocols. The industrial Ethernet market currently consists of 10-15 full protocols with a multitude of supporting protocols, including but not limited to: Modbus/TCP, Profinet, Foundation Fieldbus HSE, ISA-100.11a, IEEE-802.11/ WiFi, Zigbee, and OPC. By expanding the number of protocols, the IENetP software will benefit a larger number of national and international vendors and users.

- Provide users with both test tools and test plans for conducting performance testing at their facility with their equipment. The ability for vendors to conduct tests themselves greatly speeds up the testing process, and makes learning from failure less risky. These test tools will be designed to be as open-source and freely available as possible.

\section{Customers}

Chrysler, Ford, General Motors, HMS Industrial Networks, Industrial Security Compliance Institute (ISCI), ODVA, Inc., Rockwell Automation, Schneider Electric Siemens, United Stated Council for Automotive Research (USCAR), Woodhead 


\section{Intelligent Manufacturing Robotics and Automation Interoperability Standards}

In a global economy with competition from developing countries that have much lower labor rates, innovation is America's best hope to remain competitive. Innovation opens up new business opportunities and creates new ways to put people to work. Innovation means answering "what if" and it ought to be easy to answer questions like "what if we could move our custom applications between robots from all our vendors? What if we can apply all our post-process measurement software to inprocess measurements? What if we could use the same data for cutting non-uniform materials that we use for modeling them?" By making it easy to answer these questions, we can reduce the cost of innovation.

\section{Staff:}

4 NIST staff

3 guest researchers

5.5 total FTEs

\section{Challenge}

f manufacturers are bound by single-vendor stovepiped products, they will end up with single-use stovepiped innovation. Breaking free means being able to put together best-in-class products from niche technology vendors to build creative solutions, making it easier for U.S. manufacturers to innovate on the shop floor and deliver products found nowhere else at affordable costs. Enabling interoperability - putting it all together quickly and easily - is the goal of this program. The problem is hard because the factory floor is where engineers from many different disciplines come together in a tangle of incompatible equipment, and are tasked with making things work on tight schedules. The different disciplines have different perspectives on the information that is necessary; hence it is a tremendous challenge to determine which data are needed, and how to express them unambiguously and efficiently.

\section{Technical Strategy}

tandards are a prerequisite to interoperability

between different vendors. Our role as a measurements and standards organization is to make standards better by working with manufacturers to write correct, complete, and unambiguous standards; define conformance tests for use in the certification of implementations; perform public interoperability testing; and publicize standards activities.

We recognize that integration architectures can extend the reach of data exchange standards, but this program is not proposing new architectures. Rather, we are working within architectures currently in place and accepted by our industrial partners, and focusing on standards that fit within them. We continually assess which standards have the most potential to enable innovation, so that our contributions matter. The metric is the degree of industry support from both manufacturing end users and automation vendors. End users need to believe that the standard will make it easier for them to innovate, and need to commit to buy products that support the standard. Vendors need to believe that adherence of their products to the standard will raise demand for them, and that the cost of supporting the standard will be paid back over a reasonable time. The results are better standards and an American manufacturing environment 
in which it is easy to fit together best-in-class pieces to build a world-class product.

When companies believe that a standard is valuable to their businesses, they are willing to spend money to develop the standard. Often this is to ensure that their specific business interests are taken into account. However, the work that it takes to conduct test pilots, validate the standard and provide conformance tools provides benefits that accrue to all companies equally. No single company is willing to pay unequally for this. In some cases, companies pool their resources and set up an organization that is specifically funded to do the work, usually involving in-kind contributions from companies' technical staff. In other cases, public agencies like NIST or NASA take on this role, allocating some of their discretionary funding. However, the lack of any funded or in-kind industrial group is a warning that the proposed standard lacks value and does not merit a one-sided public investment. In the projects proposed here, we are working with well-organized industry groups with substantial in-kind investment and some funded membership.

\section{Results and Impact}

- Demonstrated integration of tolerance information from design with machining and inspection using the STEP-NC standard at Boeing's Renton WA facility in May 2009. A complex mold part was used as the basis for data exchange. STEP-NC data was easily visualized for operations and tools. Parts were machined at Boeing, Connecticut Center for Advanced Technology, KTH/Sweden and NIST, and brought to Mitutoyo/ Kirkland for inspection. The association between measured surfaces and the tools and operations that created them made it easy to see how tooling choices impacted quality; NIST results showed undercutting due to some tooling compromises.

- Tested the MTConnect machine tool data acquisition standard at Boeing's Auburn WA facility, writing a machine monitoring "dashboard" application that collects actual production figures for analysis and archiving. Tests were conduct during May 2009 on 60 machine tools from two vendors.

- NIST Co-sponsored in 2009 the $6^{\text {th }}$ and final public demonstration of interoperability using I++ DME ((a non-proprietary language for communicating with a coordinate measuring machine) at the Control Show

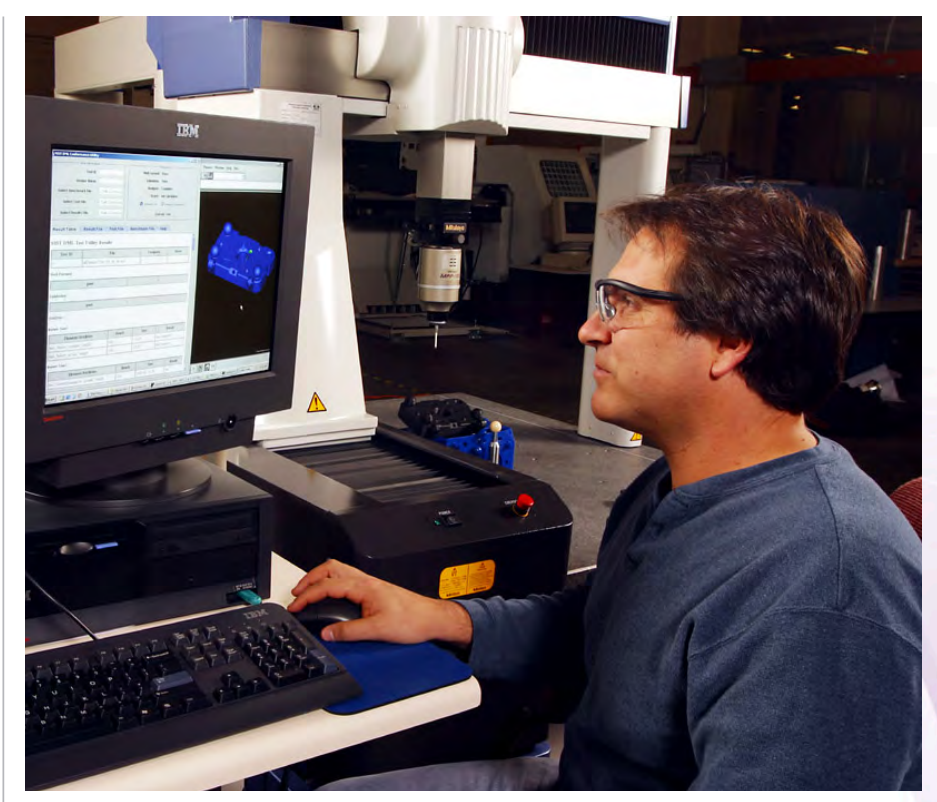

Testing standard interfaces for dimensional metrology equipment

in Stuttgart, Germany. The demo was co-sponsored by the International Association of CMM Manufacturers. Bill Rippey was the demo leader, technical coordinator, and demo results analyst. Seven execution software products and five Coordinate Measuring Machine (CMM) products implementing I++ DME measured three challenging parts with non-trivial operations (e.g., scanning, tool-changing, touch-probing), publicly demonstrating that interoperability is a commercial reality.

- Dane Barrager, Vice President of ASI DataMyte and chairman of the Automotive Industry Action Group (AIAG) Quality Measurement Data (QMD) Committee, noted that "the NIST QMD test suite greatly helped Statistical Process Control (SPC) software vendors to validate their QMD implementations. The validation was so correct and thorough that the vendors never passed any data [to other vendors] until the show Quality Expo Chicago 2007 began and everything worked correctly the first time." The QMD interoperability demonstration in 2007 was one of three such demonstrations, including one at Quality Expo Detroit in 2008. 
- NIST released version 2.1.5 1 of its Dimensional Measuring Interface Standard (DMIS) Conformance Test Suite, based on DMIS version 5.1. The DMIS Test Suite forms the basis for the recently-released DMIS Certification program of the DMSC (Dimensional Metrology Standards Consortium). Four major dimensional metrology corporations have applied for DMIS certification to test DMIS Conformance against the DMIS Standard.

\section{Publications}

Thomas Kramer and Frederick Proctor, "Feature-based Process Planning Based on STEP," and John Michaloski, Thomas Kramer, Frederick Proctor, Xun Xu, Sid Venkatesh and David Odendahl, "STEPNC++ - An Effective Tool for Feature-based CAM/CNC," in Advanced Design and Manufacturing Based on STEP, Springer Series in Advanced Manufacturing, Xun Xu, Xun and Andrew Nee (Editors), 2010, ISBN: 978-1-84882-738-7.

John Michaloski, Fred Proctor, Sid Venkatesh, Sydney Ly and Byeon-Eon Lee, "Quantifying the Performance of MT-Connect in a Distributed Manufacturing Environment," in Proceedings of the ASME International Design Engineering Technical Conferences \& Computers and Information in Engineering Conference, August 30 September 2, 2009, San Diego, CA USA.

CMM Quarterly (Special Edition on the DMSC), Sept 2009: John Horst, "Reduce Costs and Increase Quality with Information Exchange Standards for Manufacturing Quality"; William Rippey, "The Dimensional Markup Language Specification for Inspection Results Data," and "Update on DMIS Certification"; Thomas Kramer, "The NIST DMIS Test Suite Can Work for You".

1 http://www.isd.mel.nist.gov/projects/metrology_interoperability/ dmis_test_suite.htm

\section{Contact Information:}

Frederick M. Proctor

Group Leader, Control Systems Group

301-975-3425

frederick.proctor@nist.gov 


\section{Awards and Leadership}

\section{Board Membership}

\begin{tabular}{l|}
\hline Staff \\
\hline John Horst \\
John Horst \\
Leadership
\end{tabular}

\begin{tabular}{|c|c|}
\hline Staff & Leadership \\
\hline Fred Proctor & $\begin{array}{l}\text { Chair, ASME Computers and Information in Engineering } \\
\text { Division }\end{array}$ \\
\hline Fred Proctor & $\begin{array}{l}\text { Executive Committee of the ASME Computers and Infor- } \\
\text { mation in Engineering Division. }\end{array}$ \\
\hline Fred Proctor & $\begin{array}{l}\text { Conference Chair, } 2009 \text { ASME Computers in } \\
\text { Engineering conference, }\end{array}$ \\
\hline John Horst & $\begin{array}{l}\text { Chair, Dimensional Metrology Standards Consortium's } \\
\text { High-Level Measurements Process Planning (HIPP) } \\
\text { Committee }\end{array}$ \\
\hline John Horst & $\begin{array}{l}\text { Chair, DMSC Exchange of Quality Measurement Process } \\
\text { Plans (eQuiPP) Committee }\end{array}$ \\
\hline John Michaloski & $\begin{array}{l}\text { Chair, Open Modular Architecture Controller (OMAC) } \\
\text { Human-Machine Interface Working Group }\end{array}$ \\
\hline Bill Rippey & $\begin{array}{l}\text { Chair, Dimensional Metrology Standards Consortium's } \\
\text { Dimensional Markup Language (DML) Subcommittee }\end{array}$ \\
\hline John Horst & $\begin{array}{l}\text { Co-Chair, Automotive Industry Action Group (AIAG), } \\
\text { Metrology Interoperability Project Team Executive } \\
\text { Committee }\end{array}$ \\
\hline \multicolumn{2}{|l|}{ Awards } \\
\hline Staff & Award \\
\hline Bill Rippey & $\begin{array}{l}\text { Best Presentation, Metromeet 2009, "DMSC Offers New } \\
\text { DMIS Certification to Enable Interoperability" }\end{array}$ \\
\hline John Horst and Tom Kramer (Guest Researcher) & $\begin{array}{l}\text { Certificate of Achievement, Dimensional Metrology } \\
\text { Standards Consortium, April } 2008\end{array}$ \\
\hline Bill Rippey and John Horst & $\begin{array}{l}\text { AIAG Chairman's Award } 2009 \text { to NIST for work done on } \\
\text { QMD and DML specifications (and other projects) }\end{array}$ \\
\hline
\end{tabular}




\section{Intelligent Manufacturing Robotics and Automation Interoperability Standards Projects}

\section{Intelligent Manufacturing Interoperability Standards}

\section{Challenge}

$\mathrm{n}$ a global economy with competition from developing countries that have much lower labor rates, innovation is America's best hope to remain competitive. Innovation opens up new business opportunities and creates new ways to put people to work. The increasing pace of technological change and expectations of shorter time-tomarket for new products represent growing challenges for U.S. manufacturers. These pressures, combined with the push for distributed manufacturing facilities, create an urgent need for shop floor automation equipment that offer:

- Real-time integration between engineering, production, and business function, which may be geographically dispersed. Information and instructions need to be transmitted and acted upon without the delays that currently occur because of missing or misinterpreted data.

- Faster, more accurate, and more autonomous onboard planning, re-planning, and execution abilities that are driven by product and process models and respond to changes elsewhere in the manufacturing enterprise. Machine tools, robots, and coordinate measurement machines currently require extensive trial-and-error debugging whenever a new program is downloaded. This rework and tuning by human operators results from the hard-coded nature of the programs, which cannot adapt to individual differences in each piece of equipment. The challenge is to provide the necessary information about the part design (features, tolerances, etc.) as well as the machine's own characteristics so that it can automatically adjust for its own motion errors and other variations.

- Interoperability of products from diverse vendors. Manufacturing enterprises must be able to buy the best equipment for their needs and to outsource production of various components. For these options to be economically viable, data must be reliably transferred among machine tools, robots, and coordinate measurement machines. U.S. manufacturers must be able to buy equipment with confidence that it will integrate with the rest of their enterprise.
The problem is hard because the factory floors is where engineers from many different disciplines come together in a tangle of incompatible equipment, and are tasked with making things work on tight schedules. The different disciplines have disparate perspectives on the information that is necessary; hence it is a tremendous challenge to determine which data are needed, and how to express them unambiguously and efficiently.

\section{Objective}

elp U.S. manufacturers reduce the cost of innovation, by removing the barriers they face when bringing together best-in-class automation solutions to build world-class products.

\section{Technical Plan}

Ne are working to achieve a vision of the seamless transfer of "smarter" data. This is an essential prerequisite not only for reliable transfer of information between equipment controllers, but also for providing enough richness in the information to enable the shop floor equipment to make in situ decisions when new programs are received and when conditions change. This vision of seamless smart data transfer presents business and technical challenges. To address the business challenge, which is rooted in resistance to standards, ISD involves vendors and end users in the definition of requirements, standards, and validation. The technical challenges center on the definition of the data models and interface protocols themselves: are they complete, efficient, unambiguous, and correct? ISD is addressing the technical challenges by developing measures of the correctness of interface language specifications and by implementing standards validation tools and processes.

We are focusing on two key segments of the industrial automation market: metrology equipment and machine tools. Metrology equipment standards cover interfaces between computer aided-design (CAD) and product life cycle management software, inspection process planning software, inspection execution software, coordinate measuring machines, and reporting and analysis software. For machine tools, ISD has been focusing on the ISO 10303 (Standard for the Exchange of Product Model data, "STEP") standards for product date exchange, specifically the STEP-Numeric Control (NC) standard for programming machine tools; and two standards for extracting real-time production information from machining equipment: OPC and MTConnect. 


\section{Quality Information Interoperability}

\section{Challenge}

T he information incompatibility problem in manufacturing quality is costly to everyone: end users, software vendors, and equipment vendors. Information exchange standards eliminate these costs, as long as there is strong end user leadership and strong vendor participation.

\section{Objective}

nable verifiably correct, complete, unambiguous, and timely information exchange standards and implementations of those standards. This enables greater interoperability and collaboration using standard information exchange languages. This further enables cost savings and greater agility to the entire enterprise. This finally fosters technology and product innovation.

\section{Technical Plan}

T he scope of this industry-led effort is on the exchange of information between quality measurement-related activities such as design, planning, measurement, analysis, and process feedback, including feedback of results to manufacturing production. NIST's role is to 1) help industry define correct, complete, and unambiguous interface standards, 2) design, build, and maintain conformance tests, 3) help industry design and lead public interoperability tests, and 4) act as standards consultants and leaders in appropriate industry standards organizations.

\section{Results and Impacts}

- NIST and the International Association of CMM Manufacturers co-sponsored in 2009 the $6^{\text {th }}$ and final public demonstration of interoperability using I++ DME (a non-proprietary language for communicating with a coordinate measuring machine) at the Control Show in Stuttgart, Germany. Seven execution software products and five CMM products implementing I++ DME measured three challenging parts with non-trivial operations (e.g., scanning, tool-changing, touch-probing), publicly demonstrating that interoperability is a commercial reality. As a result of these efforts metrology systems users are now using I++ DME compliant products in their production facilities worldwide.
- NIST released version 2.1.5 of its DMIS Conformance Test Suite, based on DMIS version 5.1. The DMIS Test Suite forms the basis for the recently-released DMIS Certification program of the DMSC (Dimensional Metrology Standards Consortium). Four major dimensional metrology corporations have applied for DMIS certification. Certification will help bring about greater levels of interoperability.

- At the 2009 Collaboration and Interoperability Conference $(\mathrm{CIC})$, a non-proprietary conference on design information interoperability, ISD organized and conducted a session entitled, "CAD Manufacturing Interoperability," and moderated a panel discussion entitled, "I Want My PMI! (Product Manufacturing Information) But How Do I Use It and Benefit From It?" Requirements from users for Computer aided Design (CAD) + PMl information accessible to downstream applications in non-proprietary formats have been increasing over the last several years.

- With MEL support, the DMIS standard progressed from versions 5.1 and 5.2, incorporating information required by important new technologies. DMIS 5.2 is now an ANSI standard and should become an ISO standard in 2010.

- Moved DML (Dimensional Mark-up Language) effort moved from AIAG to to a DMSC subcommittee, where it continues to thrive, with the strong hope for ISO standardization sometime in 2011.

\section{Customers and Collaborators}

Ford Motor Company, Honeywell FMT, General Electric Aviation, Deere and Co., Chrysler LLC, Daimler, Audi, Volvo, Volkswagen, Peugeot, Timkin, Caterpillar, BMW, International Association of Coordinate Measuring Machine Manufacturers (IA.CMM), Automotive Industry Action Group (AIAG), Society of Manufacturing Engineers (SME), ISO STEP, Inspection-plus-plus Group (I++), Longview Associates, Dimensional Metrology Standards Consortium (DMSC), Mitutoyo, Wenzel, Metromec, Hexagon Wilcox, Hexagon Brown \& Sharp, Sheffield, Helmel Engineering, Dassault, Renishaw, Faro, Applied Precision, Inc., Zeiss, Hexagon Messtechnik Wetzlar, Wenzel Xspect Solutions, Statpoint, Minitab, New River Kinematics, QMC, Infinity QS, Prolink, Nikon Metrology, Metrosage, Rolls Royce 


\section{Next Generation Machine Tool Control}

\section{Challenge:}

he challenge is to make possible the development

of faster, more accurate, and more autonomous machine tools that are driven by product and process models and are responsive to the manufacturing enterprise. Challenges to realizing the vision of smart data for smart machines are the resistance of vendors to standardization (a business challenge) and the development of the data models themselves (a technical challenge).

\section{Objective:}

upport industry groups by validating data exchange standards. Paraphrasing BASF, "We don't make the standards you use, we make the standards you use better."

\section{Technical Plan:}

o address the business challenge, we realize that vendors and end users must be involved in the development of standards and pull for their success. Looking at the extent of vendor- and end-user involvement and the potential markets for U.S. companies, MEL prioritizes candidate standards and selects those that have the most potential. Along the way, it is essential to continually assess these markers to decide whether to continue participating. STEP-NC, OPC and MTConnect have shown growing involvement from vendors and end users (aerospace and automotive) with more impressive demonstrations each year.

\section{Results and Impacts:}

- Demonstrated integration of tolerance information from design with machining and inspection using the STEP-NC standard at Boeing's Renton WA facility in May 2009. A complex mold part was used as the basis for data exchange. STEP-NC data was easily visualized for operations and tools. Parts were machined at Boeing, Connecticut Center for Advanced Technology, KTH/Sweden and NIST, and brought to Mitutoyo/Kirkland for inspection.

- Tested the MTConnect machine tool data acquisition standard at Boeing's Auburn WA facility by writing a machine monitoring "dashboard" application that collects actual production figures for analysis and archiving. Tests were conducting during May 2009 on 60 machine tools from two vendors.

\section{Customers and Collaborators}

Boeing, General Motors, GE/Fanuc, Lockheed-Martin, Mastercam, Okuma USA, Sandvik Tooling, Siemens PLM Software, STEP Tools Inc., The Association for Manufacturing Technology (AMT), The International Organization for Standardization (ISO), The Open Modular Architecture Controller (OMAC) Users Group 


\section{Measurement Science for Intelligent Manufacturing Robotics and Automation}

Seamless cooperation between humans and robots in manufacturing systems can combine the precision of machines with the dexterity and fault tolerance of peopleproviding a revolution in creativity and speed on the factory floor. But creating such systems will require huge innovations for automated equipment, such as robots and machine tools, to intelligently carry out production plans and easily adapt them in response to changes in material, process, equipment, or surroundings. This project seeks new measurement science on all scales, from the macro to the nano, to explore how intelligent systems might do anything from perceiving movement in their vicinity to meeting production goals to protecting nearby humans. Such flexible systems will improve safety and make it easier and quicker to build new products.

\section{Staff}

10 FTE NIST staff

5 Guest Researchers

15 total FTEs

\section{Challenge}

\footnotetext{
anufacturers anticipate a future in which humans $\checkmark$ will work side by side on the shop floor with versatile and adaptable robots and automation systems. To realize this vision, equipment will need to perceive the geometry, location, color, features, and movements of items and people in its vicinity and have an intrinsic ability to adjust for variations in parts, environment, and other conditions, rather than relying on fixed automation and costly infrastructure. This must be achieved in both macro-scale manufacturing and in the emerging microand nano-scale manufacturing industries. The long-term challenges include developing the performance measurement science to characterize constituent components of intelligent manufacturing systems, defining the target performance goals, and measuring how well a component or overall system meets the goals.
}

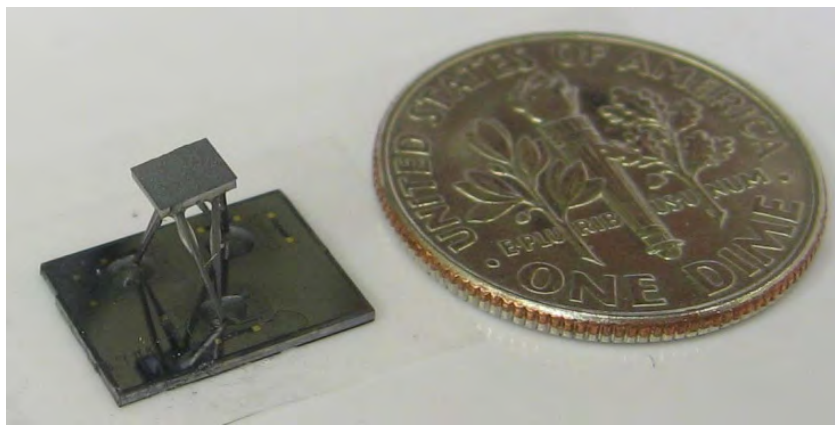

A micro-sized six degree of freedom manipulator.

\section{Technical Strategies}
ost current factory machinery is painstakingly $\checkmark$ programmed to blindly repeat a single task over and over until told to stop, so that new production lines require major retooling. In addition, machinery - es- pecially industrial robots - is walled off from human workers because it is deemed unsafe to function in their presence. To enable safer, more flexible manufacturing environments, the program is working with industry, including airplane and car manufacturers, to provide the measurements, standards, and performance evaluation methods necessary to make robots more adaptable. 
One of the most crucial necessities is providing robots with the ability to accurately perceive their surroundings. A machine that can perceive complex activity in its vicinity can make adjustments on the fly for changes in materials, equipment, or surroundings. The Measurement Science Program develops robust, quantitative tests to ensure that advanced perception systems- from the physical sensors themselves to the software programs that run them-accurately sense the three-dimensional world of moving parts in their vicinity, allowing them to alter plans when they detect an anomaly in production.

As well as providing flexibility on the shop floor, sensors can be programmed to detect and avert situations in which humans could be harmed. NIST works with the standards community to define methods and evaluation tests by which intelligent automation, including robots and automatic guided vehicles can be "certified safe," ushering in an era of human-robot cooperation that can provide U.S. industry with the high-quality, high-precision manufacturing methods it needs to maintain global competitiveness.

Furthermore, the Program provides industries with the necessary standards, performance metrics, and infrastructure technology to support the use of semi-autonomous and autonomous manipulators and vehicles, control system architectures, and advanced measurement tools and methods that enable their use in static and dynamic assembly operations and material handling for manufacturing. It accomplishes this through both real-world tests on a shop-floor test bed that includes an industrial robot and an autonomous vehicle, and through a physics-based simulation environment that merges seamlessly with the real world but provides adaptable, safe, and repeatable environment where manufacturing automation and robot performance standards can be safely developed and rapidly tested.

The Program also addresses standards for the newest branch of automation: nanorobotics. Nanorobotics encompasses both minuscule machines and large machines that fabricate minuscule objects. The program works to develop control and positioning systems for nanoscale measurements and manipulation and to enable interfaces to the macro-scale world. Standards are needed to determine the accuracy of such machines-did it really insert a new part exactly 10 nanometers to the left as expected? - as well as to evaluate the precision with which tiny devices are built.

\section{Results and Impacts}

- The Virtual Manufacturing Automation Competition was successfully run at the IEEE International Conference on Robotics and Automation. The competition featured autonomous unit loaders performing intrafactory transport of goods. These competitions foster innovation and provide an ideal venue for testing performance measures.

- As a result of sensor measurements conducted under the program, wording in the ANSI B56.5 standard for Guided Industrial Vehicles and Automated Functions of Manned Industrial Vehicles was modified to allow industrial vehicles to utilize non-contact safety sensors.
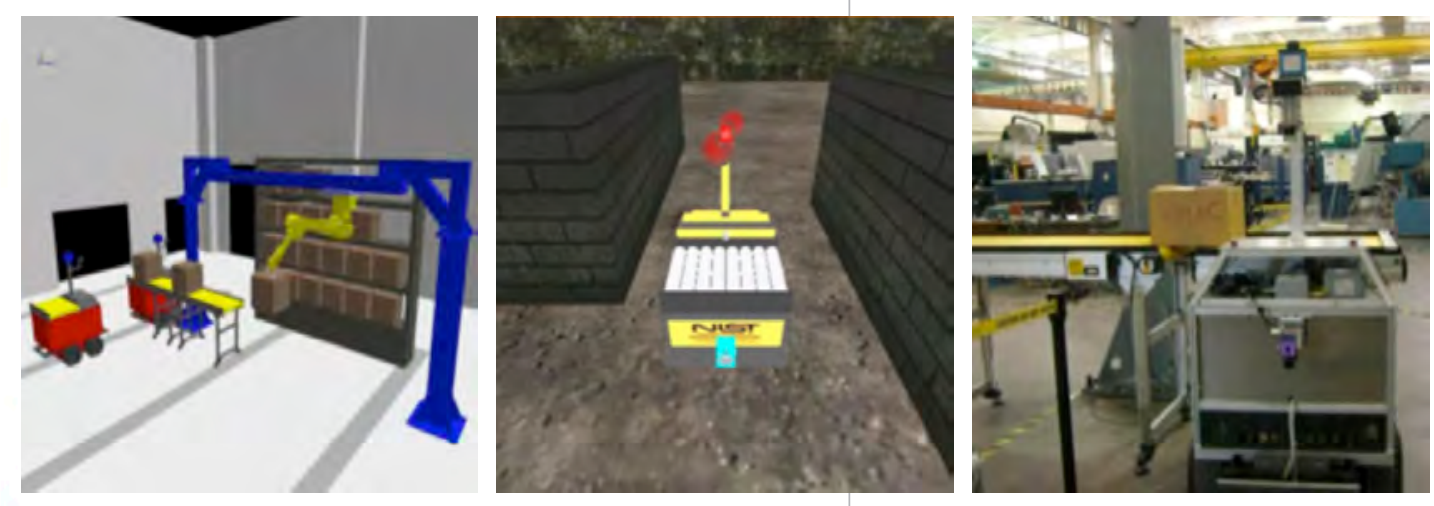

The real-virtual environment: (left) Virtual Test Bed, (center) Virtual Atuonomous Guided Vehicle (AGV), (right) Real Test Bed. 
- Two patent disclosures have been submitted for nanoscale measurements. An embedded Micro-ElectroMechanical Systems (MEMS) nano-displacement measurement sensor with accuracy between $8 \mathrm{~nm}$ and $9 \mathrm{~nm}$ was invented and constructed, and a dynamic nano-rheology sensor was developed (with NIST's Material Sciences and Engineering Laboratory) to measure the dynamic rheology properties of nanoscale material solutions. These instruments enable high precision dynamic measurements of displacement and chemical properties of great interest for pharmaceuticals and custom materials development.

- Members of the program participate in a new USCAR ${ }^{1}$ project, titled "Advanced Perception and System for Safe and Dynamic Human Robot Interaction". The project is evaluating the possibility of reducing the need for fencing and other space-consuming safety equipment by the use of advanced perception and human detection and tracking. This could have a large impact on factory floor layouts, reduce the resources needed for safety equipment, and reduce the entry costs for small and medium-sized businesses that want to employ robots

\section{Publications}

B. Balaguer, S. Balakirsky, S. Carpin, A. Visser, "Evaluating maps Produced by Urban Search and Rescue Robots: Lessons Learned from RoboCup." Autonomous Robots, Springer US, 2009, vol. 27, no. 4, pp. 449-464

R. Eastman, E. Messina, T. Hong, H. Huang, M. Shneier, J. Shi, and J. Wells, "Dynamic Perception Workshop Report: Requirements and Standards for Advanced Manufacturing," NIST-IR 7664, 2010.

G.F. Christopher, N. G. Dagalakis, S.D. Hudson, K.B. Migler, "MEMS Parallel-Plate Rheometer for Small Amplitude Oscillatory Shear Micro Rheology Measurements," Society of Rheology, $81^{\text {st }}$ Annual Meeting, Madison, WI, October 18-22, 2009.

\section{Contact Information}

Michael Shneier, Program Manager

(301) 975-3421

michael.shneier@nist.gov

http://www.nist.gov/mel/isd/si/msimra.cfm.

1 USCAR is an umbrella organization of DaimlerChrysler, Ford and General Motors, formed to conduct cooperative, pre-competitive research. 


\section{Awards and Recognition}

\begin{tabular}{|c|c|}
\hline Staff & Leadership \\
\hline Roger Bostelman & $\begin{array}{l}\text { - Sub-Committee member - "American National Standards Institute/Industrial Truck Stan- } \\
\text { dards Development Foundation (ANSI/ITSDF) B56.5 Safety Standard for Driverless, Auto- } \\
\text { matic Guided Industrial Vehicles and Automated Functions of Manned Industrial Vehicles" }\end{array}$ \\
\hline Jason Gorman & $\begin{array}{l}\text { - Special Sessions Chair, IEEE International Conference on Automation Science and Engi- } \\
\text { neering, Toronto, Canada, } 2010 \\
\text { - Co-Organizer, Symposium on Measurement and Control of Micro- and Nanosystems, } \\
\text { Conference on Micro- and Nanosystems, ASME IDETC, Montreal, Canada, } 2010 . \\
\text { - Invited presentation: "Microrobotic Technologies," Unmanned Systems Summit 2009, } \\
\text { National Harbor, MD. } \\
\text { - Invited presentation: "Precision MEMS for Nanoinstrumentation," AVS Regional Meeting, } \\
\text { Gaithersburg, MD, 2009. } \\
\text { - Invited presentation: "Precision MEMS/NEMS in Nanoinstrumentation," nanotxUSA'08 } \\
\text { International Nanotechnology Conference, Dallas, TX. }\end{array}$ \\
\hline Tsai Hong & $\begin{array}{l}\text { - Chair Workshop on Dynamic Perception Workshop: Requirements and Standards for } \\
\text { Advanced Manufacturing at the RIA Conference in June, } 2009 . \\
\text { - Co-chair of session on Performance metrics for Perception in Intelligent Manufacturing. } \\
\text { - Co-chair of session on Ground Truth and Testbeds for Performance Testing in PerMIS } 2009 . \\
\text { - Invited presentation at the department of Mechanical and Aerospace Engineering } \\
\text { of GW University. }\end{array}$ \\
\hline Nicholas Dagalakis & $\begin{array}{l}\text { - Became a Senior member of the IEEE } \\
\text { - Recognized by US Senate Special Committee on Aging } 2008 \\
\text { - NIST Bronze Medal Award } \\
\text { - Member of the Robotic Industries Association (RIA) } 15.06 \text { committee on industrial robot } \\
\text { safety standards. }\end{array}$ \\
\hline Stephen Balakirsky & $\begin{array}{l}\text { - Best paper award for: S. Balakirsky, F. Proctor, C. Scrapper, T. Kramer. A Mobile Robot } \\
\text { Control Framework: From Simulation to Reality. 2008. International Conference on Simu- } \\
\text { lation, Modeling, and Programming for Autonomous Robots (SIMPAR 2008). 11-3-2008. } \\
\text { - S. Balakirsky, F. Proctor, J. Falco, Government Open Source Conference (GOSCON) } \\
\text { Engaging Citizens Award. } \\
\text { - Executive committee of RoboCup International. } \\
\text { - Program Co-chair for America for Simulation, Modeling, and Programming, for } \\
\text { Autonomous Robots (SIMPAR) } \\
\text { - } 2010 \text { Co-organizer for ICRA } 2010 \text { Workshop “ICRA } 2009 \text { Robot Challenge Event: Out- } \\
\text { comes and Future Directions" } \\
\text { - Co-organizer for IROS } 2009 \text { Workshop "Robots, Games, and Research: Success stories in } \\
\text { USARSim" } \\
\text { - Invited talk to MIT Laboratory for Manufacturing and Productivity. }\end{array}$ \\
\hline
\end{tabular}


Measurement Science for Intelligent Manufacturing Robotics and Automation Projects

\section{Metrology and Standards for Advanced Perception Systems for Intelligent Manufacturing}

\section{Challenge/Problem Addressed}

$T^{n}$ he challenge is to enable wide use of advanced perception systems by intelligent automated manufacturing applications in unstructured environments. Despite substantial advances in sensors and algorithms over the past decade, there remains a lack of reliable, well-validated commercial perception systems. This is a major bottleneck in the development and deployment of flexible manufacturing systems that can use intelligent perception to sense and react in real-time.

In the National Council for Advanced Manufacturing (NACFAM) ${ }^{2}$ study and the Computing Community Consortium's Robotics Roadmap ${ }^{3}$, advanced perception systems and sensors were identified as technology enablers that could shift manufacturing to more flexible, adaptable and safe automated systems. Automated manufacturing is starting to rely heavily on sensors as it moves into less structured environments and deals with a greater variety of tasks and components. Sensors are needed to locate parts, identify them and determine their properties, and manipulate them robustly in threedimensional space. Currently, very few manufacturing applications involve any sensing at all, and those that do usually involve simple measurements under highly restricted conditions. These limitations incur costs in terms of efficiency, restricted work cell layout, and the need for expensive work cell components such as fixturing and handling equipment. This is one of the major factors that have prevented the widespread adoption of robotics and automated manufacturing by small and medium sized businesses.

2 Report on industry Views Towards: Categories of innovative and Potentially Disruptive Advanced Manufacturing Technologies." NACFAM (2005).

3 "A Roadmap for U.S. Robotics: From Internet to Robotics," CCC and CRA (NSF-funded), 2009, http://www.us-robotics.us/

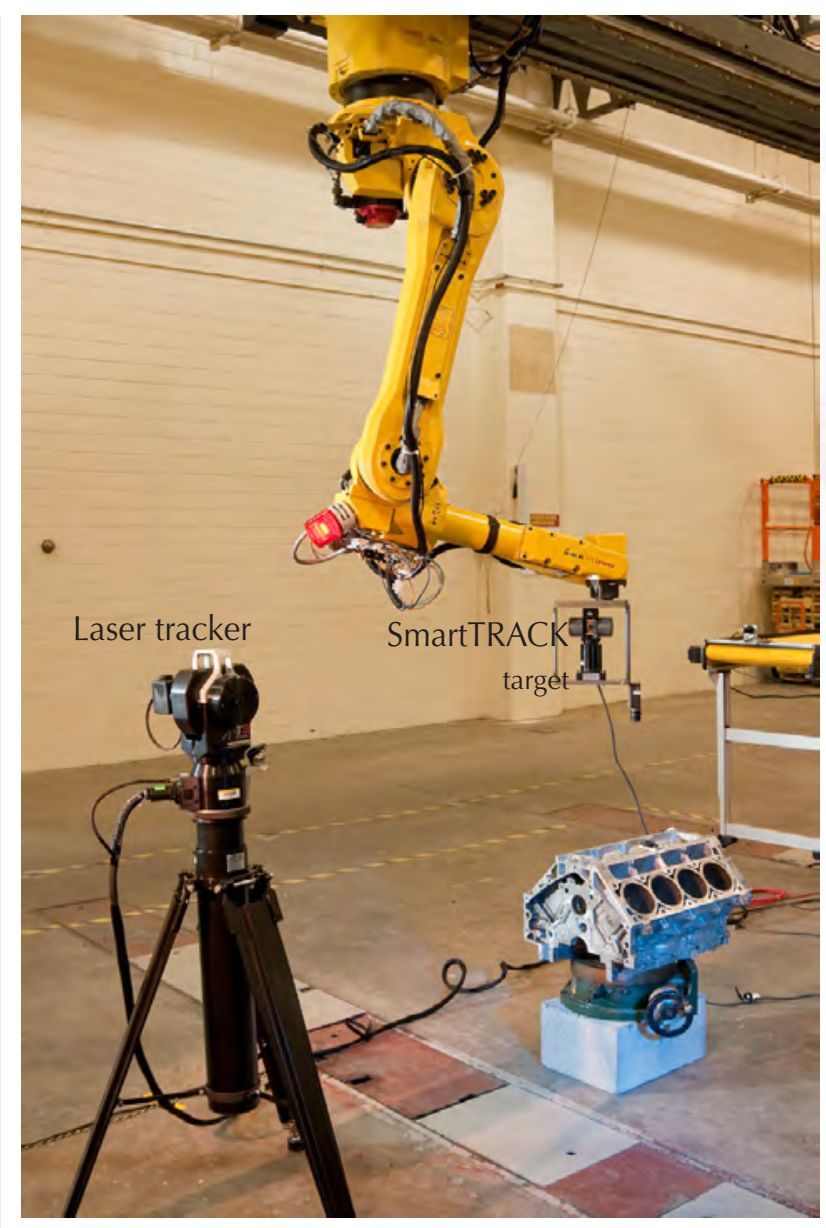

View of the laser tracker and SmartTRACK target in the test bed, measuring a calibration target

Barriers to the introduction of sensors have typically included the high costs of developing custom applications, the lack of guarantees that the sensor systems will work consistently enough for a production environment, and the fact that, in the automation community, sophisticated sensing is still in its infancy and users have little experience in using it in their applications. The project addresses these barriers by defining performance measures and metrics for sensor systems and promoting standards that help vendors describe their products in a uniform way and help users select the right products for their applications. The project also addresses another current barrier to more widespread use of advanced automation: the need to separate robots and automation equipment from people working in the same work area. Sensors will have a major part in enabling people and robots to work together safely. The project addresses the performance metrics and standards that are needed to ensure that sensors provide the necessary levels of safety and reliability to enable human-robot collaboration. 


\section{Objectives}

evelop quantitative, reproducible test methods to evaluate the robustness, accuracy and performance characteristics of advanced perception systems for general assembly, human robot collaboration and other macro scale manufacturing operations.

- Develop reliable and accurate procedures for internal and external calibration of 2D, 3D and six degree of freedom (6DOF) sensors, so as to enable cross-sensor comparison and evaluation.

- Develop metrology and test methods to evaluate the accuracy and performance of 3D and 6DOF sensors under dynamic conditions.

\section{Technical accomplishments}

- Established and validated a test bed for evaluating six degrees of freedom (6DOF) perception sensing. The test bed will help develop standardized evaluation procedures that will assist industry and academia to establish clear performance metrics for research algorithms and help manufacturers measure and understand the motion of conveyances, parts and assemblies.

- Developed performance evaluation methods and procedures for pose determination algorithms in static and dynamic environments and published the results in PerMIS 2008 and 2009. Such methods will enable manufacturers to compare pose measurement systems in a standardized way.

- Conducted a performance evaluation of the external calibration of a camera and laser range finder. Used the procedure to validate the calibration of a suite of sensors for General Dynamics Robotic Systems.

- Conducted a Dynamic Perception Measurement for Autonomous Manufacturing Workshop at the RIA International Robots, Vision \& Motion Control Show in June, 2009. The workshop produced sensor requirements and test scenarios useful for evaluating perception systems in next generation manufacturing, which are documented in a workshop report.

- Organized a special workshop session on Performance Metrics for Perception in Intelligent Manufacturing as part of the PerMIS workshop in 2008. Published a book chapter on this session. The workshop produced an initial roadmap for measurement of perception systems in next generation manufacturing.
- Designed and constructed a set of modular manufacturing-part artifacts for evaluating perception systems. Incorporated 6DOF sensing into a comprehensive robotic ontology and developed a first draft of a proposed standard terminology for 6DOF sensing. The standardized artifacts and ontology will facilitate understanding of and research in dynamic perception.

\section{Planned Future Accomplishments}

- Continue to develop standard procedures and metrics for 6DOF sensors by developing mathematical techniques for calibration and performance metrics; prototyping a generic data collection system to foster system evaluations; establishing reference standards for 6DOF sensors, and validating these standards and metrics using commercial and academic vision systems.

- Continue to establish a standards group for 6DOF performance metrology and standards that will include developing consensus-based performance test methods and metrics to evaluate advanced perception systems in dynamic and unstructured environments and developing a comprehensive kit of standardized parts for these test methods.

- Collaborate with industry and academic groups including work with USCAR on their fenceless robotics project, which involves performance evaluation of perception systems for detecting humans. If successful, this work will greatly increase the flexibility and decrease the costs of work cells.

- Work with Middlebury College on metrics and ground truth for evaluation of multi-view stereo, which should lead to standards and performance measures for determining the positions and orientations of parts using stereo.

\section{Customers and Collaborators}

USCAR, GM, Ford, Chrysler, Automated Precision, Inc., Braintech, Inc., JAI Inc., Loyola University, Purdue University, Middlebury College, Army Research Laboratory, General Dynamics Robotic Systems. 


\section{Next Generation Robot Safety}

\section{Challenge/Problem Addressed}

ollaborative human-robot interaction holds promise for a growing number of applications. Robots can work closely with humans to reduce the frequency of repetitive tasks, to reduce the time humans take to perform tasks, and to reduce human fatigue and injuries. Current safety standards limit human-robot interaction, require significant portions of valuable manufacturing production floor space for fences and other protective devices, and involve significant expenditure for protective equipment. The typical unit cost for industrial robots ranges from approximately $\$ 30 \mathrm{~K}$ to $\$ 50 \mathrm{~K}$, but the cost of protective equipment frequently doubles the cost of the robotic units themselves. To enable human-robot interactions requires new, inexpensive and reliable safety technologies and relevant, up-to-date safety standards. Advances in technology and standards will lead to reductions in the cost of manufacturing and an increase in U.S. competiveness.

\section{Objectives}

- Install safety system on testbed robot to provide protection to NIST and outside researchers from injuries caused by test bed robot and to gain experience with current industrial safety standards.

- Assist standards organization in developing humanrobot collaborative safety standards and test procedures.

- Work with manufacturers to develop safety requirements and technologies to enable humans and robots to interact in assembly operations.

- Develop a robot test bed for verifying that proposed safety standards provide required protections.

\section{Technical accomplishments}

- Completed installation of a safety system in the NIST robot test bed, in accordance with the current standard. Carried out and documented several verification tests and presented them at a Robotics Industries Association (RIA) robot safety meeting.

- Participated in the RIA/ISO robot safety standard effort to develop a technical specification for human-robot collaboration. Contributed significantly to all the RIA and ISO meetings. Assessed human-robot collaborative safety features for new robots at a number of robot manufacturers to determine the gaps between what is currently offered and what is needed for the new standard (ISO 10281-2).

- Drafted a technical specification (TS) for speed and position monitoring (SPM) of robots at RIA's request. Using SPM, robot systems are designed to maintain a safe distance between the operator and the robot. Sent a draft specification to RIA for submission to the ISO 10218 (Robots for Industrial Environments) committee. Completion of the ISO 10218-2 safety standard requires completion of the TS. A prototype SPM safety module was implemented on the robot test bed and tests were begun to evaluate SPM functionality and to validate the TS. Completion of the TS will allow industry to proceed with development of safe human-robot collaborative applications and products.

\section{Planned Future Accomplishments}

- Contribute to the technical specification for ISO 10218-2 robot safety standard through further work on speed and position monitoring. Material proposed by other ISO members will be reviewed and perhaps tried out in the test bed. Use the robot test bed to verify SPM scenarios and to develop SPM test and verification procedures. It will also serve as an environment for measuring SPM performance.

- Write a report covering the state of the art in human sensing and robot safety features to assist the USCAR project team in their feasibility study.

\section{Customers and Collaborators}

Robotics Industry Association 15.06 committee on industrial robot safety; ISO 10218-2 Robot Safety Committee; USCAR, GM, Ford and Chrysler 


\section{Mobility and Manipulation Performance Metrics and Standards Project}

\section{Challenge/Problem Addressed}

he project provides industries with standards, per-
formance metrics, and infrastructure technology to support the use of semi-autonomous and autonomous manipulators and vehicles, control system architectures, vehicle safety systems, and measurement tools and methods in static and dynamic assembly and material handling for manufacturing. Collaborations with key robot arm and automated guided vehicle (AGV) developers and user partners advance the state of autonomous robotic technology through robot arms, mobile robots, non-contact safety sensors and open, modular control system architectures that enable broader use of advanced perception, autonomous navigation, manipulation, and parts and material handling techniques in the automotive, aerospace, and other industries.

Automobile, heavy equipment, and many other manufacturers can benefit from safe human-robot collaboration where vehicles and humans are expected to operate in robot work cells safely. To understand current and future automated equipment performance capabilities, the program is developing a manufacturing test bed and invites industry to provide application scenarios for prototyping. The manufacturing test bed also provides an integration environment for the other projects within the program.

The test bed is used to measure performance of automated assembly scenarios and to develop performance standards for manufacturing equipment and tools used in this and other advanced human-robot collaborative applications.

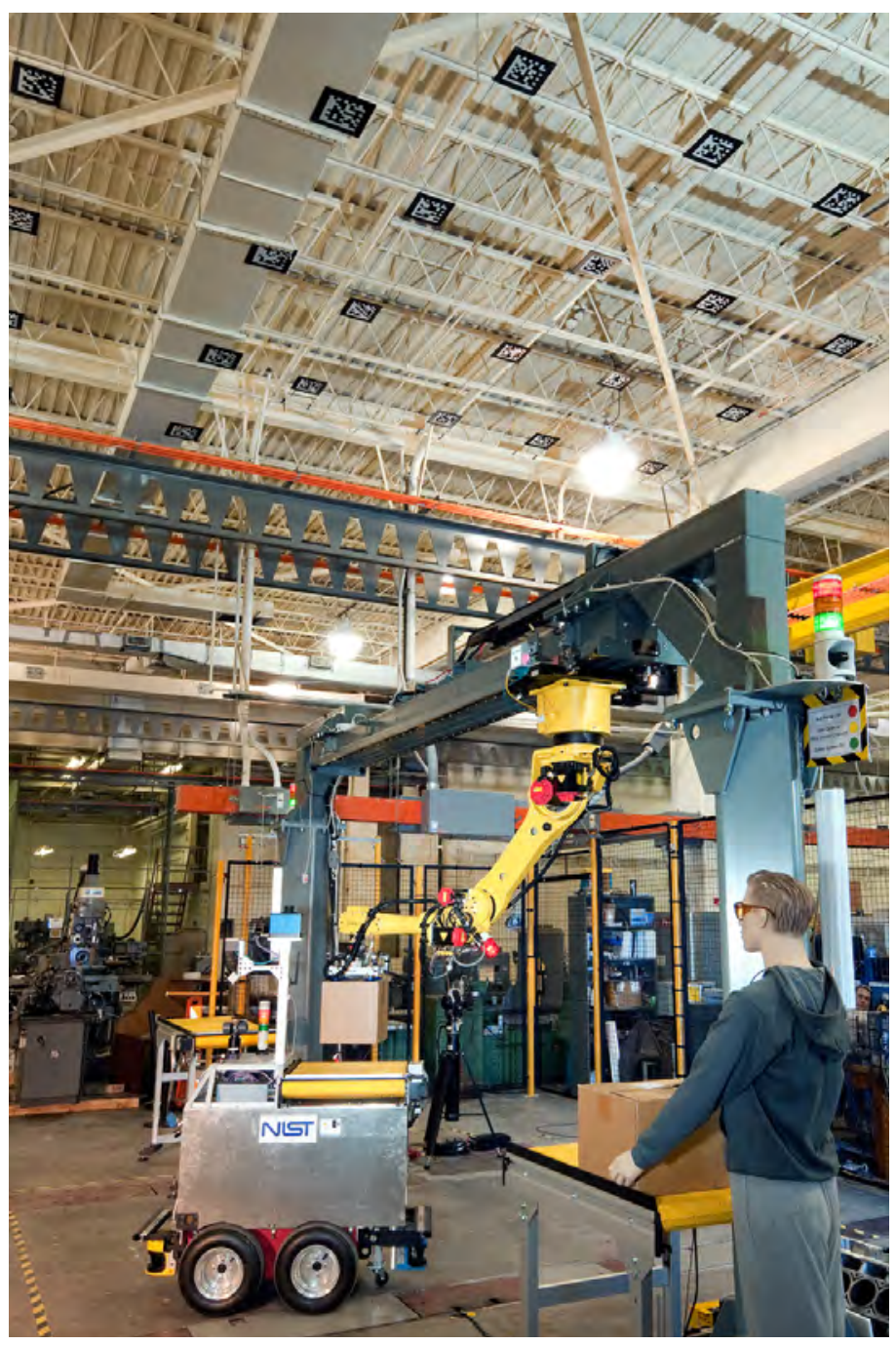

\section{Objective(s)}

- Design and develop an autonomous assembly and measurement test bed to provide an environment similar to that in industry. The test bed provides the infrastructure for developing performance measures and standards to be used in manufacturing.

- Develop performance measurement methods for autonomous assembly and measure performance of automated assembly methods in the test bed. This will lead to new standards and ways of evaluating products in real-world environments.

- Lead industrial robot and vehicle standards committees by developing performance test methods and data. These standards will encourage the next-generation in advanced human-robot collaborative applications. 


\section{Accomplishments}

- Designed, procured, installed and tested equipment for the robot test bed for the autonomous assembly performance measurements and standards efforts.

- Developed autonomous assembly scenarios showing AGV and robot collaboration to illustrate to AGV and robot vendors the potential of such interactions and the barriers to their realization.

- Designed and tested the AGV with safety systems based on ANSI B56.5 standard. This standard defines safety requirements for powered, unmanned automatic guided industrial vehicles. Performed advanced sensor measurements in support of ANSI B56.5 and developed text to improve the standard to now allow industrial vehicles to utilize non-contact safety sensors.

- Led a Special Session on Performance Measurements Towards Improved Forklift Safety, PerMIS 2009. This led to a lot of industry interest, including three articles written by trade journals describing proposed improvements.

- Developed a novel safety sensor system design for AGVs and forklifts and measured the performance of advanced 3D imagers that could be used to provide all-round safety coverage for these vehicles.

- Converted a standard AGV into a safer, more generic vehicle through low level electronics replacement and enhancements to provide proof-of-concept that AGVs can be used safely with humans in close proximity.

\section{Planned Future Accomplishments}

- Implement manufacturing assembly scenarios based on direct industry interaction to study manufacturing challenges and leading to improved performance and standards.

- Measure the performance of advanced 3D imagers for use in a robot work cell for safe and effective human/ robot/vehicle collaboration.

- Work with standards organizations to provide draft test methods and results from experiments with advanced sensing and control technologies for safe autonomous assembly, material transport, logistics, and other shop floor operations. This includes evaluation of auxiliary safety sensors for fork lifts (with or without human drivers).
- Analyze and work with international standards organizations (ISO 13482) to leverage common technologies between medical/healthcare equipment and manufacturing equipment. Examples of such potential crossover technology include prosthetic arms vs. industrial robots and autonomous chairs vs. AGV's and forklifts.

\section{Customers/Collaborators}

USCAR: GM, Ford, Chrysler;SAE, Boeing, Northrop Grumman, US Army, Navy, Air Force, Robotics Industries Association (RIA), Material Handling Industries Association (MHIA), ISO, AGV manufacturers and users, Forklift manufacturers and users, Sensor manufacturers and users 


\section{Performance Simulation (PerfSim)}

\section{Challenge/Problem Addressed}

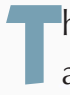
he challenge is to provide industry with an adaptable, safe, and repeatable environment where manufacturing automation and robot performance standards can be developed and rapidly tested. The project will develop both a standardized simulation interface and a validation framework that will allow researchers to know when the use of a particular simulation is appropriate. The use of simulation is expected to accelerate the introduction of more advanced robotics for manufacturing by allowing for greater flexibility and speed in experimentation and validation.

Standardized simulation interfaces allow researchers and developers to test against, and participate in, the development of evolving performance standards without costly infrastructure requirements. However, these tests are only as good as the simulation is valid. The project uses a hybrid real/virtual system and modular architecture that allows for the seamless integration of real hardware components and algorithms with virtual reality. The framework makes it possible to validate simulated components as well as create novel notional components. For example, simulated avatars will be able to test robot/ human safety standards, repeatable simulated system faults test reliability and fault tolerance, and simulated sensor outputs allow for the analysis of potential system improvements through the incorporation of currently unattainable levels of sensor performance.

\section{Objectives}

- Develop task specific validation techniques for simulation systems. By determining simulation fidelity requirements for a range of common simulation applications, developers will better understand when the use of simulation is a viable alternative to physical testing and development.

- Develop a framework that allows for the seamless transition of virtual performance metrics to realizable physical artifacts that remain coupled to the simulation environment. Use of test environments that combine real and virtual elements will allow for performance quantification of systems that are in the vicinity of humans or in collaboration with them without posing any risk to human participants.

- Support the use of simulation systems in international competitions. These competitions foster innovation and provide a high-visibility test environment for emerging performance metrics. 


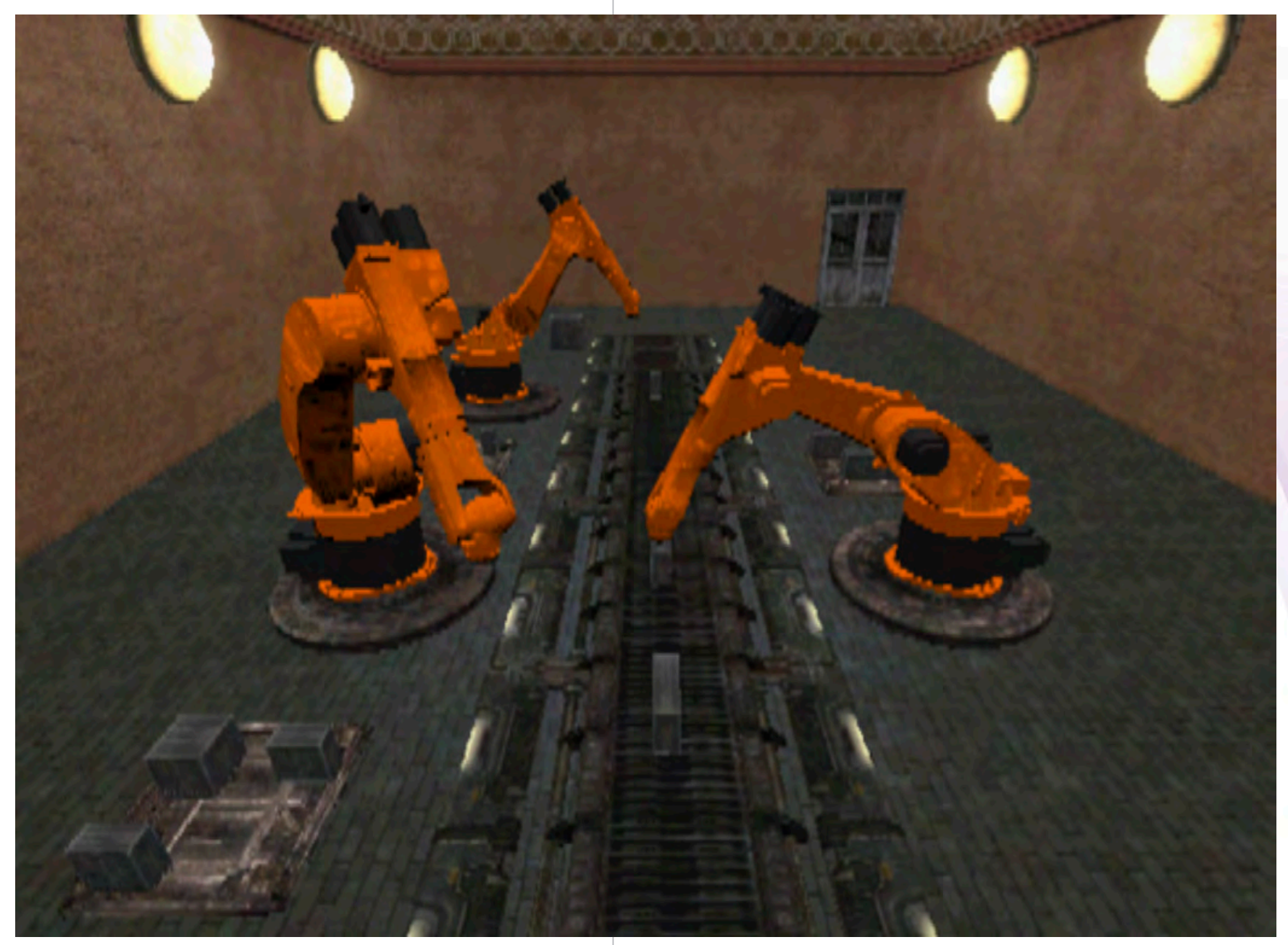

Three simulated robotic arms performing mixed palletizing utilizing the MOAST controller.

\section{Accomplishments}

- The Mobility Open Architecture Simulation and Tools (MOAST) and Unified System for Automation and Robot Simulation (USARSim) were recognized with the Government Open Source Conference "Engaging Citizens" award. This award was for promoting performance evaluation and benchmarking for robot systems and facilitating innovations in robotics.

- A full day workshop entitled "Robots, Games, and Research: Success stories in USARSim" was hosted at the 2009 IEEE/RSJ International Conference on Intelligent Systems and Robots. This workshop brought together government, industry, and academia for discussion and presentations on USARSim. Twelve papers were presented at this workshop along with discussion on the future of USARSim exposing many novices to technical issues within the international community.
- A subset of the USARSim interface standard (utilized by two different international competitions and numerous international researchers) was ported to a new, more capable game engine. This new game engine allows for more realistic physics simulation, a larger number of simulated bodies, and higher visual fidelity.

- Robot arms were added to the interface and several industrial quality arms were modeled. In addition to the arms, grippers were examined and modifications to the USARSim interface to support arms and grippers were explored. 
- The Virtual Manufacturing Automation Competition was successfully run at IEEE International Conference on Robotics and Automation. The competition featured autonomous unit loaders performing intra-factory transport of goods. This competition has been successful in focusing university researchers on manufacturing-related robotics, a subject that is otherwise largely neglected in U.S. universities, which favor military or service robotics.

\section{Planned Future Accomplishments}

- Complete the full port of the USARSim interface to the new game engine. This will provide a much better physics basis for the simulation and enable more complex simulations to be implemented.

- Extend the framework to include a study of "levels of validation". Different applications require different simulation fidelities and accuracies of validation. This framework will allow vendors and users of simulation to understand for what applications each simulation is appropriate.

- Extend the framework to include virtual humans known as avatars. These avatars will interact with both virtual and real systems and allow for safe testing of novel safety systems and robotic algorithms.

- Continue to work with industry and academia to develop performance standards for specific factory tasks such as intra-factory transport of goods and mixed palletizing.

\section{Customers and Collaborators}

Kuka Robotics, Georgia Institute of Technology, University of California-Merced, University of Freiburg, University of New Mexico, University of Zagreb, Croatia, Army Corps of Engineers 


\section{Advanced Control Systems and Positioning for Nanoscale Measurements and Standards}

\section{Challenge/Problem Addressed}

M etrology and fabrication of micro- and nanostructures are two key technologies for nano-manufacturing. A central challenge of micro- and nanomanufacturing is the development of methods to build complex three-dimensional (3-D) micro- and nano-scale structures and devices using techniques that allow them to interface with the macro scale world (scale up). The metrology of micro- and nano-structures, such as nanowires, nanoparticles, proteins, cells, etc., is a key enabling technology for the advancement of micro- and nano-technology and manufacturing, presenting many opportunities for novel micro- and nano-electromechanical and biomedical systems.

\section{Objectives}

- Develop world-class advanced control and positioning systems:

- for nanoscale measurements and standards to support emerging industries producing products or conducting research in micro- and nano-scale environments including nanowires, nanoparticles, proteins, cells, etc.

- for nanoscale manipulation and standards, to enable controlled positioning and assembly of micro- and nano-scale components for industries in a range of industries including electronics, medical, sensors, and custom materials

- Develop scale-up interfaces and standards between the micro- and nano-manufacturing tools and the macro scale world to enable processes to be visualized and controlled.

\section{Accomplishments}

- Transferred MEL research to industry through a Phase I Small Business Innovation Research (SBIR) project. EM Optomechanical received SBIR funding for "Micro-Positioner Replacement of Piezo Actuators in Long Working Distance Interference Microscopes"As a result of the interaction with MEL researchers EM
Optomechanical was able to design and build a prototype based on MEL research of the proposed high precision device.

- Invented, built prototypes of, and measured the performance of a MEMS nanopositioner embedded optical nano-displacement measurement sensor. The performance results indicate that sensor accuracy error ranges between 8 and 9 nanometers with the main source of error probably coming from the data acquisition PC card, algorithmic numerical errors and nanopositioner motion errors. Error sources will be investigated in FY2010. A patent disclosure application has been prepared. This sensor will be used for high precision nanopositioning and manipulation of nano-scale components for assembly and dynamic testing.

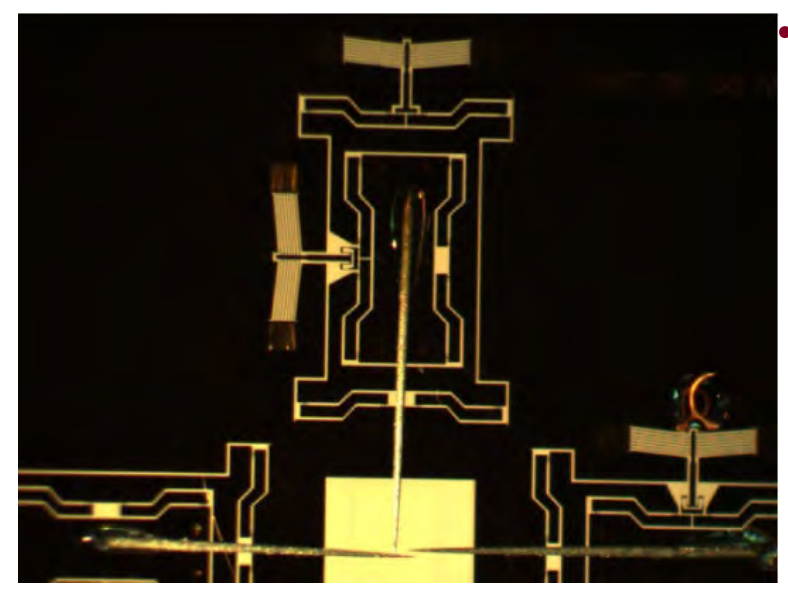

Array of planar MEMS nanopositioners fitted with nanoprobes for robotic manipulation. The external dimensions of each nanopositioner are approximately $3 \mathrm{~mm} \times 3 \mathrm{~mm}$.

- Invented, built prototypes of, and measured the performance of a dynamic nano rheology sensor in collaboration with NIST Material Science and Engineering Laboratory researchers. These are precision nano positioners for the measurement of the dynamic rheology properties of nano material solutions. The results of testing prototypes indicate that the sensors accurately measure dynamic rheology properties. A patent disclosure application has been submitted. The polymers and pharmaceutical industries will benefit from this work through being able to measure properties of very small quantities of chemical substances, including dynamic modeling of chemical structures. 
- Built and tested embedded piezoresistor nano force sensor prototypes to provide force feedback and stiffness measurements at the nano scale.

- John Wason, a PhD candidate from Rensselaer Polytechnic Institute, Troy, NY, has been using NISTfabricated artifacts to testing the performance of difficult automated micro assembly operations. This collaboration will result in automated assembly of a MEMS version of the MEL 6D nanopositioner/robotic manipulator, invented by MEL staff a few years ago.

\section{Collaborators and customers}

NASA, RPI, University of Maryland at College Park, University of Maryland at Baltimore County, George Washington University, NIST Material Science and Engineering Laboratory, NIST Electronics and Electrical Engineering Laboratory, NIST Information Technology Laboratory APNanotech, East Mountain Optomechanical

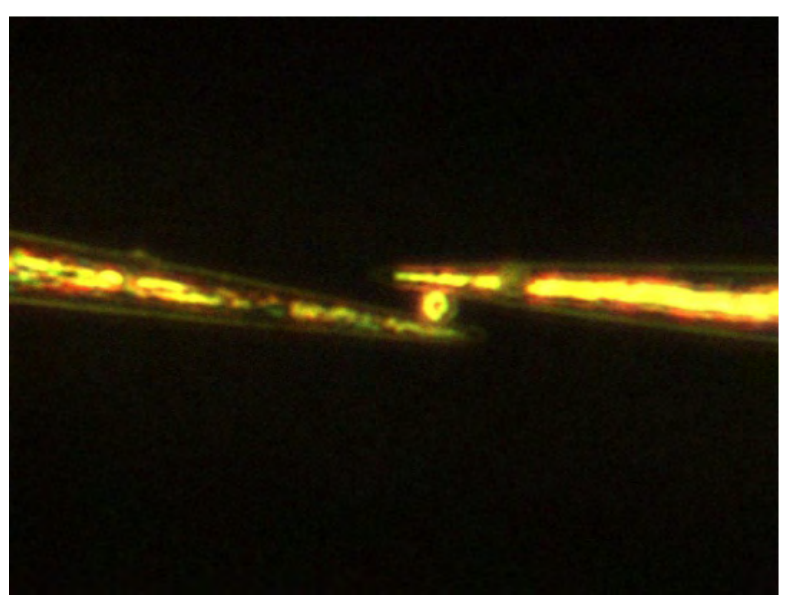

Coordinated manipulation of a 15 micro meter diameter particle by two nanopositioner nanoprobes.

\section{Publications}

J. C. Chiao, J. M. Goldman, D. A. Heck, P. Kazanzides, W. J. Peine, J. B Stiehl, D. Yen and N. G. Dagalakis, "Metrology and Standards Needs for Some Categories of Medical Devices," Journal of Research of the National Institute of Standards and Technology, Vol. 113, No. 2, pp. 121-129, March-April 2008.

G.F. Christopher, N. G. Dagalakis, S.D. Hudson, K.B. Migler, "MEMS Parallel-Plate Rheometer for Small Amplitude Oscillatory Shear Micro Rheology Measurements," Society of Rheology, $81^{\text {st }}$ Annual Meeting, Madison, WI, October 18-22, 2009.

J.D.Wason, W.T. Gressick, J.T.Wen, J.J. Gorman, N.G. Dagalakis, "Vision Guided Multi-probe Microassembly," Accepted by the IEEE/ASME Transactions on Mechatronics subject to modifications. 


\section{Nanorobotics and NEMS Measurement Science}

\section{Challenge/Problem Addressed}

$\Delta$ Ithough still in its infancy, the field of nanorobotics has the prospect of addressing a number of critical problems related to nanomanufacturing, biomedical research, and integrated nanotechnologies. As viewed in this project, nanorobots are mechanisms that can sense and manipulate the nanoscale environment using a combination of embedded sensors, actuators, control systems, and primitive logic. These robots may be tens of micrometers in size but will be composed of subcomponents with nanoscale dimensions. One of the most promising technologies for developing nanorobots is the field of nanoelectromechanical systems (NEMS), which is also in its early stages of development. As with macroscale mechanisms, measuring the motion and resulting reaction forces of nanorobots and NEMS is critical to understanding their mechanics. However, this is a significant challenge since many of the measurement methods typically used at the macroscale do not scale well and the measurement requirements are demanding (e.g., required force resolution can be on the order of piconewtons). Therefore, this project focuses on measurement science for nanorobotics and NEMS with an emphasis on nanoscale displacements and forces. Within this project there are three thrusts: 1) measurement methods based on macroscale instrumentation for off-chip mechanism characterization and calibration; 2) embedded sensing within nanorobots and NEMS for real-time measurement and control; and 3) the application of micro/nanoelectromechanical systems (MEMS/ NEMS) to the design of advanced nanometrology and nanomanufacturing systems.

\section{Objectives}

- Develop near-field and far-field optical methods for measuring the in-plane and out-of-plane motion of nanorobots and NEMS with sub-nanometer resolution and Radio frequency (RF) bandwidth. These methods will be used to evaluate the electromechanical dynamics of nanoscale mechanisms and provide traceable calibration for embedded nanoscale displacement sensors.

- Develop NEMS sensors for measuring nanoscale displacement and force for embedded sensing in nanorobots, and process characterization and control in nanomanufacturing. The priority of this research is on increasing both the resolution and measurement rate compared to existing MEMS technologies.

- Utilize MEMS/NEMS to develop next-generation scanning probe instruments for nanometrology and nanomanufacturing. In particular, MEMS/NEMS are used to dramatically increase the scan rates for nanometrology methods such as atomic force microscopy and scanning tunneling microscopy as well as increase the number of active scanning probes. 


\section{Technical accomplishments}

- Successfully designed, fabricated, and characterized a prototype MEMS scanning tunneling microscopy (STM) scanner with an embedded displacement sensor. The achievable scan rate is higher than 80,000 $\mathrm{nm} / \mathrm{s}$, which is at least ten times faster than conventional STM.

- Successfully completed Phase I of the DARPA TipBased Nanofabrication program ahead of schedule. This will enable manufacture of nanostructures, specifically nanowires, nanotubes, and quantum dots, with nanometer-scale control over the size, orientation, and position of each nanostructure. Phase II of the program, already awarded, will require the development of a 5 X 1 MEMS STM array that can image silicon surfaces with atomic precision.

\section{Planned Future Accomplishments}

- Demonstrate the measurement of out-of-plane NEMS motion with sub-nanometer resolution and $1 \mathrm{Mhz}$ bandwidth using a diffraction-limited homodyne interferometer that will be part of the nanoscale motion microscope (NMM). (Complete by 3rd Quarter 2010)

- Demonstrate structural modal analysis of a NEMS using a custom near-field scanning optical microscope (NSOM) that will achieve $50 \mathrm{~nm}$ spatial resolution or better.

- Fabricate and characterize a piezoelectric resonant NEMS force sensor that is designed to measure force gradients (as present in atomic force microscopy) by tracking frequency shifts in its resonant frequency in the $\mathrm{MHz}$ range.

- Demonstrate imaging of (100) silicon with atomic resolution in UHV using a closed-loop controlled 3-axis MEMS-based STM scanner.

\section{Customers and Collaborators}

DARPA, Zyvex Labs, Integrated Circuit Scanning Probe Instruments (ICSPI), University of Waterloo, University of Michigan 
changes. For several years, these executives focused on product innovation as a driver of competitive advantage, recognizing that innovation is a means to achieve all those other goals. More recently their understanding of what enables innovation has changed, leading to a new focus not just on innovating products, but also on innovating business processes and business models that influence the creation of innovative products. Product development takes place in a complex network of partners as shown in Figure 1. This, in turn, has elevated the status of product lifecycle management (PLM) from being merely an engineering concern to an enterprisewide business concern, with emphasis on information integration and collaboration with partners. ${ }^{1}$ Achieving, assuring, and maintaining global interoperability is too large a task for all but the biggest corporations. We at NIST are increasingly asked by our nation's companies to help address the interoperability problem at the global level.
Among these are international modeling language standards. Their impact is that they create common languages for systems' communication, and thus create "speech communities" that do not depend on a particular choice of vendor. They contribute to training analysts and engineers, providing vocabularies and a constant way to represent the manufacturing enterprise concepts and provide a means of communication, one community to the other. This enables more people to communicate with each other throughout the manufacturing process. It also contributes toward bridging engineering and business disciplines throughout the PLM. MSID continues to bridge ISO 10303 (Standard for the Exchange of Product model data - STEP) application protocols to these modeling language standards. Another notable standardization achievement is the Simulation Interoperability Standard's Organization Core Manufacturing Simulation Data specification standardization. MSID continues to enhance on-line testing ser-

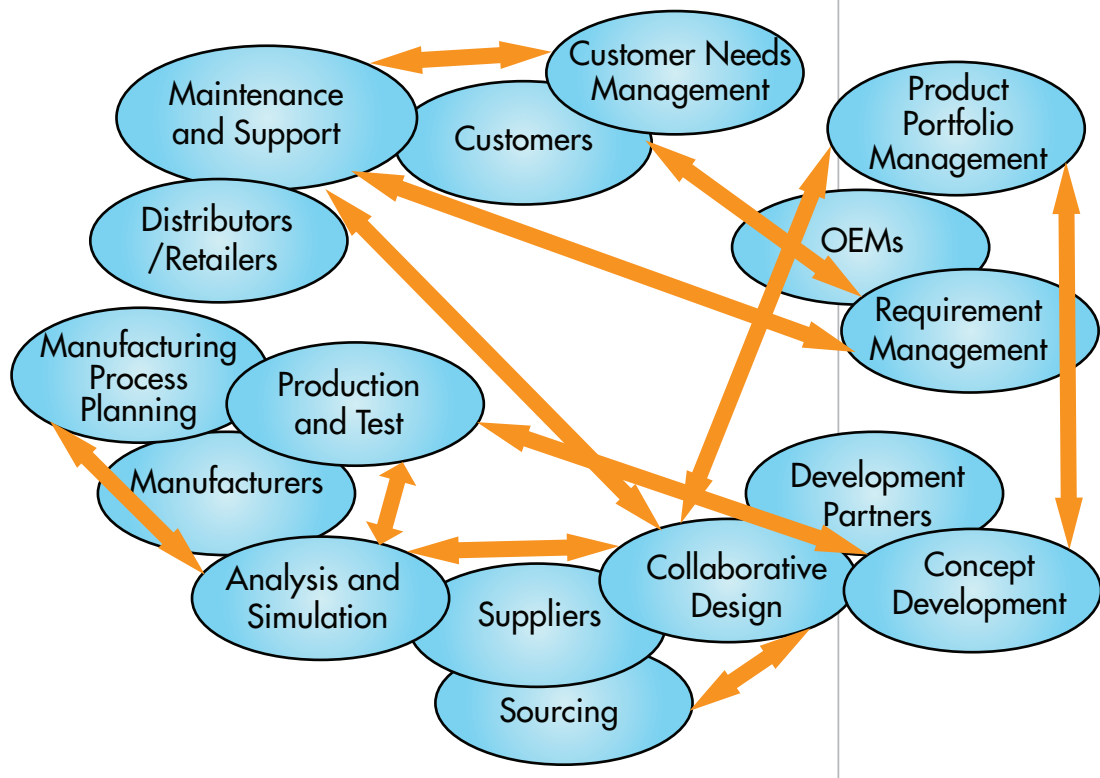

Figure 1: Complex network of PLM partners

Working with industry, MSID is helping to devise sustainable solutions for this multifaceted and global challenge in supply chain requirements and the business aspects of PLM. We have led the way in developing several major international standards for exchanging product information and for managing the torrents of data that businesses now must organize and process.

1 Srinivasan V. A comprehensive business approach to product realization using service oriented architecture. In: Tomovic M, Wang S, editors. Product realization: A comprehensive approach. Springer; 2009. vices that enable design-engineering, supply chain, and e-business software vendors to validate the conformance of their products to emerging or existing standards, and provides tools to the standardization community to ensure rigorous quality for the standards themselves.

MSID has selected to focus and address a set of key industrial "pain points" for maximum impact. The selected set includes: (1) improving the information flow across the supply chain, (2) gathering and disseminating best practices in sustainable manufacturing, and (3) identifying meaningful sustainability metrics that lead to improvements. In choosing our work, we have been influenced by several major industrial drivers that reflect significant trends relating to information exchange in the manufacturing industry:

- Globalization

- Off-shoring of suppliers

- Increases in foreign markets for U.S. manufacturers

- Concentration on core competencies within manufacturing companies 
- Intermixing of previously separate industrial "stovepipes" between sectors

- Sustainable manufacturing

- Increased attention to the entire product lifecycle, including disposal or recycling

- Growing regulatory pressure on hazardous waste production, greenhouse gas emissions, and energy consumption

\section{The implications of these drivers include:}

- Globalization creates increased dependence on electronic information exchange.

- Globalization requires integration between formerly separate industry sectors, each with entrenched practices and terminologies.

- Sustainable manufacturing necessitates richer information structures to capture more than simple product geometry.

- Increased emphasis on sustainability has focused attention on the supply chain --- one is only as green as its supply chain. It has now become a new practice to refer to a "closed-loop" supply chain, which includes both the forward and the reverse supply chains.

- Complex systems increase the difficulty of achieving interoperability.

\section{In response to these drivers, MSID has two programs:}

- Sustainable and Lifecycle Information-based Manufacturing

- Supply Chain Integration

- 34 guest researchers

- $\$ 9,909.9$ K STRS

\section{Staffing Challenges:}

- Attracting postdoctoral researchers continues to remain difficult, even though the economic downturn has generated a little more interest. NIST's entry-level salary structure for postdoctoral researchers, relative to that of engineers and computer scientists in the private sector, continues to remain a problem complicated further still by the requirement that postdoctoral research candidates must be U.S. citizens.

- Our permanent staff has a top-heavy demographic - senior staff have topped-out salaries and we cannot replenish junior employee ranks, as our budget remains relatively static. This also influences the appeal to supervise formally, as there is no financial compensation for managers who have topped-out salaries.

\section{Facilities and} Equipment

- 26 permanent NIST staff

- 1 part-time permanent, technical

- 1 NRC post-doctoral researcher

FY2010 Estimated Funding

- \$602.454 K Other Agency

dvanced Manufacturing Systems And Networking Testbed (AMSANT) and Simulation Laboratories: these are multi-purpose computer laboratories that support the Manufacturing Engineering Laboratory staff and a host of other project-specific applications for other NIST operating units and other agency sponsors. Both laboratories provide a multi-media environment of computers, audio, video, wireless, and networked distribution systems. The Simulation Laboratory complements the services provided by the AM-

SANT, offering a wide variety of simulation tools, as well as a 3D laser scanner that support modeling in manufacturing and other domains. The Simulation Laboratory allows us to build manufacturing simulations to support testing objectives and work with industrial partners using the same tools they use in real-time. Both laboratories foster a collaborative physical and virtual environment for worldwide communication and demonstration.

\section{Facilities and Equipment Challenges:}

$\mathrm{T}$

he continuing drop in the price of computing systems allows us to provide excellent computing support for our work in a cost-effective manner without technical capability degradation. 


\section{Sustainable and Lifecycle Information-based Manufacturing}

To prepare for a future where manufacturing has a zero net impact on the environment, the United States industry will require key resources and methods that will enable it to measure sustainability along several dimensions allowing accurate assessment of status and progress. These resources and methods require a science-based identification of dimensions, associated measurements and classification and characterization of information relevant to sustainable products, processes, and services. Such traceable information is critical to product designers and manufacturing engineers so that they can incorporate sustainability in their efforts and comply with international regulations. To create this information infrastructure the program will: analyze standards requirements and best practices for sustainable manufacturing; create lifecycle information models for interoperability among systems and tools that support sustainable manufacturing; and validate and test information models for sustainable design and manufacturing.

\section{Staff}

8.75 NIST Staff

9 Guest Researcher FTEs

17.75 Total FTEs

\section{Challenge}

$s$ green thinking enters the economic mainstream, U.S. industry is considering how to employ cradleto-cradle strategies to lessen the environmental impact of manufacturing. Monitoring the lifecycle of a fully recyclable, sustainable product -- to include modeling the evolution of a product across its lifecycle on a computer, including its design, manufacture, use and eventual disposal -- requires clear science-based methods and traceable information to define, assess and measure various indicators of sustainability. Currently manufacturers are confronted with too many choices involving ethical decisions and often vague concepts. How to interpret sustainability according to products, industry sector, regional and global factors? Must the carbon footprint of every single object used be taken into account? Is it better to send disposable products to a landfill or to clean reusable products with potential waste of energy, water and perhaps chemicals? Having government and public agreement on these concepts is only the first step -- manufacturers using computer models to find the best practices to accommodate a triple bottom line of economics, environmental stewardship, and social relevance need to know that the software they rely on embodies a quantitative, precise understanding of this new green territory. The manufacturing floor, with its dependence on enterprise wide and interoperable software across the supply chain, simply cannot afford to use ill-defined concepts. Precise information exchange between design, engineering, manufacturing and endof-life steps is the key for producing sustainable products. Failures in information exchange at the interfaces between design, engineering, manufacturing and other functions can be viewed as the Achilles heel of good product development. Further, for green manufacturing systems to achieve their goals, rigorous, clearly defined measurements for sustainable indicators are essential.

\section{Technical Strategy}

- he Sustainable and Lifecycle Information-based

Manufacturing program has three primary objectives. First is providing key standards requirements and best practices for sustainable manufacturing. This includes analyzing and defining the relevant standards from carbon footprint determination to energy resource management to hazardous material management. The program is also developing a scheme for computing the carbon footprint of a manufactured product -- a critical mathematical "score" that can help resolve concerns such as the issue of reusable versus disposable products. NIST is looking to ensure that new standards mesh 
with current practices, so researchers are working to harmonize their green information standards with existing software standards for manufacturing product data, such as ISO 10303, the Standard for the Exchange of Product model data (STEP).

Second, the program is providing a framework for environmental manufacturing models by determining the key attributes and developing information models necessary for sustainable manufacturing. For example, a model that truly takes into account all environmental impacts would have to include, among other things, the energy costs of making the equipment that is used to manufacture the product and the cost of recycling the product at the end of its lifetime. Additionally, to understand and calculate the historical environmental impacts of products, processes, and services the program is developing strategies to support storage and access for long-term archiving of engineering lifecycle information.

Third, researchers are developing protocols for testing and simulating the application of green standards. Current manufacturing digital simulations would need to be extended to address such additional issues such as energy consumption, pollution, unnecessary transportation costs, and inventory stockpiling. NIST will also develop a test bed that will allow comparison of computer simulation predictions with physical prototypes.

\section{Results and Impacts}

O rganized two workshops, held in Bangalore, India and at NIST respectively, which brought together experts and various stakeholders to identify and discuss measurements and standards for sustainable manufacturing, and to understand the global issues. The workshops identified major challenges facing the industry and key recommendations to address these challenges and were documented in the workshop reports.

Developed formal process semantics included in a major revision of the Business Process Model and Notation (BPMN), Systems Modeling Language, and Unified Modeling Language, as standardized by the Object Management Group (OMG). This significantly improved the interoperability and precision of standards widely used in manufacturing. Impact of this work is underscored by the following statement from IBM's Vice President of Standards: "The emerging OMG BPMN 2.0 standard will facilitate productivity improvements and ongoing business process innovation across many of the industrial and manufacturing sectors IBM serves."

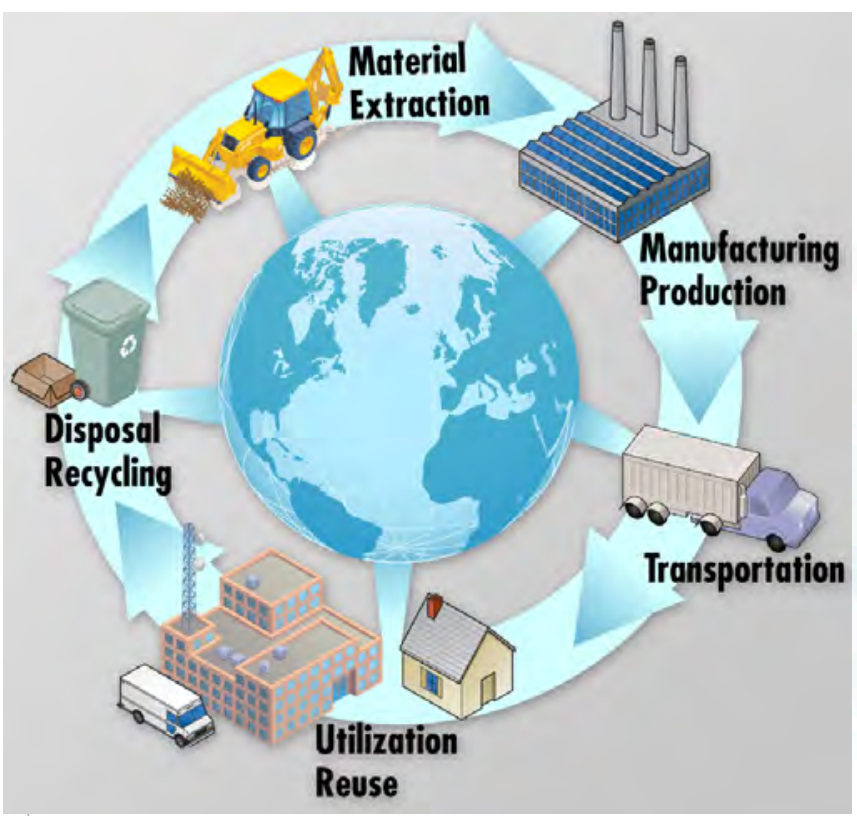

Created and implemented mappings from ISO 10303 11 ("STEP" EXPRESS) to the Unified Modeling Language (UML), UML Profile of EXPRESS, and EXPRESS to the Web Ontology Language (OWL) for data exchange and reasoning. These mappings and tools make existing STEP data exchange models useful to the current generation of software developers. For example, PDES Inc. has plans to put a link to one of the mapping tools - OntoSTEP on their consortium web site, which will result in wide dissemination of OntoSTEP.

\section{Publications}

"An Analysis of Description Logic Augmented with Domain Rules for the Development of Product Models," X. Fiorentini, S. Rachuri, H. Suh, J. Lee, J., and R. D. Sriram, Journal of Computing and Information Science in Engineering, Vol. 10, March 2010.

"Carbon Weight Analysis for Machining Operation and Allocation for Redesign," G. Ameta, M. Mani, S. Rachuri, S. C. Feng, R. D. Sriram, K. W. Lyons, International Journal of Sustainable Engineering, Volume 2, Issue 4, 2009, pages $241-251$.

"Sustaining Engineering Informatics: Toward Methods and Metrics for Digital Curation," J. Lubell, S. Rachuri, M. Mani, E. Subrahmanian, The International Journal of Digital Curation Issue 2, Volume 3, 2008.

\section{Contact Information:}

Ram D. Sriram, Program Manager

(301) 975-3507

ram.sriram@nist.gov 


\section{Awards and Recognition}

\section{Board Membership}

\begin{tabular}{|l|l|}
\hline Staff & Board Membership \\
\hline Ram D. Sriram & - Member Editorial Board, "Research for Engineering Design," Springer Verlag \\
& - Advisory Board, Several international conferences \\
& - Scientific Officer, National Center for Biomedical Ontologies \\
& - Advisory Board, Journal of Concurrent Engineering: Research and Applications \\
\hline Shaw Feng & - Member, Editorial board of the International Journal of Sustainable Manufacturing. \\
\hline Guodong Shao & • Executive Board member, Winter Simulation Conference \\
\hline
\end{tabular}

\section{Leadership}

\begin{tabular}{|c|c|}
\hline Staff & Leadership \\
\hline Ram D. Sriram & $\begin{array}{l}\text { - Member, Executive Committee, Computers in Engineering Division and organized } \\
\text { several panels in ASME IDETC/CIE 2009, August. } \\
\text { - Panel organizer, Shared Ontologies for Engineering Design, ICORD'2009, January, } \\
\text { Bangalore, India } \\
\text { - Panel organizer, Healthcare Automation and Sustainable Manufacturing, IEEE CASE } \\
\text { 2009, August, Bangalore, India }\end{array}$ \\
\hline Rachuri Sudarsan & $\begin{array}{l}\text { - U.S. Regional Editor, International Journal of Product Development (IJPD) } \\
\text { - Associate Editor, International Journal of Product Lifecycle Management (IJPLM) } \\
\text { - Special Issue Co-Editor, IJPLM - Developing Sustainable Products, Processes And } \\
\text { Services, Vol. 4, No. 1-3, } 2009 \\
\text { - Guest editor, Special issue International Journal of Product Lifecycle Management } \\
\text { (IJPLM), "Exploring the Role of Knowledge Management Technologies in PLM". } \\
\text { - Co-Chair, Indo-US Workshop on Designing Sustainable Products, Services and } \\
\text { Manufacturing Systems, August, 2009, Indian Institute of Science (IISc), Banga- } \\
\text { lore, India. } \\
\text { - Workshop Chair, NIST Workshop on Sustainable Manufacturing: Metrics, Stan- } \\
\text { dards, and Infrastructure, October 2009, NIST, Gaithersburg, MD, USA } \\
\text { - Program Committee Member, International Conference on Software Knowledge } \\
\text { Information Management and Applications (SKIMA) 2006, 2008, 2009. } \\
\text { - Program Committee Member, International Product Lifecycle Management Confer- } \\
\text { ence, 2003-present. } \\
\text { - Special Session and Panel Organizing Chair, 2008 ASME International Design } \\
\text { Engineering Technical Conferences (IDETC) and Computers and Information in } \\
\text { Engineering Conference (CIE). }\end{array}$ \\
\hline
\end{tabular}




\begin{tabular}{|c|c|}
\hline Staff & Leadership \\
\hline Rachuri Sudarsan & $\begin{array}{l}\text { - Organizer, Special Session "The role of ICT in Sustainable Manufacturing Systems", } \\
\text { Fifth Annual IEEE Conference on Automation Science and Engineering (IEEE CASE } \\
\text { 2009), IEEE Robotics and Automation Society, Bangalore, India. } \\
\text { - Organizer for sessions and panels, AMSE DETC Conference, 2009, San Diego, CA. } \\
\text { - Associate Member (invited) of CIRP (College International pour la Recherche en } \\
\text { Productique-International Academy for Production Engineering) }\end{array}$ \\
\hline Conrad Bock & $\begin{array}{l}\text { - Panel organizer, Open Standards for Model-based Enterprise and Engineering } \\
\text { Integration, American Society of Mechanical Engineers, San Diego, California, } \\
\text { September, } 2009 . \\
\text { - Lead and editor for Formal Semantics Workgroup in Semantics Of A Foundational } \\
\text { Subset Of Executable UML Models Finalization Task Force at the Object Manage- } \\
\text { ment Group. } \\
\text { - Lead and editor for the Activities Workgroup in the Systems Modeling Language } \\
\text { Revision Task Force at the Object Management Group. }\end{array}$ \\
\hline Prabir Sarkar & $\begin{array}{l}\text { - Special Issue Co-Editor, IJPLM, Developing Sustainable Products, Processes And } \\
\text { Services, Vol. 4, No. 1-3, } 2009 \\
\text { - Chief Editor, International Journal of Product Design, Serials Publications. } \\
\text { - Organizing member, Indo-US Workshop on Designing Sustainable Products, } \\
\text { Services and Manufacturing Systems, August 2009, Indian Institute of Science } \\
\text { (IISc), Bangalore, India. }\end{array}$ \\
\hline Swee Leong & $\begin{array}{l}\text { - Chair, Simulation Interoperability Standards Organization (SISO) Core } \\
\text { Manufacturing Simulation Data (CMSD) Product Development Group standards } \\
\text { development effort } \\
\text { - Lead: Simulation Standards Consortium }\end{array}$ \\
\hline Mahesh Mani & $\begin{array}{l}\text { - Symposium Organizer: Modeling Tools and Metrics for Sustainable Manufactur- } \\
\text { ing; ASME-IDETC/CIE 2010, August 2010, Montreal, Canada } \\
\text { - Symposium Co-organizer and Session Chair: Modeling Tools and Metrics for Sus- } \\
\text { tainable Manufacturing; ASME-IDETC/CIE 2009, August 2009, San Diego, Califor- } \\
\text { nia. } \\
\text { - Organizer Special Session: Performance Metrics for Sustainable Manufacturing; } \\
\text { Performance Metrics for Intelligent Systems Workshop, National Institute of Stan- } \\
\text { dards and Technology, Gaithersburg, September } 2009 \\
\text { - Coordinator: Panel on Performance Metrics for Sustainable Manufacturing, Perfor- } \\
\text { mance Metrics for Intelligent Systems Workshop, National Institute of Standards } \\
\text { and Technology, Gaithersburg, September } 2009 \\
\text { - }\end{array}$ \\
\hline
\end{tabular}




\begin{tabular}{|c|c|}
\hline Staff & Leadership \\
\hline Guodong Shao & $\begin{array}{l}\text { - Conference committee member, Industrial Simulation Conference } 2008 . \\
\text { - Track chair, Virtual Reality and Graphical Simulation for Industrial Simulation Con- } \\
\text { ference } 2008 . \\
\text { - Session chair, Winter Simulation Conference } 2008 . \\
\text { - Advisory chair, SIMUL } 2009 \text {-The International Conference on Advances in System } \\
\text { Simulation } \\
\text { - Conference committee member, SIMUL } 2009 \text {-The International Conference on } \\
\text { Advances in System Simulation } \\
\text { - Conference committee member, Industrial Simulation Conference } 2009 . \\
\text { - Track chair, Virtual Reality and Graphical Simulation for Industrial Simulation Con- } \\
\text { ference } 2009 . \\
\text { - Session chair, Industrial Simulation Conference } 2009 . \\
\text { - Conference committee member, North American Simulation Technology Confer- } \\
\text { ence (NASTEC) } 2009 . \\
\text { - Advisory chair, SIMUL } 2010 \text {-The International Conference on Advances in System } \\
\text { Simulation } \\
\text { - Conference committee member, SIMUL } 2010 \text {-The International Conference on } \\
\text { Advances in System Simulation } \\
\text { - Conference committee member, North American Simulation Technology Confer- } \\
\text { ence (NASTEC) } 2010 .\end{array}$ \\
\hline Kevin Lyons & $\begin{array}{l}\text { - Co-editor, Special Nanotechnology issue of the ASME Journal of Manufacturing } \\
\text { Science and Engineering, Summer } 2010 \\
\text { - Session chair, NanoMaterials for Defense } 2008 \text { Conference, half-day plenary ses- } \\
\text { sion entitled Nanoscale Production Challenges. } \\
\text { - Co-organizer and session co-chair, NSF workshop, "Grand Challenges for Bio- } \\
\text { Nanomanufacturing for Year 2020, instrumentation, metrology, and standards for } \\
\text { bio-nanomanufacturing areas. } \\
\text { - Moderator and presenter, Nanomanufacturing Panel at the ASME Micro- and Nano- } \\
\text { systems (MNS) Conference 2008. } \\
\text { - Symposium co-organizer, Modeling Tools and Metrics for Sustainable Manufactur- } \\
\text { ing; } 2009 \text { ASME International Design Engineering Technical Conferences (IDETC)/ } \\
\text { Computers in Engineering (CIE) for } 2009 \text { and } 2010\end{array}$ \\
\hline $\begin{array}{l}\text { Allison Barnard } \\
\text { Feeney }\end{array}$ & $\begin{array}{l}\text { - Project Editor, ISO 10303-233 Application Protocol: Systems Engineering within } \\
\text { ISO TC 184-SC } 4 \text { Industrial Data. }\end{array}$ \\
\hline
\end{tabular}




\begin{tabular}{|c|c|}
\hline Staff & Excellence Recognized \\
\hline Ram D. Sriram & $\begin{array}{l}\text { - Elected as a Fellow of the American Association for the Advancement of Science, } \\
2010 . \\
\text { - Keynote Speaker, TEKES' launch of a three year } 100 \text { million euros Digital Product } \\
\text { and Process initiative (5/7-5/9) in Finland. } \\
\text { - Keynote Speaker, Tools and Methods of Competitive Engineering 2008, April } 2008 \\
\text { (Topic: Information Modeling Framework and Ontology Development for PLM) }\end{array}$ \\
\hline Rachuri Sudarsan & $\begin{array}{l}\text { - ASME Certificate of Appreciation, Contribution to ASME Y14.5.1 standard and } \\
\text { ASME Y14.5.1 working group participation } \\
\text { - Received testimonies from industry leaders from Xerox, Ford, and URS Corporation } \\
\text { regarding work on sustainability related research and leadership } \\
\text { - Keynote talk, Model based Engineering and Standards for Sustainable Manufactur- } \\
\text { ing System, CIRP meeting, "The Role of Interoperability Standards in Enabling Sus- } \\
\text { tainable Enterprise", Fifth International Conference on Signal Image Technology } \\
\text { and Internet Based Systems (SITIS 2009), November 2009, Marrakech, Morocco }\end{array}$ \\
\hline Conrad Bock & $\begin{array}{l}\text { - Received testimonial letters from John Deere, SAP, International Business } \\
\text { Machines, and Lockheed for work on formalization and standardization of process } \\
\text { and products models. }\end{array}$ \\
\hline $\begin{array}{l}\text { Allison Barnard } \\
\text { Feeney }\end{array}$ & $\begin{array}{l}\text { - Member, INCOSE Model Driven System Design Working Group, received INCOSE } \\
\text { Working Group Award for bringing the STEP Systems Engineering standard (AP233) } \\
\text { to completion. } \\
\text { - Received testimonial letters from John Deere and Lockheed Martin for work align- } \\
\text { ing systems engineering standards across INCOSE, OMG and ISO TC 184-SC } 4 .\end{array}$ \\
\hline Josh Lubell & $\begin{array}{l}\text { - Testimonials from the Balisage Markup Conference chairs and also from the inven- } \\
\text { tor of the Schematron XML schema language for the paper "Documenting and } \\
\text { Implementing Guidelines with Schematron." }\end{array}$ \\
\hline Kevin Lyons & $\begin{array}{l}\text { - Invited member, NSF Nanomanufacturing Center panel to review the NSF Center } \\
\text { for Nanoscale Chemical-Electrical-Mechanical Manufacturing Systems (Nano- } \\
\text { CEMMS) located at the University of Illinois. } \\
\text { - Invited participant, MEL representative, Manufacturing Trends } 2020 \text { strategic plan- } \\
\text { ning workshop sponsored by NIST and organized by SRI Consulting: Business } \\
\text { Innovation (SRIC-BI) to identify potential research directions for NIST in the area of } \\
\text { manufacturing. }\end{array}$ \\
\hline Shaw Feng & $\begin{array}{l}\text { - Invited to join the ASME Sustainable Manufacturing Research Committee to } \\
\text { lead and coordinate efforts in developing sustainability metrics and } \\
\text { measurement methods. }\end{array}$ \\
\hline
\end{tabular}


Sustainable and Lifecycle

Information-based

Manufacturing Projects

\section{Sustainability Standards Landscape}

\section{Challenge/Problem Addressed}

T here is a variety of voluntary and regulatory standards related to sustainability. Selecting applicable standards across the entire lifecycle is a critical and challenging task for manufacturers. Using these standards one can develop best practices and recommended practices to reduce environmental impact of products, processes, and services. To develop these practices it is critical to understand these standards from the information and modeling point of view that will take into account requirements of various stakeholders. Further, to assess the impact of recently proposed international or national standards, and to compare the effect of various applicable standards on products, processes, and services it is critical to understand the impacts of changes proposed in these sustainability standards.

\section{Objective(s)}

- Develop a typology of mandatory and voluntary standards related to sustainability.

- Analyze each standard from the information and modeling point of view that will take into account requirements of various stakeholders such as customer, researcher, government, industry and others.

- Develop a web-portal to help various stakeholders to study and compare standards in a formal way using ontologies.

- Analyze standards using the Zachman framework for gaps and overlap analysis, and help industries to understand issues related to standard implementation.

- Develop a repository of indicators and measures for sustainability of products, processes, and services using triple bottom line.

\section{Accomplishments}

- Analyzed a large set of standards related to sustainability such as ISO 14000, REACH (Registration, Evaluation, Authorization and Restriction of Chemicals), RoHS (Restriction of Hazardous Substances) and related standards for the web-portal and developed a web-portal that consolidates information on sustainability related standards. These standards were analyzed using a Zachman framework. Plans are underway to include this web-portal as part of the DOC/ITA's (U.S. Department of Commerce/International Trade Administration) Sustainable Business Clearinghouse, which will enable various stakeholders to implement sustainability related standards in a meaningful manner.

- Developed a method for assessing sustainability. The measure is based on energy and material flow and it is designed in such a way that a designer can do comparative analysis among the design alternatives, incomplete and evolving information. Based on this sustainability impact measure, the industry can define a "baseline impact of product", which does not exist now.

\section{Planned Future Accomplishments}

- Analyze standards using Zachman framework for gaps and overlap analysis, and help industries to understand issues related to standard implementation.

- Evaluate the above newly developed method for assessing sustainability of products in industries.

\section{Customers and Collaborators}

Department of Commerce/International Trade Administration (DOC/ITA); Environmental Protection Agency; American National Standards Institute; Aerospace Industries Association; American Society of Testing and Materials E60; Washington State University; Xerox Corporation; The Boeing Company. 


\section{Sustainability Indicator Repository}

\section{Challenge/Problem Addressed}

\begin{abstract}
key challenge for manufacturing companies is to overcome the barrier in measuring sustainability performance throughout a product's life cycle. In an attempt to address the challenge, many sustainability indicators have been developed by different international, national, and private organizations. There are overlaps and inconsistency among the developed indicator sets. An emerging industry need is on an open, neutral, and inclusive indicator set.
\end{abstract}

\section{Objective(s)}

- Develop an indicator repository that provides harmonization to existing indicators. Sustainability indicators in the repository include those that are harmonized with existing sets, others that are derived industry practices, and the others that are identified as additional needed indicators to meet industry needs.

- Provide definitions of fundamental terms frequently used in sustainability measurement, indicator characteristics of the sustainability indicators, the format/template of the statement of the measurement purpose, and the need of sustainable manufacturing metrics.

- Develop suggested Indicator hierarchies, which are developed using a structure of categories and the relationship with the product life cycle. Also, develop example applications of the repository to facilitate understanding. Include the developed indicator hierarchies and examples in the indicator repository.

\section{Accomplishments}

- Developed an initial measurement infrastructure for sustainable manufacturing and published an overview of the infrastructure in the 7th Global Conference on Sustainable Manufacturing, December 2009. This will enable the U.S. manufacturing industry to measure sustainability in their products and processes and will help them to compete in the international markets.

\section{Planned Future Accomplishments}

- Publish an overview of sustainable manufacturing metrics development at NIST in the $17^{\text {th }}$ CIRP Life Cycle Engineering Conference in May 2010.

- Develop an indicator repository that provides harmonization to existing indicators, defining fundamental terms frequently used in sustainability measurement.

- Create a web site of the sustainability indicator repository for manufacturing companies to retrieve necessary indicators to measure their sustainability performance in products and processes.

\section{Customers and Collaborators}

Organization of Economic Cooperation and Development; University of Kentucky, Texas Tech University; GE Aviation, Lexmark, Toyota, Proctor and Gamble 


\section{STEP Evolution and}

\section{Transition}

\section{Challenge:}

ne key factor in the success of many best-of-breed enterprises is efficient information management. Increasingly, enterprises are employing Model Driven Architectures, semantics, ontologies and Semantic Web technologies to successfully enable efficient and integrated information management.

The STEP and related ISO TC 184 SC4 Industrial Data standards developed over the past two decades provide a rich source of consensus-based domain-specific information models. Production implementations of STEP have resulted in real cost savings of $\$ 150 \mathrm{M}$ per year to US industry. However, STEP lacks a consistent IT architecture based on widely used technologies. The STEP focus on data means that its standards do not address other viewpoints that have become key to business process reengineering and systems integration over the past several years.

Enabling interoperability between STEP and the technologies standardized in OMG and $\mathrm{W} 3 \mathrm{C}$ enables the proven information models of STEP to be part of an enterprise integration solution based on inter-related data exchange, ontology and service specifications.

Accordingly, tools and methods are needed to allow reuse of existing STEP standards within an evolving standards infrastructure, and to help manufacturers ensure that established STEP implementations can be retooled to work with more widespread implementation technologies.

\section{Objectives}

- Enable engineering enterprise integration based on inter-related data exchange, ontology and service specifications

- Greatly reduce barriers to the use of SC4 standards in industry by enabling the successful integration of STEP with OMG and W3C standards

- Demonstrate a process for migration of STEP data models to more widely implemented modeling and implementation technologies.

\section{Accomplishments}

- Created and implemented mappings from STEP EXPRESS to Unified Modeling Language (UML), UML Profile of EXPRESS, and EXPRESS to Web Ontology Language (OWL) for data exchange and reasoning. The mappings and tools developed under this project make existing STEP data exchange models useful to the current generation of software developers.

- Led the publication of STEP standard for exchanging Systems Engineering information (AP233), which will result in significant reduction in time and costs of developing complex systems.

- Created, implemented, and demonstrated a mapping between OMG's Systems Modeling Language (SysML) and STEP AP233 (Application Protocol 233, the STEP Systems Engineering project), which will facilitate interoperability of systems engineering (SE) tools with other SE tools and computer-aided engineering software.

- Initiated project to propose Future SC4 (ISO 10303, Technical Committee 184, Sub-committee 4 on Industrial Data) Architecture within the ISO subcommittee responsible for STEP development. The proposed Future SC4 Architecture supports direct use of OMG and $\mathrm{W} 3 \mathrm{C}$ standards and enables enterprise integration based on inter-related data exchange, ontology and service specifications.

\section{Planned Future Accomplishments}

- Publish proposed Future SC4 Architecture for SC4 ballot.

- Make freely available the mapping specifications and tools that enable integration of STEP and OMG and W3C standards and technologies.

- Develop methods and guidelines for implementing the Future SC4 Architecture.

- Identify and work with a pilot project to demonstrate that the Future SC4 Architecture enables enterprise integration based on inter-related data exchange, ontology and service specifications.

\section{Customers and Collaborators}

PDES, Inc.; ISO TC184/SC 4 Industrial Data; Object Management Group (OMG); World Wide Web Consortium (W3C); Eurostep, Inc.; John Deere; Lockheed Martin 


\section{Information Models for Sustainable Manufacturing}

\section{Challenge/Problem Addressed}

anufacturing has become increasingly com-

$\checkmark$ plicated due to many new factors that must be considered, such as sustainability, and a wider range of collaborators involved. Engineers interact across a wide range of specialties, especially to account for an increasing number of process alternatives in the reverse supply chain, such as disassembly and recycling. Consideration of multiple lifecycle stages and more collaboration result in better and more sustainable products, but also place a significant burden on engineers to examine a larger set of alternative designs at varying levels of detail. Collaboration is often hampered by lack of uniform interpretation of modeling languages and terminologies, leading to rework when discrepancies are discovered.

A major aspect of sustainability is the reverse supply chain, which prepares products for reuse, remanufacture, or recycling. Disassembly is a critical operation in this chain, separating products into reusable, remanufacturable, recyclable, and disposable parts. The disassembly process includes separation of functional parts from the rest of the product, the cleaning of usable parts, and quality inspection. Disassembly planning requires information from design, such as disassembly operations, sequencing, disassembly features, their relationships, and subassemblies. Designers need the cost of disassembly and available equipment to determine disassemblability of a design. This project provides an information model to exchange disassembly information among lifecycle applications, such as design, disassembly planning, and reverse logistics.

This project facilitates collaborative exploration of manufacturing alternatives by providing formal and model-based representations of engineering information beyond those typically available, including sustainability information such as disassembly. Semantically consistent engineering information simplifies lifecycle management, including capture of alternatives and incremental refinements that meet requirements and earlier engineering commitments. They also lead to more reliable interpretation of models across the product lifecycle due to more rigorous language semantics. Model-based techniques provide engineering-friendly languages, freeing engineers from learning the specifics of formal approaches, while still having their benefits. The project improves the power of model-based engineering languages, to enable different or overlapping aspects of manufacturing information to be developed separately, or built on each other, then assembled rapidly and flexibly with the aid of automated consistency checking.

\section{Objective(s)}

- Develop product and process modelling languages based on semantically precise foundation.

- Develop a disassembly information model and demonstrate it with test cases collected from industry.

\section{Accomplishments}

- Developed formal process semantics included in a major revision of the Business Process Model and Notation, Systems Modeling Language, and Unified Modeling Language, as standardized by the Object Management Group. This significantly improved the interoperability and precision of standards widely used in manufacturing.

- Developed a product modeling language combining the capabilities of ontology and model based approaches. This is expected to facilitate collaborative exploration of manufacturing design alternatives.

- Evaluated the Web Ontology Language for capturing manufacturing assembly structure and product modeling languages. This contributed to research on applying formally defined languages to manufacturing problems.

- Developed a draft disassembly model with features, tolerance, work piece, equipment, and workflow. This will improve the reliability of exchanging disassembly information among lifecycle applications, such as disassembly planning and reverse logistics. 


\section{Planned Future Accomplishments}

- Develop a process modeling language combining the capabilities of ontology and model-based approaches, and integrate it with the product modeling language.

- Extend the product modeling language to capture testing and evaluation of product designs.

- Develop a prototype modeling system to demonstrate the usage of the product and process modeling languages.

- Document and demonstrate the disassembly model. The model enables developing new software systems of design for eco-friendly products for reuse, recycle, and remanufacturing. The model also provides a basis for reliable data exchange of disassembly information among design, disassembly planning, and reverse logistics in product lifecycle applications.

\section{Customers and Collaborators}

John Deere; Caterpillar; Lockheed Martin; Raytheon; The Boeing Company; Airbus; International Business Machines; SAP; Oracle; Dell Computers; U.S. National Aeronautics and Space Administration; Object Management Group; Georgia Institute of Technology; Korea Advanced Institute of Science and Technology; Texas Tech University; University of Toronto; San Jose University; University of Miami 


\section{Long Term Knowledge Retention}

\section{Challenge/Problem Addressed}

ack of an infrastructure for archiving digital representation of manufactured artifacts impedes the ability to retrieve and use prior engineering knowledge. The nature of product and process information must be characterized to allow developing methodologies for sustaining long-term usability of engineering information, and defining metrics for digital preservation of engineering information from Computer-Aided Design (CAD), Computer-Aided Engineering, Computer-Aided Manufacturing, Product Lifecycle Management (PLM) and Life Cycle Assessment (LCA) and green (sustainable) manufacturing related tools.

This project focuses on two problem areas:

- Ingest of information packages into an archival system, because downstream archive functions (data management, access) depend on ingest quality, and ingest is arguably the most domain-sensitive archival function.

- Specification of engineering-specific descriptive metadata, to be used in conjunction with existing administrative and packaging metadata to aid in future access (query and retrieval).

\section{Objective(s)}

- Specify descriptive metadata for various partial descriptions of the product design to be archived, employing an ontological multi-layered modeling approach.

- Use the structure specified in the "Technical Data Packages" US Military Standard (MIL-STD-31000) to guide the descriptive metadata partitioning.

- Predict future access requirements for managing archived digital engineering objects by collecting use cases and developing access scenarios.

\section{Accomplishments}

- Developed a ship gate valve maintenance long-term archiving demonstration scenario using a variety of information types, some provided by the Navy. The scenario is outlined in a document containing UML diagrams and will serve as a roadmap for a proof-ofconcept demonstration.

- Proposed an initial architecture for ingest of a product model information package employing ontologicallyinspired representations for descriptive metadata and data elements inspired by the "Technical Data Packages" US Military Standard (MIL-STD-31000) for packaging metadata. The approach is documented in a completed proposal to the National Archives Records Administration as well as in an paper in preparation.

\section{Planned Future Accomplishments}

- Develop an ontologically specified schema representing engineering domain-specific descriptive information (metadata) to aid access to a product model data repository, with a documented procedure for populating the schema.

- Demonstrate product model data repository ingest and access to show the effectiveness of the proposed ingest approach and descriptive metadata schema.

\section{Customers and Collaborators}

US National Archives Records Administration; Naval Surface Warfare Center: Carderock Division; Automotive Industry Action Group; PDES, Inc.; University of Dijon 


\section{Simulation of Manufacturing Enterprises for Sustainability}

\section{Challenge/Problem Addressed}

odeling and Simulation $(\mathrm{M} \& \mathrm{~S})$ can play a major
role in the success of sustainable manufacturing through the ability to predict the impact of implementing certain facilities, processes, or product actions. M\&S has proven to be an effective tool for analyzing complex manufacturing systems. However, to utilize M\&S effectively, it is essential to develop a suitable infrastructure (such as measurements, standards, frameworks, models, and tools) that promotes interoperability and the application of simulation technologies for sustainable manufacturing, making their use transparent to the user. The need for effective $M \& S$ will continue to grow with increasing system complexity, promoting new requirements for addressing global sustainability and seamless exchange of vast quantities of information across the design, manufacturing, and distribution network.

\section{Objective(s)}

- Develop an essential and suitable infrastructure (such as measurements, standards, frameworks, models, and tools) that promotes interoperability and the application of simulation technologies for sustainable manufacturing.

- Develop an architecture for the integration of an identified set of modeling and simulation applications, engineering tools, standards and repositories of data.

- Implement reference information models supporting key extensions to simulation interfaces, evaluation metrics, modeling tools and reference data sets needed to support sustainability.

- Define taxonomy and templates supporting the characterization of key manufacturing unit processes that incorporate sustainability information that can be used to calculate performance metrics (process monitoring, control, and improvement.
- Capture best practices and performance metrics to evaluate objectively a company's progress and maturity level regarding the use of simulation in pursuit of sustainable manufacturing objectives.

- Provide focused demonstrations and case studies to illustrate extended simulations that support sustainable manufacturing.

\section{Accomplishments}

- Held symposium on "Modeling tools and metrics for sustainable manufacturing," American Society of Mechanical Engineers (ASME), International Design Engineering Technical Conferences (IDETC) and Computers and Information in Engineering (CIE) Conference (successful participation in 2009, regular session within CIE for 2010.) Developed a new track on "Modeling and Simulation for Sustainable Manufacturing" at the Winter Simulation Conference 2010. The symposiums are providing a needed forum to discuss, promote, and disseminate the new needs and requirements for $M \& S$ towards sustainable manufacturing.

- Published six conference papers, one journal paper and 1 NIST Internal Report (NISTIR). Publications disseminate results to various stakeholders. Publications market NIST subject-matter technical expertise, acknowledge NIST research interests, disseminate results to various stakeholders, and serve as a tool for promoting university and industry partnering.

- Initiated three industry collaborations on sustainable practices. Having industry partners establishes relevance and credibility of work and assists industry in establishing priorities regarding their sustainable best practices.

- Performed first phase combinatorial study of manufacturing process using simulation methods. This will result in more sustainable friendly factories to be built in reduced time and costs. 


\section{Planned Future Accomplishments}

- Define a preliminary structure of the simulation architecture and corresponding infrastructural components facilitating new trends toward science-based manufacturing and simulation. (Architecture)

- Develop preliminary information models supporting simulation for sustainable manufacturing. (Reference information models)

- Define a top-level taxonomy and classification for unit processes; define a generic template supporting science-based characterization of unit processes; create a repository for templates facilitating the characterization of unit processes, related reference information models and case studies. (Unit process)

- Determine initial set of manufacturing best practices; identify sustainability metrics for scoring such as energy consumption, types and quantities of material used, etc. (Sustainability maturity model)

- Identify new industrial relationships and potential collaborations; define initial extensions to simulation objectives, evaluation metrics, unit processes, modeling tools and reference data sets needed to support sustainability; demonstrate an initial set of extended case studies. (Industrial case studies and outreach)

\section{Customers and Collaborators}

Boeing Seattle, St. Louis; General Motors, Volvo, USCAR; Stanley Black and Decker (tentative); Unigraphics, Dassault systems - Delmia; Doyle Center, Pittsburgh; University of Maryland, Wichita State University, Washington State University, University of Michigan, Chalmers University, Sweden; Tampere University, Finland. 


\section{Supply Chain Integration}

Where once U.S. manufacturers might have built and controlled the transport for all the supplies needed for a product, they now rely on suppliers from all over the world. This increases flexibility and lowers costs but also opens up a slew of supply chain infrastructure problems, from tracking the supplies as they are shipped around the globe to quickly finding new suppliers whose goods can be integrated into an already-running production system. This program works with industry to establish the standards needed to permit compatibility across a massive, worldwide network of software applications covering supplier inventories, supply production schedules and shipment tracking information -- everything needed to get goods from a remote business to one's own.

\section{Challenge}

ron hroughout most of the 20th century, production and labor capabilities of individual original equipment manufacturers (OEMs) defined competitive advantage. combined capabilities of the suppliers that make up the OEMs' supply chain now defines competitive advantage is defined now by the. Therefore, the only way to improve competitive advantage is to improve those combined capabilities through better integration - that is information exchange across the supply chain. The challenge for this program is to develop and demonstrate an open, standards-based, testing and integration infrastructure that enables the automated exchange of information across the supply chain. Since every supplier can benefit from such an infrastructure, no individual supplier or OEM will commit the substantial resources necessary for its development and deployment. In addition, historically, the private sector has not invested heavily in such work because of (1) the difficulty assessing its potential costs and benefits and (2) the high technical risk and market uncertainty.

\section{Overview}

T⿳亠口冋口巾 his program's business focus is two, critical, supplychain-management issues: supplier discovery and long-range logistics. Both of these issues involve a number of integration problems. The program's technical focus is on the development and testing of standards needed to address those problems. It collaborates with organizations, such as Open Applications Group, Inc. (OAGi), that develop domain standards. This collaboration yields architectures and tools to improve the creation, validation, and testing of those standards. The program also collaborates with organizations, such as OMG and W3C that develop knowledge representation standards. This collaboration ensures that issue-specific manufacturing knowledge can be represented using those standards. Finally, the program collaborates with users, vendors, and industrial consortia to define particular integration problems and to demonstrate/ implement the solutions to those problems.

\section{Key Accomplishments and Impacts}

- Completed pilot exercise demonstrating Materials Off-Shore Sourcing (MOSS) solution for long-range logistics. Based on exercise cost/benefit analysis, the Automotive Industry Action Group (AIAG) predicts more than $400 \%$ internal rate of return and less than a 3-month payback.

- Completed Naming Design Rules (NDR) testing of Open Applications Group's Integration Specification (OAGIS) 9.0 specifications, which define interoperability points between business applications. Numerous errors were found and corrected before public release, strengthening the creditability of 9.0 as an implementable solution, and increasing interoperability across additional capabilities, e.g., logistics, shopping, and payment services. 


\section{Future Directions and Plans}

- Extend long-range logistics project to include intracontinental movement of goods to extend and apply demonstrated benefits from off-shore supplier pilot to intra-continental suppliers

- Initiate new project on reverse logistics supply chains to support new initiative in sustainable manufacturing (for reuse, recycle, and remanufacturing)

- Develop and demonstrate new integration infrastructure to support supplier discovery by U.S. manufacturers

\section{Publications}

Vujasinovic, M., Ivezic, N., Kulvatunyou, B., Barkmeyer E., Missikoff, M., Taglino, F., Marjanovic, Z., Miletic, I., "A Semantic Mediation Architecture for Interoperable Supply Chain Applications," International Journal of Computer Integrated Manufacturing, 976-986, Volume 22, Issue 6, 2008.

Shin, J., Kim, J., Ivezic, N., "Application information mapping test: An efficient content-level semantic equivalence test procedure for B2B integration," International Journal of Computer Integrated Manufacturing, 13623052, Volume 22, Issue 10, 2009.

Vujasinovic, M., Ivezic, N., Kulvatunyou, B., Barkmeyer E., Missikoff, M., Taglino, F., Marjanovic, Z., Miletic, I., "Semantic Mediation for Standard-Based B2B Interoperability," IEEE Internet Computing, 52-63, Volume 14, Issue 1, 2010.

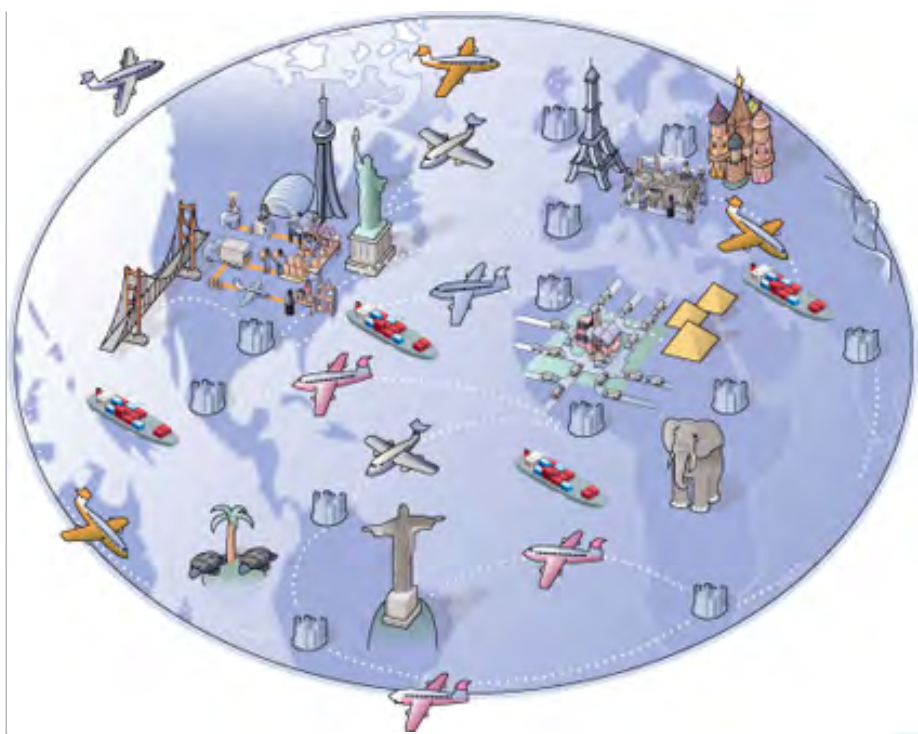

\section{Contact Information:}

Albert Jones, Program Manager

(301) 975-3554

albert.jones@nist.gov 


\section{Recognition and Awards}

\section{Board Membership}

\begin{tabular}{|l|l|}
\hline Staff & Board Membership \\
\hline Albert Jones & $\begin{array}{l}\text { - Member of Advisory Board Engineering Dept, Loyola University } \\
\text { - Member of Enterprise Architecture Board, Penn State University }\end{array}$ \\
\hline
\end{tabular}

\section{Leadership}

\begin{tabular}{|c|c|}
\hline Staff & Leadership \\
\hline Evan Wallace & $\begin{array}{l}\text { - Co-Chair - OMG Ontology Platform Special Interest Group : since it's chartering - now } \\
\text { - Co-Chair - OMG Ontology Definition Metamodel Finalization Task Force, until Sept } \\
2008 \text { when the group disbanded after completing work leading to the formal ODM } \\
\text { specification } \\
\text { - Co-Chair - OMG Ontology Definition Metamodel Revision Task Force : } \\
\text { Sept } 2008 \text { - now } \\
\text { - NIST representative - W3C OWL (Web Ontology Language) Working Group : which } \\
\text { produced a Recommendation for a revision of OWL called OWL } 2\end{array}$ \\
\hline Albert Jones & $\begin{array}{l}\text { - Government representative to the international Intelligent Manufacturing } \\
\text { Systems Program }\end{array}$ \\
\hline Edward Barkmeyer & $\begin{array}{l}\text { - Chairman of the OMG Finalization Task Force for the EXPRESS Metamodel } \\
\text { - Standard Chair of the Revision Task Force for the ISO/OMG Common Warehouse } \\
\text { Metamodel }\end{array}$ \\
\hline Nenad Ivezic & $\begin{array}{l}\text { - Member of the International Program Committee for the Interoperability for Enterprise } \\
\text { Systems and Applications (I-ESA) Conference 2008, } 2009 \\
\text { - Member of the International Program Committee for the eChallenges } \\
\text { Conference 2008, } 2009\end{array}$ \\
\hline
\end{tabular}

\section{Excellence}

\section{Staff $\quad$ Excellence Recognized}

Susanne Müller $\quad$ Certificate of Appreciation for M-19: Materials Off-Shore Sourcing (MOSS) Project

Peter Denno $\quad$ - AIAG Outstanding Technical Achievement Award, 2008 and 2009

Nenad Ivezic $\quad$ - Invited expert to the Global eBusiness Interoperability Test Bed Methodologies (GITB) project of the European Standardization Committee (CEN), 2009 


\section{Supply Chain Integration Projects}

\section{Increase Quality of Standards}

\section{Challenge}

ost supply chain interface standards have been $\checkmark$ created as XML (Extensible Markup Language) schemas. The standards development organizations (SDOs) responsible for creating those standards have published a set of guidelines, called naming and design (NDR) rules. These guidelines, which govern both the creation and use of these standards, vary considerably from one organization to another. Additionally, they were published originally in English text, which made testing very difficult. The early challenges for this project were to develop computer interpretable rules and a set of tools that can be used to test the standards against these new computational rules. MSID completed a project to convert the English text into computer interpretable rules and built associated tools to test the standards against these rules.

It is likely that the next generation of existing supply chain standards will be developed using a more formal modeling language, like Web Ontology Language $(\mathrm{OWL})$ or First Order Logic (FOL). Manufacturers will likely use these languages, or others, to capture business rules/processes. It is important to capture them, because they form a major part of the context in which integration testing will take place. The current challenge for this project is to ensure that these languages are capable of modeling manufacturing information and manufacturing rules/processes.

\section{Overview}

T his project has been collaborating with two SDOs to verify conformance of their supply chain standards to their NDRs: United Nations Center for Trade Facilitation and Electronic Business (UN/CEFACT) and The Open Applications Group Integration Specification (OAGIS). UN/CEFACT published a number of E-Commerce related standards. This verification was done using a collection of software testing tools that are now available as web services. OAGIS published a collection of interface standards used by both the aerospace and automotive industries. The project has also been working with OMG and W3C to publish a number of important standards.

\section{Accomplishments}

- Completed NDR testing of OAG 9.0, found many errors and corrected them before public release, strengthening the creditability of 9.0 as an implementable solution, and increasing interoperability across additional capabilities, e.g., logistics, shopping, and payment services.

- Completed six interoperability exercises for SYSML (Systems Modeling Language) and AP233. NIST provided the Validator, which provides a key capability for these exercises. These exercises provide a foundation for interoperability between applications that implement these two standards.

\section{Planned Future Accomplishments}

- Complete development of testing Web Service as a readily accessible service for industry and standards development organizations to access.

\section{Collaborators and Partners}

OAGi, UN/CEFACT, OMG, W3C 


\section{Reduce Shipping Delays and Costs in Long- distance Supply Chains}

\section{Challenge}

tudies conducted by the Automotive Industry Action - Group (AIAG) strongly suggested that the existing paper-based information exchange and communication systems have caused huge problems in the mangement of long-distance supply chains. Those studies concluded that a completely paperless system could (1) reduce shipment delays, inventory holdings, flow disruptions, and logistics costs, and (2) improve compliance with Customs-Trade Partnership Against Terrorism and World Customs Organization standards for secure trade, and increase stability and predicability of material flows. The challenge of this project was to develop and demonstrate such a paperless system.

\section{Overview}

\begin{abstract}
A IAG's Material Off-Shore Sourcing (MOSS) project was designed to improve the business processes and information exchanges that drive the intercontinental shipment of goods. Over the last five years of the project, the stakeholders involved in these shipments have included customer, supplier, carriers, third party logistics providers (3PLs), freight forwarders, customs brokers, and government agencies. In FY2007, AIAG asked MSID researchers to participate in the MOSS project. NIST worked with several of these stakeholders and a number of software vendors to develop the much needed paperless system. That system would include standardized Electronic Data Interchange (EDI) message definitions, a standardized encoding of the information content of these messages, guidelines for how to use the messages, and, methods and /tools for automatic generation of all required paper documents from these messages. A demonstration of the systems was to be conducted on a real shipment of batteries from Korea to the United States, with a subsequent analysis to measure the actual costs and benefits.
\end{abstract}

\section{Accomplishements}

- Completed development of the paperless system that addressed the challenges from long-distance supply chain identified above.

- Completed conformance testing of software vendor implementations against the EDI messages. This was necessary for vendors to particiapte in the pilot exercise.

- Completed pilot exercise and subsequenet cost/benefit analysis. The anlysis showed an internal rate of return gretaer than $400 \%$ with a payback period for participating stakeholders of less than three months.

\section{Planned Future Accomplishments}

- Complete second pilot from the EU to the US to derive the same benefits gleaned from the Korea-toUS pilot.

- Complete Cost/benefit analysis for second pilot to quantify the benefits from the preceding activity.

- Extend MOSS solutions to intra-continental logistics to derive the same benefits gleaned from the Koreato-US pilot.

\section{Collaborators}

OMG, Lockheed-Martin, Dept. of Defense, Lockheed-Martin, all major UML tool providers, users of OMG Meta-Object Facility (MOF -technology tools (e.g., UML tools) 


\section{Facilitate Discovery of New Partners in Virtual Supplier Networks}

\section{Challenge}

D igid supply chain structures are giving way to virtual supply networks of collaborative partnerships sometimes call supplier discovery networks. These networks will develop rapidly in response to market opportunities; and, they will disband just as quickly when those opportunities disappear. They will require the Original Equipment Manufacturers (OEMs) to have real-time knowledge of the capabilities and capacities of all suppliers. Moreover, they will require suppliers to have real-time access to and a thorough understanding of product requirements and engineering changes. A critical requirement for these forming collaborative partnerships is supplier discovery --- the ability for OEMs to find suppliers who have the capabilities to meet their design requirements. The challenge for this project is to develop an integrating infrastructure that automates, to the extent possible, the process of supplier discovery.

\section{Overview}

his project was established under MEL's competitive Exploratory Projects program funding. This funding is used to: perform preliminary work as the foundation of a future initiative proposal; develop a project that will eventually be funded by sources outside of MEL; explore the possibility of a new MEL Program; or start work related to an existing program that may be eventually incorporated into that program. Initial discussion with DSN Innovations, Pittsburgh, PA, and Army's Picatinny Arsenal, NJ indicated a need for such an integration infrastructure that facilitates internet-based discovery of suppliers. Such an infrastructure will include standard models for representing requirements and capabilities, tools that enable OEMs and suppliers to register those requirements and capabilities, and methods and tools that allow designers to find potential suppliers whose capabilities match their requirements automatically. This planning project was initiated last spring. It will create a technical plan for developing and demonstrating such an infrastructure.

\section{Accomplishments}

- Completed detailed project plan that provides the research roadmap for the project.

- Completed a review of matchmaking technologies that are a fundamental part of the research roadmap.

- Completed review of several on-line marketplaces. This review will provide a state-of-the-practice understanding for the project.

\section{Planned Future Accomplishments}

- Select candidate matching techniques to improve automated efficiencies of supplier discovery.

- Develop experimental plan to choose from among candidates to select the most effective technique.

- Pilot test the selected candidate with stakeholder industrial collaborators.

\section{Collaborators}

University of Texas, Others to be determined 


\section{Improve Content Testing for Manufacturing Integration Solutions}

\section{Challenge}

s upply chain partners still face enormous technical integration problems exchanging manufacturing messages using existing supply chain integration standards and the commercial software solutions that implement them. Currently available testing tools can verify conformance of the form and structure of the messages to the form and structure specified in the underlying standards. But, these tools cannot determine whether the content of messages is "correct." The challenge of this project is to develop new approaches for testing content for an individual message and for a flow of messages associated with a particular business process. This requires a new kind of testing, which we call content testing.

\section{Overview}

Ontent testing examines the manufacturing data itself and the context in which it is being used.

Whether the manufacturing data has a well-defined meaning may depend on the context in which it is used. For individual messages, that context can often be expressed as one (or more) constraints(s). For these situations, we propose a constraint-based testing approach. This approach attempts to constrain the values of the data by applying the underlying standard. We identified a number of such constraints within the standard, developed a suite of associated tools, and tested those tools against a small subset of Open Applications Group (OAG) specifications.

When dealing with a collection of interdependent messages, this testing approach is not sufficient. This situation arises frequently when the manufacturing scenario involves the execution of a complicated business process - the Materials Off-Shore Sourcing MOSS project is one example. To address content testing in such scenarios, we propose a new architecture that uses ontologies to capture business rules and other types of necessary knowledge and a new type of reasoner to execute the actual content testing of the messages.

\section{Accomplishments}

- Developed ontology-based content testing architecture that will be the foundation for all of our future testing activities.

- Completed content-checking tools for initial class of constraints. These will be used to conduct testing on selected OAG specifications.

- Demonstrated use of semantic mediation in Businessto-Business (B2B) interoperability testing. Semantic mediation is the fundamental technology needed for successful interoperability testing.

\section{Planned Future Accomplishments}

- Demonstrate new tools on selected supply chain standards to apply the results from the previous accomplishment against real-time customer manufacturing data.

- Develop initial ontology-based testing tools and associated models to verify correctness.

- Demonstrate tools and models on selected MOSS messages to expand the applied usefulness for intracontinental supply chains.

\section{Customers and Collaborators}

Open Applications Group, Automotive Industry Action Group, University of Maryland Baltimore Campus 
Appendix 


\section{PED Staff Recognition 2009-2010}

\section{Board Memberships}

\begin{tabular}{|c|c|}
\hline Staff & Board Membership \\
\hline Estler, Tyler & - BIPM Director's Advisory Group on Uncertainty, Sevres, France \\
\hline Phillips, Steven & $\begin{array}{l}\text { - Association of Coordinate Metrology, Canada } \\
\text { - Board of the North American Coordinate Metrology Association } \\
\text { - Board for US Standard Technical Advisory Group }\end{array}$ \\
\hline Postek, Michael & $\begin{array}{l}\text { - SPIE Advanced Lithography Advisory Board } \\
\text { - SCANNING Editorial and Advisory Boards } \\
\text { - SPIE/SCANNING Conference Co-chairman and organizer } \\
\text { - SPIE Instrumentation, Metrology and Standards for Nanomanufacturing Conference } \\
\text { Co-chairman and organizer } \\
\text { - Nanotechnology Briefs Board of Directors }\end{array}$ \\
\hline Potzick, James & - SEMI Standards Microlithography Committee \\
\hline Silver, Richard & $\begin{array}{l}\text { - SPIE Advanced Lithography Executive Committee, 2006-2010 } \\
\text { - North American Regional Standards Committee, SEMI Standards } \\
\text { - Advanced Metrology Advisory Group, SEMATECH } \\
\text { - Editorial Board, Nanotechnology E-Bulletin } \\
\text { - NIST Patent Review Committee, 2007-2009 }\end{array}$ \\
\hline Vladar, Andras & $\begin{array}{l}\text { - ISMI/SEMATECH Advanced Lithography Advisory Board } \\
\text { - SCANNING Editorial and Advisory Boards } \\
\text { - ITRS International Road Map for Semiconductor Technology -Metrology Section }\end{array}$ \\
\hline
\end{tabular}




\section{Conference Chairmanships}

\begin{tabular}{|c|c|}
\hline Staff & Conference Chairmanships \\
\hline Dixson, Ronald & - 2nd Annual Tri-National Workshop on Standards for Nanotechnology NIST, Feb. 2008 \\
\hline Postek, Michael & $\begin{array}{l}\text { - Instrumentation, Metrology and Standards for Nanomanufacturing. SPIE 2008, San Diego CA. } \\
\text { - Instrumentation, Metrology and Standards for Nanomanufacturing. SPIE 2009, San Diego CA. } \\
\text { - Instrumentation, Metrology and Standards for Nanomanufacturing. SPIE 2010, San Diego CA. } \\
\text { - Electron/Instrument Interaction Modeling in the Scanning Electron Microscope XII. A NIST Spon- } \\
\text { sored Workshop, } 2008 \text { SCANNING, April, } 2008 .\end{array}$ \\
\hline Silver, Richard & $\begin{array}{l}\text { - Modeling Aspects in Optical Metrology, SPIE International Conference, 2007, } 2009 \\
\text { - Nanotechnology Working Group, Co-chair, 2005-2009, SPIE sponsored sessions. } \\
\text { - SEMI Standards Microlithography Committee, Co-chair, 2004-2009 }\end{array}$ \\
\hline Vladar, Andras & - XII. 2008 SCANNING, NIST, Gaithersburg April, 2008 \\
\hline Stoup, John & $\begin{array}{l}\text { - ASPE Spring Topical Meeting on Mechanical Metrology and Measurement Uncertainty. April 4-6, } \\
\text { 2009, Albuquerque, NM }\end{array}$ \\
\hline
\end{tabular}

\section{Session Chairmanships}

\begin{tabular}{|c|c|}
\hline Staff & Session Chairmanships \\
\hline Orji, George & $\begin{array}{l}\text { - Instrumentation, Metrology and Standards for Nanomanufacturing. SPIE 2009, San Diego CA. } \\
\text { - Instrumentation, Metrology and Standards for Nanomanufacturing. SPIE 2010, San Diego CA. }\end{array}$ \\
\hline Phillips, Steven & $\begin{array}{l}\text { - NACMA: Coordinate Metrology, Mexico } 2009 \\
\text { - ACMC: CMMs, Windsor CA, } 2008\end{array}$ \\
\hline Potzick, James & $\begin{array}{l}\text { - SPIE Photomask Technology } 2009 \\
\text { - SPIE Photomask Technology } 2010\end{array}$ \\
\hline Silver, Richard & $\begin{array}{l}\text { - SPIE Advanced Lithography, metrology sessions chair, 2004-2010 } \\
\text { - Instrumentation, Metrology and Standards for Nanomanufacturing, Program Committee }\end{array}$ \\
\hline Song, John & $\begin{array}{l}\text { - Advanced surface evaluations for the Ninth International Symposium on Measurement } \\
\text { Technology and Intelligent Instruments (ISMTII 2009), Sat Petersburg, Russia, June, } 29 \text { to July 3, } \\
2009 . \\
\text { - Traceability in other areas for the IMEKO TC8 } 2008 \text { conference, November 2008. Torino, Italy. } \\
\text { - Advanced surface metrology for the } 4^{\text {th }} \text { International Symposium on Precision Mechanical } \\
\text { Measurements (ISPMM 2008), Hefei, China, September } 2008 .\end{array}$ \\
\hline Villarrubia, John & $\begin{array}{l}\text { - Chair of Modeling session and Metrology session at Scanning, 2008, Monterery, CA. } \\
\text { - Chair of Modeling session, Scanning, } 2009 . \\
\text { - Chair of Modeling session, Scanning, } 2010 .\end{array}$ \\
\hline Vladar, Andras & - Charged Particle Metrology XII. A NIST Sponsored Workshop, 2008 SCANNING, April, 2008. \\
\hline
\end{tabular}




\section{Technical Workshops and Short Courses Presented}

\begin{tabular}{|c|c|}
\hline Staff & Workshops/Short Courses \\
\hline Dixson, Ronald & $\begin{array}{l}\text { - NIST-SPIE sponsored workshop in conjunction with SPIE Advanced Lithography Con- } \\
\text { ference, March 2008, } 2009\end{array}$ \\
\hline Orji, George & $\begin{array}{l}\text { - Invited short course: "Introductory Metrology" International SEMATECH Manufactur- } \\
\text { ing Initiative Symposium on Manufacturing Effectiveness. Austin TX, } \\
\text { October 21-24, } 2008 \\
\text { - Moderator: Panel Discussion on "Contour Metrology" at the SPIE Advance Lithography } \\
\text { Conference } 2009 \\
\text { - Moderator: SPIE Panel discussion on Reference Metrology (with Ronald Dixson). SPIE } \\
\text { Advanced Lithography Conference. San Jose CA. February 28th } 2008\end{array}$ \\
\hline Postek, Michael & $\begin{array}{l}\text { - Scanning Microscopy in Forensic Science (with Frank Platek, USDA and Dennis Ward, } \\
\text { FBI). Scanning 2009, Monterey, CA. } \\
\text { - Scanning Microscopy in Forensic Science (with Frank Platek, USDA and Michael } \\
\text { Trimpe. Scanning 2010, Monterey, CA. }\end{array}$ \\
\hline Song, John & $\begin{array}{l}\text { - The NIST workshop for SRM } 2461 \text { standard casings was hold in Largo, FL, } \\
\text { January 8, } 2008 . \\
\text { - The first workshop for NIST National Ballistics Imaging Comparison (NBIC) project, } \\
\text { July 8, 2008, Orlando, FL. } \\
\text { - The second workshop for NIST National Ballistics Imaging Comparison (NBIC) proj- } \\
\text { ect, January 8, 2009, New Orleans. }\end{array}$ \\
\hline Potzick, James & $\begin{array}{l}\text { - Short course Nanoscale metrology--theory and practice (with Brian Grenon, Grenon } \\
\text { Consulting, Inc.) at BACUS } 2007 \text { and } 2008 .\end{array}$ \\
\hline
\end{tabular}




\section{Leadership Demonstrated}

\begin{tabular}{|c|c|c|}
\hline Staff & Leadership Demonstrated & \\
\hline Dagata, John A. & \multicolumn{2}{|c|}{$\begin{array}{l}\text { - Organizing committee, NSF-sponsored workshop on Grand Challenges for Bio-Nano Inte- } \\
\text { grated Manufacturing, Arlington VA April 14-16, 2008.Organizing committee and keynote } \\
\text { speaker, } 2008 \text { International Workshop on Tip-based Nanofabrication, National Taiwan } \\
\text { University of Science and Technology, Taipei, Taiwan, October 19-21, } 2008 .\end{array}$} \\
\hline Dixson, Ronald & \multicolumn{2}{|c|}{ - Chair of ISO/TC201/SC9/SG3 } \\
\hline Estler, Tyler & \multicolumn{2}{|c|}{$\begin{array}{l}\text { - Chairman ASME B89.7.4 Measurement Risk Analysis } \\
\text { - Chairman JCGM WG1 S/C } 3 \text { Conformity Assessment } \\
\text { - Chairman, Scientific and Technical Committee P (Precision Engineering and Metrology), } \\
\text { CIRP - the International Academy for Production Engineering }\end{array}$} \\
\hline Phillips, Steven & \multicolumn{2}{|c|}{$\begin{array}{l}\text { - NIST Representative to SIM (North \& South American Metrology) Coordinator, Mexico } 2009 \\
\text { - Chair, ASME B89.4 Division on Coordinate Metrology } \\
\text { - Chair, ASME B89.4.7 Working Group on Measurement Uncertainty } \\
\text { - Chair, ASTM E57 Working Group on Short Range Laser Scanners } \\
\text { - Subject Matter Expert \#1 for US to ISO TC213 WG10 on Coordinate Metrology } \\
\text { - Subject Matter Expert \#1 for US to ISO TC213 WG4 on Measurement Uncertainty }\end{array}$} \\
\hline Postek, Michael & \multicolumn{2}{|c|}{ - National Science Foundation COV CMMI } \\
\hline Potzick, James & \multicolumn{2}{|c|}{$\begin{array}{l}\text { - Convener and leader, SEMI Standards Task Force Terminology for } \\
\text { Microlithography Metrology } \\
\text { - Leader Nano1 international comparison of linewidth measurements for BIPM. }\end{array}$} \\
\hline Renegar, Brian & \multicolumn{2}{|c|}{ - Task force chair ASME B46 Surface Texture revision } \\
\hline Sawyer, Daniel & \multicolumn{2}{|c|}{$\begin{array}{l}\text { - Chairman ASME B89.1. Oil and Survey Tapes } \\
\text { - Chairman ASME B89.4.19 Laser Trackers }\end{array}$} \\
\hline Silver, Richard & \multicolumn{2}{|c|}{$\begin{array}{l}\text { - SPIE Advanced Lithography, Organizing committee, 2001-2010 } \\
\text { - Instrumentation, Metrology and Standards for Nanomanufacturing, Program Committee } \\
\text { - Instrumentation, Metrology and Standards for Nanomanufacturing, } \\
\text { Organizing Committee, 2007-2010 } \\
\text { - Modeling Aspects in optical Metrology, Organizing Committee, 2008-10. } \\
\text { - SEMI Microlithography Committee Chair } \\
\text { - SEMI Standards two-dimensional grids task force leader } \\
\text { - SEMI Standards overlay metrology task force leader } \\
\text { - Overlay Metrology Advisory Group, SEMATECH } \\
\text { - Defect Metrology Advisory Group, SEMATECH } \\
\text { - Special Appt. to the Faculty, Dept of Physics, Univ. of Maryland }\end{array}$} \\
\hline Sharkarji, Craig & - Chairman, ASME B89.4.1 & \\
\hline
\end{tabular}




\begin{tabular}{|c|c|}
\hline Staff & Leadership Demonstrated \\
\hline Song, John & $\begin{array}{l}\text { - Post-Doc Research Adviser of NRC/NAS for Dr. Al Hilton's research project entitled "Ballis- } \\
\text { tics Identification using Topography Measurements and Correlations" (2007-present). } \\
\text { - Official Observer for the CIPM (BIPM) Consultative Committee for Mass and Related Quan- } \\
\text { tities - Working Group on Hardness (CCM-WGH). } \\
\text { - Council Member for the ICMI (International Committee on Measurements and } \\
\text { Instrumentation) } \\
\text { - Committee Member for International Measurement Confederation (IMEKO) TC } 5 \text { (Hardness } \\
\text { Measurement) } \\
\text { - International Committee Member for the ISMTII (International Symposium on Measurement } \\
\text { Technology and Intelligent Instruments) } \\
\text { - International Committee Member for the ISIST (International Symposium on Instrumentation } \\
\text { Science and Technology) } \\
\text { - International Committee Member for the ISPMM (International Symposium on Precision } \\
\text { Mechanical Measurements); }\end{array}$ \\
\hline Stanfield, Eric & $\begin{array}{l}\text { - Chairman ASME B89.1.19 Master Balls } \\
\text { - MEL Fuel Cell Project Manager for DOE Hydrogen Program sponsored Multi-Year Project } \\
\text { "Metrology for Fuel Cell Manufacturing." }\end{array}$ \\
\hline Stone, Jack & $\begin{array}{l}\text { - BIPM, Consultative Committee for Length (CCL), Sevres, France } \\
\text { - Chairman, ad hoc Working Group on Unstabilized Lasers in the Mise en Pratique } \\
\text { of the CCL } \\
\text { - Working Group on Dimensional Metrology of the CCL } \\
\text { - ASME B89.1.8 Laser Interferometers }\end{array}$ \\
\hline Vorburger, Ted & - SME \#1 ISO TC213 WG16 Areal and profile surface texture \\
\hline Vladar, Andras & $\begin{array}{l}\text { - ISO TC } 204 \text { SEM Image Sharpness Metrology Standard, NIST } 2009 \\
\text { - ISO Nanoparticle Metrology Standards, Tel Aviv } 2009 \text { - } 2010\end{array}$ \\
\hline Everett, Dennis & $\begin{array}{l}\text { - API Sub-Committee } 5 \text { Member - Tubular Goods } \\
\text { - ASME B1 Main Committee Member/NIST Liason } \\
\text { - ASME B1.2 Member - Gages and Gaging for Unified Inch Screw Threads }\end{array}$ \\
\hline
\end{tabular}




\section{Manuscript Reviewer}

\begin{tabular}{|c|c|}
\hline Staff & Manuscript Reviewer \\
\hline Attota, Ravikiran & - WEAR, 2008 \\
\hline Borchardt, Bruce & $\begin{array}{l}\text { - Journal: Measurement Science and Technology } 2007 \\
\text { - PED WERB sponsor }\end{array}$ \\
\hline Estler, Tyler & $\begin{array}{l}\text { - Annals of the CIRP - The International Academy of Production Engineering, } 2008 \text { - multiple } \\
\text { paper reviews } \\
\text { - Measurement Science and Technology, } 2007\end{array}$ \\
\hline Phillips, Steven & - Journal of Manufacturing Science and Engineering \\
\hline Postek, Michael & $\begin{array}{l}\text { - Electron, Ion, and Photon Beam Technology and Nanofabrication/JVST, } 2009 . \\
\text { - SCANNING } 2009 \\
\text { - SCANNING } 2008\end{array}$ \\
\hline Silver, Richard & $\begin{array}{l}\text { - JVST B } \\
\text { - Journal of Chem Physics }\end{array}$ \\
\hline Vladar, Andras & $\begin{array}{l}\text { - SCANNING, } 2005 \text { - } 2010 . \\
\text { - UltraMicroscopy } 2005 \text { - } 2010\end{array}$ \\
\hline
\end{tabular}




\section{Excellence Recognized (Awards)}

\begin{tabular}{|c|c|}
\hline Staff & Excellence Recognized \\
\hline Attota, Ravi & - Nano 50 Award for Scatterfield Optical Microscopy \\
\hline Blackburn, Chris & $\begin{array}{l}\text { - The NIST Judson C. French Award, for significant improvements in accuracy } \\
\text { and extensions of range in the calibration of long length standards }\end{array}$ \\
\hline Borchardt, Bruce & $\begin{array}{l}\text { - The NIST Judson C. French Award, for significant improvements in accuracy } \\
\text { and extensions of range in the calibration of long length standards }\end{array}$ \\
\hline Estler, Tyler & $\begin{array}{l}\text { - The NIST Judson C. French Award, for significant improvements in accuracy } \\
\text { and extensions of range in the calibration of long length standards }\end{array}$ \\
\hline Estler, Tyler & $\begin{array}{l}\text { - 2009. ASME's Certificate of Achievement" for outstanding national } \\
\text { standards work }\end{array}$ \\
\hline Phillips, Steven & - DOC Gold Medal, for leadership in international standards \\
\hline Phillips, Steven & $\begin{array}{l}\text { - The NIST Judson C. French Award, for significant improvements in accuracy } \\
\text { and extensions of range in the calibration of long length standards }\end{array}$ \\
\hline Phillips, Steven & $\begin{array}{l}\text { - 2009. ASME's Certificate of Achievement" for outstanding national } \\
\text { standards work }\end{array}$ \\
\hline Potzick, James & - Elected Fellow of SPIE \\
\hline Sawyer, Daniel & $\begin{array}{l}\text { - The NIST Judson C. French Award, for significant improvements in accuracy } \\
\text { and extensions of range in the calibration of long length standards }\end{array}$ \\
\hline Sawyer, Daniel & $\begin{array}{l}\text { - NIST Bronze Medal, for the design and fabrication of an operating room com- } \\
\text { puter assisted orthopedic hip surgery artifact }\end{array}$ \\
\hline Shakarji, Craig & $\begin{array}{l}\text { - DOC Gold Medal, for leadership in international standards } \\
\text { - NIST Bronze Medal, for the design and fabrication of an operating room com- } \\
\text { puter assisted orthopedic hip surgery artifact }\end{array}$ \\
\hline Silver, Richard & $\begin{array}{l}\text { - Nano } 50 \text { Award for Scatterfield Optical Microscopy } \\
\text { - Elected Fellow of SPIE, } 2008 \\
\text { - DOC Silver Medal 2008, for development of optical methods in } \\
\text { overlay metrology. }\end{array}$ \\
\hline Stone, Jack & - DOC Silver Medal, for development of the NIST microfeature CMM probe \\
\hline
\end{tabular}




\section{Invited Presentations}

\begin{tabular}{|c|c|}
\hline Staff & Invited Presentations \\
\hline Attota, Ravikiran & $\begin{array}{l}\text { - Invited author: "Nanoscale measurements with a through-focus scanning optical microscope," } \\
\text { Future Fab, 30, pp 83-88, (2009). } \\
\text { - Invited presentation: "Nano-scale dimensional analysis with through-focus scanning optical } \\
\text { microscope imaging method", SME Nanomanufacturing, April 1-2, 2009, Minneapolis, MN }\end{array}$ \\
\hline Dixson, Ronald & $\begin{array}{l}\text { - "Reference Metrology in a Research Fab: The NIST Clean Calibrations Program,"SEMATECH } \\
\text { Advanced Metrology Advisory Group, Austin, TX, September 24, } 2008 . \\
\text { - "Traceable AFM Dimensional Metrology at NIST," NIST Workshop on Calibrations and Standards } \\
\text { For Nanomechanical Measurements, Gaithersburg, MD, June 16, } 2009 .\end{array}$ \\
\hline Orji, George & $\begin{array}{l}\text { - Keynote presentation: "Traceability: The Kay to Nanomanufacturing" Sixth International Confer- } \\
\text { ence on "Precision, Meso, Micro and Nano Engineering, Coimbatore, India December } 2009 \\
\text { - "Measurement traceability and quality assurance in a nanomanufacturing environment" Instru- } \\
\text { mentation, Metrology and Standards for Nanomanufacturing. SPIE 2009, San Diego CA. } \\
\text { - "NIST Traceable AFM Program" SEMATECH Advanced Metrology Advisory Group (AMAG) } \\
\text { meeting, Albany, September } 2009 . \\
\text { - "NIST Reference Metrology Program" - Global Collaboration on Reference Metrology - SPIE } \\
\text { - Advance Lithography Conference } 2009 \\
\text { - "NIST -SEMATECH CD-AFM RMS Project" Program Advisory Group (PAG) Meetings. } \\
\text { February } 2008 \\
\text { "Carbon Nanotubes as AFM tips" 2nd Annual Tri-National Workshop on Standards for } \\
\text { Nanotechnology February } 2008\end{array}$ \\
\hline Phillips, Steven & $\begin{array}{l}\text { - Keynote presentation, } 2009 \text { Quality Measurement Conference, May } 2009 \text { Orlando, FL. } \\
\text { - Invited talk, "Dimensional Measurement Traceabilty of 3D Imaging Data", SPIE, January 2009, } \\
\text { San Jose, CA. } \\
\text { - Invited talk, "Economics of Measurement Uncertainty and Tolerances", July, 2009, Peoria IL. } \\
\text { - Invited talk, "Laser Trackers and Standards", North American Coordinate Metrology Conference, } \\
\text { September 2009, Mexico. } \\
\text { - Invited Talk: "Laser Trackers: Testing and Standards", Manufacturing \& Measurement Conference, } \\
\text { Clearwater, FL, } 2008\end{array}$ \\
\hline
\end{tabular}




\begin{tabular}{|c|c|}
\hline Staff & Invited Presentations \\
\hline Postek, Michael & $\begin{array}{l}\text { - Measurement Science Addressing the EH\&S Aspects of Engineered Nanomaterials "Tiny Threats" } \\
\text { by the International Life Sciences Institute (ILSI). 2009. Tuscon, AZ. } \\
\text { - Measurements, Instrumentation and Standards for Nanotechnology. Society of Manufacturing } \\
\text { Engineers, 2008, Second Manufacturing Education Leadership Forum Long Island, NY. } \\
\text { - Nanotechnology: Important link between trees and airplanes. } 2008 \text { Forest Products Industry } \\
\text { National Meeting St. Louis MO. } \\
\text { - NANOTEX 2008. The Potentials of Helium Ion Microscopy for Semiconductor Process Metrology. } \\
\text { Dallas Texas. } \\
\text { - } 2008 \text { Advanced Curriculum Working Group: Nanotechnologies. Society of Manufacturing Engi- } \\
\text { neers Second Manufacturing Education Leadership Forum, Long Island, NY. } \\
\text { - } 2008 \text { Cellulose Nanocrystals the Next Big Nano-thing? SPIE Instrumentation, Metrology and Stan- } \\
\text { dards for Nanomanufacturing. August 2008 San Diego, CA. } \\
\text { - } 2008 \text { Helium lon Microscopy: Revolutionary Measurement Technology? Zeiss Laboratory open- } \\
\text { ing, Boston, MA } \\
\text { - } 2008 \text { NSF/Purdue University Workshop on Predictive Modeling "Predictive Modeling of Materials } \\
\text { at the Nanoscale" } \\
\text { - } 2008 \text { Nanotechnology Directions within the Dimensional and Mechanical Metrology Programs of } \\
\text { the NIST Manufacturing Engineering Laboratory for the Tri-National Workshop held at NIST } \\
\text { - } 2008 \text { Key Elements for the Future of Nanomanufacturing: Instrumentation, Metrology, and Stan- } \\
\text { dards at a workshop hosted by the Center for Hierarchical Manufacturing, an NSF Nanoscale } \\
\text { Science \& Engineering Center } \\
\text { - }\end{array}$ \\
\hline Potzick, James & $\begin{array}{l}\text { - Invited presentation: "Limits of optical dimensional metrology at the nano-scale," } \\
\text { 2nd Tri-National Workshop on Standards for Nanotechnology. }\end{array}$ \\
\hline Silver, Richard & $\begin{array}{l}\text { - Invited Plenary Presentation, "Improving Optical Measurement Accuracy using Multi-Technique } \\
\text { Nested Uncertainties", SPIE Advanced Lithography, } 2009 . \\
\text { - Invited Plenary Presentation, "STM Induced Surface Modification with Reactive Ion Etch Pattern } \\
\text { Transfer", EIPBN, Marco FL, } 2009 . \\
\text { - Invited Paper, "Angle-resolved Optical Metrology using Multi-Technique Nested Uncertainties", } \\
\text { SPIE Germany, } 2009 . \\
\text { - SEMATECH AMAG, two invited presentations on OCD limits and advanced optical overlay } \\
\text { metrology, March } 2009 . \\
\text { - SEMATECH DMAG, invited presentation on Advanced Optical Defect Metrology, March 2007, } \\
\text { March 2009. }\end{array}$ \\
\hline
\end{tabular}




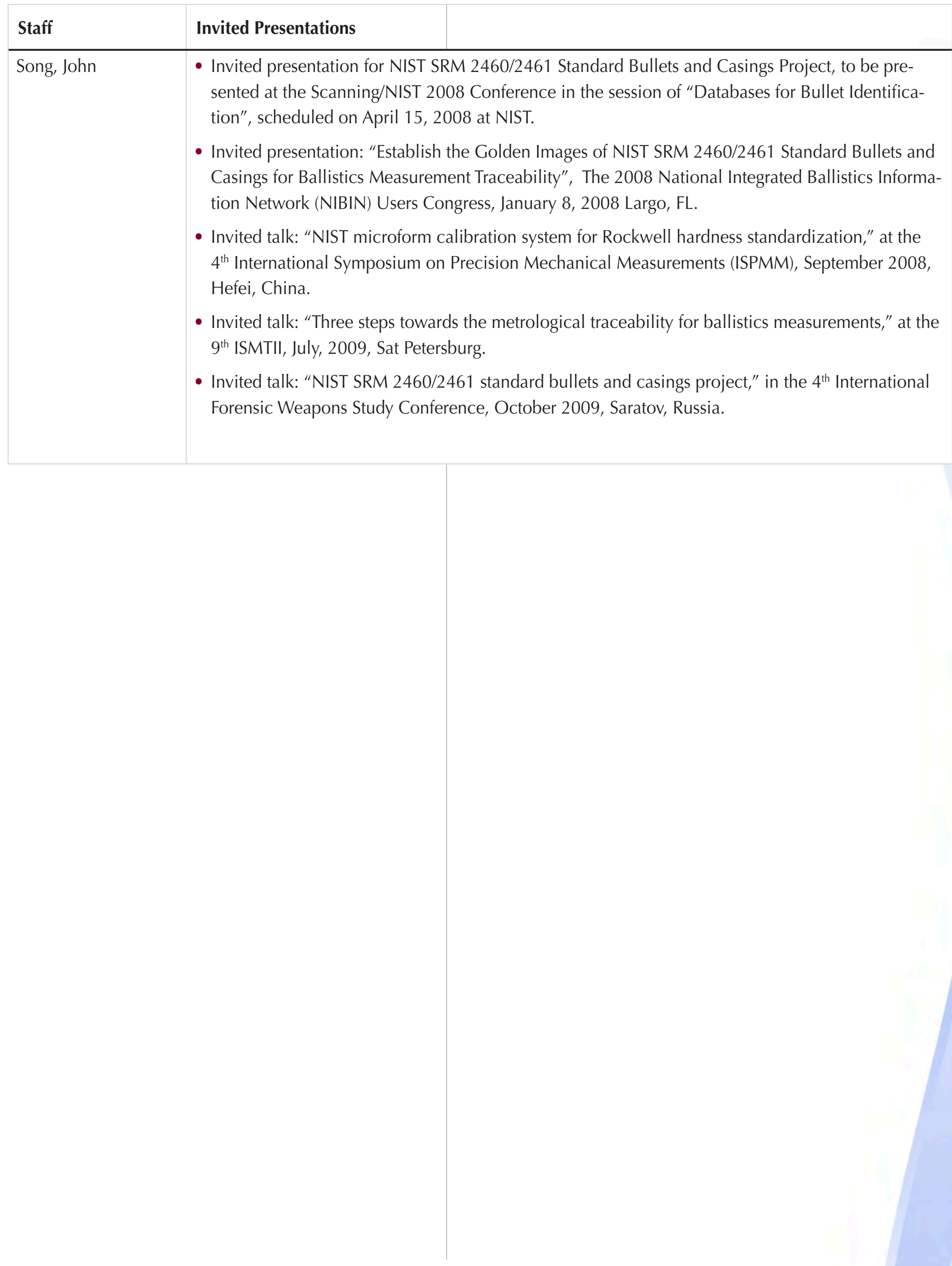




\section{Evidence of MEL Leadership (Since 2008)}

\section{Board Memberships}

\begin{tabular}{|l|l|}
\hline Staff & Board Membership \\
\hline Donmez, Alkan & $\begin{array}{l}\text { - Member, Industrial Advisory Board, MTConnect Institute } \\
\text { - Member, Industrial Advisory Board, Smart Machine Platform Initiative (SMPI) } \\
\text { - Member, Editorial Board of International Journal of Mechatronics and Manufacturing } \\
\text { Systems (IJMMS) } \\
\text { - Member, NIST Research Library Advisory Board; responsible for guiding the NIST } \\
\text { Fick, Steve }\end{array} \quad \begin{array}{l}\text { library for decisions about content and new acquisitions } \\
\text { - Member, NIST Assessment Review Board (ARB); responsible for conducting and } \\
\text { approving quality system reviews for the measurement services of each NIST division }\end{array}$ \\
\hline Harper, Kari & $\begin{array}{l}\text { Member, Board of Directors of the North American Manufacturing Research Institute } \\
\text { of the Society of Mechanical Engineering (NAMRI/SME) }\end{array}$ \\
\hline Ivester, Robert & $\begin{array}{l}\text { - Member, NIST Institutional Review Board (IRB); appointed by the NIST Director } \\
\text { - Member, American Society for Precision Engineering (ASPE) Board of Directors }\end{array}$ \\
\hline Nedzelnitsky, Victor & $\begin{array}{l}\text { Chair, NIST Museum Committee; responsible for the display and preservation of NIST } \\
\text { artifacts and instruments that have historical importance }\end{array}$ \\
\hline Rhorer, Richard &
\end{tabular}

\section{Leadership}

\begin{tabular}{|l|l|}
\hline Staff & Leadership \\
\hline Bruins, Jeanne & $\begin{array}{l}\text { - Participant in the year-long Administrative Professionals Career Development Program } \\
\text { sponsored by the Department of Commerce }\end{array}$ \\
\hline Cooke, April & - Member, ASTM Technical Committee F42 on Additive Manufacturing Technologies \\
\hline Donmez, Alkan & Secretariat, ISO TC39/SC2 Standards Committee "Test Conditions for Machine Tools" \\
- & Chair, ANSI/ASME U.S. Technical Advisory Group to ISO/TC39 - Machine Tools \\
- & Member, ANSI/ASME B5 Standards Committee "Machine Tools - Components, Ele- \\
& ments, Performance, and Equipment" \\
- & Member, ANSI/ASME B5/TC52 Standards Committee "Machine Tool Performance" \\
- Member, ANSI/ASME B5/TC56 Standards Committee "Information Technology for & Machine Tools" \\
- Member, Organizing Committee of the 3rd International Conference on Micromanu- \\
facturing [2008] \\
- Member, International Steering Committee of the Lamdamap 9th International Confer- \\
ence on Machine Metrology [2009]
\end{tabular}




\begin{tabular}{|c|c|}
\hline Staff & Leadership \\
\hline Evans, Dave & $\begin{array}{l}\text { - Chair of U.S. Delegation for the ISO TC108 Standards Committee "Mechanical Vibra- } \\
\text { tion and Shock" } \\
\text { - Chair of U.S. Delegation for the ISO TC108/SC3 Standards Committee "Use and Cali- } \\
\text { bration of Vibration and Shock Instrumentation" } \\
\text { - Vice-Chair, ANSI/ASA S2 Standards Committee "Mechanical Vibration and Shock" } \\
\text { - Chair, ANSI/ASA S2/WG2 Standards Committee "Terminology" } \\
\text { - Chair, ANSI/ASA S2/WG5 Standards Committee "Calibration and Use of Instruments" }\end{array}$ \\
\hline Griesmann, Ulf & $\begin{array}{l}\text { - Member, Optics and Electro-Optics Standards Council (OEOSC), a forum of techni- } \\
\text { cal experts that provides guidance on behalf of the U.S. for national and international } \\
\text { standards in the areas of optics and optical measurements } \\
\text { - Co-Chair of the Optical Society of America (OSA) Topical Meeting on Optical Fabrica- } \\
\text { tion and Testing, October 2008, Rochester, NY } \\
\text { - Program Committee member of the Optical Society of America (OSA) Topical Meeting } \\
\text { on Optical Fabrication and Testing }\end{array}$ \\
\hline Ivester, Rob & $\begin{array}{l}\text { - Chair of the 11th CIRP Conference on Modeling of Machining Operations, Gaithers- } \\
\text { burg, MD [2008] } \\
\text { - Completed the one-year NIST New Leaders Program training for leadership and man- } \\
\text { agement skills [2008] } \\
\text { - NIST Program Analyst, NIST Director's Office [2008-2010] }\end{array}$ \\
\hline Jabbour, Zeina & $\begin{array}{l}\text { - Invited Member and Member of Steering Committee, CIPM Consultative Committee } \\
\text { on Mass and Related Quantities (CCM) working group on redefinition of the SI units } \\
\text { - Member of Steering Committee, CIPM Consultative Committee on Mass and Related } \\
\text { Quantities (CCM) working group on uncertainty components in mass metrology } \\
\text { - Member, International Organizing Committee for the } 2010 \text { IMEKO Technical Commit- } \\
\text { tee } 3 \text { on "Measurement of Force, Mass, and Torque," November, 2010, Thailand }\end{array}$ \\
\hline Lee, Kang & $\begin{array}{l}\text { - Chair, IEEE Instrumentation and Measurement Society TC9 Sensor Technology } \\
\text { Committee } \\
\text { - Chair, Sensor Standards Harmonization Working Group for the Department of Home- } \\
\text { land Security } \\
\text { - Member, International Committee for Information Technology Standards (INCITS) Ad } \\
\text { Hoc working group on Sensor Networks } \\
\text { - Technical Co-chair, International Symposium on Precision Clock Synchronization } \\
\text { (ISPCS), September 22-26, 2008, University of Michigan } \\
\text { - General Co-chair, International Symposium on Precision Clock Synchronization } \\
\text { (ISPCS), October 12-16, 2009, University of Brescia, Italy } \\
\text { - Session Chair, Performance of Precision Synchronization Systems, International Sym- } \\
\text { posium on Precision Clock Synchronization (ISPCS), October 12-16, 2009, University } \\
\text { of Brescia, Italy. } \\
\text { - Workshop Chair, Wireless Sensors for Shop Floor Monitoring, October 6, 2009, ISA } \\
\text { Expo, Houston, TX }\end{array}$ \\
\hline
\end{tabular}




\begin{tabular}{|c|c|}
\hline Staff & Leadership \\
\hline Lee, Vincent & $\begin{array}{l}\text { - Completed the year-long NIST "Building The Next Generation" leadership training } \\
\text { program [2008] }\end{array}$ \\
\hline Moylan, Shawn & $\begin{array}{l}\text { - Member, Organizing Committee for SME MicroManufacturing Conference [2008, } \\
\text { - Member, 2010] } \\
\text { Operations [2008] } \\
\text { - Co-Editor, Proceedings of the } 11^{\text {th }} \text { CIRP Conf on Modeling of Machining } \\
\text { Operations [2009] } \\
\text { - Co-Chair, 2010 ASME Symposium on "Advances in Micro-Scale Manufacturing and } \\
\text { Metrology Systems" }\end{array}$ \\
\hline Nedzelnitsky, Victor & $\begin{array}{l}\text { - Technical advisor to the U.S. National Committee of the IEC for the IEC TC29 Stan- } \\
\text { dards Committee "Electroacoustics" } \\
\text { - Chair, ANSI/ASA S1/WG1 Standards Committee "Standard Microphones and their } \\
\text { Calibration" }\end{array}$ \\
\hline Shaw, Gordon & $\begin{array}{l}\text { - Vice-chair, MEMS and Nanotechnology Technical Division for the Society for Experi- } \\
\text { mental Mechanics Annual Meeting [2009 and 2010] } \\
\text { - Co-organizer and Session Chair on Nanotechnology at the } 2009 \text { Society for Experi- } \\
\text { mental Mechanics (SEM) Annual Conference, Albuquerque, NM }\end{array}$ \\
\hline Soons, Johannes & $\begin{array}{l}\text { - Chair, ANSI/ASME B5/TC56 Standards Committee "Information Technology for } \\
\text { Machine Tools" } \\
\text { - Alternate Member, ANSI/ASME B5/TC52 Standards Committee "Machine Tool } \\
\text { Performance" } \\
\text { - Member, ANSI/ASME B5 Standards Committee "Machine Tools - Components, Ele- } \\
\text { ments, Performance, and Equipment" } \\
\text { - Member, ANSI/ASME U.S. Technical Advisory Group to ISO/TC39 - Machine Tools } \\
\text { - Member, ASTM Technical Committee F42 on Additive Manufacturing Technologies }\end{array}$ \\
\hline Wagner, Randy & - Vice-Chair, SAE Emergency Vehicle Siren Task Force \\
\hline Whitenton, Eric & $\begin{array}{l}\text { - Completed the year-long NIST "Building The Next Generation" leadership training } \\
\text { program [2009] }\end{array}$ \\
\hline
\end{tabular}




\section{Excellence}

\begin{tabular}{|c|c|}
\hline Staff & Excellence Recognized \\
\hline Chesnutwood, Kevin & $\begin{array}{l}\text { - Recipient, Best Paper and Overall Best Paper Awards at the } 2009 \text { National Conference } \\
\text { on Standards Laboratories International (NCSL-I) Annual Symposium for the paper } \\
\text { "Automating Data Acquisition on a Mechanical Equal Arm Balance Used in Large Mass } \\
\text { Calibrations" } \\
\text { - Participant in } 2008 \text { BIPM Metrology Summer Program }\end{array}$ \\
\hline Chu, Jiyoung & $\begin{array}{l}\text { - Invited Speaker, "Measuring the Phase Transfer Function of a Phase-Shifting Interferom- } \\
\text { eter," SPIE Optics and Photonics Conference, San Diego, CA, August } 2008\end{array}$ \\
\hline Cooke, April & $\begin{array}{l}\text { - Best Poster Runner-up in the Engineering Category during the annual NIST Sigma XI } \\
\text { postdoctoral poster presentation, for the poster "High-Bandwidth Imaging of Serrated } \\
\text { Chip Formation During Orthogonal Cutting of Ti-6AL-4V," [2009] } \\
\text { - Profiled in the Winter 2009/Spring } 2010 \text { Minority College Issue of Diversity/Careers in } \\
\text { Engineering and Information Technology, a magazine and web site focused on technical } \\
\text { careers for groups traditionally underrepresented in technology }\end{array}$ \\
\hline Donmez, Alkan & $\begin{array}{l}\text { - Guest Editor, International Journal of Mechatronics and Manufacturing Systems (IJMMS), } \\
\text { Special Issue on "Smart Machining Systems" [Vol. 2, No.5/6, 2009] } \\
\text { - Invited Speaker, "New Standards on Machine Tool Performance Testing," Machine } \\
\text { Metrology for the 21st Century, Clemson University International Center for Automotive } \\
\text { Research [2010] } \\
\text { - Invited Speaker, "Axis of Rotation Metrology Applied to Machine Tool Rotary Tables," } \\
\text { International Machine Tool Engineers Conference, Taichun, Taiwan [2008] } \\
\text { - Invited Speaker, "National and International Machine Tool Performance Standards," } \\
\text { ASPE Special Seminar at the International Manufacturing Technology Show [2008] } \\
\text { - Invited Speaker, "A Novel Cooling System to Reduce Thermally-Induced Errors of } \\
\text { Machine Tools," Special CIRP Seminar at the Swiss Federal Institute of Technology } \\
\text { (ETH), January 22, 2008, Zurich, Switzerland }\end{array}$ \\
\hline Grie & $\begin{array}{l}\text { - Invited Speaker, seminar on "New Trends in Flatness Measurement Technology," Physi- } \\
\text { kalisch-Technische Bundesanstalt (PTB), Braunschweig, Germany, October 20, } 2009 \\
\text { - Invited Speaker, "New Method for Deformation-Free Flatness Measurements of Thin } \\
\text { Optics and EUV Photomask Blanks," Max Planck Institute for the Physics of Light (MPL), } \\
\text { Erlangen, Germany, October 22, } 2009 \\
\text { - Invited visit to the Australian Centre for Precision Optics (ACPO), part of the Australian } \\
\text { national science and research organization CSIRO, Australia, July 6-20, 2009. Visit co- } \\
\text { sponsored by CSIRO. }\end{array}$ \\
\hline Ivester, Rob & $\begin{array}{l}\text { - Recipient, } 2008 \text { NIST Bronze Medal Award for Superior Federal Service for outstanding } \\
\text { technical achievements that have dramatically advanced the state-of-the-art of measure- } \\
\text { ment science for manufacturing processes }\end{array}$ \\
\hline Jabbour, Zeina & $\begin{array}{l}\text { - Invited Speaker, "Redefinition of the Kilogram: Status and Impact on Mass Metrology," } \\
\text { Measurement Science Conference, March 13-14, 2008, Anaheim, CA } \\
\text { - Featured in an episode of the Modern Marvels television show on the topic of "Mea- } \\
\text { surement," History Channel, } 2008\end{array}$ \\
\hline
\end{tabular}




\begin{tabular}{|c|c|}
\hline Staff & Excellence Recognized \\
\hline Lee, Kang & $\begin{array}{l}\text { - Recipient, two Certificates of Appreciation from the IEEE Standards Board for contribu- } \\
\text { tions to development of the IEEE } 1451.0 \text { and } 1451.5 \text { standards [2009] } \\
\text { - Keynote Speaker, "Smart Sensor Standards for Interoperable Sensor Networks," 9th } \\
\text { International Conference on Electronic Measurement \& Instruments (ICEMI 2009), } \\
\text { August 16-18, 2009, Beijing, China } \\
\text { - Invited expert on smart sensor networks and standards, Austrian Academy of Sciences, } \\
\text { Research Unit for Integrated Sensor Systems } \\
\text { - Invited Speaker, "IEEE 1451.5 wireless sensors," NASA Space Sensors and Measurement } \\
\text { Techniques Workshop, August 5-6, 2008, Newport News, VA } \\
\text { - Invited Speaker, "Sensors Standards," DoD Unattended Ground Sensor Standards Work- } \\
\text { ing Group, June 23, 2009, VA } \\
\text { - Invited Speaker "Global Sensors Standards Harmonization," U.S. Measurement and } \\
\text { Signature Intelligence (MASINT) workshop, August 12, 2009 } \\
\text { - Invited Speaker, "Smart Sensors and Sensor Networks," Institute of Instrument Science, } \\
\text { Tsinghua University, August 19, 2009, Beijing, China }\end{array}$ \\
\hline Pratt, Jon & $\begin{array}{l}\text { - Recipient, } 2008 \text { Nano } 50 \text { Award for "Contributions to the field of nanomechanical test } \\
\text { instrumentation and as a pioneer in the development of standards and practices that } \\
\text { provide common, traceable references that enable instrument manufacturers and users } \\
\text { to compare across measurement platforms and among competing methodologies" } \\
\text { - Invited Speaker, "Mechanical Metrology at the Scale Where Top-Down Meets Bottom- } \\
\text { Up Manufacturing," BIPM Workshop on Metrology at the Nano-Scale, Sevres, France, } \\
\text { February, } 2010\end{array}$ \\
\hline Seifarth, Rick & - Participant in 2008 BIPM Metrology Summer Program \\
\hline Shaw, Gordon & $\begin{array}{l}\text { - Recipient, } 2008 \text { Nano } 50 \text { Award for "Contributions to the field of nanomechanical test } \\
\text { instrumentation and as a pioneer in the development of standards and practices that } \\
\text { provide common, traceable references that enable instrument manufacturers and users } \\
\text { to compare across measurement platforms and among competing methodologies" }\end{array}$ \\
\hline Soons, Johannes & $\begin{array}{l}\text { - Recipient, Certificate of Appreciation from the ASME Council on Codes and Standards } \\
\text { for service as Chair of the B5 Technical Committee } 56 \text { "Information Technology for } \\
\text { Machine Tools" [2009] } \\
\text { - Recipient, Certificate of Recognition from the ASME Council on Codes and Standards } \\
\text { for "Dedicated Voluntary Service to ASME Codes and Standards" [2009] }\end{array}$ \\
\hline Whitenton, Eric & $\begin{array}{l}\text { - Recipient, } 2008 \text { NIST Bronze Medal Award for Superior Federal Service for outstanding } \\
\text { technical achievements that have dramatically advanced the state-of-the-art of measure- } \\
\text { ment science for manufacturing processes } \\
\text { - Recipient, } 2009 \text { NAMRI/SME Outstanding Paper Award for the paper "High-Speed } \\
\text { Microvideography Observations of the Periodic Catastrophic Shear Event in Cutting AISI } \\
1045 \text { Steel," co-authored with Jarred Heigel }\end{array}$ \\
\hline
\end{tabular}




\section{ISD Staff Recognition \\ Other evidence of technical excellence, not necessarily tied to above pro- grams, but supporting Division Projects and Mission}

- Chair, ASTM E54.08.01 Homeland Security Applications, Operational Equipment, Urban
Search \& Rescue Robots (2010)
- Executive Committee, RoboCup Rescue Robot League, 2006-2010
- Member, U. S. RoboCup Committee
- Chair, RoboCup Rescue Robot League, which has grown under his leadership to be one of
the premier international competitions stimulating advancements in mobile robot autonomy
and mobility
- Award for Excellence in Physical Sciences and Mathematics for Springer Handbook of
Robotics, 2008
- Certificate of Appreciation from the Montgomery County Fire and Rescue Service for host-
ing several national capital region bomb squads in a three-day field training class in impro-
vised explosive device disposal (IEDD) in preparation for their deployment to the National
Mall during the 2009 Presidential inauguration.
- Certificate of Recognition and Appreciation, "for continued support and assistance provided
to the National Capital Region Bomb Squad Community for conducting full scale robotic
testing and evaluation for selection and procurement." 2009
- Special Act Award from the Department of the Army, for "significant contributions in the
initiation of a major new Collaborative Technology Alliance in Robotics." 2010




\begin{tabular}{|c|c|}
\hline R & 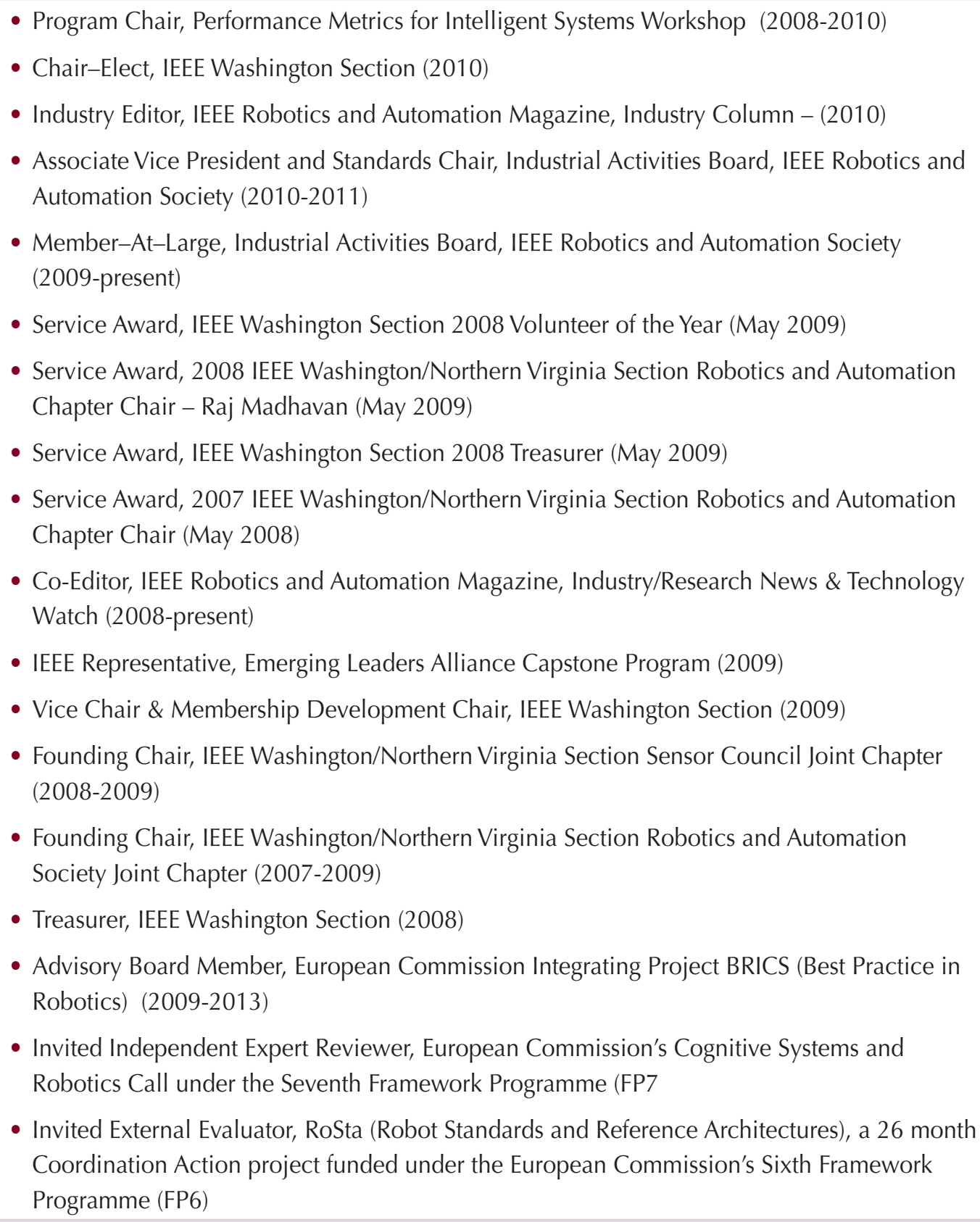 \\
\hline $\begin{array}{l}\text { Craig Schlenoff, } \\
\text { Michelle Steves } \\
\text { (ITL), Ann Virts, } \\
\text { Brian Weiss }\end{array}$ & $\begin{array}{l}\text { - Department of Commerce Bronze Medal "For developing and administering a } \\
\text { groundbreaking performance evaluation framework for characterizing complex } \\
\text { intelligent systems." (2008) }\end{array}$ \\
\hline $\begin{array}{l}\text { Craig Schlenoff, } \\
\text { Brian Weiss }\end{array}$ & $\begin{array}{l}\text { - Track Chairs, "Test Methods and Performance Evaluations for Intelligent Systems", Interna- } \\
\text { tional Test and Evaluation Association (ITEA) Annual Conference (2009) }\end{array}$ \\
\hline Brian Weiss & - Best Paper and Best Presentation Awards ITEA annual Conference (2009) \\
\hline Zeid Kootbally & - Best Presentation Award, ITEA annual Conference (2009) \\
\hline Hui-Min Huang & $\begin{array}{l}\text { - Chair, SAE AS4-D Committee on Unmanned Systems Performance Measures Committee } \\
\text { - Task Group Chair, Terminology effort in ASTM E54.08.01 Response Robotics } \\
\text { - Chair, Autonomy Levels for Unmanned Systems (ALFUS) Working Group }\end{array}$ \\
\hline
\end{tabular}




\begin{tabular}{|c|c|}
\hline $\begin{array}{l}\text { Raj Madhavan, } \\
\text { Elena Messina }\end{array}$ & $\begin{array}{l}\text { - Co-Founders and Co-Chairs, IEEE-RAS Technical Committee, "Performance Evaluation and } \\
\text { Benchmarking Robotic and Automation Systems" (TC-PEBRAS) (2009-present) } \\
\text { - Editors (with Eddie Tunstel of JHU Applied Physics Laboratory), Performance Evaluation and } \\
\text { Benchmarking of Intelligent Systems, Springer Books, 2009. }\end{array}$ \\
\hline Elena Messina & $\begin{array}{l}\text { - Chair, ASTM E54.08.01 Homeland Security Applications, Operational Equipment, Urban } \\
\text { Search \& Rescue Robots (2008-2009) } \\
\text { - Advisory Board Member, Texas A\&M Texas Engineering Extension Service } \\
\text { - Member of Research Management Board for Army Research Laboratory's Micro Autono- } \\
\text { mous Science and Technology Collaborative Technology Alliance } \\
\text { - General Chair, Performance Metrics for Intelligent Systems 2008, 2009, } 2010 \\
\text { - Invited Distinguished Lecture, Case Western Reserve University, } 2009 \\
\text { - Invited Lecture on Homeland Security Technologies, University of South Florida, } 2008 \\
\text { - Invited Speaker, Mid-Atlantic Technology Showcase, } 2008 \\
\text { - Workshops Chair, American Nuclear Society Joint Topical Conference, } 2008 \\
\text { - Organizer/Co-chair: ANS Robots for Emergency Response Workshop, part of Joint Topical } \\
\text { Conference Emergency Preparedness and Robotics for Hazardous Environments } \\
\text { (March 2008) } \\
\text { - Keynote Speaker, IEEE International Conference on Intelligent Robots and Systems Work- } \\
\text { shop on "Performance Evaluation and Benchmarking for Next Intelligent Robots and } \\
\text { Systems" 2009 } \\
\text { - Participant in NSF/CCC/CRA Robotics Roadmapping Workshop (Manufacturing and Auto- } \\
\text { mation Systems) } \\
\text { - Special Act Award from the Department of the Army, for " significant contributions in the } \\
\text { initiation of a major new Collaborative Technology Alliance in Robotics." } 2010\end{array}$ \\
\hline $\begin{array}{l}\text { Sandor Szabo, } \\
\text { Joe Falco, Rick } \\
\text { Norcross }\end{array}$ & $\begin{array}{l}\text { - Department of Commerce Bronze Medal Award - For the development of performance test } \\
\text { and measurement methods for automotive crash warning systems, } 2009 \\
\text { - NHTSA - Plaque - "In recognition of dedication and technical contributions to the success- } \\
\text { ful execution of the Intelligent Vehicle-Based Safety Systems (IVBSS) Verification Tests" } 2009\end{array}$ \\
\hline
\end{tabular}




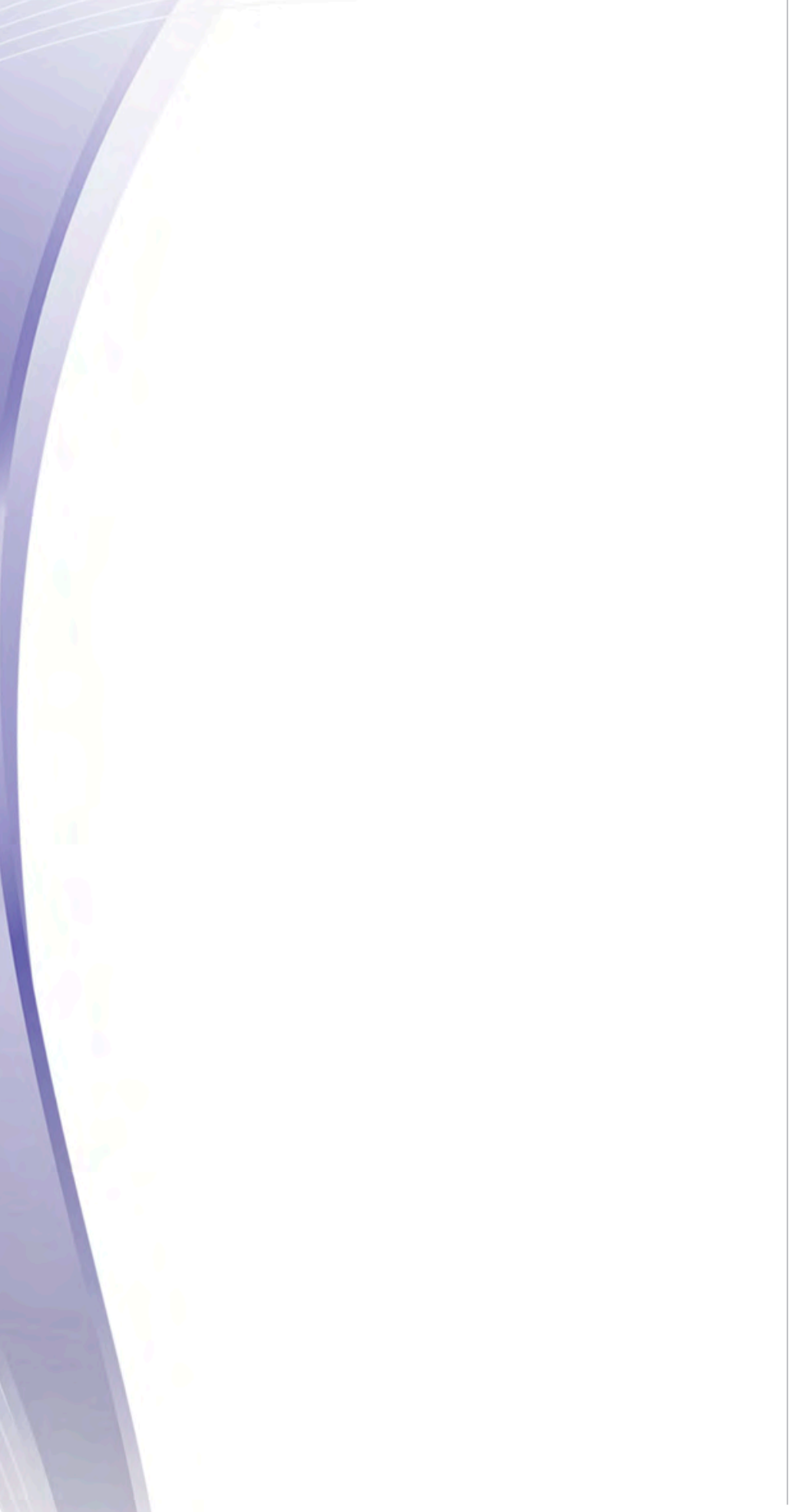

148 


\section{Publications}




\section{Precision Engineering Division}

List of Publications

For

FY2008 to FY2010

Publication Type - All Publication Type

(October 1, 2007 to March 1, 2010) 


\section{FY2010 Publications:}

(R) D. E. Gilsinn, B. R. Borchardt, A. Tebbe, "Volume Estimation of Molded Artifacts by B-Splines" Proceedings of the SIAM Conference on Mathematics for Industry: Challenges and Frontiers, San Francisco, CA, United States, 10/09/2009 to 10/10/2009, 0 pp., (09-Oct-2009)

(I) R. Eastman, T. H. Hong, J. Shi, T. Hanning, B. Muralikrishnan, S. S. Young, T. Chang, "Performance Evaluation and Metrics for Perception in Intelligent Manufacturing" Performance Evaluation and Benchmarking of Intelligent Systems, Chapter: -, Springer, 101 Philip Drive, Norwell, MA, 02061, United States, pp. 269-310, (31-Dec-2009)

\section{FY2009 Publications:}

(R) S. Smith, A. Tsiamis, M. McCallum, A. Hourd, J. Stevenson, A. Walton, R. G. Dixson, R. A. Allen, J. E. Potzick, M. W. Cresswell, N. Orji, "Comparison of Measurement Techniques for Linewidth Metrology on Advanced Photomasks" IEEE Transactions on Semiconductor Manufacturing, Vol. 22, pp. 72-79, (19-Feb-2009)

M. Coll Bau, C. Hacker, L. H. Miller, D. R. Hines, E.C. Williams, C. A. Richter, "Ultrasmooth Gold as a Top Metal Electrode for Molecular Electronic Devices" Electrochemical Society Transactions, Vol. 16, No. 25, pp. 139-146, (07-Apr-2009)

J. J. Gorman, Y.S. Kim, A. E. Vladar, N. G. Dagalakis, "Design of an on-chip microscale nanoassembly system" International Journal of Nanotechnology, Vol. 6, pp. 710-721, (10-Feb-2009)

(R) J. A. Stone, J. Decker, P. Gill, A. Lewis, P. Juncar, D. Rovera, M. Villiseid, "Advice from the CCL on the use of unstabilized lasers as standards of wavelength: the helium-neon laser at 633 nm" Metrologia, Vol. 46, pp. 11-18, (01-Jan-2009)

M. T. Postek, A. E. Vladar, "Helium Ion Microscopy and its Application to Nanotechnology and Nanometrology "Scanning, Vol. 30, pp. 457-462, (04-Nov-2008)

J. Li, Y.L. Shen, J. Jeong, F. Scire, J. A. Kramar, "A COMPACT, COMPOUND ACTUATOR FOR THE MOLECULAR MEASURING MACHINE" American Society for Precision Engineering, Annual Meeting | 23rd | Proceedings of ASPE 23rd Annual Meeting | American Society for Precision Engineering, American Society for Precision Engineering, Annual Meeting | 23rd | Proceedings of ASPE 23rd Annual Meeting | American Society for Precision Engineering, Portland, OR, United States, 10/19/2008 to 10/24/2008, 4 pp., (19-Oct-2008)

(R) S. C. Feng, C. B. Joung, T. V. Vorburger, "Characterization of Probe Dynamic Behaviors in Critical Dimension Atomic Force Microscopy" International Journal of Nanotechnology, (15-Oct-2008)

T. A. Germer, H. Patrick, R. M. Silver, B. Bunday, "Developing an Uncertainty Analysis for Optical Scatterometry" Metrology, Inspection, and Process Control for Microlithography XXIII, San Jose, CA, United States, 02/22/2009 to 02/27/2009, (03-Aug-2009)

Z. H. Levine, S. E. Grantham, D. S. Sawyer, A. P. Reeves, D. F. Yankelevitz, "A Low Cost Fiducial Reference for Computed Tomography" Academic Radiology, Vol. 113, No. 6, pp. 335-340, (11-Nov-2008)

\footnotetext{
Legend:

F - NIST 114 Filed

S - Submitted to ERB

A - ERB Approved

R - Refereed

I - Invited
} 
(R) G. Stan, C. Ciobanu, T. Thayer, G. Wang, R. Creighton, P. P. Kavuri, L. A. Bendersky, R. F. Cook, "Elastic Modulus of Faceted Aluminum Nitride Nanotubes Measured by Contact Resonance Atomic Force Microscopy" Nanotechnology, Vol. 20, No. 3, 4 pp., (17-Dec-2008)

(R) L. Pakstis, A. Zheng, T. V. Vorburger, J. Dunkers, T. P. Quinn, M. T. Cicerone, "Evaluation of Polydimethylsiloxane Modification Methods for Cell Response" Journal of Biomedical Materials Research Part A, (20-Feb-2009)

K. W. Lyons, M. T. Postek, "A Decade of Commitment from the NIST Manufacturing Engineering Laboratory to Nanomanufacturing and Nanometrology" Proceedings of the SPIE Optics and Photonics NanoScience and Engineering Conference 2009, San Diego, CA, United States, 08/02/2009 to 08/06/2009, (27-Aug-2009)

C. W. Jones, J. A. Kramar, S. Davidson, R. Leach, J. R. Pratt, "Comparison of NIST SI Force Scale to NPL SI Mass Scale" Proceedings from the American Society for Precision Engineering, 2008, American Society for Precision Engineering 23rd Annual Meeting, Portland, OR, United States, 10/19/2008 to 10/24/2008, 4 pp., (19-Oct-2008)

J. S. Villarrubia, Z. Ding, "Sensitivity of SEM width measurements to model assumptions" Proceedings of SPIE Advanced Lithography, Vol 7272, San Jose, CA, United States, 02/23/2009 to 02/27/2009, (31-Mar-2009)

(R) F. Tian, X. Qian, J. S. Villarrubia, "Blind estimation of general tip shape in AFM imaging" Ultramicroscopy, Vol. 109, pp. 4453, (01-Dec-2008)

D. E. Gilsinn, B. R. Borchardt, A. Tebbe, "Estimating Volumes of Simulated Lung Cancer Nodules" NIST Interagency/Internal Report (NISTIR) 7571, (15-Jul-2009)

\section{FY2008 Publications:}

J. A. Stone, "Using uncalibrated lasers as wavelength standards" Proceedings, Simposio de Metrología 2008, Queretaro, -1, Mexico, 10/22/2008 to 10/24/2008, 1 pp., (01-Oct-2008)

(R) M. W. Cresswell, R. A. Allen, W. F. Guthrie, C. E. Murabito, R. G. Dixson, A. Hunt, "Comparison of SEM and HRTEM CD Measurements Extracted from Test-Structures Having Feature Linewidths from $40 \mathrm{~nm}$ to $240 \mathrm{~nm}$ "IEEE Transactions on Instrumentation and Measurement, Vol. 57, No. 1, pp. 100-109, (01-Jan-2008)

(R) J.F. Song, P. Rubert, X. A. Zheng, T. V. Vorburger, "Topography Measurements for Determining the Decay Factors in Surface Replication" Measurement Science \& Technology, Vol. 19, No. 8, pp. 1-5, (04-Jul-2008)

(R) B. Muralikrishnan, J. A. Stone, J. R. Stoup, "Area Measurement of Knife Edge and Cylindrical Apertures using Ultra Low Force Contact Fiber Probe on a CMM" Metrologia, Vol. 45, No. 3, pp. 281-289, (01-Jan-2008)

(R) D.I. Kim, J. Grobelny, P. Namboodiri, R. F. Cook, "Origin of Adhesion in Humid Air" Langmuir, Vol. 24, pp. 1873-1877, (18Apr-2008)

(R) K.H. Chung, S. Scholz, G. A. Shaw, J. A. Kramar, J. R. Pratt, "SI traceable calibration of an instrumented indentation sensor spring constant using electrostatic force" Review of Scientific Instruments, Vol. 79, No. 9, pp. 095105-095105-8, (22-Sep-

\footnotetext{
Legend:

F - NIST 114 Filed

S - Submitted to ERB

A - ERB Approved

R - Refereed

I - Invited
} 
2008)

T. V. Vorburger, R. G. Dixson, J. N. Fu, N. Orji, S. C. Feng, M. W. Cresswell, R. A. Allen, W. F. Guthrie, W. Chu, "Nano- and Atomic-Scale Length Metrology" Proc. 5th Int. Conf. on Precision, Meso, Micro, and Nano Engineering (COPEN 2007) (Allied Publishers Pvt. Ltd, Chennai, 2007), 5th Int. Conf. on Precision, Meso, Micro, and Nano Engineering (COPEN 2007), Trivandrum, India, 12/14/2007 to 12/14/2007, pp. 13-18, (14-Dec-2007)

K. W. Lyons, M. T. Postek, "Metrology at the Nanoscale: What are the Grand Challenges?" SPIE NanoScience and Engineering, San Diego, CA, United States, 08/10/2008 to 08/14/2008, 12 pp., (06-Aug-2008)

M. T. Postek, A. E. Vladar, J. A. Dagata, N. Farkas, B. Ming, R. Sabo, T. H. Wegner, "Cellulose Nanocrystals the Next Big Nano-thing?" Proceedings of SPIE, Instrumentation, Metrology, and Standards for Nanomanufacturing, San Diego, CA, United States, 08/10/2008 to 08/14/2008, Vol. 7042, (06-Aug-2008)

(R) R. Attota, R. M. Silver, R. G. Dixson, "Line Width Measurement Technique Using Through-Focus Optical Images" Applied Optics, Vol. 47, pp. 495-503, (01-Jan-2008)

N. G. Dagalakis, Y.S. Kim, D. S. Sawyer, C. M. Shakarji, "Development of Tools for Measuring the Performance of Computer Assisted Orthopaedic Hip Surgery Systems" Proceedings of the Performance Metrics for Intelligent Systems | 2007 | PerMIS |, Performance Metrics for Intelligent Systems Workshop (PerMIS) 2007, Gaithersburg, MD, 08/29/2007 to 08/30/2007, (28-Dec2007)

N. G. Dagalakis, J. B. Stiehl, Y.S. Kim, D. S. Sawyer, C. M. Shakarji, "NIST Medical Phantom Device to Assist With the Calibration and Performance Testing of CAOS Systems" NIST Medical Phantom Device to Assist With the Calibration and Performance Testing of CAOS Systems, , (09-Nov-2007)

(R) J. Clarke, M. Schmidt, N. Orji, "Photoresist Cross-sectioning with Negligible Damage using a Dual-beam FIB-SEM: A High Throughput Method for Profile Imaging" Journal of Vacuum Science and Technology B, Vol. 25, No. 6, pp. 2526-2530, (01-Nov-2007)

(R) H. Patrick, R. Attota, B. M. Barnes, T. A. Germer, M. T. Stocker, R. M. Silver, M. R. Bishop, "Optical Critical Dimension Measurement of Silicon Grating Targets Using Back Focal Plane Scatterfield Microscopy" Journal of Microlithography Microfabrication and Microsystems, Vol. 7, No. 1, pp. 013012-1-013012-11, (02-Jan-2008)

D.I. Kim, P. Namboodiri, F. DelRio, R. F. Cook, "Mechanical and electrical coupling at metal-insulator-metal nano-scale contacts" Applied Physics Letters, Vol. 93, (22-May-2008)

C. Wang, R. L. Jones, K.W. Choi, C. Soles, E. K. Lin, W.L. Wu, J. S. Clarke, J. S. Villarrubia, B. Bunday, "Line Width Roughness and Cross Sectional Measurements of Sub-50 nm Structures with CD-SAXS and CD-SEM" SPIE Conference Proceedings, Vol. 6922, 6 pp., (24-Mar-2008)

(R) T. D. Doiron, "Gauge Blocks - A Zombie Technology" Journal of Research (NIST JRES), Vol. 113, No. 3, pp. 175-184, (01May-2008)

\footnotetext{
Legend:

F - NIST 114 Filed

S - Submitted to ERB

A - ERB Approved

R - Refereed

I - Invited
} 
S. C. Feng, C. B. Joung, T. V. Vorburger, "A Characterization of Probe Dynamic Behaviors in Critical Dimension Atomic Force Microscopy" Scanning 2008, Gaithersburg, MD, 04/15/2008 to 04/17/2008, 2 pp., (17-Mar-2008)

M. T. Postek, A. E. Vladar, "The Potentials of Helium Ion Microscopy for Semiconductor Process Metrology" Proceedings of SPIE, Proceedings of SPIE--the International Society for Optical Engineering, San Jose, CA, United States, 02/24/2008 to 02/28/2008, Vol. 6922, (06-Feb-2008)

(R) R. N. Kacker, A. Forbes, R. Kessel, K. D. Sommer, "Bayesian Posterior Predictive p-value of Statistical Consistency in Interlaboratory Evaluations "Metrologia, Vol. 45, pp. 512-523, (17-Sep-2008)

(R) R. N. Kacker, A. Forbes, R. Kessel, K. D. Sommer, "Classical and Bayesian Interpretation of the Birge Test of Consistency and Its Generalized Version in Interlaboratory Evaluations" Metrologia, Vol. 45, No. 3, pp. 257-264, (10-Apr-2008)

S. Smith, M. McCallum, A. Hourd, J. T. Stevenson, A. J. Walton, R. G. Dixson, R. A. Allen, J. E. Potzick, M. W. Cresswell, N. Orji, "Comparison of Measurement Techniques for Advanced Photomask Metrology" Proceedings of the 2008 IEEE Conference on Microelectronics Test Structures, 2008 IEEE Conference on Microelectronics Test Structures, Edinburgh, -1, United Kingdom, 03/24/2008 to 03/27/2008, pp. 35-39, (24-Mar-2008)

\section{Legend:}

F - NIST 114 Filed

S - Submitted to ERB

A - ERB Approved

$\mathbf{R}$ - Refereed

I - Invited 


\section{Manufacturing Metrology Division}

List of Publications

For

FY2008 to FY2010

Publication Type - All Publication Type

(October 1, 2007 to March 1, 2010) 
FY2010 Publications:

(R) D. R. Defibaugh, P. J. Abbott, J. A. Fedchak, "Interlaboratory comparison of Helium Low Gas Flow Measurements in the range 10-13 to $10-11 \mathrm{~mol} / \mathrm{s}$ (10-9 to $10-7 \mathrm{~cm} 3 / \mathrm{s}) "$ NCSL International Measure, Vol. 5, pp. 58-68, (01-Mar-2010)

(I) Z. J. Jabbour, "Getting Closer to Redefining the Kilogram" Weighing and Measurement + Magazine, Dave E. Mathieu WAM Pumblishing Company, PO Box 2247, Hendersonville, TN, 37077, United States, pp. 24-26, (30-Oct-2009)

J. C. Heigel, E. P. Whitenton, "The Effects of Integration Time and Size-of-Source on the Temperature Measurement of Segmented Chip Formation Using Infrared Thermography" Proceedings of the ASME 2009 International Manufacturing Science and Engineering Conference, ASME International Manufacturing Science and Engineering Conference, West Lafayette, IN, United States, 10/04/2009 to 10/07/2009, 10 pp., (07-Oct-2009)

(R) K.H. Chung, J. R. Pratt, M. Reitsma, "Lateral Force Calibration: Accurate Procedures for Colloidal Probe Friction Measurements in Atomic Force Microscopy" Langmuir, Vol. 26, No. 2, 3 pp., (19-Jan-2010)

Y.(. Song, K. B. Lee, "An Interoperability Test System for IEEE 1451.5-802.11 Standard

"SAS 2010 - IEEE Sensors and Applications Symposium

, Limerick, -1, Ireland, 02/23/2010 to 02/25/2010, pp. 183-188, (25-Feb-2010)

\title{
FY2009 Publications:
}

(R) T. J. Burns, S. P. Mates, R. Rhorer, E. P. Whitenton, D. Basak, "Dynamic Properties for Modeling and Simulation of Machining: An Update of Results from the NIST Pulse-Heated Kolsky Bar" Proceedings of the 12th CIRP Conference on Modelling of Machining Operations, 12th CIRP Conference on Modelling of Machining Operations, San Sebastian, -1, Spain, 05/07/2009 to 05/08/2009, pp. 255-262, (07-May-2009)

Y.(. Song, K. B. Lee, "Integration of IEEE 1451 Smart Transducers and OGC-SWE Using STWS" SAS 2009 - IEEE Sensors Applications Symposium, New Orleans, LA, United States, 02/17/2009 to 02/19/2009, pp. 298-303, (17-Feb-2009)

Y.(. Song, K. B. Lee, "Service-oriented Sensor Data Interoperability for IEEE 1451 Smart Transducers" the Proceedings of 2009 IEEE International Instrumentation and Measurement Technology Conference, Singapore, -1, Singapore, 05/05/2009 to 05/07/2009, pp. 1043-1048, (05-May-2009)

K. B. Lee, Y.(. Song, "Sensor Alert Web Service for IEEE 1451-Based Sensor Networks" Proceedings of 2009 IEEE International Instrumentation and Measurement Technology Conference, Singapore, -1, Singapore, 05/05/2009 to 05/07/2009, pp. 1049-1054, (05-May-2009)

(R) S. P. Moylan, B. N. Damazo, M. A. Donmez, "Implementation of a metrology frame to improve positioning of micro/mesoscale machine tools" 2009 Transactions of the North American Manufacturing Research Conference, North American Manufacturing Research Conference (NAMRC), Greenville, SC, United States, 05/15/2009 to 05/22/2009, Vol. 37, pp. 573580, (15-Jul-2009)

G. A. Shaw, D. J. Ross, S. E. Fick, W. N. Vreeland, "Contactless Differential Conductivity Detection" Proceedings of

\author{
Legend: \\ F - NIST 114 Filed \\ S - Submitted to ERB \\ A - ERB Approved \\ R - Refereed \\ I - Invited
}


microTAS 2008, microTAS 2008, San Diego, CA, United States, 10/12/2008 to 10/16/2008, 3 pp., (12-Oct-2008)

J. R. Pratt, D. T. Smith, L. Howard, "Accurate Picometers for DC and Low-Frequency Displacement Measurement" ASPE 2009 Spring Topical Meeting Mechanical Metrology and Measurement Uncertainty, ASPE 2009 Spring Topical Meeting Mechanical Metrology and Measurement Uncertainty, Albuquerque, NM, United States, 04/06/2009 to 04/07/2009, 4 pp., (06Apr-2009)

\section{K. B. Lee, S. E. Fick, R. Gao, R. Yan, "IMECE2008-66937}

Local Geometric Projection-Based Noise Reduction for Vibration Signal Analysis in Rolling Bearings" Proceedings of IMECE2008, 2008 ASME International Mechanical Engineering Congress and Exposition, Boston, MA, United States, $10 / 31 / 2008$ to $11 / 06 / 2008$, pp. 1-7, (31-Oct-2008)

C. W. Jones, J. A. Kramar, S. Davidson, R. Leach, J. R. Pratt, "Comparison of NIST SI Force Scale to NPL SI Mass Scale" Proceedings from the American Society for Precision Engineering, 2008, American Society for Precision Engineering 23rd Annual Meeting, Portland, OR, United States, 10/19/2008 to 10/24/2008, 4 pp., (19-Oct-2008)

(R) D. J. Evans, A. Hornikova, S. D. Leigh, A. L. Rukhin, W. E. Strawderman, "Report on Acceleration Comparison SIM.AUV.VK1 - Draft B" Metrologia, Vol. 46, (20-Mar-2009)

(R) S. P. Moylan, D. Hong, B. N. Damazo, J. A. Soons, M. A. Donmez, "Development of a metrology frame to improve the positioning accuracy of micro/meso-scale machine tools" International Journal of Mechatronics and Manufacturing Systems, Vol. 2, No. 5/6, pp. 600-619, (03-Aug-2009)

R. P. Wagner, V. Nedzelnitsky, "Determination of Wave Motion Correction Values Required for Comparison Calibrations of a New Working Standard Microphone." NIST Interagency/Internal Report (NISTIR) 7526, pp. 1-13, (11-Dec-2008)

J. R. Pratt, L. M. Kumanchik, T. L. Schmitz, "A Platform for Observing the Behavior of AFM Cantilevers During Quasi-Static Loading" Proceedings ASPE 2008 Annual Meeting, ASPE 23rd Annual Meeting \& 12th ICPE, Portland, OR, United States, 10/19/2008 to 10/24/2008, 4 pp., (19-Oct-2008)

(R) K. B. Lee, R. X. Gao, R. Yan, L. Zhang, "A Module-Based Software System for Spindle Condition Monitoring" International Journal of Mechatronics and Manufacturing Systems, Vol. 2, No. 5/6, pp. 532-551, (15-Apr-2009)

A. L. Cooke, M. A. Davies, "High-Bandwidth Thermal Microscopy of the Tool-Chip Interface during Orthogonal Machining of AISI 4140 Steel" Proceedings of the 12th CIRP Conference on Modelling of Machining Operations, 12th CIRP Conference on Modelling of Machining Operations, San Sebastian, -1, Spain, 05/07/2009 to 05/08/2009, pp. 389-394, (07-May-2009)

E. P. Whitenton, "Characterization of Uncertainties When Measuring Metal Cutting Temperatures Using Infrared Radiation Thermography" SPIE Defense, Security, and Sensing 2009 Conference - Thermosense XXXI, Orlando, FL, United States, 04/13/2009 to 04/17/2009, Vol. 7299, pp. 72990G-1-72990G-12, (01-Jun-2009)

(R) J. R. Pratt, "SI Traceability: Current status and future trends for forces below 10 micronewtons" Measurement, pp. 1-14, (24Sep-2009)
Legend:
F - NIST 114 Filed
S - Submitted to ERB
A - ERB Approved
R - Refereed
I - Invited 
(R) K.H. Chung, G. A. Shaw, J. R. Pratt, "Accurate Picoscale Forces for Insitu Calibration of AFM" XIX IMEKO World Congress: Fundamental and Applied Metrology, IMEKO XIX World Congress Fundamental, Lisbon, -1, Portugal, 09/06/2009 to 09/11/2009, 10 pp., (03-Sep-2009)

Y.(. Song, K. B. Lee, "A Standards-based Global Ocean Monitoring System" Proceedings for 2009 International Conference on Electronic Measurement \& Instruments (ICEMI)

, Beijing, -1, China, 08/16/2009 to 08/18/2009, pp. 1-445-1-449, (16-Aug-2009)

K. L. Chesnutwood, "Automating Data Acquisition on a Mechanical Equal Arm Balance Used in Large Mass Calibrations" Proceedings of the National Conference of Standards Laboratories International (NCSLI), NCLSI Workshop and Symposium, San Antonio, TX, United States, 07/26/2009 to 07/30/2009, (27-Jul-2009)

S. P. Moylan, G. W. Vogl, "Uncertainty Analysis of a Simple Fringe Projection System" Proceedings of the American Society for Precision Engineering 2009 Summer Meeting, Peoria, IL, United States, 07/07/2009 to 07/08/2009, Vol. 46, pp. 32-35, (01Jul-2009)

G. A. Shaw, K.H. Chung, D. T. Smith, J. R. Pratt, "Methods for transferring the SI unit of force from millinewtons to piconewtons" Proceedings of the Society for Experimental Mechanics Annual Meeting, 2009, Society for Experimental Mechanics Annual Meeting, Albuquerque, NM, United States, 06/01/2009 to 06/05/2009, 5 pp., (01-Jun-2009)

K. B. Lee, Y.(. Song, T. O'Reilly, K. Headley, D. Edgington, C. Rueda, J. Zedlitz, J. Del Rio, E. Delory, S. Fairgrieve, L. Bermudez, "Instrument Interface Standards for Interoperable Ocean Sensor Networks" Proceedings for IEEE OCEANS 2009 Conference, Bremen, -1, Germany, 05/11/2009 to 05/14/2009, pp. 1-9, (11-May-2009)

(I) M. A. Donmez, J. A. Soons, "Impact of automation on precision" Handbook of Automation, Chapter: Chapter 1 - Introduction, Springer, Tiergartenstrasse 17, Heidelberg, 69121, Germany, pp. 117-126, (03-Aug-2009)

(R) G. A. Shaw, J. J. Maurer, S. E. Fick, T. P. Moffat, J. J. Mallett, J. L. Hudson, "Electrochemical Micromachining of Hastelloy B2 with Ultrashort Voltage Pulses" Electrochimica Acta, Vol. 55, No. 3, pp. 952-958, (08-Sep-2009)

(R) K.H. Chung, G. A. Shaw, J. R. Pratt, "Accurate Noncontact Calibration of Colloidal Probe Sensitivities in Atomic Force Microscopy" Review of Scientific Instruments, Vol. 80, No. 6, pp. 065107-1-065107-13, (15-Jun-2009)

(R) G. Gao, J. P. Lehan, W. W. Zhang, U. Griesmann, J. A. Soons, "Computer-Generated Hologram Cavity Interferometry Test for Large X-Ray Mandrels: Design" Optical Engineering, Vol. 46, No. 6, pp. 063602-1-063602-11, (01-Jun-2009)

(R) Z. J. Jabbour, P. J. Abbott, E. R. Williams, R. Liu, V. J. Lee, H.K. Liu, "Linking Air and Vacuum Mass Measurement by Magnetic Levitation" Metrologia, Vol. 46, pp. 339-344, (12-May-2009)

(R) P. J. Abbott, Z. J. Jabbour, R. Liu, E. R. Williams, J. Chalfoun, "A magnetic levitation technique for the simultaneous comparison of mass artifacts in air and vacuum." IEEE Transactions on Instrumentation and Measurement, Vol. 58, No. 4, pp. 878-883, (01-Apr-2009)

\footnotetext{
Legend:

F - NIST 114 Filed

S - Submitted to ERB

A - ERB Approved

R - Refereed

I - Invited
} 
(R) Q. Wang, G. Gao, U. Griesmann, "Radius Measurement of Spherical Surfaces With Large Radii-of-Curvature Using DualFocus Zone Plates" Technical Digest of the 2008 Optical Society of America Optical Fabrication and Testing Meeting, Optical Fabrication and Testing 2008, Rochester, NY, United States, 10/20/2008 to 10/23/2008, 3 pp., (20-Oct-2008)

M. L. McGlauflin, S. P. Moylan, "Error Motions of a Non-orthogonal Rotary Axis" Proceedings ASPE 2008 Annual Meeting, Portland, OR, United States, 10/19/2008 to 10/24/2008, 4 pp., (19-Oct-2008)

\section{FY2008 Publications:}

(R) S. E. Fick, "Long-term Stability of the NIST Standard Ultrasonic Source" Journal of Research of the National Institute of Standards and Technology, Vol. 113, No. 5, pp. 281-286, (01-Oct-2008)

(R) Y.H. Tang, T. W. Bartel, J. E. Sims, "Ratio Calibration of a Digital Voltmeter for Force Measurement Using the Programmable Josephson Voltage Standard" Measure: The Journal of Measurement Science 32813, Vol. 3, No. 2, pp. 70-75, (01-Jun-2008)

(R) J. C. Heigel, R. W. Ivester, E. P. Whitenton, "Cutting Temperature Measurements of Segmented Chips using Dual-Spectrum High-Speed Microvideography" Transactional Analysis Journal 823005, Vol. 36, pp. 73-80, (20-May-2008)

V. J. Lee, Z. J. Jabbour, "Constant Pressure and Vacuum Transporter for $1 \mathrm{~kg}$ Mass Standards" NIST Interagency/Internal Report (NISTIR), No. NISTIR 7515, pp. 1-8, (08-Sep-2008)

Z. J. Jabbour, P. J. Abbott, E. R. Williams, R. Liu, V. J. Lee, "Magnetic Levitation System for the Dissemination of a NonArtifact Based Kilogram" IMEKO International Conference, IMEKO International Conference, Merida, Mexico, 11/27/2007 to 12/01/2007, 11 pp., (27-Nov-2007)

G. W. Vogl, J. J. Gorman, G. A. Shaw, J. R. Pratt, "A New Oscillator for SI-Traceable Measurements in Atomic Force Microscopy" Mechanics Conference to Celebrate the 100th Anniversary of the Department of Engineering Science and Mechanics, Mechanics Conference to Celebrate the 100th Anniversary of the Department of Engineering Science and Mechanics, Blacksburg, VA, 05/29/2008 to 05/30/2008, 1 pp., (29-May-2008)

K. B. Lee, J. T. Cain, "Integrating Sensors and Actuators into RFID Tags" RFID Technology and Applications, Cambridge Press, Boston, MA, , (01-Jan-2008)

P. M. McGuiggan, J. S. Wallace, D. T. Smith, I. Sridhar, Z. W. Zheng, K. L. Johnson, "Contact Mechanics of Layered Elastic Materials: Experiment and Theory" Journal of Physics D-Applied Physics, Vol. 40, No. 19, pp. 5984-5994, (07-Oct-2007)

(I) G. Gao, J. Lehan, U. Griesmann, "Dual-CGH Interferometry Test for X-Ray Mirror Mandrels" Optical Measurement Systems for Industrial Inspection, SPIE Europe Optical Metrology, Muenchen, -1, Germany, 06/15/2009 to 06/18/2009, Vol. 7389, pp. 73891B-1-73891B-5, (15-Jun-2008)

(R) E. P. Whitenton, J. C. Heigel, R. W. Ivester, "Measurement and Characterization of Dynamics in Machining Chip Segmentation" 11th CIRP International Workshop on Modeling of Machining Operations, Gaithersburg, MD, United States, 09/16/2008 to 09/17/2008, pp. 237-246, (16-Sep-2008)

\footnotetext{
Legend:

F - NIST 114 Filed

S - Submitted to ERB

A - ERB Approved

R - Refereed

I - Invited
} 
J. Chu, Q. Wang, J. P. Lehan, U. Griesmann, G. Gao, "Measuring the Phase Transfer Function of a Phase-Shifting Interferometer" Proceedings of SPIE Volume 7064: Interferometry XIV: Applications, SPIE Optics and Photonics 2008, San Diego, CA, United States, 08/10/2008 to 08/14/2008, Vol. 7064, pp. 70640C-1-70640C-8, (13-Aug-2008)

J. C. Heigel, R. W. Ivester, E. P. Whitenton, "Implementation of Dynamic Material Property Data in Finite-Element Modeling Simulations" Proceedings of the 11th CIRP Conference on Modeling of Machining Operations, 11th CIRP Conference on Modeling of Machining Operations, Gaithersburg, MD, United States, 09/16/2008 to 09/18/2008, pp. 113-120, (16-Sep-2008)

(R) Y.(. Song, K. B. Lee, "Sensor Network based on IEEE 1451.0 and IEEE p1451.2-RS232" IIMTC 2008-IEEE International Instrumentation and Measurement Technology Conference , Victoria, Vancouver Island, Canada, 05/12/2008 to 05/15/2008, pp. 1728-1733, (12-May-2008)

Y.(. Song, K. B. Lee, "An Application Framework for IEEE 1588 Standard" Proceedings of 2008 International IEEE Symposium on Precision Clock Synchronization (ISPCS), Ann Arbor, MI, United States, 09/22/2008 to 09/26/2008, pp. 23-28, (29-Sep-2008)

G. W. Vogl, J. J. Gorman, G. A. Shaw, J. R. Pratt, "A New Microdevice for SI-Traceable Forces in Atomic Force Microscopy" SEM XI International Congress \& Exposition on Experimental and Applied Mechanics, Orlando, FL, 06/02/2008 to 06/05/2008, 9 pp., (02-Jun-2008)

J. C. Heigel, S. P. Moylan, R. W. Ivester, "Proceedings of the 11th CIRP Conference on Modeling of Machining Operations" Proceedings of the 11th CIRP Conference on Modeling of Machining Operations, 11th CIRP Conference on Modeling of Machining Operations, Gaithersburg, MD, United States, 09/16/2008 to 09/18/2008, 11 pp., (16-Sep-2008)

(R) Y.(. Song, K. B. Lee, "STWS: A Unified Web Service for IEEE 1451 Smart Transducers" IEEE Transactions on Instrumentation and Measurement, Vol. 57, No. 8, pp. 1749-1756, (01-Aug-2008)

(R) V. Nedzelnitsky, R. P. Wagner, "Non-contact Methods for Measuring Front Cavity Depths of Laboratory Standard Microphones Using a Depth-Measuring Microscope" Journal of Research of the National Institute of Standards and Technology 823015, Vol. 113, No. 2, pp. 97-119, (03-Mar-2008)

(R) K.H. Chung, S. Scholz, G. A. Shaw, J. A. Kramar, J. R. Pratt, "SI traceable calibration of an instrumented indentation sensor spring constant using electrostatic force" Review of Scientific Instruments, Vol. 79, No. 9, pp. 095105-095105-8, (22-Sep2008)

G. W. Vogl, J. J. Gorman, G. A. Shaw, J. R. Pratt, "A New Oscillator for SI-Traceable Measurements in Atomic Force Microscopy" 12th Conference on Nonlinear Vibrations, Dynamics, and Multibody Systems, Blacksburg, VA, 06/01/2008 to 06/05/2008, 2 pp., (01-Jun-2008)

G. A. Shaw, "Reducing Thermal Noise in Molecular Force Spectroscopy" Proceedings of the Society for Experimental Mechanics Annual Meeting

Orlando, FL, 2008, Orlando, FL, 06/02/2008 to 06/05/2008, 6 pp., (02-Jun-2008)

\footnotetext{
Legend:

F - NIST 114 Filed

S - Submitted to ERB

A - ERB Approved

R - Refereed

I - Invited
} 
(R) G. W. Vogl, J. R. Pratt, "Development of a Self-Excited Oscillator for SI-Traceable Measurements in Atomic Force Microscopy" ASME 2008 International Design Engineering Technical Conferences \& Computers and Information in Engineering Conference (IDETC/CIE 2008), New York City, NY, United States, 08/03/2008 to 08/06/2008, pp. 1-10, (03-Aug2008)

U. Griesmann, Q. Wang, N. Laurenchet, J. A. Soons, "Stationary and non-stationary deformations in three-flat tests" Proceedings of the International Commission for Optics 21st meeting, July 7-10, 2008., ICO 212008 Congress, Sydney, Australia, 07/07/2008 to 07/10/2008, pp. 258-258, (07-Jul-2008)

(R) Y.H. Tang, T. W. Bartel, J. E. Sims, "Ratio Calibration of a Digital Voltmeter for Force Measurement Using the Programmable Josephson Voltage Standard" Measure, Vol. 3, No. 2, pp. 70-75, (02-Jun-2008)

(R) K. B. Lee, "Smart and Wireless Sensor Standards for Distributed Measurements" IEEE Transactions on Instrumentation and Measurement, pp. 1-2, (07-Apr-2008)

R. Yan, R. Gao, Z. Li, K. B. Lee, "Modal Parameter Identification from Output-only Measurement Data: Application to Operating Spindle Condition Monitoring" Proceedings of the 8th International Conference on Frontiers of Design and Manufacturing

, ICFDM 2008, Tianjin, China, 09/23/2008 to 09/26/2008, (23-Sep-2008)

Y.(. Song, K. B. Lee, "Understanding IEEE 1451 - Networked Smart Transducer Interface Standard" IEEE Instrumentation and Measurement Magazine 823045, pp. 1-17, (01-Apr-2008)

(R) K.J. Lee, M. A. Donmez, "Repeatability Analysis on the Tool Point Dynamics for Investigations on Uncertainty in Milling Stability" 2007 ASME International Mechanical Engineering Congress \& Exposition (IMECE2007), 2007 ASME International Mechanical Engineering Congress \& Exposition (IMECE2007), Seattle, WA, 11/11/2007 to 11/15/2007 823028, (11-Nov2007)

(R) E. P. Whitenton, R. W. Ivester, J. C. Heigel, "A Novel Peak Detection Algorithm for Use in the Study of Machining Chip Segmentation" ISCA Proceedings of the 20th International Conference on Computers and Their Applications in Industry and Engineering (CAINE-2007), November 7-9, 2007, San Francisco, CA, ISCA 20th International Conference on Computers and Their Applications in Industry and Engineering, San Francisco, CA, 11/07/2007 to 11/09/2007 823018, (07-Nov-2007)

(I) R. Gao, R. Yan, L. Zhang, K. B. Lee, "Condition Monitoring of Operating Spindle Based on Stochastic Subspace Identification" 2007 ASME International Mechanical Engineering Congress and Exposition, 2007 ASME International Mechanical Engineering Congress and Exposition, Seattle, WA, 11/11/2007 to 11/15/2007, pp. 1-7, (11-Nov-2007)

(R) T. W. Bartel, "Statistical Analysis of 4 MN Force Range Key Comparison" Proceedings of the IMEKO 20th TC3, 3rd TC16, and 1st TC22 International Conference, IMEKO 20th TC3, 3rd TC16 \& 1st TC22 International Conference Force, Mass, Torque, Density, Pressure, Vacuum and Vibrations, Merida, Mexico, 11/27/2007 to 12/01/2007 823017, (27-Nov-2007)

Y.(. Song, K. B. Lee, "An Implementation of Smart Transducer Web Services for IEEE 1451-based Sensor Systems" SAS 2007

\footnotetext{
Legend:

F - NIST 114 Filed

S - Submitted to ERB

A - ERB Approved

R - Refereed

I - Invited
} 
March 1, 2010

- IEEE Sensors and Applications Symposium, SAS 2007 - IEEE Sensors and Applications Symposium, San Diego, CA, 02/06/2007 to $02 / 08 / 2007$, pp. 1-6, (06-Feb-2008)

Legend:

F - NIST 114 Filed

S - Submitted to ERB

A - ERB Approved

R - Refereed

I - Invited 


\section{Intelligent Systems Division}

List of Publications

For

FY2008 to FY2010

Publication Type - All Publication Type

(October 1, 2007 to March 1, 2010) 


\section{FY2010 Publications:}

(I) R. Eastman, T. H. Hong, J. Shi, T. Hanning, B. Muralikrishnan, S. S. Young, T. Chang, "Performance Evaluation and Metrics for Perception in Intelligent Manufacturing" Performance Evaluation and Benchmarking of Intelligent Systems, Chapter: -, Springer, 101 Philip Drive, Norwell, MA, 02061, United States, pp. 269-310, (31-Dec-2009)

T. Kramer, F. M. Proctor, "Feature-based Process Planning Based on STEP" Advanced Design and Manufacturing Based on STEP, Springer, Ashbourne House, The Guildway, Old Portsmouth Road, Guildford, Surrey, GU3 1LP, United Kingdom, (31-Dec-2009)

J. A. Horst, "Interoperability and the DMIS experience" Quality Magazine, Maggie McFadden, Managing Editor, 050 IL Route 83, Suite 200, Bensenville, IL, 60106, United States, 5 pp., (30-Oct-2009)

(R) T. Chang, T. H. Hong, M. Shah, R. D. Eastman, "Mathematical Metrology for Evaluating a 6DOF Visual Servoing System" Proceedings of the Performance Metrics for Intelligent Systems (PerMIS) 2009, Gaithersburg, MD, United States, 09/21/2009 to $09 / 23 / 2009,6$ pp., (08-Oct-2009)

J. L. Michaloski, B.E. Lee, F. M. Proctor, S. Venkatesh, S. Ly, "Quantifying the Performance of MT-Connect in a Distributed Manufacturing Environment

" Proceedings of the 2009 ASME International Design Engineering Technical Conferences (DETC), San Diego, CA, United States, 08/30/2009 to 09/02/2009, 7 pp., (08-Oct-2009)

(R) C. I. Schlenoff, "Ontology Formalisms: What is Appropriate for Different Applications?" Proceedings of the Performance Metrics for Intelligent Systems (PerMIS) 2009, Gaithersburg, MD, United States, 09/21/2009 to 09/23/2009, 8 pp., (06-Oct2009)

(I) C. J. Scrapper Jr, R. Madhavan, A. S. Jacoff, R. Lakaemper, A. Censi, A. A. Godil, A. Wagan, "Quantitative Assessment of Robot-generated Maps" Performance Evaluation and Benchmarking of Intelligent Systems, Chapter: -, Springer, 101 Philip Drive , Norwell, MA, 02061, United States, pp. 221-248, (08-Oct-2009)

J. L. Michaloski, T. Kramer, F. M. Proctor, X. Xu, S. Venkatesh, D. Odendahl, "STEPNC++ - An Effective Tool for Featurebased CAM/CNC" Chapter in Advanced Design and Manufacturing Based on STEP", Springer-Verlag, Old Portsmouth Road, Ashbourne House, The Guildway, Guildford, Surrey, GU3 1LP, United Kingdom, 28 pp., (01-Dec-2009)

(RI) S. B. Balakirsky, S. Carpin, G. Dimitoglou, B. Balaguer, "From Simulation to Real Robots with Predictable Results: Methods and Examples" Performance Evaluation and Benchmarking of Intelligent Systems, Chapter: -, Springer, 101 Philip Drive , Norwell, MA, 02061, United States, (01-Dec-2009)

W. P. Shackleford, R. V. Bostelman, "Data Collection Test-bed for the Evaluation of Range Imaging Sensors for ANSI/ITSDF B56.5 Safety Standard for Guided Industrial Vehicles" Proceedings of the Performance Metrics for Intelligent Systems Workshop | 2009 | PerMIS |, Performance Metrics for Intelligent Systems Workshop (PerMIS) 2009, Gaithersburg, MD, United States, 09/21/2009 to 09/23/2009, 6 pp., (29-Oct-2009)

Legend:

F - NIST 114 Filed

S - Submitted to ERB

A - ERB Approved

R - Refereed

I - Invited 
T. H. Hong, E. Messina, H.M. Huang, M. O. Shneier, R. Eastman, J. Shi, J. Wells, "DYNAMIC PERCEPTION WORKSHOP REPORT: Requirements and Standards for Advanced Manufacturing" NIST Interagency/Internal Report (NISTIR) 7664, 34 pp., (14-Jan-2010)

\section{FY2009 Publications:}

W. G. Rippey, "Update on DMIS Certification" CMM Quarterly, (01-Oct-2009)

W. G. Rippey, "The Dimensional Markup Language Specification for Inspection Results Data" CMM Quarterly, (01-Oct-2009) B. A. Weiss, M. Menzel, "Development of Domain-Specific Scenarios for Training and Evaluation of Two-Way, Free Form, Spoken Language Translation Devices" Proceedings of the 2009 International Test and Evaluation Association Symposium, Baltimore, MD, United States, 09/28/2009 to 10/01/2009, (01-Oct-2009)

J. J. Gorman, Y.S. Kim, A. E. Vladar, N. G. Dagalakis, "Design of an on-chip microscale nanoassembly system" International Journal of Nanotechnology, Vol. 6, pp. 710-721, (10-Feb-2009)

(R) R. Madhavan, E. Messina, "Proceedings of the 2008 Performance Metrics for Intelligent Systems (PerMIS 08) Workshop, August 19-21, 2008" Special Publication (NIST SP) 1090, (10-Feb-2009)

(I) E. Messina, "Robots to the Rescue" Crisis Response Journal, Vol. 5, No. 3, pp. 42-43, (30-Jun-2009)

D. Odendahl, S. Venkatesh, J. L. Michaloski, F. M. Proctor, "Standardization of Auxiliary Equipment for Next Generation CNC Machining" ISA 2008 Technical Conference, Houston, TX, United States, 10/14/2008 to 10/17/2008, (17-Oct-2008)

(I) T. Kramer, X. Xu, "STEP in a Nutshell" Advanced Design and Manufacturing Based on STEP, Chapter: STEP in a Nutshell, Springer, 236 Gray's Inn Road, Floor 6, London, -1, WC1X 8HL, United Kingdom, pp. 1-22, (29-Sep-2009)

(I) J. D. Gilsinn, K. Knake, "EtherNet/IP Interoperability Recommendations" Proceedings of the Open DeviceNet Vendor Association (ODVA), 2009 Conference and 13th Annual Meeting, Howey-in-the-Hills, FL, United States, 02/24/2009 to 02/26/2009, 7 pp., (22-Apr-2009)

S. Szabo, J. A. Falco, R. J. Norcross, "An Independent Measurement System for Testing Automotive Crash Warning Systems" NIST Interagency/Internal Report (NISTIR) 7545, 36 pp., (02-Apr-2009)

D. Le, N. G. Dagalakis, "Performance of a Nanopositioner Controller Filter Compensator" Precision Meso/Micro Systems for Nanomanufacturing, (12-Aug-2009)

J. D. Gilsinn, F. Johnson, "Test Tool for Industrial Ethernet Network Performance" Proceedings of the 55th International Instrumentation Symposium, League City, TX, United States, 06/01/2009 to 06/05/2009, (05-Jun-2009)

(I) J. A. Horst, "Reduce Costs and Increase Quality with Information Exchange Standards for Manufacturing Quality" CMM Quarterly - Special DMSC Edition, 12 pp., (04-Sep-2009) 
T. Kramer, J. A. Horst, "System Builders Manual for Version 2.1.5 of the NIST DMIS Test Suite" NIST Interagency/Internal Report (NISTIR) 7610, (19-Aug-2009)

T. H. Hong, R. Eastman, R. V. Bostelman, H.M. Huang, B. McMorris, "Proceedings of the Dynamic Measurement and Control for Autonomous Manufacturing Workshop" NIST Interagency/Internal Report (NISTIR), (29-May-2009)

G. Rosenbush, T. H. Hong, R. D. Eastman, "Super-Resolution Enhancement of Flash LADAR Range Data" NIST Interagency/Internal Report (NISTIR) 7582, (30-Apr-2009)

(I) R. V. Bostelman, J. S. Albus, "Robotic Patient Lift and Transfer" Service Robots, Aleksandar Lazinica, Editor, Kirchengasse 43/3, A-1070, Vienna, -, American Samoa, (31-Dec-2008)

A. S. Jacoff, A. Downs, A.M. Virts, E. Messina, "Stepfield Pallets: Repeatable Terrain for Evaluating Robot Mobility" Proceedings of the 2008 Performance Metrics for Intelligent Systems (PerMIS) Workshop, Gaithersburg, MD, United States, 08/19/2008 to 08/21/2008, pp. 29-34, (31-Dec-2008)

J. A. Horst, "Proprietary Design Information and Fruit Pies" Published in the Collaboration \& Interoperability Market Report 2008, pp. 51 53, (07-Nov-2008)

T. Kramer, J. A. Horst, "Users Manual for Version 2.1.5 of the NIST DMIS Test Suite (for DMIS 5.1)" NIST Interagency/Internal Report (NISTIR) 7603, (19-Aug-2009)

(R) B. A. Weiss, C. I. Schlenoff, "The Impact of Scenario Development on the Performance of Speech Translation Systems Prescribed by the SCORE Framework" Proceedings of the Performance Metrics for Intelligent Systems (PerMIS) 2009, Gaithersburg, MD, United States, 09/21/2009 to 09/23/2009, 8 pp., (25-Sep-2009)

S. B. Balakirsky, R. Madhavan, "Advancing Manufacturing Research Through Competitions" Proceedings of the SPIE Defense Security and Sensing, Orlando, FL, April 13-17, 2009., Orlando, FL, United States, 04/13/2009 to 04/17/2009, (17Apr-2009)

(R) C. I. Schlenoff, B. A. Weiss, M. P. Steves, G. A. Sanders, F. M. Proctor, A.M. Virts, "Evaluating Speech Translation Systems: Applying SCORE to TRANSTAC Technologies" Proceedings of the Performance Metrics for Intelligent Systems (PerMIS) 2009, Gaithersburg, MD, United States, 09/21/2009 to 09/23/2009, 8 pp., (29-Sep-2009)

\section{FY2008 Publications:}

(I) M. O. Shneier, R. V. Bostelman, J. S. Albus, W. P. Shackleford, T. Chang, T. H. Hong, "A Common Operator Control Unit Color Scheme for Mobile Robots" Performance Metrics for Intelligent Systems Workshop | 2007 | PerMIS |, Performance Metrics for Intelligent Systems (PerMIS) 2007, Gaithersburg, MD, 08/28/2007 to 08/30/2007, (28-Dec-2007)

M. O. Shneier, T. Chang, T. H. Hong, W. P. Shackleford, "Learning Traversability Models for Autonomous Mobile Vehicles" Autonomous Robots, Vol. 24, No. 1, pp. 69-86, (21-Nov-2007)

\footnotetext{
Legend:

F - NIST 114 Filed

S - Submitted to ERB

A - ERB Approved

$\mathbf{R}$ - Refereed

I - Invited
} 
R. Madhavan, E. Messina, "Proceedings of the Performance Metrics for Intelligent Systems (PerMIS)2007 Workshop, August 28-30, 2007" Special Publication (NIST SP) 1073, (28-Dec-2007)

C. I. Schlenoff, J. G. Ajot, R. Madhavan, "PRIDE: A Framework for Performance Evaluation of Intelligent Vehicles in Dynamic, On-Road Environments" 2004 Performance Metrics for Intelligent Systems (PerMIS) Workshop, Gaithersburg, MD, 08/24/2004 to 08/26/2004, (24-Jul-2008)

C. J. Scrapper Jr, R. Madhavan, S. B. Balakirsky, "Stable Navigation Solutions for Robots in Complex Environments" Proceedings of the IEEE International Workshop on Safety, Security, and Rescue Robotics (SSRR2007), Rome, -1, Italy, 09/27/2007 to 09/29/2007, (15-Nov-2007)

G. W. Vogl, J. J. Gorman, G. A. Shaw, J. R. Pratt, "A New Oscillator for SI-Traceable Measurements in Atomic Force Microscopy" Mechanics Conference to Celebrate the 100th Anniversary of the Department of Engineering Science and Mechanics, Mechanics Conference to Celebrate the 100th Anniversary of the Department of Engineering Science and Mechanics, Blacksburg, VA, 05/29/2008 to 05/30/2008, 1 pp., (29-May-2008)

R. Madhavan, M. Foedissch, T. Chang, T. H. Hong, "Grouping Sensory Primitives for Object Recognition and Tracking" Applied Imagery Pattern Recognition (AIPR) 2005, Applied Imagery Pattern Recognition (AIPR) 2005, Washington, DC, $10 / 19 / 2005$ to $10 / 21 / 2005$, (03-Dec-2007)

J. S. Albus, "Reverse Engineering the Brain" Proceedings of the Fall Symposium of the American Association for Artificial Intelligence (AAAI), Arlington, VA, United States, 11/07/2008 to 11/09/2008, (09-Nov-2007)

F. M. Proctor, W. G. Rippey, J. A. Horst, J. A. Falco, T. Kramer, "Interoperability Testing for Shop Floor Measurement" Proceedings of the Performance Metrics for Intelligent Systems Workshop | 2007 | PerMIS |, Performance Metrics for Intelligent Systems (PerMIS) 2007, Gaithersburg, MD, 08/28/2007 to 08/30/2007, (28-Dec-2007)

N. G. Dagalakis, J. B. Stiehl, Y.S. Kim, D. S. Sawyer, C. M. Shakarji, "NIST Medical Phantom Device to Assist With the Calibration and Performance Testing of CAOS Systems" NIST Medical Phantom Device to Assist With the Calibration and Performance Testing of CAOS Systems, , (09-Nov-2007)

R. V. Bostelman, J. S. Albus, "Design of the HLPR ChairHome Lift Position and Rehabilitation Chair" NIST Interagency/Internal Report (NISTIR) 7459, (09-Nov-2007)

S. Venkatesh, R. Morihara, J. L. Michaloski, F. M. Proctor, "Closed Loop CNC Manufacturing -- Connecting the CNC to the Enterprise" International Computers and Information in Engineering Conference | 2007 | ASME |, International Computers and Information in Engineering Conference, Las Vegas, NV, United States, 09/04/2007 to 09/07/2007, (26-Nov-2007)

B. A. Weiss, B. Antonishek, R. J. Norcross, "Fused quad audio/visual and tracking data collection to enhance mobile robot and operator performance analyses" SPIE Defense and Security Symposium 2008, SPIE Defense and Security Symposium 2008, Orlando, FL, 03/17/2008 to 03/20/2008, (20-Mar-2008)

C. I. Schlenoff, Z. Kootbally, R. Madhavan, "Driver Aggressivity Analysis Within the Prediction In Dynamic Environments

Legend:

F - NIST 114 Filed

S - Submitted to ERB

A - ERB Approved

R - Refereed

I - Invited 
(PRIDE) Framework" Proceedings of SPIE | 2007 |, SPIE, 04/09/2007 to 04/13/2007, (19-Oct-2007)

R. V. Bostelman, J. S. Albus, "Sensor Experiments to Facilitate Robot Use in Assistive Environments" 1st International Conference on PErvasive Technologies Related to Assistive Environments PETRA 2008, 1st International Conference on PErvasive Technologies Related to Assistive Environments PETRA 2008, Athens, -1, Greece, 07/16/2008 to 07/19/2008, (19Jul-2008)

S. B. Balakirsky, C. J. Scrapper Jr, S. Carpin, "The Evolution of Performance Metrics in the RoboCup Rescue Virtual Robot Competition" Proceedings of the Performance Metrics for Intelligent Systems Workshop | 2007 | PerMIS |, Performance Metrics for Intelligent Systems (PerMIS) 2007, Gaithersburg, MD, 08/28/2007 to 08/30/2007, (28-Dec-2007)

T. Kramer, "Evaluating Manufacturing Machine Control Language Standards: An Implementer s View" Proceedings of PerMIS | 2007 |, PerMIS, 08/28/2007 to 08/30/2007, (28-Dec-2007)

B. A. Weiss, C. I. Schlenoff, "Evolution of the SCORE Framework to Enhance Field-Based Performance Evaluations of Emerging Technologies

" Proceedings of the 2008 Performance Metrics for Intelligent Systems (PerMIS) Workshop, Gaithersburg, MD, United States, 08/19/2008 to 08/21/2008, pp. 1-8, (21-Aug-2008)

J. Wason, W. Gressick, J. Wen, J. J. Gorman, N. G. Dagalakis, "Multi-Probe Assembly" IEEE, (31-Dec-2007)

Z. Kootbally, C. I. Schlenoff, R. Madhavan, S. Foufou, "Fuzzy-Logic-Based Approach for Identifying Objects of Interest in the PRIDE Framework" Proceedings of the 2008 Performance Metrics for Intelligent Systems (PerMIS) Workshop,

Gaithersburg, MD, United States, 08/19/2008 to 08/21/2008, pp. 17-24, (21-Aug-2008)

R. V. Bostelman, J. S. Albus, "HLPR Chair A Novel Patient Transfer Device" Proceedings of the 2008 Performance Metrics for Intelligent Systems (PerMIS) Workshop, Gaithersburg, MD, United States, 08/19/2008 to 08/21/2008, pp. 302-305, (21Aug-2008)

H.M. Huang, E. Messina, T. H. Hong, C. I. Schlenoff, "Ontological Perspectives for Autonomy Performance" Proceedings of the 2008 Performance Metrics for Intelligent Systems (PerMIS) Workshop, Gaithersburg, MD, United States, 08/19/2008 to 08/21/2008, pp. 101-107, (21-Aug-2008)

H.M. Huang, "Autonomy Levels for Unmanned Systems (ALFUS) Framework:Safety and Application Issues" Performance Metrics for Intelligent Systems Workshop | 2007 | PerMIS |, Performance Metrics for Intelligent Systems (PerMIS) 2007, Gaithersburg, MD, 08/28/2007 to 08/30/2007, (28-Dec-2007)

T. H. Hong, S. Hu, S. S. Young, "Performance of Super-Resolution Enhancement for Flash LADAR Data" Proceedings of the 2008 Performance Metrics for Intelligent Systems (PerMIS) Workshop, Gaithersburg, MD, United States, 08/19/2008 to 08/21/2008, pp. 143-148, (21-Aug-2008)

S. Venkatesh, B. Sides, J. L. Michaloski, F. M. Proctor, "Case Study in the Challenges of Integrating CNC Production and Enterprise Systems " 2007 ASME International Mechanical Engineering Congress and Exposition, 2007 ASME International

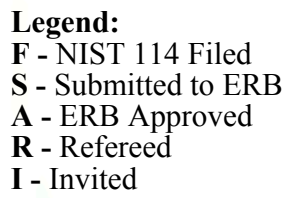


Mechanical Engineering Congress and Exposition, Seattle, WA, 11/11/2007 to 11/15/2007, (03-Dec-2007)

G. W. Vogl, J. J. Gorman, G. A. Shaw, J. R. Pratt, "A New Microdevice for SI-Traceable Forces in Atomic Force Microscopy" SEM XI International Congress \& Exposition on Experimental and Applied Mechanics, Orlando, FL, 06/02/2008 to 06/05/2008, 9 pp., (02-Jun-2008)

G. Roberts, S. B. Balakirsky, S. Foufou, "3D Reconstruction of Rough Terrain for USARSim Using a Height-map Method" Proceedings of the 2008 Performance Metrics for Intelligent Systems (PerMIS) Workshop, Gaithersburg, MD, United States, 08/19/2008 to 08/21/2008, pp. 259-264, (21-Aug-2008)

G. W. Vogl, J. J. Gorman, G. A. Shaw, J. R. Pratt, "A New Oscillator for SI-Traceable Measurements in Atomic Force Microscopy" 12th Conference on Nonlinear Vibrations, Dynamics, and Multibody Systems, Blacksburg, VA, 06/01/2008 to 06/05/2008, 2 pp., (01-Jun-2008)

Z. Kootbally, C. I. Schlenoff, R. Madhavan, "A Brief History of PRIDE" Proceedings of PerMIS | 2007 |, Performance Metrics for Intelligent Systems (PerMIS) 2007, Gaithersburg, MD, 08/28/2007 to 08/30/2007, (28-Dec-2007)

C. T. Pepper, S. B. Balakirsky, C. J. Scrapper Jr, "Robot Simulation Physics Validation" PerMIS Conference | 2007 |, Performance Metrics for Intelligent Systems (PerMIS) 2007, Gaithersburg, MD, 08/28/2007 to 08/30/2007, (28-Dec-2007)

S. B. Balakirsky, F. M. Proctor, C. J. Scrapper Jr, T. Kramer, "A Mobile Robot Control Framework: From Simulation to Reality" International Conference on Simulation, Modeling, and Programming for Autonomous Robots (SIMPAR 2008), Venice, Italy, 11/03/2008 to 11/07/2008, (07-Dec-2007)

S. B. Balakirsky, F. M. Proctor, C. J. Scrapper Jr, T. Kramer, "An Integrated Control and Simulation Environment for Mobile Robot Software Development" Proceedings of IDETC/CIE 2008 ASME 2008 International Design Engineering Technical Conference \& Computers and Information in Engineering Conference, New York, NY, United States, 08/04/2008 to 08/07/2008, (07-Aug-2008)

Z. Kootbally, C. I. Schlenoff, R. Madhavan, S. Foufou, "Performance Evaluation of Cost-Based vs. Fuzzy-Logic-Based Prediction Approaches in PRIDE" SPIE Defense and Security Symposium 2008, Orlando, FL, 03/16/2008 to 03/20/2008, (20Mar-2008)

B. A. Weiss, C. I. Schlenoff, G. A. Sanders, M. P. Steves, S. Condon, J. Phillips, D. Parvaz, "Performance Evaluation of Speech Translation Systems" Proceedings of the 6th edition of the Language Resources and Evaluation Conference, 6th edition of the Language Resources and Evaluation Conference, Marrakech, Morocco, 05/28/2008 to 05/30/2008, (28-May-2008)

(I) J. S. Albus, "A Computational Model of the Human Visual Cortex" Proceedings of the SPIE Defense and Security 2008, SPIE Defense and Security 2008, Orlando, FL, 03/18/2008 to 03/20/2008, (20-Mar-2008)

T. Chang, T. H. Hong, M. O. Shneier, G. Holguin, J. Park, R. Eastman, "Dynamic 6DOF Metrology for Evaluating a Visual Servoing" Proceedings of the 2008 Performance Metrics for Intelligent Systems (PerMIS) Workshop, Gaithersburg, MD, United States, 08/19/2008 to 08/21/2008, pp. 173-180, (21-Aug-2008)

\footnotetext{
Legend:

F - NIST 114 Filed

S - Submitted to ERB

A - ERB Approved

$\mathbf{R}$ - Refereed

I - Invited
} 
J. H. Daruwalla, N. G. Dagalakis, "Calibration of a Force Feedback Joystick" Calibration of a Force Feedback Joystick, , (29Aug-2008)

S. Condon, J. Phillips, C. Doran, J. Aberdeen, D. Parvaz, B. Oshika, G. A. Sanders, C. I. Schlenoff, "Applying Automated Metrics to Speech Translation Dialogs" Proceedings of the 2008 LREC (Language Resources and Evaluation Conference), Morocco, -1, United States, 05/28/2008 to 05/30/2008, (30-Jun-2008)

C. J. Scrapper Jr, R. Madhavan, S. B. Balakirsky, "Performance Analysis for Stable Mobile Robot Navigation Solutions" Proceedings of the SPIE Defense and Security Symposium, SPIE Defense and Security Symposium 2008, Orlando, FL, United States, 03/18/2008 to 03/20/2008, (20-Mar-2008)

N. G. Dagalakis, Y.S. Kim, D. S. Sawyer, C. M. Shakarji, "Development of Tools for Measuring the Performance of Computer Assisted Orthopaedic Hip Surgery Systems" Proceedings of the Performance Metrics for Intelligent Systems | 2007 | PerMIS |, Performance Metrics for Intelligent Systems Workshop (PerMIS) 2007, Gaithersburg, MD, 08/29/2007 to 08/30/2007, (28-Dec2007)

B. K. Taylor, S. B. Balakirsky, E. Messina, R. D. Quinn, "Design and Validation of a Whegs Robot in USARSim" Proceedings of PerMIS | 2007 |, Performance Metrics for Intelligent Systems (PerMIS) 2007, Gaithersburg, MD, 08/28/2007 to 08/30/2007, (31-Jan-2008)

H.M. Huang, E. Messina, "Autonomy Levels for Unmanned Systems (ALFUS) FrameworkVolume II: Framework Models Initial Version" Special Publication (NIST SP) 1011-II-1.0, (28-Dec-2007)

J. S. Albus, R. V. Bostelman, T. H. Hong, T. Chang, W. P. Shackleford, M. O. Shneier, "Integrating Learning Into a Hierarchical Vehicle Control System" ICAE Journal, (31-Dec-2007)

C. J. Scrapper Jr, R. Madhavan, S. B. Balakirsky, "Using a High-Fidelity Simulation Framework for Performance Singularity Identification and Testing" Proceedings of Applied Imagery Pattern Recognition (AIPR) 2007, Washington, DC, United States, 10/10/2007 to 10/12/2007, (21-Nov-2007)

F. Johnson, "Exploitation of Security Vulnerabilities Inherent in Common Development of Process Control Systems" Poster Session, 17th USENIX Security Symposium (USENIX Security 08), 07/28/2008 to 08/01/2008, San Jose, CA, -, United States, (01-Aug-2008)

J. C. Chiao, J. M. Goldman, D. A. Heck, P. Kazanzides, W. J. Peine, J. B. Stiehl, D. Yen, N. G. Dagalakis, "Metrology and Standards Needs of Some Categories of Medical Devices" Journal of Research (NIST JRES), (30-Apr-2008)

\footnotetext{
Legend:

F - NIST 114 Filed

S - Submitted to ERB

A - ERB Approved

$\mathbf{R}$ - Refereed

I - Invited
} 


\title{
Manufacturing Systems Integration Division
}

\author{
List of Publications
}

For

FY2008 to FY2010

Publication Type - All Publication Type

(October 1, 2007 to March 1, 2010) 
FY2010 Publications:

G. Ameta, M. Mani, R. Sudarsan, K. W. Lyons, S. C. Feng, R. D. Sriram, "Carbon Weight Analysis for Machining Operation and Allocation for Redesign" NIST Interagency/Internal Report (NISTIR) 7560, 23 pp., (16-Dec-2009)

G. Shao, N. E. Bengtsson, B. J. Johansson, "Interoperability for Simulation of Sustainable Manufacturing" Proceedings of the 2010 Spring Simulation Multi-conference (SpringSim '10), Orlando, FL, United States, 04/12/2010 to 04/16/2010, 10 pp., (24Feb-2010)

D. Kibira, G. Shao, S. -. Nowak, "System Dynamics Modeling of Corn Ethanol as a Bio Transportation Fuel in the United States" NIST Interagency/Internal Report (NISTIR) 7649, 22 pp., (28-Jan-2010)

J. Cuvillier, K. C. Morris, "QOD Standalone Users Guide" NIST Interagency/Internal Report (NISTIR) 7662, 15 pp., (16-Dec2009)

S. Fenves, E. Subrahmanian, M. Mani, A. T. Jones, "An Enabler for Supplier Discovery in Virtual Supply Chains: A Shared Terminology" NIST Interagency/Internal Report (NISTIR) 7647, 10 pp., (05-Nov-2009)

B. J. Johansson, A. Skoogh, M. Mani, S. K. Leong, "Discrete Event Simulation as Requirements Specification for Sustainable Manufacturing Systems Design" Proceedings of the PerMIS'09 Conference, Gaithersburg, MD, United States, 09/21/2009 to 10/23/2009, 12 pp., (04-Dec-2009)

S. C. Feng, H. Lee, C. B. Joung, P. Ghodous, "Development Overview of an Information Model for Disassembly" Proceedings of the 7th Global Conference on Sustainable Manufacturing, Chennai, -1, India, 12/02/2009 to 12/04/2009, 7 pp., (23-Dec2009)

S. K. Leong, G. Shao, N. E. Bengtsson, "THE ROLE OF SIMULATION IN TRACKING MOBILE ASSETS USING AUTOMATIC IDENTIFICATION SYSTEMS" Proceedings of the National Defense Industrial Association (NDIA) 12th Annual Systems Engineering Conference, San Diego, CA, United States, 10/26/2009 to 10/29/2009, (15-Oct-2009)

D. Kibira, S. K. Leong, "TEST OF CORE MANUFACTURING SIMULATION DATA SPECIFICATION IN AUTOMOTIVE ASSEMBLY" Proceedings of the Simulation Interoperability Standards Organization (SISO)and Society for Modeling and Simulation (SCS) International European Multi Conference , Orlando, FL, United States, 04/12/2010 to 04/16/2010, 10 pp., (19-Jan-2010)

(R) F. Neuhaus, "The Semantics of Modules in Common Logic" Interontology 2010, Tokyo, -1, Japan, 02/27/2010 to 02/28/2010, 5 pp., (20-Jan-2010)

S. C. Feng, C. B. Joung, "An Overview of a Proposed Measurement Infrastructure for Sustainable Manufacturing" Proceedings of the 7th Global Conference on Sustainable Manufacturing, Chennai, China, 12/02/2009 to 12/04/2009, 12 pp., (23-Dec-2009)

C. Bock, X. Zha, H.W. Suh, J.H. Lee, "Ontological Product Modeling for Collaborative Design" NIST Interagency/Internal Report (NISTIR) 7643, (30-Oct-2009)

\footnotetext{
Legend:

F - NIST 114 Filed

S - Submitted to ERB

A - ERB Approved

$\mathbf{R}$ - Refereed

I - Invited
} 
R. D. Sriram, A. T. Jones, M. Mani, R. Sudarsan, E. Subrahmanian, "NETWORK-CENTRIC PRODUCT REALIZATION AND THE ROLE OF STANDARDS" International Conference on Frontiers of Interface between Statistics and Sciences, Hyderabad, -1, India, 12/30/2009 to 01/02/2010, 6 pp., (18-Feb-2010)

S. C. Feng, C. B. Joung, "Development Overview of Sustainable Manufacturing Metrics" Proceedings of the 17th CIRP International Conference on Life Cycle Engineering 2010, Anhui, -1, China, 05/19/2010 to 05/21/2010, 6 pp., (18-Feb-2010)

\title{
FY2009 Publications:
}

A. Cardone, R. D. Sriram, W. Albers, H. C. Pant, "Study of interaction of the cyclin-dependent kinase 5 with its activator, p25 and with the p25-derived inhibitor, CIP"NIST Interagency/Internal Report (NISTIR) 7552, (15-Jan-2009)

M. Mani, A. T. Jones, J.H. Shin, R. D. Sriram, "Towards Information Networks to Support Composable Manufacturing" Proceedings of the PERMIS Workshop, Gaithersburg, MD, United States, 08/19/2008 to 08/21/2008, (15-Oct-2008)

M. Vujasinovic, N. Ivezic, B. Kulvatunyou, E. J. Barkmeyer, M. Missikoff, F. Taglino, Z. Marjanovic, I. Miletic, "A SemanticMediation Architecture for Interoperable Supply-Chain Applications" International Journal of Computer Integrated Manufacturing, 15 pp., (10-Nov-2008)

S. Krima, R. Barbau, X. Fiorentini, R. Sudarsan, R. D. Sriram, "OntoSTEP: OWL-DL Ontology for STEP" NIST Interagency/Internal Report (NISTIR) 7561, (04-May-2009)

X. Fiorentini, R. Sudarsan, H.W. Suh, J.H. Lee, R. D. Sriram, "An Evaluation of Description Logic for the Development of Product Models" Journal of Computing and Information Science in Engineering, 20 pp., (10-Nov-2008)

M. Sarigecili, R. Uptal, R. Sudarsan, R. D. Sriram, "Development of Functionality-Based Conformance Classes for ISO 10303 Conformance Classes for Tolerancing Functionality" Proceedings of the CAD09 Conference, Reno, NV, United States, 06/08/2009 to 06/12/2009, (15-Jun-2009)

S. J. Kemmerer, "Manufacturing Interoperability Program, a Synopsis" NIST Interagency/Internal Report (NISTIR) 7533, (24Feb-2009)

P. O. Denno, E. J. Barkmeyer, F. Neuhaus, "Use of Semantic Mediation in Manufacturing Supply Chains"Book Title: Use of Semantic Mediation in Manufacturing Supply Chains, IGI Global, 701 E. Chocolate Avenue, Suite 200, Hershey, PA, 17033, United States, (06-Apr-2009)

F. Neuhaus, "Ontology-based Technologies - Technology Transfer from Bioinformatics?" Proceedings of the Ontology for the Intelligence Community 2008, Columbia, MD, United States, 11/30/2008 to 12/01/2008, 3 pp., (05-Nov-2008)

(R) M. Vujasinovic, N. Ivezic, B. Kulvatunyou, E. J. Barkmeyer, M. Missikoff, F. Taglino, Z. Marjanovic, I. Miletic, "SemanticMediation for Standards-based B2B Interoperability" IEEE Internet Computing, (15-Jun-2009)

S. Jain, "Tradeoffs in Building a Generic Supply Chain Simulation Capability" 2008 Winter Simulation Conference, Miami,

\author{
Legend: \\ F - NIST 114 Filed \\ S - Submitted to ERB \\ A - ERB Approved \\ R - Refereed \\ I - Invited
}


FL, United States, 12/07/2008 to 12/10/2008, (15-Oct-2008)

J. Lubell, B. Harvey, P. Goyal, K. C. Morris, "NDRProfile Schema Version 1.0 User Guide" NIST Interagency/Internal Report (NISTIR) 7547, 14 pp., (16-Dec-2008)

G. Shao, S. K. Leong, C. R. McLean, "Simulation-based Manufacturing Interoperability Standards and Testing" Proceedings of the 9th International Conference on Progress of Machining Technology, Kunming, China, 04/25/2009 to 04/28/2009, (07-Jan2009)

K. C. Morris, S. P. Frechette, P. Goyal, J. Lubell, B. Kulvatunyou, S. Sidi Malick, N. Brayard, S. Tixier, "User's Guide for the Quality of Design Testing Tool and the Content Checker"NIST Interagency/Internal Report (NISTIR) 7538, 35 pp., (05-Nov2008)

P. Sarkar, R. Sudarsan, H.W. Suh, K. W. Lyons, R. D. Sriram, "A Measure of Product Sustainability Based on Triple Bottom Line" Proceedings of the ASME 2009 International Design Engineering Technical Conferences \& Computers and Information in Engineering Conference IDETC/CIE 2009

, same, San Diego, CA, United States, 08/30/2009 to 09/02/2009, (15-Jun-2009)

N. Krdzavac, C. Bock, "Reasoning in Manufacturing Part-Part Examples with OWL 2" NIST Interagency/Internal Report (NISTIR) 7535, 21 pp., (29-Oct-2008)

(RI) J. Lubell, B. Kassel, R. Sudarsan, "Descriptive Metadata Requirements for Long-term Archival of Digital Product Models" Proceedings of the Indo-US Workshop on International Trends in Digital Preservation, Indo-US Workshop on International Trends in Digital Preservation, Pune, -1, India, 03/24/2009 to 03/25/2009, (25-Feb-2009)

(R) J. Lubell, "Documenting and Implementing Guidelines with Schematron" Proceedings of Balisage: The Markup Conference 2009, Balisage Series on Markup Technologies. doi:10.4242/BalisageVol3.Lubell01, Montreal, -1, Canada, 08/11/2009 to 08/14/2009, Vol. 3, (26-Aug-2009)

G. Shao, C. R. McLean, A. Brodsky, P. Ammann, "PARAMETER VALIDATION USING CONSTRAINT OPTIMIZATION FOR MODELING AND SIMULATION" Proceedings of the Industrial Simulation Conference 2009, Loughborough, -1, United Kingdom, 06/01/2009 to 06/03/2009, (11-May-2009)

J.C. Cheng, K. H. Law, A. T. Jones, R. D. Sriram, "Service Oriented and Orchestrated Framework for Supply Chain Integration" Proceedings of the ASME 2009 International Design Engineering Technical Conferences \& Computers and Information in Engineering Conference IDETC/CIE 2009

, San Diego, CA, United States, 08/30/2009 to 09/02/2009, (08-Jun-2009)

(R) M. Vujasinovic, E. J. Barkmeyer, N. Ivezic, Z. Marjanovic, "Interoperable Supply-Chain Applications: Message Metamodelbased Semantic Reconciliation of B2B Messages" International Journal of Cooperative Information Systems, (13-May-2009)

M. S. Lanz, R. Tuokko, "GENERIC REFERENCE ARCHITECTURE FOR DIGITAL, VIRTUAL, AND REAL

\footnotetext{
Legend:

F - NIST 114 Filed

S - Submitted to ERB

A - ERB Approved

R - Refereed

I - Invited
} 
REPRESENTATIONS OF MANUFACTURING SYSTEMS" Proceedings of the Indo-US Workshop on Designing Sustainable Products, Services, and Manufacturing Systems, Bangalore, India, 08/18/2009 to 08/20/2009, (13-Aug-2009)

K. W. Lyons, M. T. Postek, "A Decade of Commitment from the NIST Manufacturing Engineering Laboratory to Nanomanufacturing and Nanometrology" Proceedings of the SPIE Optics and Photonics NanoScience and Engineering Conference 2009, San Diego, CA, United States, 08/02/2009 to 08/06/2009, (27-Aug-2009)

K. C. Morris, J. Lubell, S. Sidi Malick, "Framework for XML Schema Naming and Design Rules Development Tools" NIST Interagency/Internal Report (NISTIR) 7589, (09-Jul-2009)

S. Jain, C. McLean, "Recommended Practices for Homeland Security Modeling and Simulation" Proceedings of the 2009 Winter Simulation Conference, Austin, TX, United States, 12/13/2009 to 12/16/2009, (13-Aug-2009)

A. Weissman, S. K. Gupta, X. Fiorentini, R. Sudarsan, R. D. Sriram, "Formal Representation of Product Design Specifications for Validating Product Designs" NIST Interagency/Internal Report (NISTIR) 7626, (27-Aug-2009)

N. E. Bengtsson, G. Shao, Y.T. T. Lee, S. K. Leong, C. R. McLean, B. Johansson, A. Skoogh, "Input Data Management Methodology for Discrete Event Simulation" Proceedings of the 2009 Winter Simulation Conference, Austin, TX, United States, $12 / 13 / 2009$ to $12 / 16 / 2009$, (26-Aug-2009)

D. Kibira, S. Jain, C. McLean, "A System Dynamics Modeling Framework for Sustainable Manufacturing" Proceedings of the 27th Annual System Dynamics Society Conference, Albuquerque, NM, United States, 07/26/2009 to 07/30/2009, (10-Aug2009)

X. Fiorentini, R. Sudarsan, S.R. Ray, R. D. Sriram, "Towards a Method for Harmonizing Information Standards" Proceedings of the fifth annual IEEE Conference on Automation Science and Engineering (IEEE CASE 2009), Bangalore, -1, India, 08/22/2009 to 08/25/2009, (19-Aug-2009)

S. Krima, R. Barbau, X. Fiorentini, R. Sudarsan, S. Foufou, R. D. Sriram, "OntoSTEP: OWL-DL Ontology for STEP" Proceedings of the 6th International Conference on Product Lifecycle Management, Bath, -1, United Kingdom, 07/06/2009 to 07/08/2009, (15-Jun-2009)

C. R. McLean, S. Jain, Y.T. T. Lee, "Overview of MSA Needs for Homeland Security" Proceedings of the Interservice/Industry Training, Simulation \& Education Conference (iitsec.org), Orlando, FL, United States, 11/30/2009 to 12/03/2009, (29-Jul-2009)

(R) S. C. Feng, C. B. Joung, T. V. Vorburger, "Characterization of Probe Dynamic Behaviors in Critical Dimension Atomic Force Microscopy" International Journal of Nanotechnology, (15-Oct-2008)

(I) R. D. Sriram, S. Fenves, "The Role for Engineers in Health Care Information Systems" Industrial Engineer, (30-Dec-2008) B. J. Johansson, A. Fasth, J. Stahre, J. Heilala, S. K. Leong, Y.T. T. Lee, F. H. Riddick, "Enabling Flexible Manufacturing Systems by Using Level of Automation as Design Parameter" Proceedings of the 2009 Winter Simulation Conference, Austin, TX, United States, 12/13/2009 to 12/16/2009, (19-Aug-2009)

\footnotetext{
Legend:

F - NIST 114 Filed

S - Submitted to ERB

A - ERB Approved

R - Refereed

I - Invited
} 
(I) R. Sudarsan, R. D. Sriram, P. Sarkar, "Metrics, Standards and Industry Best Practices for Sustainable Manufacturing Systems" The fifth annual IEEE Conference on Automation Science and Engineering (IEEE CASE 2009), , Bangalore, -1, India, 08/22/2009 to 08/25/2009, (10-Aug-2009)

G. Shao, "System Testing Using Use Cases for an ER Simulation Model" Proceedings of the North-American Simulation Technology Conference (NASTEC 09), Atlanta, GA, United States, 08/26/2009 to 08/28/2009, (29-Jul-2009)

F. H. Riddick, Y.T. T. Lee, "Representing Layout Information in the CMSD Specification" Proceedings of the 2009 Fall Simulation Interoperability Workshop of the Simulation Interoperability Standards Organization (SISO), Orlando, FL, United States, 09/21/2009 to 09/25/2009, (26-Aug-2009)

X. Fiorentini, R. Sudarsan, "STEP-OAGIS Harmonization Joint Working Group, PDM Subgroup Interim Report" NIST Interagency/Internal Report (NISTIR) 7618, (27-Aug-2009)

\section{FY2008 Publications:}

S. C. Feng, C. B. Joung, T. V. Vorburger, "A Characterization of Probe Dynamic Behaviors in Critical Dimension Atomic Force Microscopy" Scanning 2008, Gaithersburg, MD, 04/15/2008 to 04/17/2008, 2 pp., (17-Mar-2008)

X. Fiorentini, R. Sudarsan, M. Mani, S. Fenves, R. D. Sriram, "An Evaluation of Description Logic for the Development of Product Models" NIST Interagency/Internal Report (NISTIR) 7481, 31 pp., (15-Apr-2008)

R. D. Sriram, S. Fenves, "Manufacturing Metrology and Standards for the Health Care Enterprise Program Summary "NIST Interagency/Internal Report (NISTIR) 7487, 10 pp., (07-Apr-2008)

J. Kim, B. Kulvatunyou, N. Ivezic, A. T. Jones, "A Layered Approach to Semantic Similarity Analysis of XML Schemas" Proceedings of the 2008 IEEE International Conference on Information Reuse and Integration, Las Vegas, NV, 07/13/2008 to 07/15/2008, 8 pp., (07-Apr-2008)

R. Sudarsan, E. Subrahmanian, "Engineering Informatics: Introduction to the Special Issue" Journal of Computing and Information Science in Engineering, (01-Mar-2008)

(I) R. Gao, R. Yan, L. Zhang, K. B. Lee, "Condition Monitoring of Operating Spindle Based on Stochastic Subspace Identification" 2007 ASME International Mechanical Engineering Congress and Exposition, 2007 ASME International Mechanical Engineering Congress and Exposition, Seattle, WA, 11/11/2007 to 11/15/2007, pp. 1-7, (11-Nov-2007)

S. C. Feng, S. P. Frechette, "The State of Container Security Standards" NIST Interagency/Internal Report (NISTIR) 7153, (17Oct-2007)

P. O. Denno, T. Thurman, J. Mettenburg, D. Hardy, "On Enabling a Model-based Systems Engineering Discipline" The 18th International Symposium of the International Council on Systems Engineering (INCOSE), The Netherlands, Netherlands, 06/15/2008 to 06/19/2008, 10 pp., (28-May-2008)

\footnotetext{
Legend:

F - NIST 114 Filed

S - Submitted to ERB

A - ERB Approved

R - Refereed

I - Invited
} 
R. D. Sriram, A. S. Deshmukh, A. G. Banerjee, S. K. Gupta, "Content-Based Assembly Search: A Step Towards Assembly Reuse" To Appear in the Journal of CAD, (07-Nov-2007)

G. Ameta, R. Sudarsan, X. Fiorentini, M. Mani, S. Fenves, K. W. Lyons, R. D. Sriram, "Extending the Notion of Quality from Physical Metrology to Information and Sustainability" NIST Interagency/Internal Report (NISTIR) 7517, 34 pp., (30-Jul-2008)

J. Lubell, M. Mani, E. Subrahmanian, R. Sudarsan, "Long Term Sustainment Workshop Report" NIST Interagency/Internal Report (NISTIR) 7496, 24 pp., (26-Mar-2008)

D. Kibira, C. R. McLean, "Modeling and Simulation for Sustainable Manufacturing" Proceedings of the 2nd IASTED 2008 Africa Conference on Modeling and Simulation, SCIENCE AND TECHNOLOGY INNOVATION FOR SUSTAINABLE DEVELOPMENT, Gaborone, Botswana, 09/08/2008 to 09/10/2008, 11 pp., (11-Jun-2008)

J. Lubell, R. Sudarsan, E. Subrahmanian, M. Mani, "Sustaining Engineering Informatics: Toward Methods and Metrics for Digital Curation" International Journal of Digital Curation, Vol. 3, No. 2, 11 pp., (01-Jul-2008)

S. K. Leong, M. Johansson, B. Johansson, Y.T. T. Lee, F. H. Riddick, "A Real World Pilot implementation of the Core Manufacturing Simulation Information Model" Proceedings of the Simulation Interoperability Standards Organization (SISO) Spring 2008 SIW Workshop, 2008 Euro Simulation Interoperability Workshop, Edinburgh, Scotland, United States, 06/16/2008 to $06 / 19 / 2008,11$ pp., (23-Jun-2008)

G. Shao, C. R. McLean, "ER Simulation Prototypes for Incident Management Training" Industrial Simulation Conference 2008 , Lyon, France, 06/09/2008 to 06/11/2008, 10 pp., (19-May-2008)

M. Mani, K. W. Lyons, R. Sudarsan, E. Subrahmanian, R. D. Sriram, "Introducing Sustainability Early into Manufacturing Process Planning" Proceedings of the 14th International Conference on Manufacturing Science and Engineering, ASME, Evanston, IL, 10/07/2008 to 10/10/2008, 7 pp., (07-May-2008)

(R) S. Jain, C. R. McLean, "Components for an Incident Management Simulation and Gaming Framework and Related Developments " Simulation-Transactions of the Society for Modeling and Simulation International, 52 pp., (12-Mar-2008)

(R) G. Shao, Y.T. T. Lee, "Applying Software Product Line Technology to Simulation Modeling of Emergency Response Facility" Simulation-Transactions of the Society for Modeling and Simulation International, (15-Jul-2008)

A. Bouras, R. Sudarsan, E. Subrahmanian, J.P. Legrange, "ICT for Supply Chains and Product Lifecycle Management: A Research Agenda for French-US Collaboration" NIST Interagency/Internal Report (NISTIR), (24-Oct-2007)

C. R. McLean, S. Jain, Y.T. T. Lee, "A Taxonomy of Homeland Security Modeling, Simulation, and Analysis Applications " Proceedings of the Simulation Interoperability Standards Organization (SISO) Spring 2008 SIW Workshop, 2008 SISO SIW Workshop, Providence, RI, 04/14/2008 to 04/18/2008, 12 pp., (14-Apr-2008)

S. Kim, S.Y. Shin, I.H. Lee, S.J. Kim, R. D. Sriram, B.T. Zhang, "PIE: An Online Prediction System for Protein-Protein Interactions from Text" Nucleic Acid Research, Special Issue on Web Services, 12 pp., (17-Apr-2008)

\footnotetext{
Legend:

F - NIST 114 Filed

S - Submitted to ERB

A - ERB Approved

R - Refereed

I - Invited
} 
C. R. McLean, S. Jain, Y.T. T. Lee, "Homeland Security Simulation Domain: A Needs Analysis Overview" Simulation Interoperability Standards Organization (SISO) 2008 European Simulation Interoperability Workshop (2008 Euro SIW), Edinburgh, United States, 06/16/2008 to 06/19/2008, 9 pp., (19-May-2008)

Y.T. T. Lee, F. H. Riddick, C. R. McLean, S. K. Leong, "Current Activities Related to the Core Manufacturing Simulation Data Standards Development Effort" Proceedings of the Fall 2008 Simulation Interoperability Workshop, Orlando, FL, United States, 09/14/2008 to 09/19/2008, 11 pp., (07-Aug-2008)

V. Liang, C. Bock, X. Zha, "An Ontological Modeling Platform" NIST Interagency/Internal Report (NISTIR) 7509, 20 pp., (04-Jun-2008)

X. Zha, R. D. Sriram, M. Fernandez, F. Mistree, "Knowledge-Intensive Collaborative Decision Support for Design Processes: A Hybrid Decision Support Model and Agent" Computers in Industry, 15 pp., (10-Sep-2008)

F. H. Riddick, Y.T. T. Lee, "REPRESENTING LAYOUT INFORMATION IN THE CMSD SPECIFICATION" 2008 Winter Simulation Conference, Miami, FL, United States, 12/07/2008 to 12/10/2008, 11 pp., (09-Jul-2008)

K. C. Morris, P. Goyal, S. P. Frechette, "Development Life Cycle and Tools for Data Exchange Specification" Proceedings of the ASME 2008 International Design Engineering Technical Conferences \& Computers and Information in Engineering Conference, IDETC/CIE 2008, Brooklyn, NY, United States, 08/03/2008 to 08/06/2008, 12 pp., (19-May-2008)

G. Roberts, S. B. Balakirsky, S. Foufou, "3D Reconstruction of Rough Terrain for USARSim Using a Height-map Method" Proceedings of the 2008 Performance Metrics for Intelligent Systems (PerMIS) Workshop, Gaithersburg, MD, United States, 08/19/2008 to 08/21/2008, pp. 259-264, (21-Aug-2008)

C. P. Cheng, J. Pan, G. T. Lau, K. H. Law, A. T. Jones, "Relating Taxonomies with Regulations" Proceedings of the 9th Annual International Conference on Digital Government Research, Montreal, Canada, 05/18/2008 to 05/21/2008, 14 pp., (13-May2008)

P. Hoffmann, S. C. Feng, G. Ameta, P. Ghodous, L. Qiao, "Towards a Multi-View Semantic Model for Product Feature Description" Concurrent Engineering 2008, Belfast, 07/24/2008 to 07/25/2008, 7 pp., (28-May-2008)

T. V. Vorburger, R. G. Dixson, J. N. Fu, N. Orji, S. C. Feng, M. W. Cresswell, R. A. Allen, W. F. Guthrie, W. Chu, "Nano- and Atomic-Scale Length Metrology" Proc. 5th Int. Conf. on Precision, Meso, Micro, and Nano Engineering (COPEN 2007) (Allied Publishers Pvt. Ltd, Chennai, 2007), 5th Int. Conf. on Precision, Meso, Micro, and Nano Engineering (COPEN 2007), Trivandrum, India, 12/14/2007 to 12/14/2007, pp. 13-18, (14-Dec-2007)

K. W. Lyons, M. T. Postek, "Metrology at the Nanoscale: What are the Grand Challenges?" SPIE NanoScience and Engineering, San Diego, CA, United States, 08/10/2008 to 08/14/2008, 12 pp., (06-Aug-2008)

\footnotetext{
Legend:

F - NIST 114 Filed

S - Submitted to ERB

A - ERB Approved

$\mathbf{R}$ - Refereed

I - Invited
} 
